\title{
Supramolecular Bacterial Systems
}

Shrilerishnan Sankaran

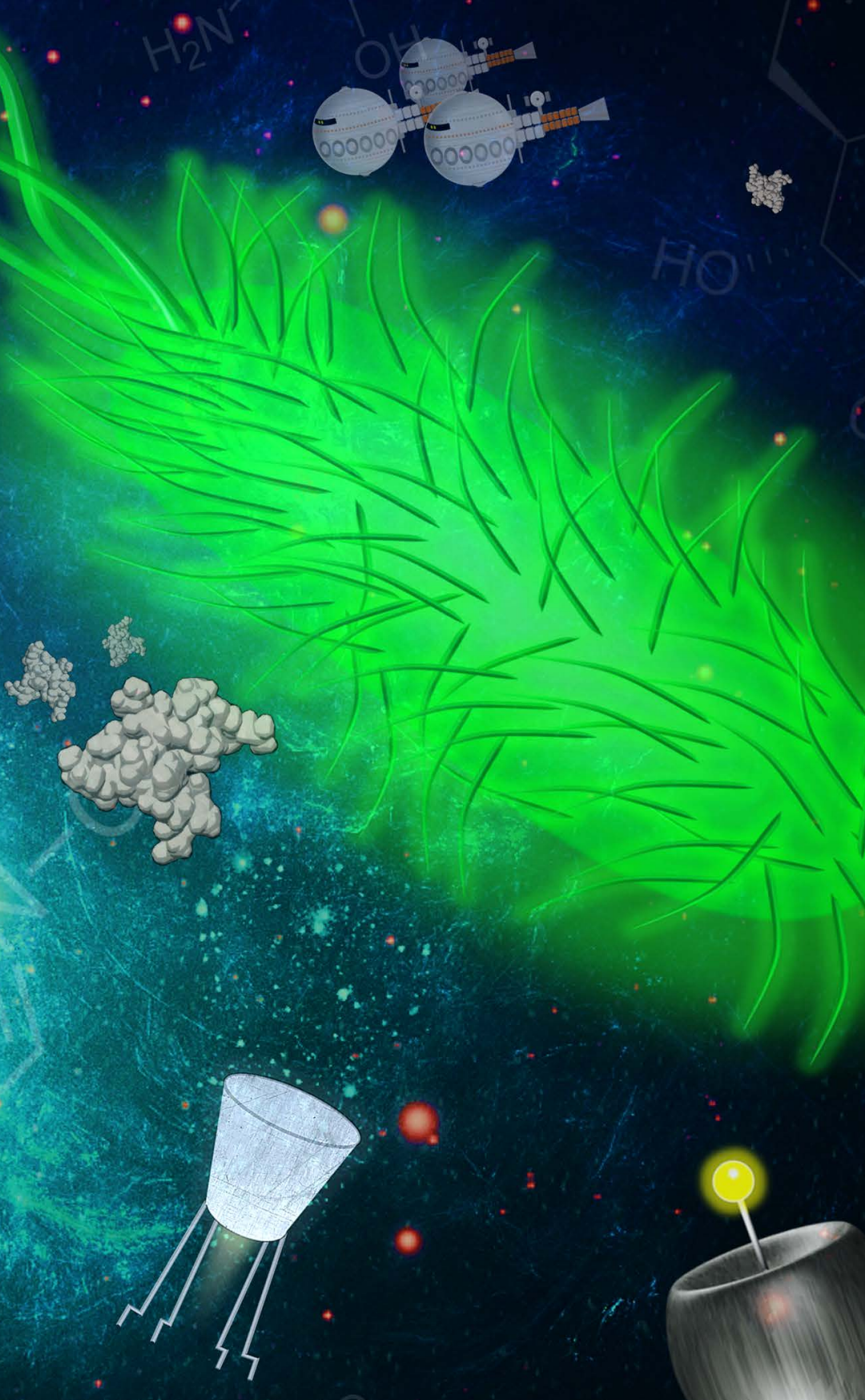




\section{Supramolecular Bacterial Systems}

Shrikrishnan Sankaran 


\section{Members of the committee:}

\begin{tabular}{|c|c|c|}
\hline Chairman: & Prof. dr. ir. J.W.M. Hilgenkamp & (University of Twente) \\
\hline Promotor: & Prof. dr. ir. P. Jonkheijm & (University of Twente) \\
\hline \multirow[t]{5}{*}{ Members: } & Prof. dr. ir. L. Brunsveld & $\begin{array}{l}\text { (Eindhoven University } \\
\text { of Technology) }\end{array}$ \\
\hline & FTOI. U. II. IVIVIV.A.E. CIdessenIS & (umiversicy or iwemie) \\
\hline & Prof. dr. ir. J. Huskens & (University of Twente) \\
\hline & Dr. ir. Séverine le Gac & (University of Twente) \\
\hline & Prof. dr. A.H. Velders & (Wageningen University) \\
\hline
\end{tabular}

The research described in this thesis was performed within the laboratories of the Bioinspired Molecular Engineering Laboratory (BMEL), MIRA Institute for Biomedical Technology and Technical Medicine and the Molecular Nanofabrication (MnF) group, MESA+ institute for Nanotechnology, Department of Science and Technology (TNW) of the University of Twente. This research was supported by the European Research Council through Starting Grant Sumoman (259183).

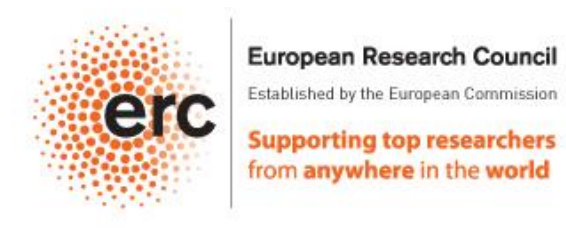

\section{Supramolecular Bacterial Systems}

Copyright $\odot$ 2015, Shrikrishnan Sankaran, Enschede, The Netherlands.

All rights reserved. No part of this thesis may be reproduced or transmitted in any form, by any means, electronic or mechanical without prior written permission of the author.

ISBN: $\quad 978-90-365-3996-8$

DOI: $\quad 10.3990 / 1.9789036539968$

Cover art: Jenny Brinkmann-Sankaran

Printed by: Gildeprint Drukkerijen - The Netherlands 


\title{
Supramolecular Bacterial Systems
}

\author{
DISSERTATION
}

to obtain

the degree of doctor at the University of Twente,

on the authority of the rector magnificus

Prof. dr. H. Brinksma,

on account of the decision of the graduation committee, to be publicly defended on Wednesday December 16, 2015 at 14.45 h

by

Shrikrishnan Sankaran

Born on January 7, 1988

in Bangalore, India 
This dissertation has been approved by:

Promotor: Prof. dr. ir. P. Jonkheijm 
"We shall not cease from exploration, and the end of all our exploring will be to arrive where we started and know the place for the first time." 



\section{Table of Contents}

\begin{tabular}{|c|c|c|c|}
\hline \multicolumn{2}{|c|}{ Chapter 1: } & The supramolecular bacterial systems toolbox & \multirow{2}{*}{$\frac{1}{2}$} \\
\hline 1.1. & Intro & uction & \\
\hline \multirow[t]{4}{*}{1.2.} & \multicolumn{2}{|c|}{ Bacteria } & 3 \\
\hline & 1.2.1. & Bacterial Adhesion & 3 \\
\hline & 1.2.2. & Recombinant DNA technology & 5 \\
\hline & 1.2.3. & $\begin{array}{l}\text { Bacteria in nanotechnology and biomedical } \\
\text { engineering }\end{array}$ & 7 \\
\hline \multirow[t]{3}{*}{1.3.} & \multicolumn{2}{|c|}{ Proteins } & 10 \\
\hline & 1.3.1. & Fluorescent proteins & 10 \\
\hline & 1.3.2. & Cystine-stabilized miniproteins & 12 \\
\hline \multirow[t]{4}{*}{1.4} & \multicolumn{2}{|c|}{ Supramolecular host-guest chemistry } & 15 \\
\hline & 1.4.1. & Hosts & 15 \\
\hline & 1.4 .2 & Guests & 18 \\
\hline & $1.4 \cdot 3$ & Stimuli Responsiveness & 22 \\
\hline 1.5 . & \multicolumn{2}{|c|}{ Outline of thesis } & 23 \\
\hline 1.6. & \multicolumn{2}{|c|}{ References } & 24 \\
\hline
\end{tabular}

\begin{tabular}{|c|c|c|}
\hline Chapter 2: & $\begin{array}{l}\text { Multivalent peptide tag for supramolecular } \\
\text { protein immobilization on } \beta \text {-cyclodextrin modified } \\
\text { surfaces }\end{array}$ & 31 \\
\hline 2.1. & Introduction & 32 \\
\hline \multirow[t]{6}{*}{ 2.2. } & Results and Discussions & 34 \\
\hline & Peptide tag & 34 \\
\hline & Recombinant protein & 36 \\
\hline & SPR analysis & 37 \\
\hline & Continuous flow microspotting & 38 \\
\hline & 2.2.5. $\quad \beta$-CD silica nanoparticles & 41 \\
\hline 2.3. & Conclusions & 42 \\
\hline
\end{tabular}


2.4. Acknowledgements

2.5. Experimental Section 43

\begin{tabular}{ll} 
2.6. & References \\
\hline
\end{tabular}

\section{Chapter 3: $\quad$ Supramolecular surface immobilization of knottin 51 derivatives for dynamic display of high affinity binders}

\begin{tabular}{|c|c|c|c|}
\hline \multirow{2}{*}{$\begin{array}{l}3.1 . \\
3.2 .\end{array}$} & \multicolumn{2}{|c|}{ Introduction } & 52 \\
\hline & \multicolumn{2}{|c|}{ Results and Discussions } & 55 \\
\hline & 3.2.1. & Design and Recombinant Synthesis of Knottins & 55 \\
\hline & 3.2.2. & $\begin{array}{l}\text { Evaluating Trypsin Inhibitory Functionality of } \\
\text { Knottin Constructs }\end{array}$ & 57 \\
\hline & 3.2.3. & $\begin{array}{l}\text { Evaluating } \mathrm{CB}[8] \text {-binding Capability of Knottin } \\
\text { Constructs }\end{array}$ & 59 \\
\hline & 3.2.4. & Supramolecular Surface Immobilization of Knottins & 60 \\
\hline 3.3. & \multicolumn{2}{|c|}{ Conclusions } & 64 \\
\hline 3.4. & \multicolumn{2}{|c|}{ Acknowledgements } & 65 \\
\hline 3.5 . & \multicolumn{2}{|c|}{ Experimental Section } & 65 \\
\hline 3.6. & \multicolumn{2}{|c|}{ References } & 72 \\
\hline 3.7. & \multicolumn{2}{|c|}{ Supporting information } & 75 \\
\hline
\end{tabular}

\begin{tabular}{lcc}
\hline Chapter 4: & Scaffolding of cystine-stabilized miniproteins & 77 \\
\hline 4.1. $\quad$ Introduction & 78
\end{tabular}

4.2. Results and Discussions $\quad 82$

4.2.1. Design and recombinant synthesis strategy 82

4.2.2. Trypsin inhibition analysis 83

4.2.3. MS analysis of protein binding to miniprotein 85 chains

4.2.4. MST analysis of protein binding to miniprotein 88 chains

4.2.5. SPR analysis of protein binding to miniprotein 89 chains 
4.3. Conclusions $\quad 92$

4.4. Acknowledgements 93

4.5. Experimental Section 93

$\begin{array}{ll}\text { 4.6. References } & 104\end{array}$

Chapter 5: Optical control over bioactive ligands at 107 supramolecular surfaces

\begin{tabular}{lll}
\hline 5.1. & Introduction & 108 \\
5.2. & Results and Discussions & 110 \\
& 5.2.1. QCM Characterization & 110 \\
& 5.2.2. Microcontact printing & 112 \\
& 5.2.3. Photo-responsiveness & 114 \\
5.3. & Conclusions & 115 \\
5.4. & Acknowledgements & 116 \\
5.5. & Experimental Section & 116 \\
5.6. & References & 127 \\
5.7. & Supporting Information & 130 \\
\hline
\end{tabular}

Chapter 6: Photo-responsive cucurbit[8]uril-mediated adhesion of bacteria on supported lipid bilayers

$\begin{array}{lll}6.1 . & 132\end{array}$

6.2. Results and Discussions 135

6.2.1. Selection of a non-fouling MV2+-functionalized 135 layer

6.2.2. Incorporating supramolecular components $\quad 139$

6.2.3. Bacterial capture and release 143

$\begin{array}{ll}\text { 6.3. Conclusions } & 146\end{array}$

6.4. Acknowledgements 147

6.5. Experimental Section 147

$\begin{array}{lll}\text { 6.6. } & 150\end{array}$ 


\begin{tabular}{lll}
\hline Chapter 7: & \multicolumn{1}{c}{ Probing Threshold Binding of E. coli to Continuous } \\
& Mannose Gradients under Flow & 159 \\
\hline 7.1. & Introduction & 160 \\
7.2. & Results and Discussions & 162 \\
& 7.2.1. QCM-D Characterization & 162 \\
& 7.2.2. Bacterial adhesion to mannose gradients & 164 \\
7.3. & Conclusions & 169 \\
7.4. & Experimental Section & 169 \\
7.5. & References & 173 \\
7.6. & Supporting Information & 175 \\
\hline
\end{tabular}

Chapter 8: Incorporating bacteria as a living component in 179 supramolecular self-assembled monolayers through dynamic nanoscale interactions

8.1. Introduction 180

8.2. Results and Discussions 183

8.2.1. Detection of the display proteins by SDS-PAGE 183

8.2.2. Testing the display system 184

8.2.3. $\quad \mathrm{CB}[8]$-mediated bacterial aggregation 185

8.2.4. Aggregation kinetics 187

8.2.5. Supramolecular surface adhesion 191

8.3. Conclusions 193

8.4. Acknowledgements 193

8.5. Experimental Section 193

8.6. References 196

$\begin{array}{lll}\text { 8.7. Videos } & 199\end{array}$ 


\begin{tabular}{lll}
\hline Chapter 9: & Epilogue & 201 \\
\hline 9.1. & Introduction & 202 \\
9.2. & $\begin{array}{l}\text { Cell-adhesion force spectroscopy on supramolecular } \\
\text { surfaces }\end{array}$ & 202 \\
9.3. & Supramolecular viral protein cages & 206 \\
9.4. & Bioactive aggregation-induced emission systems & 210 \\
9.5. & Acknowledgements & 213 \\
9.6. & References & 213 \\
\hline
\end{tabular}

Summary 217

Samenvatting 219

Acknowledgements 221

About the Author 224

List of publications 225 


\section{Chapter 1}

\section{The supramolecular bacterial systems toolbox}

Supramolecular chemistry, dealing with systems made up of components that assemble through non-covalent interactions, is currently forming a powerful bridge between chemistry and biology. Though its concepts have been largely derived from natural biological systems, supramolecular chemistry was mostly comprised of complex, dynamic and responsive architectures made up of synthetic components. Eventually biological entities such as peptides, proteins and oligonucleotides were incorporated into such architectures for biomedical applications. Synthetic materials mimicking biological systems were also developed for better integration with cells and tissues. Currently, supramolecular systems comprise of a mélange of synthetic and natural components and are being used to address and study various biological issues. The application of supramolecular chemistry with bacterial systems has so far been sparsely explored. Several powerful possibilities exist in combining these two aspects since bacteria can be addressed either as a research tool or as pathogens. Through genetic engineering, protein engineering and recombinant DNA technologies, they can be used as factories to produce supramolecularly relevant proteinaceous components. On the other hand, they have also evolved as very successful pathogenic agents with intricate molecular mechanisms for invading the human body. Supramolecular chemistry provides powerful methods for developing platforms to study and detect such pathogenic activity. With these in mind, this thesis is an explorative attempt to develop novel strategies and platforms by combining supramolecular chemistry with bacterial systems. This chapter provides a brief introduction to the various components and concepts involved in developing the various systems described throughout this thesis. 


\subsection{Introduction}

Over the past century bacteria have gone from being viewed as purely disease causing pathogens to being used as powerful research, industrial and medical tools. Since the discovery of the first antibiotics in the early twentieth century, vigorous research has been conducted to understand the mechanisms of bacterial pathogenesis and invent strategies to diagnose and cure associated diseases. ${ }^{1}$ During this period several other discoveries like the structure and function of DNA, genes, plasmids, bacterial transformation and restriction endonucleases led to the development of bacteria as a tool for producing recombinant proteins by the 1970 s. $^{2}$ Since then, genetic and protein engineering techniques have rapidly developed and helped to improve our understanding of bacterial pathogenesis at the molecular level. Based on these findings, new approaches to prevent bacterial adhesion, ${ }^{3}$ quorum sensing ${ }^{4}$ and biofilm formation ${ }^{5}$ are being explored as alternatives to antibiotics for curing infections. On the other end of the spectrum, non-pathogenic bacterial strains have been created for the production of proteins with novel properties for research (e.g. fluorescent proteins), ${ }^{6}$ industry (e.g. catalytic enzymes) ${ }^{7}$ and medicine (e.g. insulin). ${ }^{8}$ Synthetic proteins with non-natural properties have also been made by rational design, directed evolution and genetically stitching together different proteins. Furthermore bacterial strains with interesting properties are currently being developed for use as bioactive implant coatings, ${ }^{9}$ biomotors,${ }^{10}$ biosensors, ${ }^{11}$ cell factories ${ }^{12}$ etc. These examples highlight the versatile and important roles played by bacteria in science and society today.

Interestingly, advances in supramolecular chemistry also follow a similar pattern in history. The late nineteenth and early twentieth centuries witnessed the discovery of fundamental supramolecular concepts like non-covalent interactions (van der Waals forces, hydrogen bonds, coordinate bonds, metallic bonds) and molecular recognition (enzymatic lock and key interactions). ${ }^{13}$ These concepts were widely used in chemistry but also played a big role in explaining most biological phenomena involving DNA, RNA, proteins, lipids, carbohydrates etc. During the 1960 s and 1970 s the first macrocyclic host molecules (crown ethers, cavitands, cryptands etc.) were discovered and shown to be able to specifically trap certain types of guest molecules. Since then several other host-guest complexes were developed and in 1987, the term "supramolecular chemistry" was coined when Donald J. Cram, Jean-Marie Lehn and Charles J. Pedersen were awarded the Nobel Prize for it..$^{13}$ From that point onwards, supramolecular architectures became increasingly intricate, resulting in numerous dynamic materials like self-assembled polymers, monolayers, nanostructures and molecular machines. ${ }^{14}$ 
By careful selection of individual components, novel properties like chemical, electrochemical, photochemical, temperature and $\mathrm{pH}$ responsiveness were endowed upon such materials. ${ }^{15}$ This enabled the possibility of using external stimuli to change certain properties of these materials like stiffness, stability, molecular adhesion, size etc. As the molecular interactions in supramolecular chemistry are similar to those found in biological systems, these architectures were soon applied to address and incorporate peptides, proteins, lipids, carbohydrates, living cells etc. ${ }^{16}$ Now, vigorous attempts are being made to use supramolecular chemistry to address and manipulate biological entities at the molecular level for both research and medicine. ${ }^{17,18}$ Biosensors, microarrays, implant coatings, tissue engineering scaffolds and drug delivery vehicles are a few examples of biomedical applications for which supramolecular architectures are currently being developed.

Accordingly, the work in this dissertation represents a collection of studies exploring the possibilities of developing novel systems by combining supramolecular constructs and concepts with bacterial cells and recombinant proteins derived from them.

\subsection{Bacteria}

In this dissertation, we have used Escherichia coli (E. coli), one of the most widely utilized laboratory bacterial strains for studying bacterial adhesion to surfaces, producing recombinant proteins and creating a supramolecularly addressable strain.

\subsubsection{Bacterial adhesion}

Escherichia coli (E. coli), discovered by Theodor Escherich in 1885 , is a gram-negative bacteria commonly found in the intestines of human beings. The cells are rod-shaped with dimensions of $\sim 3 \mu \mathrm{m}$ in length and $\sim 1 \mu \mathrm{m}$ in diameter. ${ }^{19}$ Most strains are highly motile, using uniformly distributed thread-like appendages called flagellae (Figure 1.1) to move at speeds of $20-40 \mu \mathrm{m} / \mathrm{s}^{20}$ Apart from these, they also have smaller hair-like appendages called fimbriae or pili (Figure 1.1), which usually aid in their adhesion to surfaces. ${ }^{21}$ Harmless strains are usually part of the normal gut flora and are symbiotic, providing vitamin $\mathrm{K} 2$ and preventing colonization of invading microbes. There also exist several pathogenic strains that cause problems like gastroenteritis, neonatal meningitis, food poisoning, urinary tract infections etc. In this dissertation we have focused on uropathogenic E. coli (UPEC) and developed supramolecular platforms to study and detect their surface adhesion properties. 
Pathogenic strains of $E$. coli cause infections by forming colonies within internal organs of the body. Such colonization usually begins with the adhesion of bacterial cells to particular tissues in the host organism. In many cases, adhesion occurs through hair-like fimbrial adhesins located on the bacterial surface. ${ }^{21}$ Type 1 fimbrial FimH receptor is one of the most comprehensively studied adhesin in E. coli (Figure 1.1). It aids in the adhesion of UPEC to the walls of the urinary tract, which subsequently forms colonies resulting in urinary tract infections (UTI). FimH binds specifically to mannose sugar molecules found in glycoproteins and glycolipids on the surface of target cells. ${ }^{21}$ This adhesin has been extensively studied since it enables $E$. coli to adhere to surfaces even in the presence of relatively large shear stresses regularly experienced in the urinary tract $\left(>100\right.$ dynes $\left./ \mathrm{cm}^{2}\right) \cdot{ }^{22,23}$ Such robust adhesion seems to be possible due to two important parameters -1 ) the presence of several (200-500) type 1 fimbriae on the bacterial surface allowing for multivalent binding and 2) catch-bonds that bind stronger in the presence of higher shear stresses. ${ }^{24-26}$ Due to these factors, the binding strength of UPEC to cell surfaces depends upon the mannose density and fluid shear stress within the urinary tract. Gaining insights into these parameters would help improve our understanding of the initiation of this bacterial infection and enable the development of appropriate medications to prevent the adhesion.

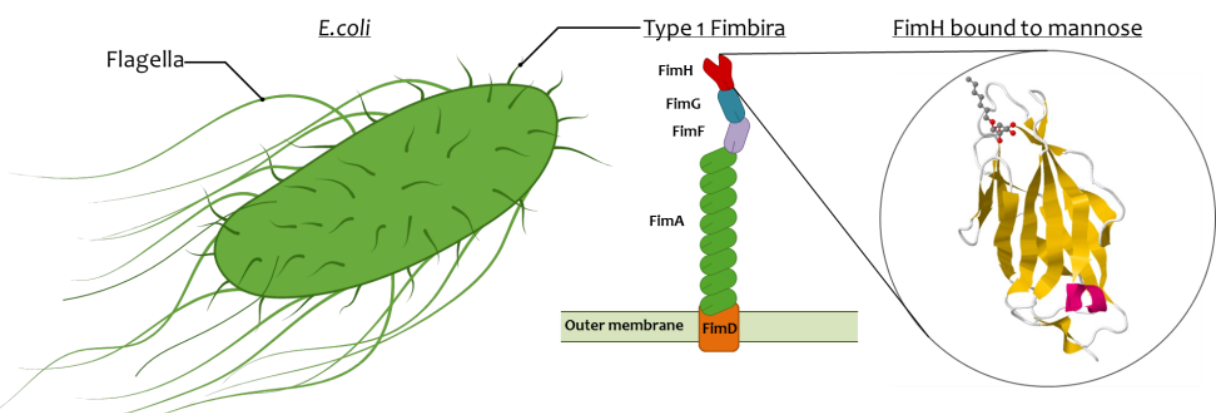

Figure 1.1. Schematic representation of flagellae and type 1 fimbriae on the surface of $E$. coli. The type 1 fimbria is comprised of several fimbrial proteins with the FimH adhesin at the tip capable of binding mannose ligands. The 3D structure of the FimH receptor bound to heptyl $\alpha-D$ mannopyrannoside $e^{27}$ has been reproduced with permission from the RCSB Protein Data Bank with a PDB ID: 4BUQ using Jmol: an open-source Java viewer for chemical structures in 3D, http://www.jmol.org/.

Apart from their natural properties, E. coli also plays a major role in genetic engineering and recombinant DNA technologies. Whole genome sequences of several strains have 
been unraveled, helping us improve our understanding of various genes and their functions. Harmless laboratory strains are used to produce heterologous, fused and even synthetic proteins. Furthermore, genetic modification has led to the creation of strains with new characteristics for research, medical and industrial purposes. We have used this powerful tool to explore the possibility of generating recombinant proteins and even a bacterial strain with multivalent supramolecular functionalities.

\subsubsection{Recombinant DNA technology}

Recombinant DNA is created using laboratory methods to combine genetic material from different sources resulting in sequences that would otherwise not be found in biological organisms. This is possible since all organisms follow the central dogma of molecular biology by which DNA sequences are converted to RNA in a process called 'transcription' and then subsequently into proteins in a process called 'translation'. ${ }^{28}$ There are a total of four different nucleotides that make up any DNA sequence and 20 different amino acids that make up protein sequences. The sequence of nucleotides in a gene eventually gets translated into the amino acid sequence of a protein. This genetic encoding occurs through codons, a sequence of three DNA or RNA nucleotides that corresponds with a specific amino acid or stop signal during protein synthesis. ${ }^{29}$ Since there are 64 possible nucleotide combinations in a codon, most amino acids are encoded by more than one codon sequence. Nevertheless, the codon sequences that correspond to particular amino acids are the same across all organisms. So, if the amino acid sequence of a desired protein is known, it can be heterologously produced in another organism by incorporating the corresponding DNA sequence into its genetic material.

Bacteria are major players in recombinant DNA technology for two main reasons. Firstly, their genetic makeup is relatively simple. In most higher organisms, the genetic sequence for a protein is split up into smaller parts called introns that are separated from one another by exons (non-coding DNA sequences) within the genome. These introns get stitched together when the DNA is converted to mRNA, which is then translated into a protein. ${ }^{30}$ However in bacteria, such introns and exons do not exist and a protein is encoded by a single continuous sequence of nucleotides. Secondly, bacteria have two types of genetic material - the chromosome and plasmids. ${ }^{31}$ The chromosome is the permanent genetic material that encodes for all the vital characteristics of a bacterial cell. Plasmids are relatively temporary smaller circular DNA sequences that usually contain genes required for survival under stressful conditions like abnormal heat, osmotic pressure, $\mathrm{pH}$ and antibiotics. Plasmids are one of the most 
powerful tools in bacterial genetic engineering since they can be modified to carry desired genes into the cell. The act of transporting plasmids into bacterial cells is called 'transformation'. By doing so, bacterial strains that produce desired proteins can be created. ${ }^{31}$

Genes encoding for desired proteins can either be obtained directly from an organism or chemically synthesized. In higher organisms, since genes are usually present in the form of introns and exons within the genome, they are usually obtained by reverse transcription of their corresponding mRNA sequences. In prokaryotes, genes can be directly obtained from the genome. Usually very low quantities of desired genes are obtained either from organisms or by chemical synthesis and they need to be replicated over several orders of magnitude, a process called 'amplification', to obtain a sufficient amount for downstream processes. This amplification is usually done by a technique called Polymerase Chain Reaction (PCR). ${ }^{32}$ The technique uses naturally occurring enzymes called DNA polymerases that "read" template DNA strands, starting from the 5' end and create complementary strands. However, these polymerases cannot create a complementary strand from scratch directly from a template strand, since they can only extend existing DNA strands. So, short DNA strands that correspond to the initial part of a desired sequence, called 'primers', are required to initiate the polymerization. Primers also allow us to selectively amplify genes from a genome, extend strands with functional DNA sequences, introduce mutations and even stitch together different strands.

Once a sufficient quantity of a desired gene is obtained, it needs to be inserted into a plasmid. This is usually done in a "cut and paste" manner using restriction endonucleases and ligases. ${ }^{31}$ Restriction endonucleases are enzymes that can specifically cleave double stranded DNA at or near certain recognition sequences, called restriction sites. This process is called restriction digestion. This cleavage can result in either blunt or sticky ends. Sticky ends arise when the enzyme cuts one of the two DNA strands more than the other. This results in an overhang of one of the strands, allowing it to be available to "stick" to its complementary DNA single strand. So, when the same restriction endonucleases are used to cleave both a gene and a plasmid, they get the same sticky ends, allowing the gene to be "pasted" with the plasmid. This "pasting" is complete only when the DNA backbones are covalently linked, a process called 'ligation', carried out by enzymes called ligases. These enzymes are also used to fuse two or more genes together, eventually resulting in the production of fusion proteins. 
In this dissertation, recombinant proteins are usually produced by first obtaining the desired genes and appropriate plasmids, performing restriction digestion and ligation to insert the genes into the plasmids, transforming the plasmids into a suitable $E$. coli strain and finally inducing the bacteria to express the proteins. Following this, the bacterial cells are made to rupture and the released proteins are purified with purification tags and size exclusion columns. A simplified schematic of the entire process has been depicted in Figure 1.2.

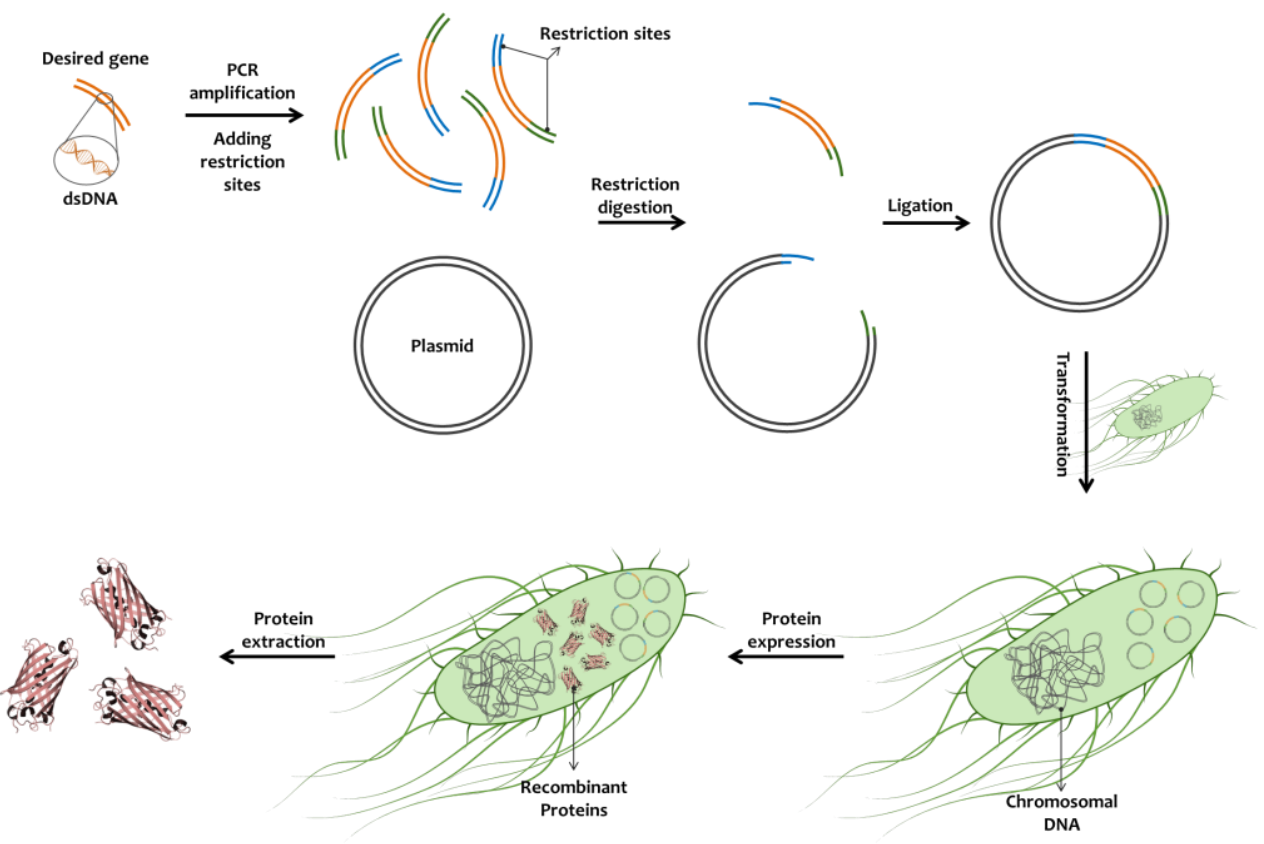

Figure 1.2. Recombinant DNA technologies used to produce a recombinant protein within E. coli.

\subsubsection{Bacteria in nanotechnology and biomedical engineering}

Due to properties like small size, defined shape, rapid growth, motility, robustness, selective adhesiveness and ability to be genetically modified, several studies have explored the utilization of bacterial cells for applications in nanotechnology.

Bacterial motility has inspired their usage as bio-motors for the conversion of chemical energy to mechanical. Strategies to immobilize bacterial cells on substrates in an oriented manner while retaining their motility have been explored for the actuation of micromechanical devices. ${ }^{33}$ Uyeda and coworkers were able to use Mycoplasma mobile 
(M.mobile) to drive a microrotary motor, fueled by glucose (Figure 1.3a). ${ }^{34}$ Fabrizio and coworkers used nanofabrication techniques to construct a microrotor that would be propelled in one direction when dipped in a culture of E. coli. ${ }^{10}$ Recently Sitti and coworkers devised a $\mathrm{pH}$ gradient based system to control multi-bacteria propelled microrobots. ${ }^{35}$ Further advances in improving oriented bacterial adhesion, sustained motility and renewability of the surface could result in effective means of powering microscopic devices using bacterial cells.

Genetic engineering and rapid growth has enabled the development of bacterial strains that can express combinations of heterologous metabolic enzymes as cellular factories capable of synthesizing chemicals for fuels, materials and drugs in an eco-friendly and sustainable manner. ${ }^{36}$ Studies have shown that production yields remain low if the enzymes are freely floating in the cytosol since intermediate products do not get effectively transported from one enzyme to the next. ${ }^{12}$ Furthermore some intermediates can be toxic to the cell or can leak out from the cell. Simultaneous production of protein scaffolds that can assemble the enzymes within close proximity of each other has shown to be extremely effective in solving these issues and improving yields. Currently, research is being conducted to develop robust and multifunctional protein scaffolds for various metabolic systems (Figure 1.3b). ${ }^{12,37}$

Genetic engineering and surface adhesive properties have resulted in the exploration of using bacterial cells as a living interface between synthetic materials and mammalian cells. Salmeron-Sanchez and coworkers have genetically modified a non-pathogenic Lactococcus lactis (L. lactis) to display a fibronectin fragment for the adhesion and differentiation of mammalian cells (Figure 1.3C). ${ }^{9}$ These bacteria were able to colonize material surfaces and form stable biofilms. Such bacterial biointerfaces can be programmed to express desired biochemical signals on demand, establishing a new dimension in biomaterial surface functionalization for biomedical applications.

Bacterial display is another interesting application born out of the ability to genetically modify bacterial strains. Peptides and relatively small proteins of interest can be displayed on the surface of a bacterial cell by genetically fusing them with transmembrane, flagellar and fimbrial proteins. ${ }^{38}$ This system is usually used in applications such as the creation of novel vaccines, the identification of enzyme substrates and finding high-affinity ligands for target proteins through randomized peptide libraries. Daugherty and coworkers have made pioneering progress in this field, identifying and modifying transmembrane proteins for displaying peptides and miniproteins and also optimizing the methodology of the system. ${ }^{39}$ They were able to 8 
identify high-affinity binding peptide sequences for target proteins such as thrombin ${ }^{40}$ and nueropilin, ${ }^{41}$ develop a quantitative kinase $\mathrm{kit}^{42}$ and establish a technique for assessing antibody-epitope specificity. ${ }^{43}$ Apart from displaying peptides and proteins for such research purposes, bacterial display has also been used for eco-friendly utilization of toxic carbon monoxide to make bioplastics. Han and coworkers developed a novel system in which a carbon monoxide-binding protein and carbon monoxide-dehydrogenase were anchored on the cell surface of Ralstonia eutropha ( $R$. eutropha). ${ }^{44}$ The proteins anchored to the cell surface were able to capture and convert carbon monoxide to carbon dioxide. $R$. eutropha was then able to utilize this carbon dioxide in its metabolic pathways to produce poly[(R)-3-hydroxybutyrate] (PHB), a biodegradable and biocompatible, thermoplastic that can be made into films, fibers, and sheets and could potentially be molded into bags and bottles. These examples highlight the versatility of bacterial display systems for developing novel solutions to various problems.

The small size and defined shape of bacteria has enabled their incorporation in biophotonic waveguides. Li and coworkers were able to trap living $E$. coli cells in an oriented manner within biophotonic waveguides up to several tens of micrometers (Figure 1.3d). ${ }^{45}$ They also observed good light propagation through these waveguides and were able to record the propagating signal in real-time. This strategy could enable the generation of a new class of biocompatible microenvironments, merging the optical and biological worlds for real-time detection of signals in biomedical applications.

The few examples described in this section highlight the broad expanse of applications being developed in nanotechnology and biomedical engineering using bacterial systems. 

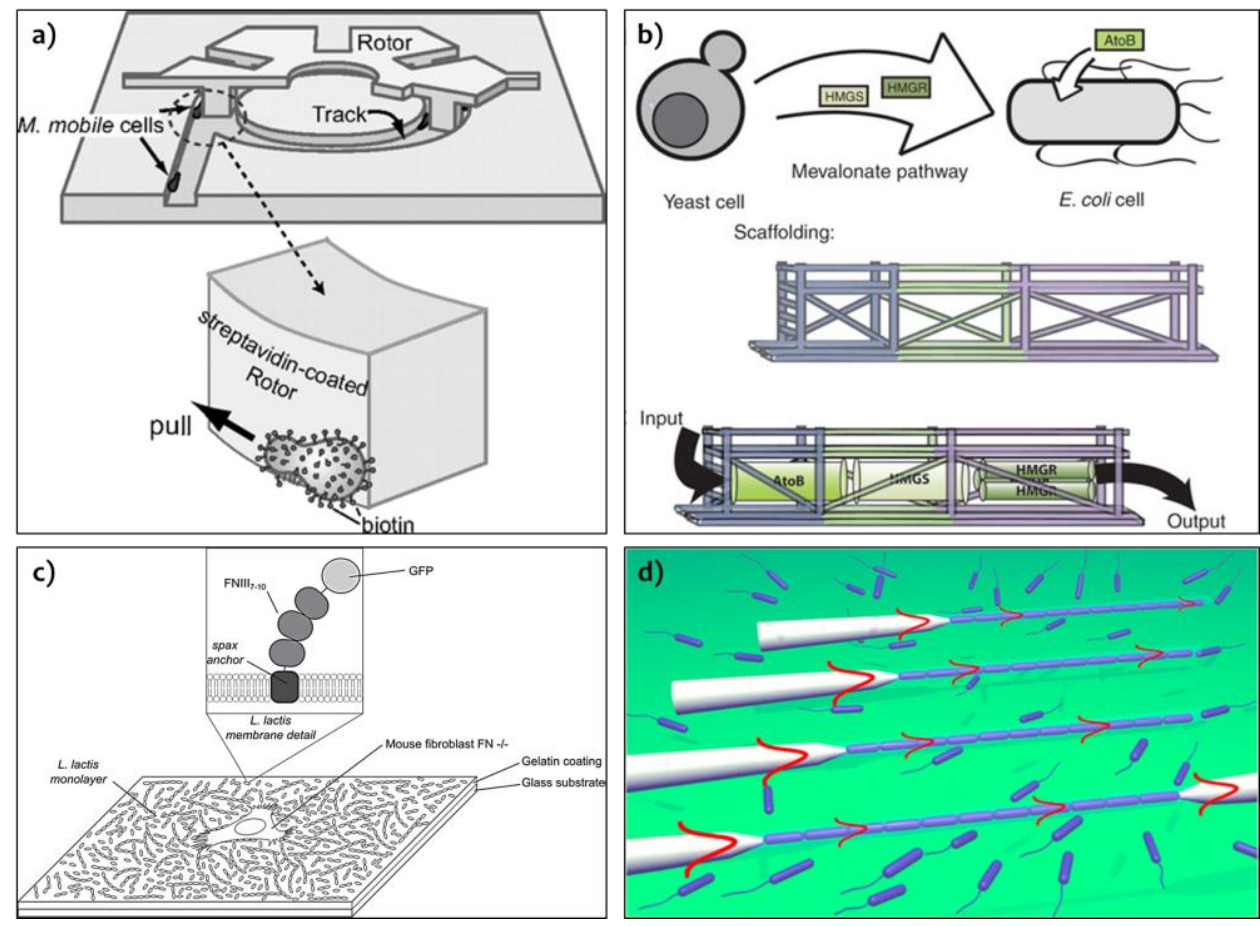

Figure 1.3. Examples of bacteria used in nanotechnology. a) Motility of M. mobile bacterial strain used to propel a microrotor. Reprinted with permission. ${ }^{34}$ Copyright 2006, National Academy of Sciences. b) E. coli used as cellular factories for in vivo production of mevalonate by making them express heterologous metabolic enzymes and a protein scaffold to assemble the enzymes in sequence and improve production efficiency. Reprinted with permission. ${ }^{12}$ Copyright 2009, Nature Publishing Group. c) L. lactis biofilms used as a living interface between synthetic materials and mammalian cells. Reprinted with permission. ${ }^{9}$ Copyright 2013, John Wiley \& Sons. d) Biophotonic waveguides of $E$. coli used to create living microenvironments where signals can be transmitted and recorded in real-time. Reprinted with permission. ${ }^{45}$ Copyright 2013, American Chemical Society.

\subsection{Proteins}

In this dissertation, two major classes of proteins were utilized - fluorescent proteins and cystine-stabilized miniproteins.

\subsubsection{Fluorescent proteins}

The green fluorescent protein (GFP), discovered in the early 1960 s in a jellyfish, is composed of 238 amino acid residues (26.9 kDa) and exhibits bright green fluorescence 
when exposed to light in the blue to ultraviolet range. ${ }^{46}$ The amino acid residues forming the fluorophore are shielded inside a robust $\beta$-barrel resulting in a very high fluorescence quantum yield (up to $80 \%$ ) (Figure $1.4 a$ ). ${ }^{47}$ This tight protein structure also confers resistance to fluorescence variations due to fluctuations in $\mathrm{pH}$, temperature, and denaturants such as urea.

The potential applications of GFP were only realized in the late 1990 s a few years after it was first cloned in bacteria in $1992 .{ }^{48}$ Initially it was used in bacteria and nematodes to follow gene expression but eventually it was fused to antibodies and other proteins that could target particular cellular antigens. Genetic mutation of the fluorophore resulted in modification of its spectral properties, giving rise to differently coloured fluorescent proteins. Several other similar fluorescent proteins were later identified in other species and cloned into bacteria, expanding the existing colour palette. Currently, a broad range of fluorescent proteins exist that feature fluorescence emission spectral profiles spanning almost the entire visible light spectrum (Figure 1.4b). ${ }^{49}$ Today, apart from being used to visualize components in a cell, ${ }^{50}$ these fluorescent proteins are also used to gain insights into protein-protein, protein-ligand and supramolecular host-guest interactions. Using techniques like Fröster resonance energy transfer (FRET), ${ }^{51}$ fluorescence anisotropy, ${ }^{52}$ flow cytometry ${ }^{53}$ and microscale thermophoresis (MST), ${ }^{54}$ detailed information about interaction strengths and orientations can be obtained. 

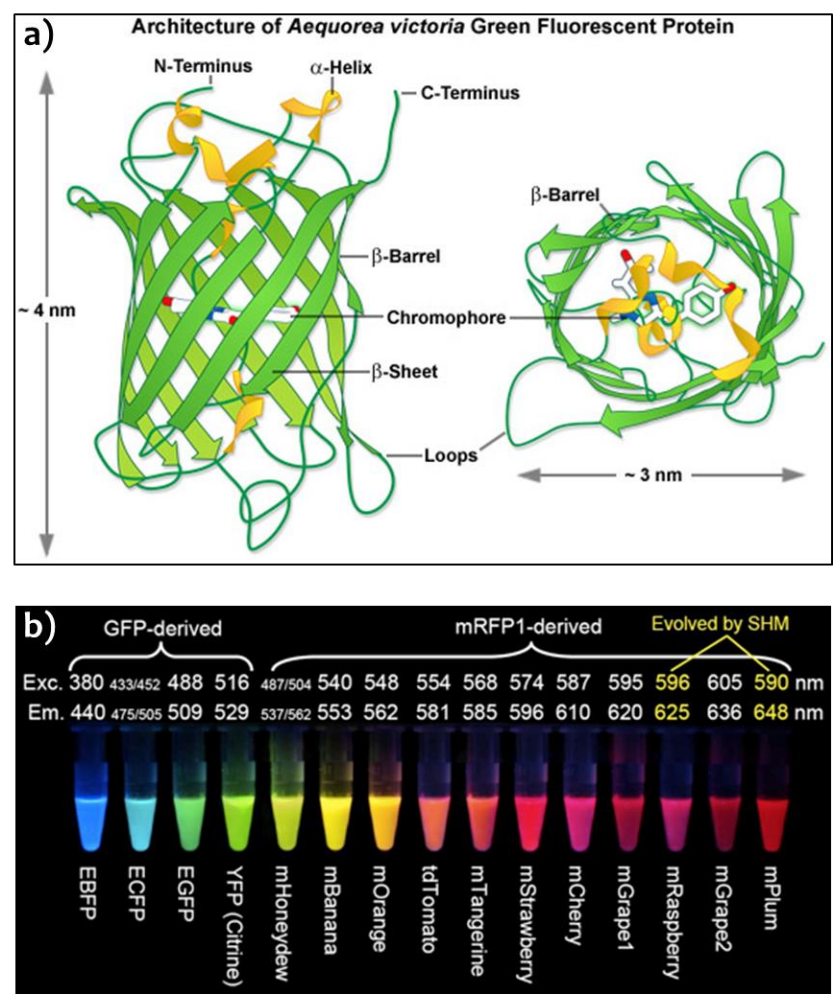

Figure 1.4. a) Structure of GFP (Reproduced with permission from Zeiss Microscopy Online Campus) and $b$ ) different fluorescent proteins currently available with excitation and emission wavelengths spread over the whole visible spectrum. Reprinted with permission. ${ }^{49}$ Copyright 2010, Royal Society of Chemistry. Some are derived from GFP, some from monomeric red fluorescent protein (mRFP) and some were evolved through somatic hypermutation (SHM).

\subsubsection{Cystine-stabilized miniproteins}

Miniproteins like affibodies, kunitz domains, ankyrin repeats, PDZ domains, nanobodies and knottins are currently under intense investigation as alternative non-immunoglobin scaffolds for the generation of novel binding agents. ${ }^{55}$ Amongst these, knottins are one of the smallest naturally-occurring miniproteins (typically 25-35 amino acids) that bind a range of target proteins. ${ }^{56}$ Their three-dimensional structure is essentially defined by a peculiar arrangement of three disulfide bonds. It is made up of a small triple-stranded antiparallel $\beta$-sheet, which is stabilized by the disulfide bond framework. ${ }^{57}$ Due to this structure, knottins are extremely robust with high melting temperatures $\left(T_{m}>100^{\circ} \mathrm{C}\right)$ and even resistant against proteolytic enzymes. Naturally occurring knottins have little 
sequence homology except the set of Cys residues, which gives rise to the conserved pattern of disulfide bridges. ${ }^{57}$ The interspersed peptide loops are highly variable both in length and sequence so that the core of the knottin structure could be a suitable scaffold in order to create interfaces for novel binding activities. ${ }^{57,58}$

The serine protease inhibitor EETI-II, a squash-type inhibitor isolated from jumping cucumber seeds, was the first model of the 'knottin' family. It consists of 28 amino acids and three disulfide bridges, which are essential for the bioactive conformation of this microprotein (Figure 1.5a). ${ }^{58}$ Remarkably, this biomolecule possesses the unfailing ability to refold and correctly form its three disulfide bonds. ${ }^{58}$ Christmann and coworkers established the bacterial production of EETI-II and investigated to what extent this folding motif tolerates modifications to its loop structures..$^{59}$ Using gene synthesis two derivatives were constructed where the six-residue protease-inhibiting loop was either replaced by a 13-residue epitope of Sendai virus L-protein or by a 17residue epitope from the human bone Gla-protein (also called E-tag). Both wild-type EETI-II and its variants were produced in a correctly folded state - despite being contaminated with disulfide-crosslinked oligomers - at high yields (10-20 mg/L culture after IMAC purification) via secretion into the periplasm of E. coli as fusions with the maltose-binding protein (MalE). The cysteine residues were shown to be fully oxidized and the engineered knottins were binding to monoclonal antibodies directed against the epitopes. A large number of cystine-stabilized miniproteins have also been identified to possess analgesic, anthelmintic, antimalarial, antimicrobial, antitumor, insecticide, protease inhibitory and toxic properties. An open-access online knottin database (http://knottin.cbs.cnrs.fr) has been created by Gracy and coworkers providing standardized information on these small disulfide-rich knotted proteins. ${ }^{60}$ So far, 2193 sequences from 450 organisms with 167 experimentally obtained 3D structures have been listed. Binding affinities of these inhibitor cystine knots have been determined to be in the low $\mathrm{nM}$ ranges. Cochran and coworkers, through rational design strategies, developed knottins containing RGD with flanking residues that were able to bind different integrins with different levels of affinity. ${ }^{61}$ Daugherty and coworkers used bacterial display systems with loop-modified knottins to identify high affinity inhibitors of thrombin ${ }^{40}$ and neuropilin. ${ }^{41}$ Chiche and coworkers developed a truncated 23 residue form of the knottin EETI-II, called Min-23, with only two sets of stabilizing disulfide bridges (Figure 1.5b). ${ }^{62}$ Similar to its parent knottin, it is comprised of a cystine-stabilized $\beta$-sheet (CSB) motif that forms an autonomous folding unit with three $\beta$-strands and a short $\alpha$-helix. Even though Min-23 miniproteins are stabilized by only two disulfide bridges they still retain high melting temperatures $\left(T_{m} \sim 100^{\circ} \mathrm{C}\right)$ and 
proteolytic resistance. The peptide sequence of the second $\beta$-turn is admissible to extensive modifications, up to 10 amino acids, for which high affinity binders have been identified for various target proteins like VEGF, HIV-Nef, mitochrondiral membrane protein Tom70 etc. ${ }^{63,64}$ Further, to expand upon the functionality of the exposed groups in these cystine-stabilized miniproteins, strategies have been developed to chemically conjugate various synthetic molecules and non-natural amino acids. ${ }^{61,65}$ Such constructs have been successfully shown as effective imaging agents using conjugated fluorescent dyes and radiofluorinated compounds (Figure 1.5C)..$^{8}$ These cystinestabilized miniproteins have thus developed into peptide species which are extremely attractive for synthetic biology purposes since they are small, easily accessible to chemical synthesis, stable, high-affinity binders and tolerate extensive sequence modifications.
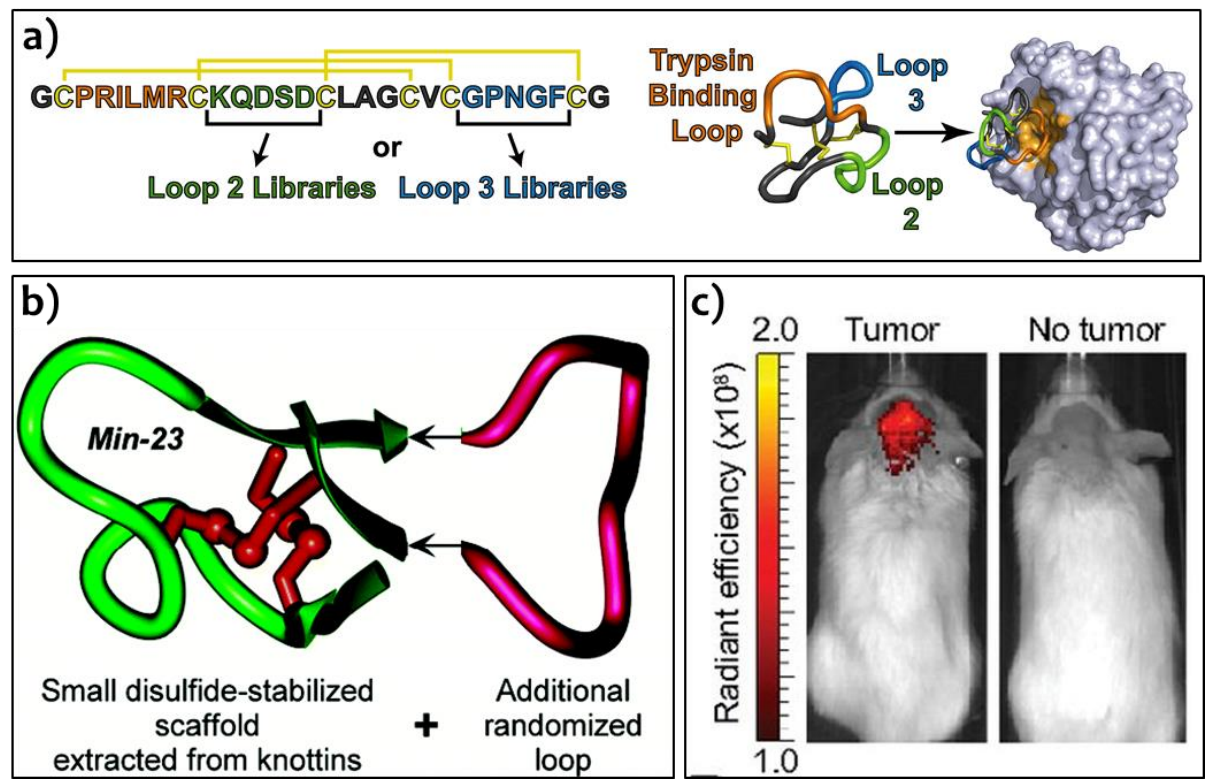

Figure 1.5. a) Sequence, structure and 8-trypsin binding of EETI-II knottin. Reprinted with permission. ${ }^{58}$ Copyright 2009, Lahti et al. The trypsin binding loop (orange), two other modifiable loops (green, blue) and disulfide bridges (yellow) have been shown. b) 3-D structure of Min-23 miniprotein developed by eliminating the trypsin-binding loop from EETI-II knottin. The modifiable loop has been indicated in pink. Reprinted with permission. ${ }^{63}$ Copyright 2005, American Chemical Society. c) AF680-labeled integrin-binding engineered knottin illuminates mouse medulloblastoma in vivo imaged 2 hrs after tail vein injection (mouse with (left) and without (right) tumor). Reprinted with permission. ${ }^{61}$ Copyright 2013, National Academy of Sciences. 


\subsection{Supramolecular host-guest chemistry}

Host-guest chemistry is a branch of supramolecular chemistry that involves binding through molecular recognition caused by non-covalent interactions, such as ion-ion, ion-dipole, dipole-dipole, hydrogen bonding, cation- $\pi$, anion- $\pi, \pi-\pi$, closed shell and van der Waals interactions. In general the host is a bigger molecule that possesses convergent binding sites (containing a pocket) and a guest is a smaller entity that possesses divergent binding sites (able to sit in the pocket). Such systems are analogous to protein-ligand interactions. The host and guest molecules used in this dissertation are described in this section.

\subsubsection{Hosts}

\section{Cyclodextrins}

Cyclodextrins (CDs) are a structurally related family of natural products formed during bacterial digestion of cellulose. ${ }^{66}$ These cyclic oligosaccharides, consisting of $(a-1,4)$ linked $a$-D-glucopyranose units, contain a slightly hydrophobic central cavity and a hydrophilic outer surface. Due to the chair conformation of the glucopyranose units, the cyclodextrins are shaped like a truncated cone rather than perfect cylinders (Figure 1.6a). The hydroxyl groups are on the rims and are oriented to the cone exterior with the primary hydroxyl groups of the sugar residues at the narrow edge of the cone and the secondary hydroxyl groups at the wider edge. The secondary hydroxyl groups are linked through hydrogen bonds, resulting in the stiffening of the truncated cone. The central cavity contains the skeletal carbons and ethereal oxygens of the glucose residues, resulting in its slightly hydrophobic nature. ${ }^{66}$

The natural $\alpha$-, $\beta$ - and $\gamma$-cyclodextrins ( $\alpha-C D, \beta-C D$ and $\gamma-C D$ ) consist of six, seven, and eight glucopyranose units, respectively. Apart from their natural form, there are also several chemically modified CDs, achieved by substitution of the hydroxyl groups. Functionalities that alter the specificity, physical and chemical properties of these CDs have been introduced for various applications. ${ }^{67,68} \mathrm{CDs}$ can bind a wide range of hydrophobic compounds and are used to improve their water solubility and also mask undesirable properties such as odor and taste (Figure 1.6b). ${ }^{69}$ Such CD-based complexes are currently used in pharmaceutical, food and cosmetic industries. CDs are also widely used in research for catalysis, drug delivery, molecular machines, polymers, surface modification, dynamic vesicles, etc. ${ }^{68,69}$ 
a)

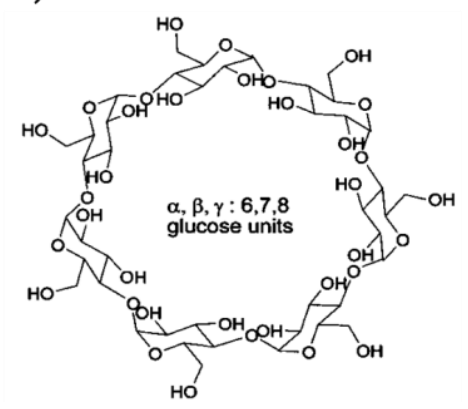

b)

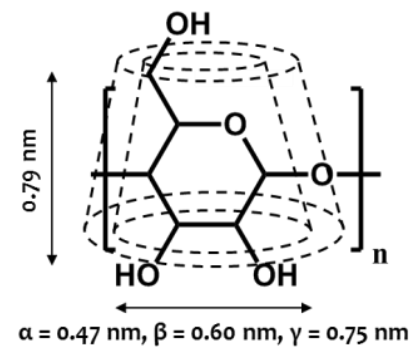

c)

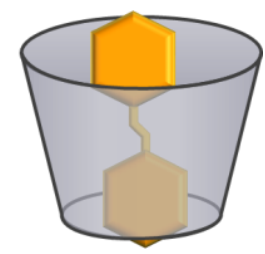

Figure 1.6. a) Chemical structure of 6 -cyclodextrin. Reprinted with permission. ${ }^{70}$ Copyright 2005, Royal Society of Chemistry. b) Schematic representation of the 3D structure with height of the truncated and cavity diameters given. c) Incorporation of a hydrophobic molecule, azobenzene (yellow), within the hydrophobic cavity.

\section{Cucurbit[n]urils}

Cucurbit[n]urils ( $\mathrm{CB}[\mathrm{n}])$ are hollow pumpkin-shaped macrocyclic molecules made of glycouril monomer units. ${ }^{71}$ They have a hydrophobic cavity and polar rims lined with ureido-carbonyl oxygens allowing them to bind a variety of aromatic cations with micromolar affinities. The size of the cavity depends on the number of glycouril units and accordingly, there are $\mathrm{CB}[\mathrm{n}] \mathrm{s}$ of different sizes (Figure 1.7a,b): cucurbit[5]uril $(\mathrm{CB}[5])$, cucurbit $[6]$ uril $(\mathrm{CB}[6])$, cucurbit[7]uril $(\mathrm{CB}[7])$, cucurbit $[8]$ uril $(\mathrm{CB}[8])$ and cucurbit[10]uril $(\mathrm{CB}[10]) .^{72,73}$ Depending on the size, various guest molecules can fit within the cavity with different affinities. Among these, $\mathrm{CB}[8]$ happens to be especially interesting since the cavity is large enough to simultaneously encapsulate two aromatic guest molecules. ${ }^{71,72}$ Homo- and hetero-ternary complexes comprising molecules like methylviologen, azobenzene, aromatic amino acids and naphthol have been shown to form with stabilizing charge-transfer interactions between the two guests in the cavity (Figure 1.7C). ${ }^{74}$ Dynamic and responsive polymers, surfaces, nanostructures and hydrogels have been constructed using $\mathrm{CB}[8]$ and its guests for various applications like tissue engineering scaffolds, ${ }^{75}$ cellular manipulation platforms, ${ }^{76,77}$ biomolecule detection, ${ }^{78,79}$ drug delivery ${ }^{80}$ etc. 

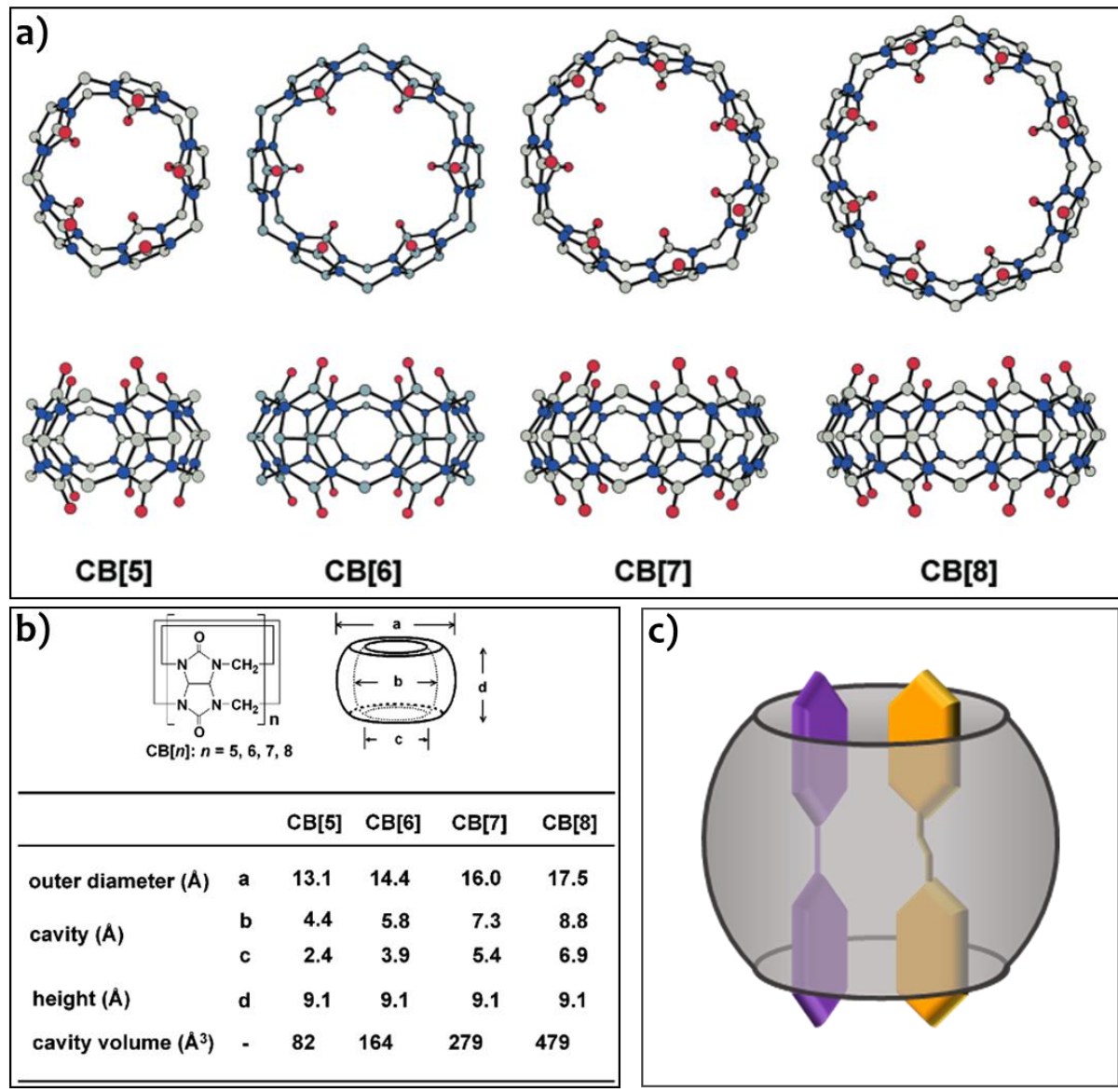

\begin{tabular}{|c|c|c|c|c|c|}
\hline $\mathrm{CB}[n]: n=$ & 5,6, & & $F c-1$ & $\begin{array}{l}\uparrow \\
d \\
\downarrow\end{array}$ & \\
\hline & & $\mathrm{CB}[5]$ & $\mathrm{CB}[6]$ & Св[7] & $\mathrm{CB}[8]$ \\
\hline outer diameter $(\hat{A})$ & a & 13.1 & 14.4 & 16.0 & 17.5 \\
\hline \multirow{2}{*}{ cavity (A) } & b & 4.4 & 5.8 & 7.3 & 8.8 \\
\hline & c & 2.4 & 3.9 & 5.4 & 6.9 \\
\hline height ( $A$ ) & d & 9.1 & 9.1 & 9.1 & 9.1 \\
\hline cavity volume $\left(\AA^{3}\right)$ & - & 82 & 164 & 279 & 479 \\
\hline
\end{tabular}

c)

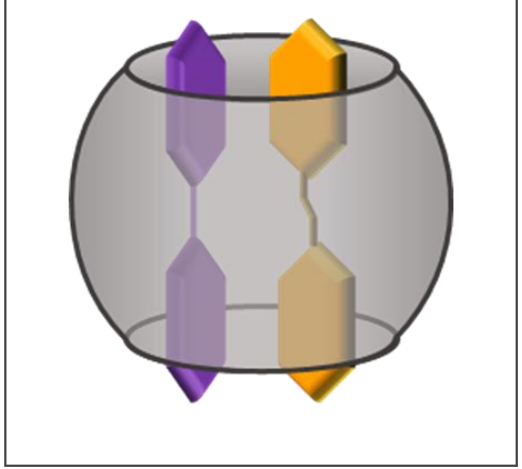

Figure 1.7. a) 3D crystal structures of some cucurbit [n] urils. Reprinted with permission. ${ }^{72}$ Copyright 2003, American Chemical Society. b) Chemical structure and dimensions of cucurbit [n] urils. Reprinted with permission. ${ }^{72}$ Copyright 2003, American Chemical Society. c) Schematic representation of the inclusion of two aromatic guests, methylviologen (purple) and azobenzene (yellow) within the hydrophobic cavity of $\mathrm{CB}[8]$.

\subsubsection{Guests}

\section{Methylviologen}

Methylviologen $\left(\mathrm{MV}^{2+}\right)$, also known as paraquat, is a positively-charged redox-active heterocyclic dipyridium compound. It has been widely used as an electron-acceptor and electron-transfer indicator in the studies of biological, chemical and photochemical redox reactions. ${ }^{81} \mathrm{MV}^{2+}$ can undergo two successive and reversible one-electron 
reductions, first forming a radical cation species $\left(\mathrm{MV}^{+}\right)$and then a neutral quinoid form (MV) (Figure 1.8). ${ }^{82}$ Even though $\mathrm{MV}^{2+}$ does not bind cyclodextrins, the doubly reduced $M V$ form has been shown to weakly interact with $\beta-C D$ with sub-millimolar affinity (Figure 1.8) ${ }^{82}$ However the opposite is true with $\mathrm{CB}[7]$ and $\mathrm{CB}[8]$ where $\mathrm{MV}^{2+}$ has been shown to bind strongly with low micromolar affinities (Figure 1.8). ${ }^{83,84}$ The reduction of $\mathrm{MV}^{2+}$ to $\mathrm{MV}^{+}$and $\mathrm{MV}$ forms results in progressively lower affinities towards $\mathrm{CB}[7] .{ }^{83} \mathrm{In}$ the case of $\mathrm{CB}[8], \mathrm{MV}^{2+}$ binds in a 1:1 ratio and the radical cation $\mathrm{MV}^{+.}$forms a stable dimer within the $\mathrm{CB}[8]$ cavity with a dimerization constant of $2 \times 10^{7} \mathrm{M}^{-1}$ (Figure 1.8). ${ }^{84}$ Acting as an electron acceptor, $\mathrm{MV}^{2+}$ can also form stable charge-transfer complexes with electron donating aromatic guests like azobenzene, naphthol, tryptophan and phenylalanine inside the $\mathrm{CB}[8]$ cavity (Figure 1.8)..$^{71,72,74}$ Reduction of $\mathrm{MV}^{2+}$ to the radical cation form results in destabilization of the charge-transfer complexes, usually causing the second guest to get expelled from the cavity. ${ }^{76,85,86}$ Such supramolecular complexes with $\mathrm{MV}^{2+}$ and $\mathrm{MV}^{+}$have been used to make dynamic and responsive polymers, ${ }^{87}$ surface coatings, ${ }^{76,77,79}$ nanoparticles ${ }^{88}$ and hydrogels. ${ }^{89}$

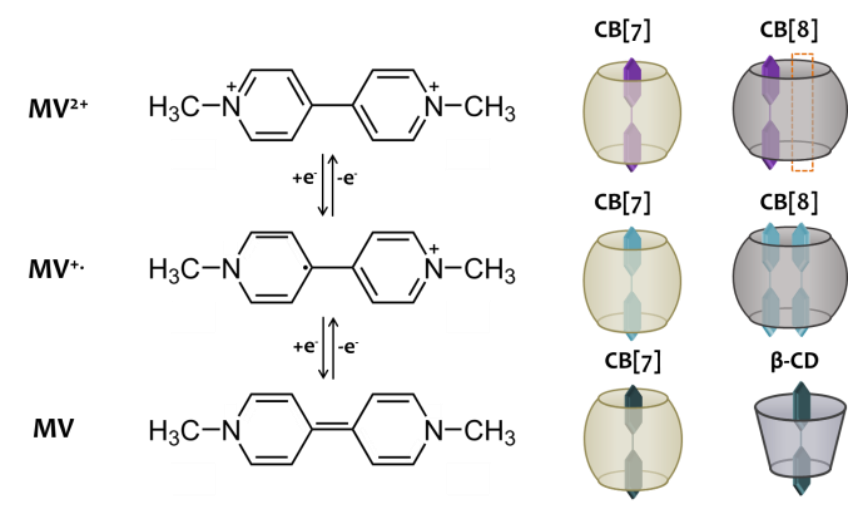

Figure 1.8. a) Different oxidation states of methylviologen along with known supramolecular complexes formed with $\mathrm{CB}[8], \mathrm{CB}[7]$ and $\mathrm{B}-\mathrm{CD}$. In the case of $\mathrm{CB}[8]-\mathrm{MV}^{2+}$, a second different guest molecule can be included within the cavity represented by an orange dashed box.

\section{Azobenzene}

Azobenzene (azo) is composed of two phenyl rings connected by a diazene (- $\mathrm{HN}=\mathrm{NH}-)$ bond. A wide range of molecules have been derived from this core structure by extending the phenyl rings with chemical functionalities..$^{90}$ One of the most interesting properties of azo and its derivatives is their ability to photo-isomerize from the more stable trans to the relatively unstable cis form on irradiation with light in the ultraviolet 
(UV) regime (300 - $400 \mathrm{~nm}$ wavelengths) (Figure 1.9). ${ }^{90}$ Visible light (>400 nm wavelength) can be used to convert the molecule back to the trans form. Alternately, if no irradiation is applied, thermal back-relaxation to the stable trans form will slowly occur. The trans form is known to bind $\alpha-C D, \beta-C D, C B[7]$ and the $C B[8]-M^{2+}$ complex with moderate micromolar affinities (Figure 1.9). ${ }^{85,87,91-93}$ Upon photo-isomerization, the cis form experiences a weaker affinity with these $C D$ cavities due to steric constraints. ${ }^{91}$ With $\mathrm{CB}[7]$, the isomerization to cis-azo is known to destabilize the complex ${ }^{92}$ but certain azo derivatives are known to bind even stronger in their cis form. ${ }^{93}$ It has even been shown that $\mathrm{CB}[7]$ induces trans-cis isomerization of such derivatives under certain conditions. ${ }^{93}$ In the case of photo-isomerization of the trans-azo-CB[8]-MV ${ }^{2+}$ ternary complex, since the cis-azo isomer can solely occupy the entire cavity, either $\mathrm{MV}^{2+}$ or cisazo gets expelled from the cavity depending upon the type of azo derivative used. ${ }^{85,87}$ The cis isomer of unmodified azo does not expel $\mathrm{MV}^{2+}$ from within the cavity ${ }^{85}$ however a pyridium-functionalized azo (Figure 1.9) in its cis form is able to bind $\mathrm{CB}[8]$ with a higher affinity than $\mathrm{MV}^{2+}$, resulting the expulsion of $\mathrm{MV}^{2+}$ from the cavity. ${ }^{87}$ Thus cis isomers of azo derivatives that are able to form complexes with $\mathrm{CB}[7]$ and $\mathrm{CB}[8]$ have functionalizations that form stabilizing interactions with the polar rims of these host molecules (Figure 1.9). These photo-switchable host-guest complexes have been used to construct optically responsive polymers, ${ }^{87}$ surfaces, ${ }^{94}$ hydrogels, ${ }^{91}$ molecular machines ${ }^{95}$ and nanoparticles. ${ }^{88}$ 

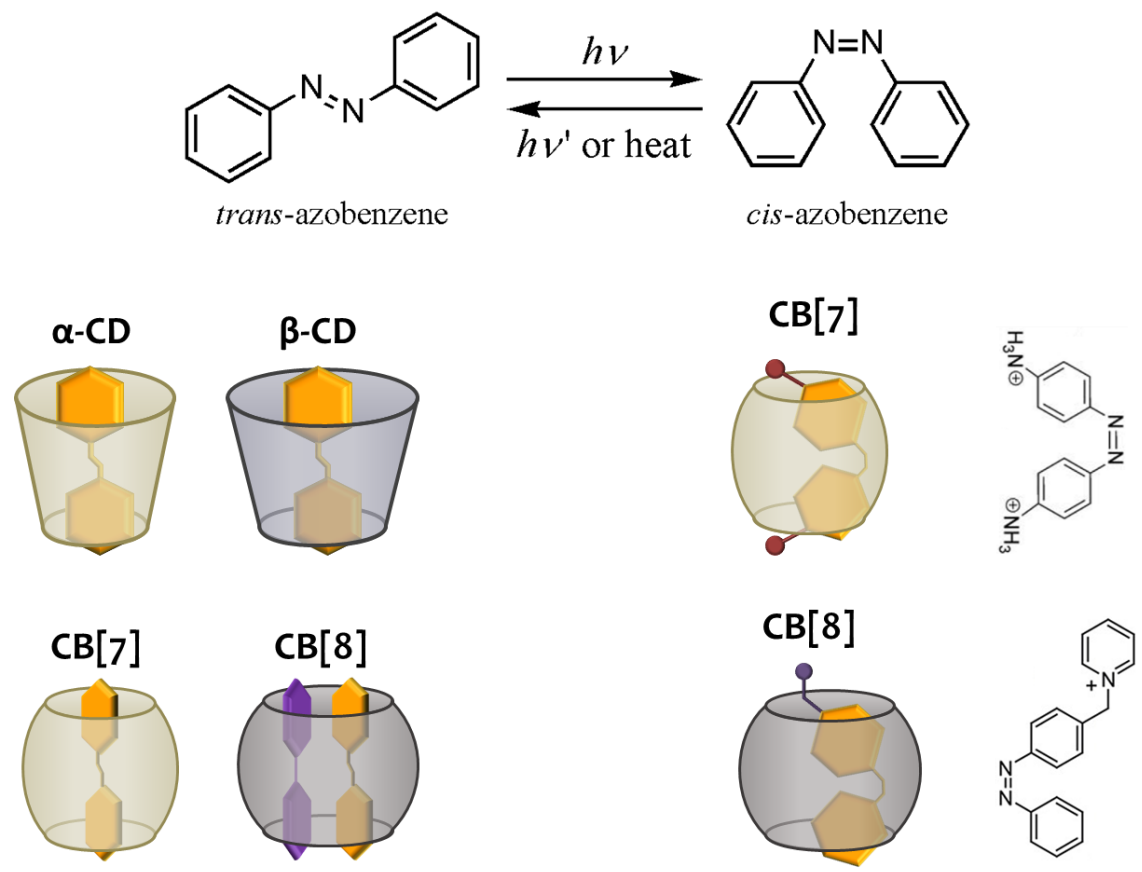

Figure 1.9. Azobenzene isomers along with schematics representing a few known inclusion complexes with $\alpha-C D, b-C D, C B[7], C B[8]-M V$ and $C B[8]$. For the cis isomer, chemical structures of azo derivatives that are known to form stable complexes with $C B$ [7] and $C B$ [8] have been included on the right.

\section{Peptide motifs}

Aromatic amino acids such as tryptophan, tyrosine and phenylalanine have been shown to bind $C D$ and $C B[n]$ s over a wide range of affinities $\left(K_{d}=10^{-2}-10^{-5} \mathrm{M}\right)$. Binding usually occurs with the aromatic side chains entering the hydrophobic cavities and the amino acid backbones sometimes interacting with the rims of the macrocyclic hosts. Weak binding of such aromatic amino acids either in their free form or as part of peptides has been shown to occur with $\alpha$ - and $\beta$-CDs with low to moderate millimolar affinities. ${ }^{96-98}$ Higher affinities have been achieved with linear and branched constructs containing multiple weak-binding aromatic amino acids when allowed to bind in a multivalent manner with $\beta$-CDs arrays on surfaces. ${ }^{99}$ Based on such interactions with aromatic amino acids, CDs have been used in solution to improve the solubility and stability of proteins and drugs that carry these amino acids. ${ }^{66-68}$ On the other hand, $C B[n] s$ bind hydrophobic amino acids with appreciable affinities in the low to mid micromolar 
regime. The relatively smaller hydrophobic amino acids of alanine and valine have been shown to occupy the cavity of $\mathrm{CB}[6] .^{100}$ The aromatic amino acids of phenylalanine (F), tryptophan $(\mathrm{W})$ and tyrosine $(\mathrm{Y})$ have been shown to bind $\mathrm{CB}[7]^{101}$ and the $\mathrm{CB}[8]-\mathrm{MV}^{2+}$ complex ${ }^{102}$ with 1:1 stoichiometry. With $\mathrm{CB}[8]$, these amino acids form homoternary complexes with two aromatic groups simultaneously occupying the cavity and being stabilized by $\pi-\pi$ interactions between them. ${ }^{103}$ Interestingly, in peptides, the $\mathrm{N}$ terminal aromatic amino acids have a significantly higher affinity towards $\mathrm{CB}[7]$ and $\mathrm{CB}[8]$ compared to $\mathrm{C}$-terminal or internal positions. ${ }^{101-103}$ It has been proposed that this is due to the stabilizing interaction of the charged $\mathrm{N}$-terminal amino group with the polar ureido-carbonyl oxygens on the rims of these $\mathrm{CB}[\mathrm{n}] \mathrm{s}$. On the other hand, $\mathrm{C}$ terminal carboxyl groups contribute destabilizing repulsive interactions with the rims. It has also been shown that the binding strengths of peptides containing these amino acids depend upon adjacent amino acids in the sequences. ${ }^{102-104}$ Currently, peptide motifs like FGG, WGG, GGWGG and GGFGG have been used to construct supramolecular polymers, ${ }^{105}$ induce protein dimerization, ${ }^{106,107}$ facilitate adhesion of peptides and proteins to surfaces, ${ }^{76,77,79}$ hydrogels, ${ }^{108}$ etc. The various peptide motifs used as guests in this thesis and their corresponding host molecules have been shown in Figure 1.10.

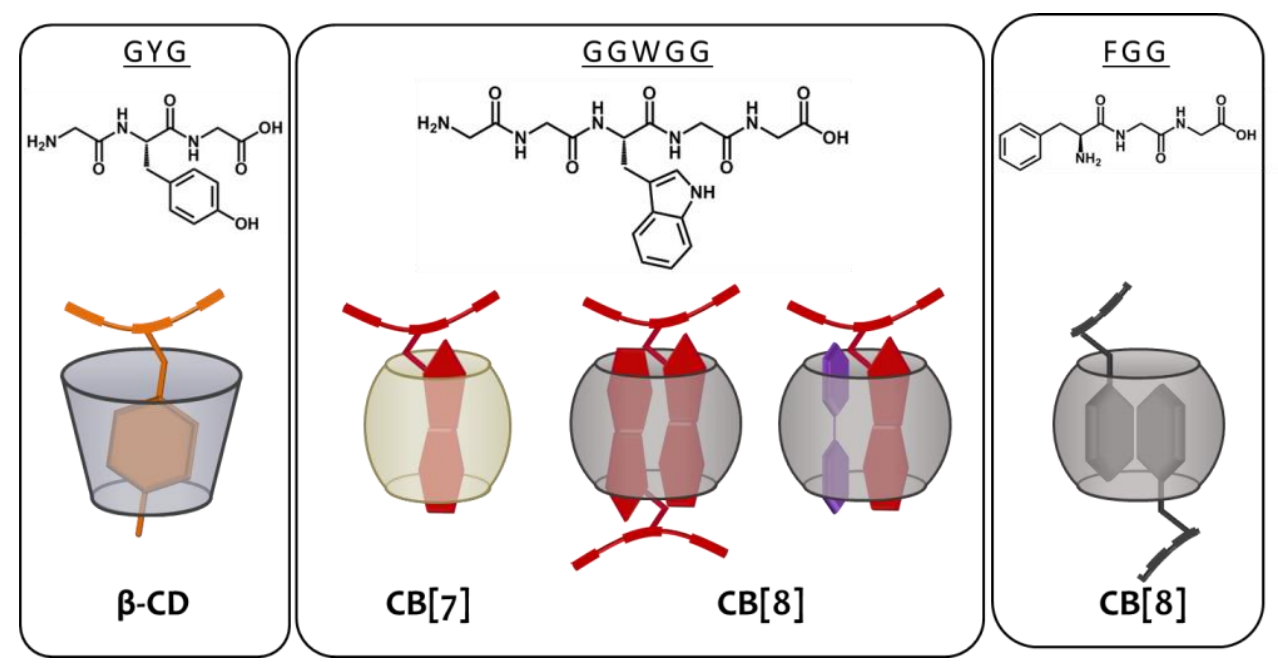

Figure 1.10. Schematic displaying the supramolecular peptide motifs used as guest molecules in this thesis along with their corresponding hosts. Tyrosine (Y), tryptophan (W) and phenylalanine (F) have been used as part of the peptide sequences GYG, GGWGG and FGG respectively. These peptide sequences were also fused with fluorescent proteins and miniproteins. 


\subsubsection{Stimuli Responsiveness}

Since non-covalent interactions exist in a state of equilibrium, environmental factors can influence whether the system exists in a bound or unbound state. For instance, hydrophobic interactions, like the binding of aromatic guests within the cavity of CDs, are usually favoured in aqueous solvents. Charge transfer complexes between $\mathrm{MV}^{2+}$ and another aromatic guest such as naphthol, azobenzene, aromatic amino acids etc. within the cavity of $\mathrm{CB}[8]$ gets destabilized once $\mathrm{MV}^{2+}$ is reduced to $\mathrm{MV}^{+}$. The binding affinities of azobenzenes within the cavities of $C D s$, and $C B[n] s$ can be changed by photoisomerization between their cis and trans forms. A guest bound within the cavity of a host can be replaced by another guest that has a higher affinity towards the host molecule. These examples show that it is possible to change the properties of certain supramolecular systems by changing environmental factors. These factors like light, temperature, $\mathrm{pH}$, electrical potential, etc. that we can control are termed stimuli and the ability of a system to be modified by these stimuli is called stimuli responsiveness (Figure 1.11). This provides us with the possibility of developing systems over which we not only have spatial control by also temporal control.

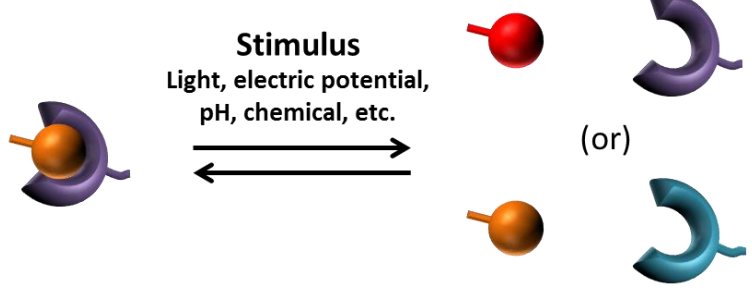

Figure 1.11. The basis of stimuli responsiveness. Upon external stimulus, binding entities either undergo binding or unbinding due to a change in either the ligand or the binding site. By constructing materials with these components, stimuli-reponsiveness can be endowed upon the whole material.

By careful selection and synthesis of molecular components, different types of supramolecular architectures have been developed that respond to various stimuli. Supramolecular polymers have been developed that can switch from liquid to solid state or vice versa by changing the temperature, solvent properties, chemical constituents, applying light of a certain wavelength, electric potentials and even mechanical stress. ${ }^{15,109}$ Such polymers have potential applications in adhesives, cosmetics, printing and material coatings. Soft nanoparticles, held together by monovalent and multivalent supramolecular components have been designed that are size-tuneable based on their chemical composition. ${ }^{110}$ Photo-responsive and redox- 
active components have also been used such that these nanoparticles can be disassembled by applying the appropriate stimulus. ${ }^{111}$ Such nanoparticles have great potential as drug carriers for targeted drug delivery. Supramolecular hydrogels that can swell, contract, become softer or stiffer and undergo sol-gel transitions depending on the external stimulus have been developed for various biomedical applications such as smart medical implants, controlled drug delivery, tissue regeneration scaffolds etc. ${ }^{112,113}$ Stimuli responsive-surface coatings have been widely developed to study biological phenomena that occur due to changes in the environment. Strategies to assemble supramolecular hosts and guests on surfaces allowing the possibility to switch them between being cell adhesive and cell repellent have led to insightful discoveries about biomechanical and chemical changes that occur within cells during adhesion and release from surfaces. ${ }^{76,77,94,114}$ Such surfaces are also being developed to make responsive biomedical implant coatings, reusable biosensors etc. Thus stimuliresponsiveness provides us additional spatial and temporal control over the materials we design, allowing us to develop extremely versatile systems.

\subsection{Outline of the thesis}

This thesis covers an explorative approach in combining the components and concepts described in this chapter. The work described in this thesis has been organized in three sections -1 ) using bacteria to produce recombinant supramolecularly relevant proteins (Chapters 2-4), 2) developing supramolecular platforms to address bacteria as pathogens (Chapters 5-7) and 3) using the bacterial cell as a supramolecular entity (Chapter 8). In Chapter 2, a $\beta$-CD-binding multivalent peptide tag was designed and its binding properties on $\beta-C D$ monolayers were studied. This tag was then genetically fused to a red fluorescent protein and its ability to immobilize the protein on $\beta-C D$ monolayers was studied. In Chapter 3, a strategy was developed to construct functional knottin-displaying surfaces by genetically fusing a $\mathrm{CB}$ [8]-binding peptide motif to its $\mathrm{N}$ - and C-terminals. In Chapter 4, a strategy to develop multivalent and multispecific proteins was tested by genetically engineering chains of miniprotein scaffolds. In Chapter 5, a photoresponsive platform for studying the adhesion of proteins and bacteria was developed using $\beta-C D$ monolayers and azobenzene glycoconjugates. In Chapter 6, a similar, yet improved platform was developed using ternary complexes of $\mathrm{MV}^{2+}, \mathrm{CB}[8]$ and an azobenzene glycoconjugates immobilized on supported lipid bilayers. In Chapter 7, gradients of mannose were made on solid state supported lipid bilayers in microchannels and were used to study the effect of mannose surface density and solution shear stress on the binding capabilities of $E$. coli. In 
Chapter 8 a supramolecularly addressable bacterial strain was developed and incorporated as a living component in supramolecular SAMs. The thesis concludes with an epilogue in which possible future avenues of using these supramolecular components are explored and concepts to develop and analyze systems that are outside the major scope of this thesis are discussed. One avenue explores the possibility of using FluidFM to study cell adhesion forces on supramolecular surfaces, second avenue investigates the possibility of introducing supramolecular functionality to viral protein cages and a final avenue tests the possibility of using an aggregationinduced-emission system for labelling bacteria.

\subsection{References}

(1) Zaffiri, L., Gardner, J., and Toledo-Pereyra, L. H. (2012) History of antibiotics. From salvarsan to cephalosporins. J. Invest. Surg. 25, 67-77.

(2) Wright, S. (1986) Recombinant DNA technology and its social transformation, 1972-1982. Osiris 303-360.

(3) Zhu, X. Y., Holtz, B., Wang, Y., Wang, L.-X., Orndorff, P. E., and Guo, A. (2009) Quantitative glycomics from fluidic glycan microarrays. J. Am. Chem. Soc. 131, 13646-13650.

(4) Hentzer, M., and Givskov, M. (2003) Pharmacological inhibition of quorum sensing for the treatment of chronic bacterial infections. J. Clin. Invest. 112, 1300-1307.

(5) Smith, A. W. (2005) Biofilms and antibiotic therapy: Is there a role for combating bacterial resistance by the use of novel drug delivery systems? Mech. Antimicrob. Resist. Oppor. New Target. Ther. 57, 1539-1550.

(6) Chudakov, D. M., Matz, M. V., Lukyanov, S., and Lukyanov, K. A. (2010) Fluorescent proteins and their applications in imaging living cells and tissues. Physiol. Rev. 90, 1103-1163.

(7) Koeller, K. M., and Wong, C.-H. (2001) Enzymes for chemical synthesis. Nature 409, 232-240.

(8) Johnson, I. (1983) Human insulin from recombinant DNA technology. Science 219, 632-637.

(9) Saadeddin, A., Rodrigo-Navarro, A., Monedero, V., Rico, P., Moratal, D., González-Martín, M. L., Navarro, D., García, A. J., and Salmerón-Sánchez, M. (2013) Functional living biointerphases. Adv. Healthc. Mater. 2, 1213-1218.

(10) Di Leonardo, R., Angelani, L., Dell'Arciprete, D., Ruocco, G., lebba, V., Schippa, S., Conte, M. P., Mecarini, F., De Angelis, F., and Di Fabrizio, E. (2010) Bacterial ratchet motors. Proc. Natl. Acad. Sci. 107, 9541-9545.

(11) Willardson, B. M., Wilkins, J. F., Rand, T. A., Schupp, J. M., Hill, K. K., Keim, P., and Jackson, P. J. (1998) Development and testing of a bacterial biosensor for toluene-based environmental contaminants. Appl. Environ. Microbiol. 64, 1006-1012.

(12) Dueber, J. E., Wu, G. C., Malmirchegini, G. R., Moon, T. S., Petzold, C. J., Ullal, A. V., Prather, K. L. J., and Keasling, J. D. (2009) Synthetic protein scaffolds provide modular control over metabolic flux. Nat Biotech 27, 753-759.

(13) Steed, J. W., and Atwood, J. L. (2013) Supramolecular chemistry. John Wiley \& Sons.

(14) Stupp, S. I., and Palmer, L. C. (2014) Supramolecular chemistry and self-assembly in organic materials design. Chem. Mater. 26, 507-518.

(15) Yan, X., Wang, F., Zheng, B., and Huang, F. (2012) Stimuli-responsive supramolecular polymeric materials. Chem. Soc. Rev. 41, 6042-6065. 
(16) Uhlenheuer, D. A., Petkau, K., and Brunsveld, L. (2010) Combining supramolecular chemistry with biology. Chem. Soc. Rev. 39, 2817-2826.

(17) Brinkmann, J., Cavatorta, E., Sankaran, S., Schmidt, B., van Weerd, J., and Jonkheijm, P. (2014) About supramolecular systems for dynamically probing cells. Chem. Soc. Rev. 43, 44494469 .

(18) Boekhoven, J., and Stupp, S. I. (2014) 25th anniversary article: Supramolecular materials for regenerative medicine. Adv. Mater. 26, 1642-1659.

(19) Zaritsky, A., and Woldringh, C. L. (1978) Chromosome replication rate and cell shape in Escherichia coli: lack of coupling. J. Bacteriol. 135, 581-587.

(20) Darnton, N. C., Turner, L., Rojevsky, S., and Berg, H. C. (2007) On Torque and Tumbling in Swimming Escherichia coli. J. Bacteriol. 189, 1756-1764.

(21) Gross, L. (2006) Bacterial fimbriae designed to stay with the flow. PLoS Biol. 4, e314.

(22) Johan J M Pel and Ron van Mastrigt. (2007) Development of a CFD urethral model to study flow-generated vortices under different conditions of prostatic obstruction. Physiol. Meas. 28, 13.

(23) Niu, Y.-Y., and Chang, D.-Y. (2007) CFD simulation of shear stress and secondary flows in urethra. Biomed. Eng. Appl. Basis Commun. 19, 117-127.

(24) Nilsson, L. M., Thomas, W. E., Sokurenko, E. V., and V, V. (2006) Elevated shear stress protects Escherichia coli cells adhering to surfaces via catch bonds from detachment by soluble inhibitors. Appl. Environ. Microbiol. 72, 3005-3010.

(25) Thomas, W. E., Trintchina, E., Forero, M., Vogel, V., and Sokurenko, E. V. (2002) Bacterial adhesion to target cells enhanced by shear force. Cell 109, 913-923.

(26) Nilsson, L. M., Thomas, W. E., Trintchina, E., Vogel, V., and Sokurenko, E. V. (2006) Catch bond-mediated adhesion without a shear threshold - Trimannose versus monomannose interactions with the FimH adhesin of Escherichia coli. J. Biol. Chem. 281, 16656-16663.

(27) Roos, G., Wellens, A., Touaibia, M., Yamakawa, N., Geerlings, P., Roy, R., Wyns, L., and Bouckaert, J. (2013) Validation of reactivity descriptors to assess the aromatic stacking within the tyrosine gate of FimH. ACS Med. Chem. Lett. 4, 1085-1090.

(28) Crick, F. (1970) Central dogma of molecular biology. Nature 227, 561-563.

(29) Smith, A. (2008) Nucleic acids to amino acids: DNA specifies protein. Nat. Educ. 1, 126.

(30) Patthy, L. (1994) Introns and exons. Curr. Opin. Struct. Biol. 4, 383-392.

(31) Snyder, L., Peters, J. E., Henkin, T. M., and Champness, W. (2013) Molecular genetics of bacteria. American Society of Microbiology.

(32) Delidow, B. C., Lynch, J. P., Peluso, J. J., and White, B. A. (1993) Polymerase chain reaction, in PCR Protocols, pp 1-29. Springer.

(33) Rozhok, S., Shen, C. K.-F., Littler, P.-L. H., Fan, Z., Liu, C., Mirkin, C. A., and Holz, R. C. (2005) Methods for fabricating microarrays of motile bacteria. Small 1, 445-451.

(34) Hiratsuka, Y., Miyata, M., Tada, T., and Uyeda, T. Q. P. (2006) A microrotary motor powered by bacteria. Proc. Natl. Acad. Sci. 103, 13618-13623.

(35) Zhuang, J., Wright Carlsen, R., and Sitti, M. (2015) pH-taxis of biohybrid microsystems. Sci. Rep. 5 .

(36) Woolston, B. M., Edgar, S., and Stephanopoulos, G. (2013) Metabolic engineering: Past and future. Annu. Rev. Chem. Biomol. Eng. 4, 259-288.

(37) Kim, S., and Hahn, J.-S. (2014) Synthetic scaffold based on a cohesin-dockerin interaction for improved production of 2,3-butanediol in Saccharomyces cerevisiae. J. Biotechnol. 192, Part A, 192-196.

(38) Daugherty, P. S. (2007) Protein engineering with bacterial display. Membr. Eng. Des. 17, 474480. 
(39) Rice, J. J., and Daugherty, P. S. (2008) Directed evolution of a biterminal bacterial display scaffold enhances the display of diverse peptides. Protein Eng. Des. Sel. 21, 435-442.

(40) Getz, J. A., Rice, J. J., and Daugherty, P. S. (2011) Protease-resistant peptide ligands from a knottin scaffold library. ACS Chem. Biol. 6, 837-844.

(41) Getz, J. A., Cheneval, O., Craik, D. J., and Daugherty, P. S. (2013) Design of a cyclotide antagonist of neuropilin-1 and -2 that potently inhibits endothelial cell migration. ACS Chem. Biol. $8,1147-1154$.

(42) Henriques, S. T., Thorstholm, L., Huang, Y.-H., Getz, J. A., Daugherty, P. S., and Craik, D. J. (2013) A novel quantitative kinase assay using bacterial surface display and flow cytometry. PLoS ONE (Hirsch, E., Ed.) 8, e80474.

(43) Hall, S. S., and Daugherty, P. S. (2009) Quantitative specificity-based display library screening identifies determinants of antibody-epitope binding specificity. Protein Sci. 18, 1926-1934.

(44) Hyeon, J. E., Kim, S. W., Park, C., and Han, S. O. (2015) Efficient biological conversion of carbon monoxide ( $\mathrm{CO})$ to carbon dioxide $\left(\mathrm{CO}_{2}\right)$ and for utilization in bioplastic production by Ralstonia eutropha through the display of an enzyme complex on the cell surface. Chem. Commun. 51, 10202-10205.

(45) Xin, H., Li, Y., Liu, X., and Li, B. (2013) Escherichia coli-Based Biophotonic Waveguides. Nano Lett. 13, 3408-3413.

(46) Tsien, R. Y. (1998) The green fluorescent protein. Annu. Rev. Biochem. 67, 509-544.

(47) Ormö, M., Cubitt, A. B., Kallio, K., Gross, L. A., Tsien, R. Y., and Remington, S. J. (1996) Crystal structure of the Aequorea victoria green fluorescent protein. Science 273, 1392-1395.

(48) Prasher, D. C., Eckenrode, V. K., Ward, W. W., Prendergast, F. G., and Cormier, M. J. (1992) Primary structure of the Aequorea victoria green-fluorescent protein. Gene 111, 229-233.

(49) Tsien, R. Y. (2010) Nobel lecture: constructing and exploiting the fluorescent protein paintbox. Integr. Biol. 2, 77-93.

(50) Misteli, T., and Spector, D. L. (1997) Applications of the green fluorescent protein in cell biology and biotechnology. Nat Biotech 15, 961-964.

(51) Piston, D. W., and Kremers, G.-J. (2007) Fluorescent protein FRET: the good, the bad and the ugly. Trends Biochem. Sci. 32, 407-414.

(52) Nguyen, H. D., Dang, D. T., van Dongen, J. L. J., and Brunsveld, L. (2010) Protein dimerization induced by supramolecular interactions with cucurbit[8]uril. Angew. Chem. 122, 907-910.

(53) Smurthwaite, C. A., Hilton, B. J., O’Hanlon, R., Stolp, Z. D., Hancock, B. M., Abbadessa, D., Stotland, A., Sklar, L. A., and Wolkowicz, R. (2014) Fluorescent genetic barcoding in mammalian cells for enhanced multiplexing capabilities in flow cytometry. Cytometry A 85, 105-113.

(54) Wienken, C. J., Baaske, P., Rothbauer, U., Braun, D., and Duhr, S. (2010) Protein-binding assays in biological liquids using microscale thermophoresis. Nat. Commun. 1, 100.

(55) Hosse, R. J., Rothe, A., and Power, B. E. (2006) A new generation of protein display scaffolds for molecular recognition. Protein Sci. 15, 14-27.

(56) Gracy, J., and Chiche, L. (2011) Structure and modeling of knottins, a promising molecular scaffold for drug discovery. Curr. Pharm. Des. 17, 4337-4350.

(57) Moore, S. J., Leung, C. L., and Cochran, J. R. (2012) Knottins: disulfide-bonded therapeutic and diagnostic peptides. Pept. Modif. Pept. Drug Mol. 9, e3-e11.

(58) Lahti, J. L., Silverman, A. P., and Cochran, J. R. (2009) Interrogating and predicting tolerated sequence diversity in protein folds: application to E. elaterium trypsin inhibitor-II cystine-knot miniprotein. PLoS Comput. Biol. 5, e1000499. 
(59) Christmann, A., Walter, K., Wentzel, A., Krätzner, R., and Kolmar, H. (1999) The cystine knot of a squash-type protease inhibitor as a structural scaffold for Escherichia coli cell surface display of conformationally constrained peptides. Protein Eng. 12, 797-806.

(60) Gracy, J., Le-Nguyen, D., Gelly, J.-C., Kaas, Q., Heitz, A., and Chiche, L. (2008) KNOTTIN: the knottin or inhibitor cystine knot scaffold in 2007. Nucleic Acids Res. 36, D314-D319.

(61) Moore, S. J., Hayden Gephart, M. G., Bergen, J. M., Su, Y. S., Rayburn, H., Scott, M. P., and Cochran, J. R. (2013) Engineered knottin peptide enables noninvasive optical imaging of intracranial medulloblastoma. Proc. Natl. Acad. Sci. U.S.A. 110, 14598-14603.

(62) Heitz, A., Le-Nguyen, D., and Chiche, L. (1999) Min-21 and Min-23, the smallest peptides that fold like a cystine-stabilized $\beta$-sheet motif: design, solution structure, and thermal stability. Biochemistry (Mosc.) 38, 10615-10625.

(63) Souriau, C., Chiche, L., Irving, R., and Hudson, P. (2005) New binding specificities derived from Min-23, a small cystine-stabilized peptidic scaffold. Biochemistry (Mosc.) 44, 7143-7155.

(64) Chang, H.-J., Hsu, H.-J., Chang, C.-F., Peng, H.-P., Sun, Y.-K., Yu, H.-M., Shih, H.-C., Song, C.-Y., Lin, Y.-T., Chen, C.-C., Wang, C.-H., and Yang, A.-S. (2009) Molecular evolution of cystine-stabilized miniproteins as stable proteinaceous binders. Structure 17, 620-631.

(65) Jagadish, K., Borra, R., Lacey, V., Majumder, S., Shekhtman, A., Wang, L., and Camarero, J. A. (2013) Expression of fluorescent cyclotides using protein trans-splicing for easy monitoring of cyclotide-protein interactions. Angew. Chem. Int. Ed. 52, 3126-3131.

(66) Del Valle, E. M. M. (2004) Cyclodextrins and their uses: a review. Process Biochem. 39, 10331046.

(67) Easton, C. J., and Lincoln, S. F. (1999) Modified cyclodextrins. World Scientific Publishing Company.

(68) Eastburn, S. D., and Tao, B. Y. (1994) Applications of modified cyclodextrins. Biotechnol. Adv. $12,325-339$.

(69) Crini, G. (2014) Review: A history of cyclodextrins. Chem. Rev. 114, 10940-10975.

(70) Grancharov, G., Khosravi, E., Wood, D., Turton, A., and Kataky, R. (2005) Individually addressable recessed gold microelectrode arrays with monolayers of thio-cyclodextrin nanocavities. Analyst 130, 1351-1357.

(71) Masson, E., Ling, X., Joseph, R., Kyeremeh-Mensah, L., and Lu, X. (2012) Cucurbituril chemistry: a tale of supramolecular success. RSC Adv. 2, 1213-1247.

(72) Lee, J. W., Samal, S., Selvapalam, N., Kim, H.-J., and Kim, K. (2003) Cucurbituril homologues and derivatives: New opportunities in supramolecular chemistry. Acc. Chem. Res. 36, 621-630.

(73) Liu, S., Zavalij, P. Y., and Isaacs, L. (2005) Cucurbit[10]uril. J. Am. Chem. Soc. 127, 16798-16799.

(74) Kim, H., Heo, J., Jeon, W. S., Lee, E., Kim, J., Sakamoto, S., Yamaguchi, K., and Kim, K. (2001)

Selective inclusion of a hetero-guest pair in a molecular host: Formation of stable charge-transfer complexes in cucurbit [8] uril. Angew. Chem. Int. Ed. 40, 1526-1529.

(75) Rowland, M. J., Atgie, M., Hoogland, D., and Scherman, O. A. (2015) Preparation and supramolecular recognition of multivalent peptide-polysaccharide conjugates by cucurbit[8]uril in hydrogel formation. Biomacromolecules.16 (8), 2436-2448.

(76) An, Q., Brinkmann, J., Huskens, J., Krabbenborg, S., de Boer, J., and Jonkheijm, P. (2012) A supramolecular system for the electrochemically controlled release of cells. Angew. Chem. Int. Ed. 51, $12233-12237$.

(77) Sankaran, S., Kiren, M. C., and Jonkheijm, P. (2015) Incorporating bacteria as a living component in supramolecular self-assembled monolayers through dynamic nanoscale interactions. ACS Nano 9, 3579-3586. 
(78) Sindelar, V., Cejas, M. A., Raymo, F. M., Chen, W., Parker, S. E., and Kaifer, A. E. (2005) Supramolecular assembly of 2,7-dimethyldiazapyrenium and cucurbit[8]uril: a new fluorescent host for detection of catechol and dopamine. Chem. - Eur. J. 11, 7054-7059.

(79) Hu, C., Lan, Y., Tian, F., West, K. R., and Scherman, O. A. (2014) Facile method for preparing surface-mounted cucurbit[8]uril-based rotaxanes. Langmuir 30, 10926-10932.

(80) Wang, Y., Li, D., Wang, H., Chen, Y., Han, H., Jin, Q., and Ji, J. (2014) pH responsive supramolecular prodrug micelles based on cucurbit[8]uril for intracellular drug delivery. Chem. Commun. 50, 9390-9392.

(81) Murugavel, K. (2014) Benzylic viologen dendrimers: a review of their synthesis, properties and applications. Polym. Chem. 5, 5873-5884.

(82) Matsue, T., Kato, T., Akiba, U., and Osa, T. (1985) Inclusion, solubilization, and stabilization of two-electron reduced species of methyl viologen by cyclodextrins. Chem. Lett. 14, 1825-1828.

(83) Kim, H.-J., Jeon, W. S., Ko, Y. H., and Kim, K. (2002) Inclusion of methylviologen in cucurbit[7]uril. Proc. Natl. Acad. Sci. 99, 5007-5011.

(84) Jeon, W. S., Kim, H.-J., Lee, C., and Kim, K. (2002) Control of the stoichiometry in host-guest complexation by redox chemistry of guests: Inclusion of methylviologen in cucurbit[8]uril. Chem. Commun. 1828-1829.

(85) Tian, F., Jiao, D., Biedermann, F., and Scherman, O. A. (2012) Orthogonal switching of a single supramolecular complex. Nat. Commun. 3, 1207.

(86) Kang, J.-K., Hwang, I., Ko, Y. H., Jeon, W. S., Kim, H.-J., and Kim, K. (2008) Electrochemically controllable reversible formation of cucurbit[8]uril-stabilized charge-transfer complex on surface. Supramol. Chem. 20, 149-155.

(87) del Barrio, J., Horton, P. N., Lairez, D., Lloyd, G. O., Toprakcioglu, C., and Scherman, O. A. (2013) Photocontrol over cucurbit[8]uril complexes: stoichiometry and supramolecular polymers. J. Am. Chem. Soc. 135, 11760-11763.

(88) Stoffelen, C., Voskuhl, J., Jonkheijm, P., and Huskens, J. (2014) Dual stimuli-responsive selfassembled supramolecular nanoparticles. Angew. Chem. Int. Ed. 53, 3400-3404.

(89) Appel, E. A., Biedermann, F., Rauwald, U., Jones, S. T., Zayed, J. M., and Scherman, O. A. (2010) Supramolecular cross-linked networks via host-guest complexation with cucurbit[8]uril. J. Am. Chem. Soc. 132, 14251-14260.

(90) Beharry, A. A., and Woolley, G. A. (2011) Azobenzene photoswitches for biomolecules. Chem. Soc. Rev. 40, 4422-4437.

(91) Yamaguchi, H., Kobayashi, Y., Kobayashi, R., Takashima, Y., Hashidzume, A., and Harada, A. (2011) Photoswitchable gel assembly based on molecular recognition. Nat. Commun. 3, 603.

(92) Baroncini, M., Gao, C., Carboni, V., Credi, A., Previtera, E., Semeraro, M., Venturi, M., and Silvi, S. (2014) Light control of stoichiometry and motion in pseudorotaxanes comprising a cucurbit[7] uril wheel and an azobenzene-bipyridinium axle. Chem. - Eur. J. 20, 10737-10744.

(93) Wu, J., and Isaacs, L. (2009) Cucurbit[7]uril complexation drives thermal trans-cisazobenzene isomerization and enables colorimetric amine detection. Chem. - Eur. J. 15, 1167511680.

(94) Voskuhl, J., Sankaran, S., and Jonkheijm, P. (2014) Optical control over bioactive ligands at supramolecular surfaces. Chem. Commun. 50, 15144-15147.

(95) Norikane, Y., and Tamaoki, N. (2004) Light-driven molecular hinge: a new molecular machine showing a light-intensity-dependent photoresponse that utilizes the trans-cis isomerization of azobenzene. Org. Lett. 6, 2595-2598. 
(96) Horský, J., and Pitha, J. (1994) Inclusion complexes of proteins: Interaction of cyclodextrins with peptides containing aromatic amino acids studied by competitive spectrophotometry. $J$. Incl. Phenom. Mol. Recognit. Chem. 18, 291-300.

(97) Rekharsky, M. V., and Inoue, Y. (1998) Complexation thermodynamics of cyclodextrins. Chem. Rev. 98, 1875-1918.

(98) Linde, G. A., Junior, A. L., Faria, E. V. de, Colauto, N. B., Moraes, F. F. de, and Zanin, G. M. (2009) Taste modification of amino acids and protein hydrolysate by a-cyclodextrin. Food Res. Int. $42,814-818$.

(99) Tushar Satav. (2015, October 29) Creating biofunctionality by programming weak, noncovalent complexation between aromatic units of amino acids and macrocylic hosts. University of Twente.

(100) Buschmann, H.-J., Mutihac, L., Mutihac, R.-C., and Schollmeyer, E. (2005) Complexation behavior of cucurbit[6] uril with short polypeptides. Thermochim. Acta 430, 79-82.

(101) Lee, J. W., Lee, H. H. L., Ko, Y. H., Kim, K., and Kim, H. I. (2015) Deciphering the specific highaffinity binding of cucurbit[7]uril to amino acids in water. J. Phys. Chem. B 119, 4628-4636.

(102) Bush, M. E., Bouley, N. D., and Urbach, A. R. (2005) Charge-mediated recognition of nterminal tryptophan in aqueous solution by a synthetic host. J. Am. Chem. Soc. 127, 14511-14517.

(103) Heitmann, L. M., Taylor, A. B., Hart, P. J., and Urbach, A. R. (2006) Sequence-specific recognition and cooperative dimerization of $\mathrm{n}$-terminal aromatic peptides in aqueous solution by a synthetic host. J. Am. Chem. Soc. 128, 12574-12581.

(104) Sonzini, S., Ryan, S. T. J., and Scherman, O. A. (2013) Supramolecular dimerisation of middlechain Phe pentapeptides via $\mathrm{CB}[8]$ host-guest homoternary complex formation. Chem. Commun. 49, 8779-8781.

(105) Liu, Y., Yang, H., Wang, Z., and Zhang, X. (2013) Cucurbit[8]uril-based supramolecular polymers. Chem. - Asian J. 8, 1626-1632.

(106) Dang, D. T., Schill, J., and Brunsveld, L. (2012) Cucurbit[8]uril-mediated protein homotetramerization. Chem. Sci. 3, 2679-2684.

(107) Dang, D. T., Nguyen, H. D., Merkx, M., and Brunsveld, L. (2013) Supramolecular control of enzyme activity through cucurbit[8]uril-mediated dimerization. Angew. Chem. Int. Ed. 52, 29152919.

(108) Rowland, M. J., Appel, E. A., Coulston, R. J., and Scherman, O. A. (2013) Dynamically crosslinked materials via recognition of amino acids by cucurbit[8]uril. J. Mater. Chem. B 1, 29042910.

(109) Bosman, A. W., Sijbesma, R. P., and Meijer, E. . (2004) Supramolecular polymers at work. Mater. Today 7, 34-39.

(110) Stoffelen, C., and Huskens, J. (2013) Size-tunable supramolecular nanoparticles mediated by ternary cucurbit[8]uril host-guest interactions. Chem. Commun. 49, 6740-6742.

(111) Stoffelen, C., Voskuhl, J., Jonkheijm, P., and Huskens, J. (2014) Dual stimuli-responsive selfassembled supramolecular nanoparticles. Angew. Chem. Int. Ed. 53, 3400-3404.

(112) Dong, R., Pang, Y., Su, Y., and Zhu, X. (2015) Supramolecular hydrogels: synthesis, properties and their biomedical applications. Biomater. Sci. 3, 937-954.

(113) Tamesue, S., Takashima, Y., Yamaguchi, H., Shinkai, S., and Harada, A. (2010) Photoswitchable supramolecular hydrogels formed by cyclodextrins and azobenzene polymers. Angew. Chem. Int. Ed. 49, 7461-7464.

(114) Gong, Y.-H., Yang, J., Cao, F.-Y., Zhang, J., Cheng, H., Zhuo, R.-X., and Zhang, X.-Z. (2013) Photoresponsive smart template for reversible cell micropatterning. J. Mater. Chem. B 1, 20132017. 


\section{Chapter 2}

\section{A multivalent aromatic amino acid based peptide tag for supramolecular protein immobilization on $\beta$-cyclodextrin modified surfaces}

The immobilization of proteins on surfaces has gained major focus in the past decade due to its implication in application oriented and fundamental research. Microarrays, biosensors, implant coatings and drug delivery systems are examples of few applications for which protein immobilization on various types of surfaces has been explored. Surpamolecular host-guest systems have recently been explored to expand upon the versatility of such surfaces. Since binding occurs non-covalently in such systems, proteins can be immobilized in a dynamic, reversible and possibly even responsive manner. In this chapter, we have explored the possibility of developing a peptide tag that can be fused with proteins and enable them to bind B-cyclodextrin (B-CD) modified surfaces. The tag, named $Y_{4}$, consists of 4 weakly binding tyrosine residues appropriately spaced with glycine and serine residues (SGGYGGS) 4 to enable multivalent binding onto $6-\mathrm{CD}$ molecular printboards. We show that the peptide tag binds with an appreciable overall affinity of $14.6 \mu \mathrm{M}, 3$ orders better than the known monovalent affinity between tyrosine and $\mathrm{B}-\mathrm{CD}$ $(\sim 10 \mathrm{mM})$. The tag was also recombinantly fused to the $C$ terminal of a red fluorescent protein, TagRFP. The binding properties of this fusion protein, termed RFP-Y4, were probed using surface plasmon resonance (SPR), continuous flow microspotting and using B-CD silica nanoparticles. It was found that the $Y_{4}$ tag improves the adhesion of RFP towards $B-C D$ modified surfaces under conditions where non-specific adhesion is suppressed.

The project was done in collaboration with Dr. Qi An and Dr. Tushar N. Satav from the University of Twente 


\subsection{Introduction}

The possibility to immobilize active proteins on various types of surfaces has spearheaded the development of protein biochips, ${ }^{1,2}$ novel biosensors, ${ }^{3}$ nanoparticle based purification, ${ }^{4}$ delivery ${ }^{5}$ and detection techniques, ${ }^{4,6}$ etc. Various strategies have been employed to immobilize proteins either covalently or reversibly, ordered or randomly oriented and tightly or loosely packed depending upon the application. ${ }^{1,2}$ Non-specific, randomly oriented protein immobilization can be achieved by modifying surfaces with chemical groups that either cause physisorption of proteins or chemisorption by reacting with amino acid side groups on proteins such as amines, carboxyls and thiols. ${ }^{1,2}$ In most applications, specific and oriented adhesion is preferred and can be achieved by either chemically or genetically functionalizing the target protein with a motif that can bind to a modified surface. For example, biotinfunctionalized proteins can bind avidin-modified surfaces ${ }^{7}$ and His-tagged proteins can bind Ni-NTA modified surfaces. ${ }^{8}$ Additionally, to expand upon the applicability of such immobilized proteins, strategies to impart properties such as dynamism and responsiveness are being explored using supramolecular host-guest systems. ${ }^{9}$ Supramolecular chemistry deals with molecular components that interact through noncovalent forces. Supramolecular hosts such as crown ethers, calixarenes, cucurbiturils and cyclodextrins are usually macrocyclic molecules with cavities within which guest molecules with specific properties can bind. By careful selection of appropriate hosts and guests, various types of supramolecular architectures, like self-assembled monolayers, nanoparticles, vesicles and hydrogels, have been constructed. ${ }^{10}$ Since hostguest interactions are very similar to protein-ligand interactions, such architectures are currently being used to address issues in biological systems.

Amongst these, $\beta$-cyclodextrin ( $\beta-C D$ ) is one of the most extensively explored hosts for the construction of various biologically relevant supramolecular systems. ${ }^{11-13}$ It is a hollow truncated cone shaped molecule consisting of seven ( $a-1,4)$-linked $a-D$ glucopyranose units. It has a hydrophilic outer surface and a relatively hydrophobic cavity within which certain hydrophobic guest molecules can bind, for example ferrocene, adamantane, azobenzene and aromatic amino acids. ${ }^{14}$ The hydroxyl groups along the rims of $\beta-C D$ have been chemically modified to harbor various different types of functional groups. The functionalization of the narrow rim with long chain alkyl thioethers has been shown to enable the self-assembly of $\beta-C D$ into tightly packed monolayers on gold surfaces. These monolayers have been widely used as molecular printboards for application-oriented and fundamental studies..$^{15-21}$ Various other 
functionalities have also been used to enable $\beta$-CD to be immobilized on other types of surfaces such as glass, ${ }^{22}$ silver ${ }^{23}$ and graphene. ${ }^{24}$ Such surfaces have been used for the capture and detection of various biological entities. Variations of $\beta-C D$ have also been used with nanoparticles, polymers and vesicles indicating the extensive versatility of these host molecules. ${ }^{14}$

The usage of $\beta-C D$ molecular printboards for protein adhesion has been explored and described previously. ${ }^{15-17,20}$ Early attempts utilized avidin-biotin ${ }^{15-17}$ or Ni-NTA ${ }^{17}$ as an intermediary layer to which biotin-labeled or His-tagged proteins were able to adhere respectively. Gonzalez-Campo et al. further showed that biotinylated alkaline phosphatase immobilized on $\beta$-CD surfaces through an intermediary streptavidin-biotin layer still exhibits appreciable enzymatic activity. ${ }^{20} \mathrm{~A}$ direct approach for site-specifically immobilizing proteins onto $\beta-C D$ monolayers was explored by Yang et al. ${ }^{21}$ A yellow fluorescent protein was labeled with ferrocene (YFP-FC), which allowed the protein to specifically bind $\beta-C D$ in an oriented manner and also enabled the controlled release of the protein from the surface by electrochemical reduction of ferrocene. They also showed that dimerized versions of the YFP-Fc, which were able to bind the $\beta-C D$ monolayers through two ferrocene groups simultaneously, had a higher affinity compared to monovalently binding monomeric YFP-Fc. However this technique still requires chemical modification and purification of the proteins. A favorable modification to the system would be to identify the possibility of immobilizing proteins without the requirement of chemically modifying them. Among the numerous hydrophobic molecules which bind to $\beta-C D$, the aromatic amino acids such as tyrosine, show very weak binding with dissociation constant of ca. 1-100 mM.25,26 Peptide tags with multiple copies of theses aromatic amino acids could potentially adhere in a multivalent manner with $\beta$-CD-modified surfaces. Such a tag could be genetically fused with any protein of interest and recombinantly produced using a bacterial expression system. These engineered proteins could then bind $\beta-C D$-modified surfaces directly from bacterial cell lysates.

In this chapter, we have tested the possibility of developing a peptide tag containing four tyrosine residues $\left(\mathrm{Y}_{4}\right)$ appropriately spaced to be able to adhere to $\beta-C D$ monolayers in a multivalent manner. Tyrosine has been shown to bind $\beta-C D$ in a monovalent manner with a very low affinity. We first showed that our peptide tag adheres to $\beta-C D$ molecular printboards with a significantly higher affinity. The peptide tag was then fused to the C-terminal of a red fluorescent protein (RFP). The binding of such a fusion protein was then studied on $\beta-C D$ monolayers on gold, glass and silica nanoparticles. Improved binding of the peptide tag-fused RFP was observed in all cases and 
possibilities of protein separation from cell lysates was also tested. The general schematic is presented in Figure 2.1.

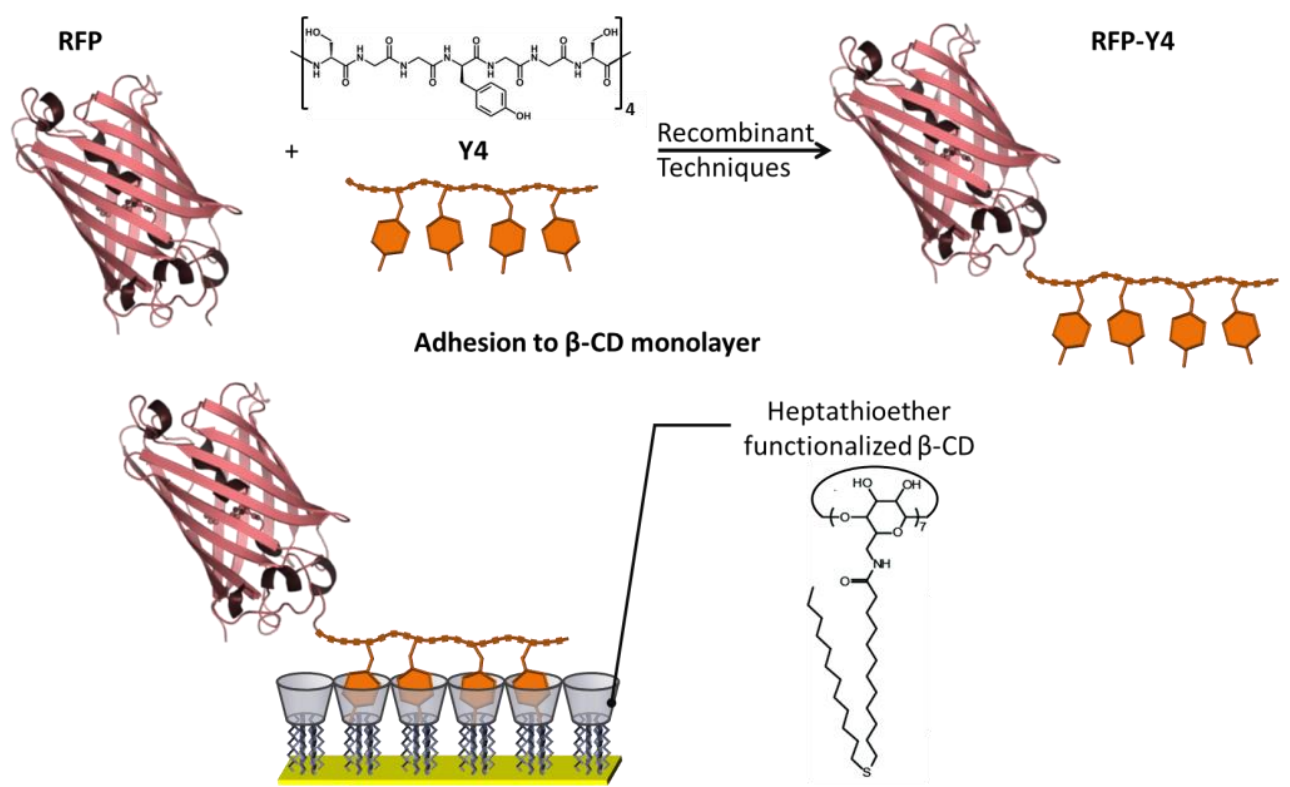

Figure 2.1. RFP is fused with the multivalent peptide tag, $Y_{4}$, using recombinant techniques and the adhesion of this fusion protein (RFP-Y4) has been studied on B-CD monolayers.

\subsection{Results and Discussions}

\subsubsection{Peptide tag}

Tyrosine containing peptides have been shown to weakly bind $\beta-C D$ with low millimolar affinities $\left(K_{d} \sim 10 \mathrm{mM}\right) \cdot{ }^{27}$ A peptide tag with multiple tyrosine residues could be expected to bind tightly-packed $\beta-C D$ SAMs with an appreciable affinity due to multivalent interactions. In $\beta-C D$ molecular printboards on gold, the center-to-center spacing of adjacent $\beta-C D$ cavities was previously found to be $2.1 \mathrm{~nm}$. Hence, a peptide with the structural motif $\mathrm{Ac}(\mathrm{SGGYGGS})_{4}$, denoted as $\mathrm{Y}_{4}$ from now, was synthesized with about $2.7 \mathrm{~nm}$ maximum separation between adjacent $Y$ residues. This distance between adjacent $Y$ residues permits their binding with $\beta-C D$ receptors in the SAMs as shown in the schematic illustration of the molecular structures of the $\gamma_{4}$ peptide and $\beta$ CD SAM (Figure 2.2a). 
The affinity between the $Y_{4}$ peptide tag and $\beta$-CD SAMs on gold was determined by a surface plasmon resonance (SPR) titration experiment. Different concentrations of buffered solutions of the $Y_{4}$ peptide were flowed over the $\beta-C D$ SAM surface. Adhesion of the $\mathrm{Y}_{4}$ peptide to the surface results in a change in the coupling angle directly proportional to the surface coverage of the guest. As shown in Figure $2.2 \mathrm{~b}$, various concentrations of the $Y 4$ peptide were flowed through the SPR fluidic chamber and the change in coupling angle was recorded. After each concentration, the surfaces were regenerated for a next injection by washing off the peptides using a $5 \mathrm{mM} \beta$-CD solution. Plotting the maximum change in coupling angle against the corresponding concentration of the $Y_{4}$ peptide provided a binding curve as shown in Figure $2.2 \mathrm{c}$. This was fitted with the Langmuir equation, which provided a dissociation constant of 14.6 $\mu M$. Thus the overall binding affinity of the multivalent $Y_{4}$ peptide tag was almost 3 orders of magnitude better than the monovalent interaction of $Y$ with $\beta-C D(\sim 10 \mathrm{mM})$. To ensure that the amino acids $S$ and $G$ do not give rise to non-specific interactions, the peptide $\mathrm{Ac}(\mathrm{SGGSGGS})_{5}$ was synthesized, where the $\mathrm{Y}$ residues were replaced by $\mathrm{S}$ residues. In this case five repeats of the sequence were included instead of four to ensure a slightly higher molecular weight $(2.507 \mathrm{kDa})$ compared to the $\mathrm{Y}_{4}$ peptide $(2.322 \mathrm{kDa})$. An SPR titration experiment similar to the one in Figure 2.3a was performed and it was found that only negligible responses were recorded at each concentration (Figure 2.2d). Even at the highest concentration of $300 \mu \mathrm{M}$, the overall change in coupling angle was less than what was observed with $2 \mu \mathrm{M}$ of the $\mathrm{Y}_{4}$ peptide. This result indicated that binding of the $Y_{4}$ peptide occurred only due to the $Y$ residue, and the spacers had a negligible contribution to the overall affinity of the peptide with the $\beta$-CD SAM. 
a)
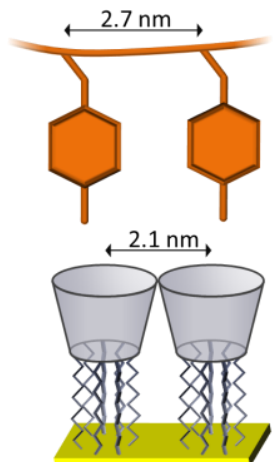

c)

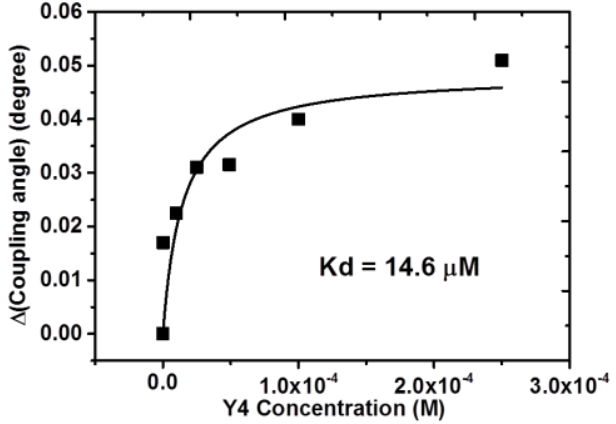

b)

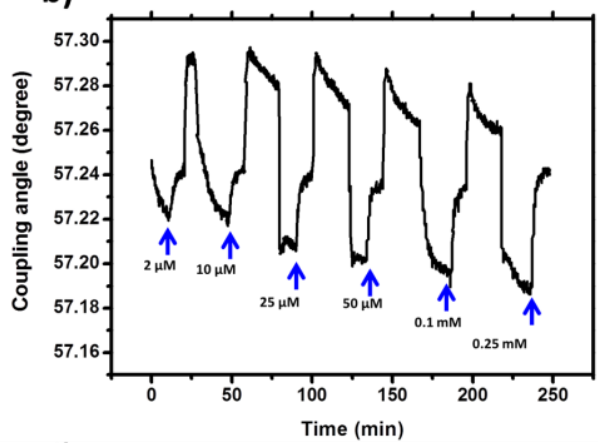

d)

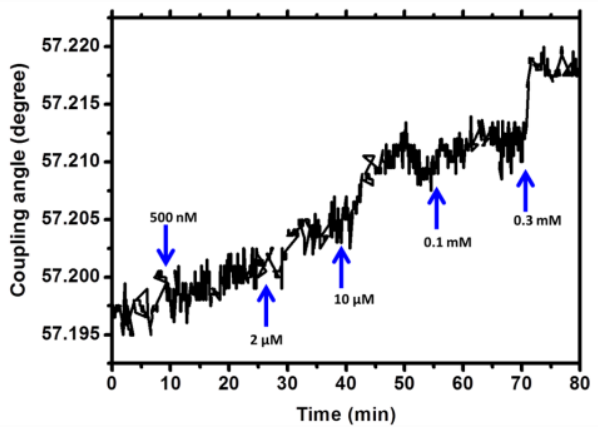

Figure 2.2. a) Distance between the centres of tightly packed B-CD cavities compared to maximum distance between adjacent tyrosine residues in the Y4 peptide. b) SPR titration following the binding of different concentrations of the $Y_{4}$ peptide on $6-C D$ molecular printboards on gold. $c$ ) Maximum $S P R$ responses plotted against corresponding $Y_{4}$ concentrations and fitted with the Langmuir equation (solid line). d) Control SPR titration of (SGGSGGS) $)_{5}$ peptide on on B-CD molecular printboards on gold.

\subsubsection{Recombinant protein}

In order to analyse the effect of the $Y_{4}$ peptide tag on the binding of proteins to $\beta-C D$ SAMs, we genetically fused the tag to a red fluorescent protein, TagRFP. TagRFP is a monomeric fluorescent protein known for its fast maturation, remarkable brightness, prolonged fluorescence lifetime, reduced photobleaching and high pH stability. ${ }^{28}$ It is also known to be an ideal fluorescent protein for recombinant expression in E.coli, with other proteins or peptides genetically fused to it. So, we genetically fused the $\mathrm{Y}_{4}$ peptide tag to the C-terminal of TagRFP. The genetic sequence of this fusion protein (RFP-Y4) was sequenced and found to be correct (see in Experimental section). The 
fusion protein also had an N-terminal hexa-His tag, which, after expression in E. coli, was used to purify the protein using a nickel affinity column. As a control, unmodified wild-type TagRFP (RFP) was used.

\subsubsection{SPR analysis}

SPR titrations were performed with different concentrations of RFP (red) and RFP-Y4 (black) on $\beta-C D$ molecular printboards (Figure 2.3a). RFP-Y4 produced significant SPR responses even at concentrations as low as $5 \mathrm{nM}$. At concentrations above $50 \mathrm{nM}$, RFP also produces significant SPR responses, though lower than the responses produced by RFP-Y4, indicating that non-specific adhesion occurred. This is probably due to the presence of other aromatic amino acids on its surface as shown in Figure $2.3 \mathrm{~b}$ (blue $=\mathrm{Y}$, green $=F$, yellow $=W$ ). In most studies so far, Tween-20 is used to supress these nonspecific interactions since it can mask the hydrophobic groups. ${ }^{29}$ However in our case, Tween-20 would also affect the binding strength of the $\mathrm{Y}_{4}$ tag and we wanted to observe the effect of the tag without any external disturbances. From these SPR response curves, the binding curve of Figure $2.3 \mathrm{C}$ was obtained by plotting the maximum response values against corresponding protein concentrations. By fitting these plots using a Langmuir equation, overall dissociation constants $\left(K_{d}\right)$ were obtained. Non-specific adhesion of RFP seems to occur with a $K_{d}$ value of $281 \mathrm{nM}$ but RFP-Y 4 has a dissociation constant that is 1 order lower $\left(K_{d}=21 n M\right)$, clearly indicating that the presence of the $Y_{4}$ tag improves the binding affinity of the protein with the $\beta$ $C D$ modified surface. Significant differences in surface adhesion are seen at very low concentrations below $20 \mathrm{nM}$ as shown in Figure 2.3d. At these concentrations, while RFP-Y4 binds to a considerable extent, nearly no binding of RFP was observed. These results indicate that the $\mathrm{Y}_{4}$ tag improves the ability of protein binding on $\beta-C D$ surfaces and strategies to reduce non-specific adhesion are required. 
a)

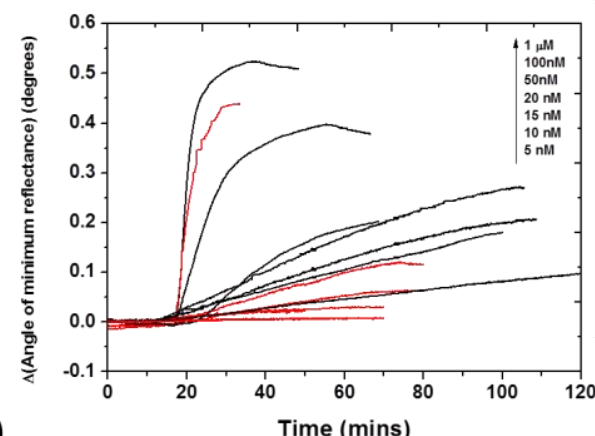

c)

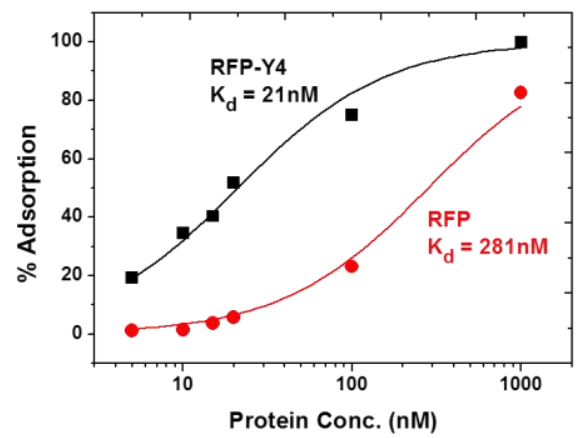

b)
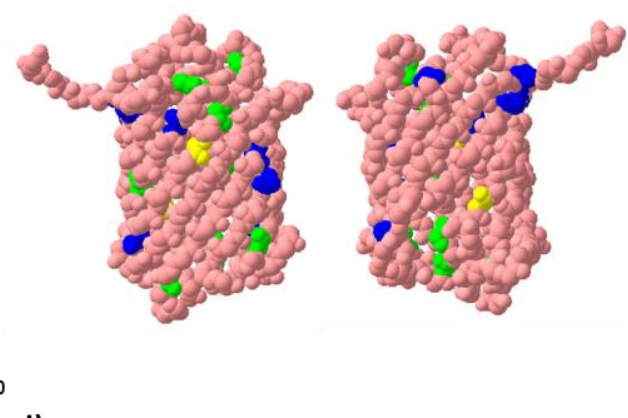

d)

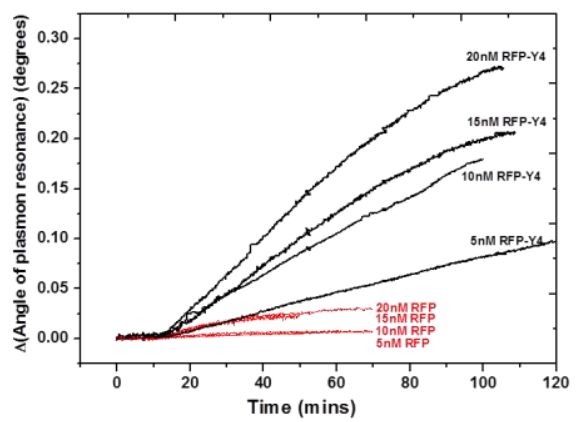

Figure 2.3. a) SPR titration response curves of RFP-Y4 (black) and RFP (red) on 6-CD molecular printboards. b) 3D structures of TagRFP ( $180^{\circ}$ rotated around Z-axis, PDB ID: 3 T6H $^{30}$ with aromatic amino acids highlighted (blue $=Y$, green $=F$, yellow $=W$ ). $c$ ) Binding curves of RFP-Y4 (black) and RFP (red) obtained by plotting maximum SPR responses against corresponding concentrations. Solid lines represent Langmuir equation (eq 2.1) fitting. d) SPR responses by RFP-Y4 (black) and RFP (red) at low concentrations $<=20 \mathrm{nM}$.

\subsubsection{Continuous flow micro-spotting}

To test conditions for selective adhesion of the RFP-Y4 on $\beta-C D$ surfaces, continuous flow micro-spotting of the proteins was done on $\beta-C D$ modified glass surfaces. RFP-Y 4 and RFP solutions at different concentrations were simultaneously flowed over separate spots on $\beta-C D$ modified glass surfaces for an hour. After a brief wash with water, the spots were imaged using an epifluorescent microsope. When conditions were maintained similar to the SPR measurements, (without BSA), it was seen that both proteins adhere to the surface in a similar manner at all concentrations (Figure 2.4 a row 1 ). Next, $1 \%$ bovine serum albumin (BSA) was added to the protein solutions as a blocking agent in an attempt to reduce non-specific adhesion. When this was done, 38 
even though the overall fluorescence intensities seemed to drop, clear differences in surface adhesion of the two proteins were observed as shown in Figure 2.4a, row 2. The fluorescent intensities of these spots were plotted against corresponding protein concentrations as shown in Figure $2.4 \mathrm{~b}$ and the resulting sigmoidal curves were fitted with the Langmuir equation. The obtained $K_{d}$ values indicate that the concentration at which half-maximal binding occurred was similar for both RFP-Y4 and RFP, but the amount of adhered RFP-Y 4 was significantly higher than that of RFP even at $1000 \mathrm{nM}$. This indicated that non-specific adhesion could be reduced by optimizing solution parameters. To test the reversibility of this binding, the surfaces were first sonicated in PBS and then rinsed with PBS + Tween-20. PBS sonication did not seem to be able to remove the proteins from the surface however washing with PBS and Tween-20 almost all traces of the proteins. Due to the high affinity between the RFP-Y4 protein and the $\beta-C D$ monolayer, even sonication with PBS was insufficient to remove it from the surface. However when Tween-20 was included in the buffer, it would have effectively masked the aromatic amino acids on the protein surface, preventing their binding to $\beta$ CD. This further proves that surface adhesion occurred through hydrophobic interactions between the aromatic amino acids and the cavity of $\beta-C D$. 


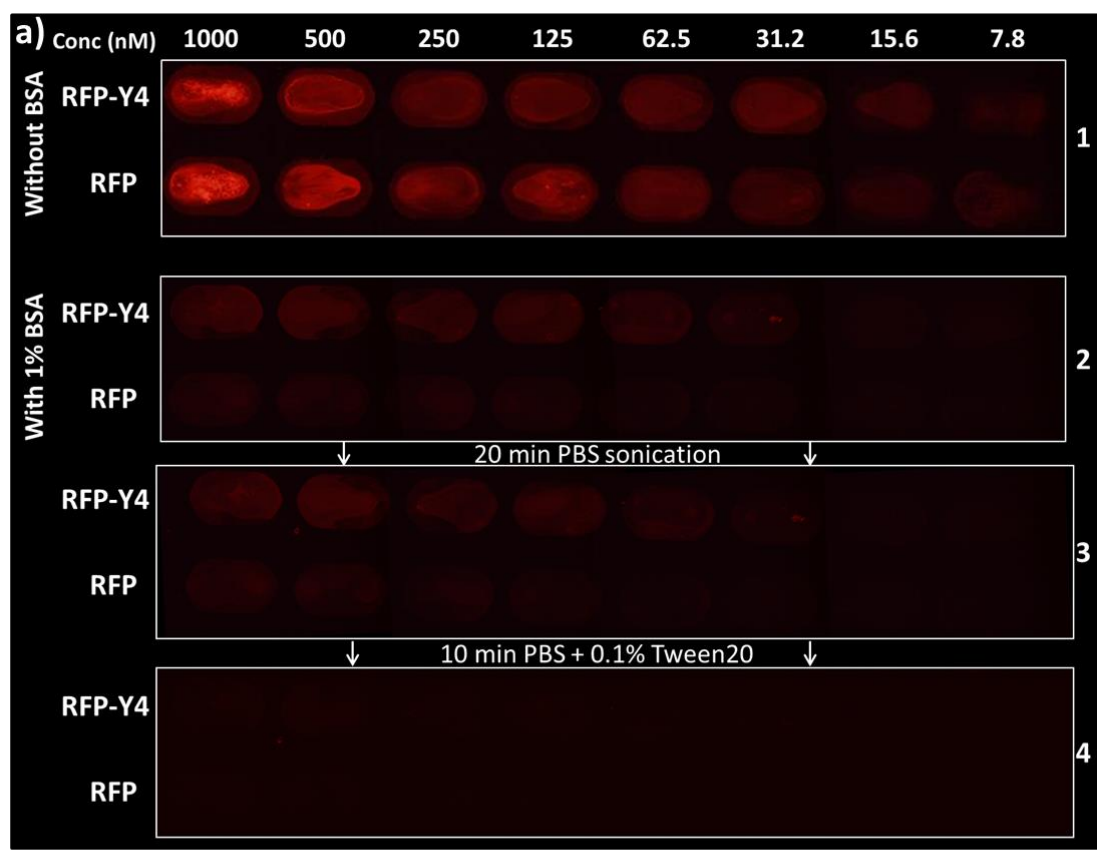

b)

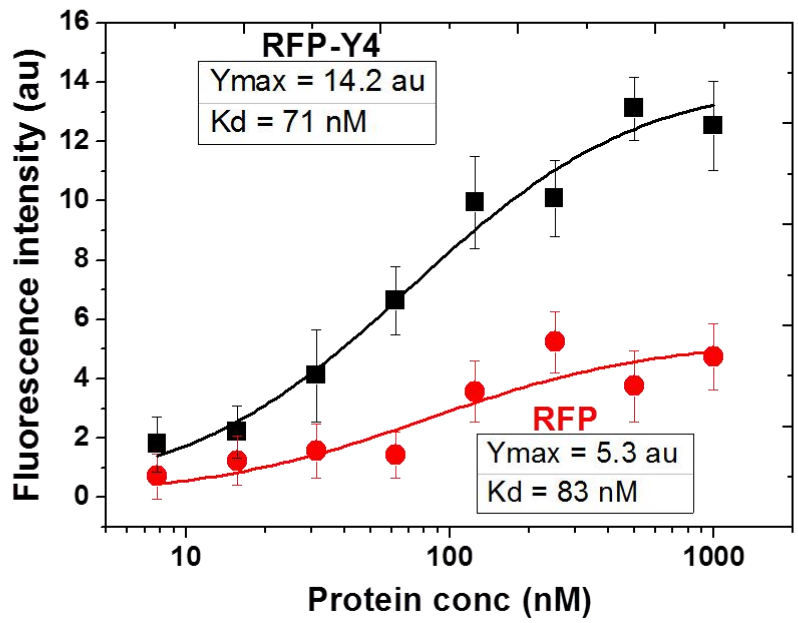

Figure 2.4. a) Fluorescent images of $8-C D$ monolayers on glass spotted with different concentrations of RFP-Y 4 and RFP either without or with BSA followed by two different wash procedures. b) Fluorescent intensities of the RFP-Y4 (black) and RFP (red) spots plotted against corresponding concentrations. Solid lines represent fitted curves using Langmuir equation. Data presented as mean \pm STD, $n=2$. 


\subsubsection{6-CD silica nanoparticles}

Next we tested the adsorption of the proteins onto silica nanoparticles covered with $\beta$ $\mathrm{CD}$ ( $\beta-\mathrm{CD} \mathrm{SiO}_{2} \mathrm{NPs}$ ) (Figure 2.5a). After trying out various parameters such as nanoparticle concentration, incubation time, centrifugation speeds and washing procedure, a relatively optimized protocol was identified. In short, the proteins were incubated with $0.025 \% \mathrm{wt} / \mathrm{vol} \beta-\mathrm{CD} \mathrm{SiO}{ }_{2} \mathrm{NPs}$ for 30 mins then the NPs were separated by centrifugation, rinsed and finally resuspended in PBS. Fluorescence intensities of the NP solutions were determined from images obtained using an epifluorescent microsope (Figure 5b). We observed that at all concentrations, the RFP-Y4 nanoparticles showed higher fluorescence intensities compared to the nanoparticles mixed with RFP (Figure 2.5b). As expected, non-specific adhesion increased with increasing concentration of RFP. On the contrary, beyond $100 \mathrm{nM}, \mathrm{RFP}-\mathrm{Y}_{4}$ seemed to saturate. These trends are reflected in the RFP-Y4:RFP fluorescence intensity ratios, where higher ratios are seen at $25 \mathrm{nM}$ and $50 \mathrm{nM}$ concentrations. We then tested the possibility of using such nanoparticles to isolate RFP-Y4 from the presence of cell lysate proteins. Different concentrations of RFP and RFP-Y4 were mixed with solutions of freshly prepared cell lysate protein solutions and nanoparticles were added and isolated as already explained (Figure 2.5C). At the lowest concentration of $25 \mathrm{nM}$, the binding of RFP and RFP-Y4 seemed to be almost completely blocked. Interestingly, at $50 \mathrm{nM}$ a transition was seen where significant adhesion of RFP-Y4 occurred while nonspecific adhesion of RFP was considerably reduced as can be seen from the RFP-Y4:RFP ratio. Above $50 \mathrm{nM}$, the amount of RFP and RFP-Y 4 bound to the nanoparticles seemed to increase with increasing concentrations even though at least twice as much RFP-Y4 seemed to be bound compared to RFP. These results indicate that such a peptide tag could potentially be used to isolate recombinant proteins from cell lysates. However further investigations are required to optimize the selectivity for example by adding BSA, Tween-20, changing buffer properties, varying nanoparticle concentrations etc. 

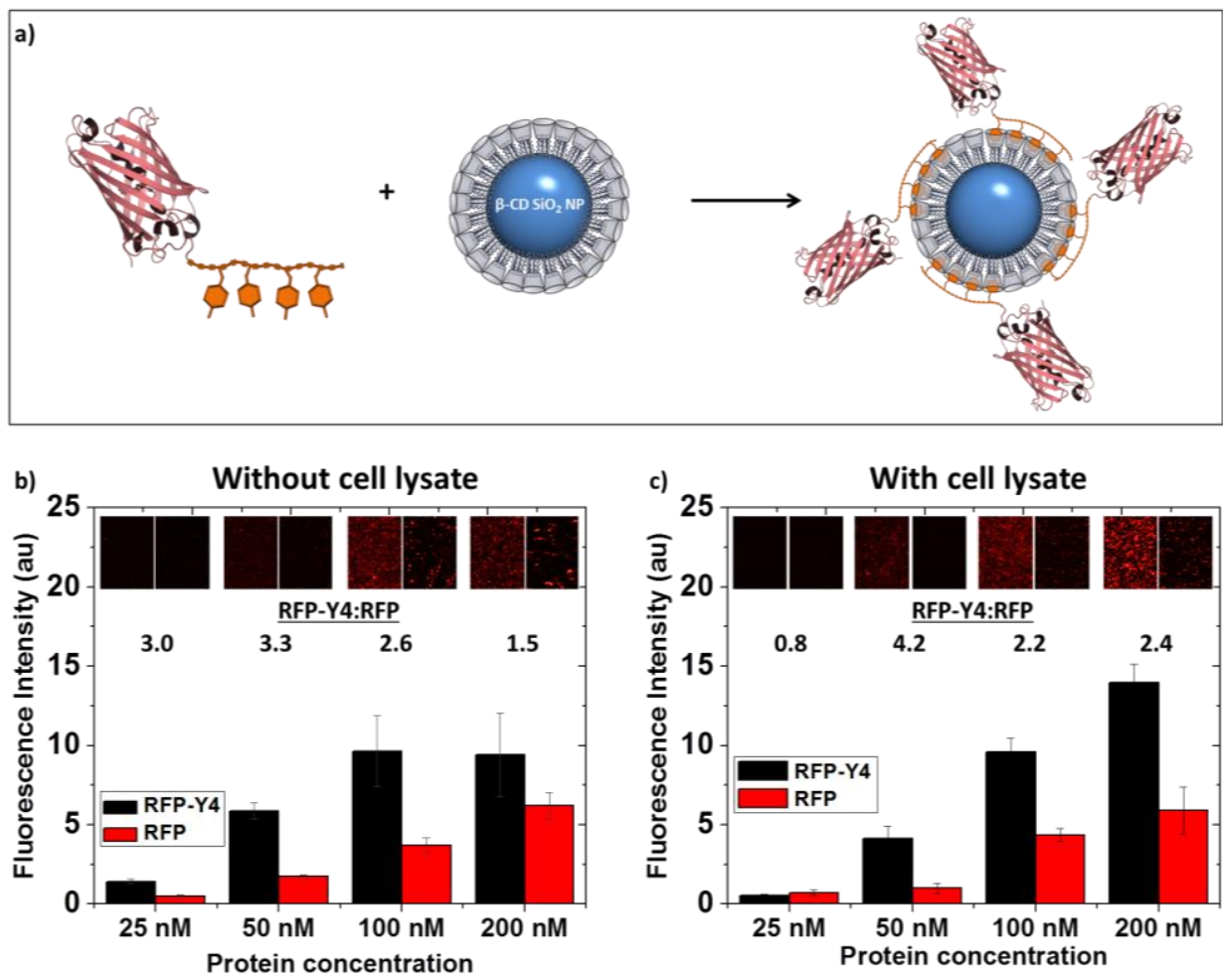

Figure 2.5. a) General schematic of RFP-Y 4 binding $6-C D \mathrm{SiO}_{2} \mathrm{NPs}$. Column plots of fluorescence intensities of RFP-Y4 (black) and RFP (red) bound to $\mathrm{B}-\mathrm{CD} \mathrm{SiO}_{2} \mathrm{NPs} b$ ) without cell lysate and c) with cell lysate. Representative fluorescent images and RFP-Y4:RFP fluorescence intensity ratios corresponding to each sample are provided within the plots. Width of the fluorescent images correspond to $200 \mu \mathrm{m}$. Data is presented as mean \pm STE, $n=4$.

\subsection{Conclusion}

In summary, we were able to validate with SPR, continuous flow microspotting and using NPs that the $\mathrm{Y}_{4}$ tag improves the binding of RFP to $\beta-C D$ surfaces. One major hurdle faced was non-specific adhesion of RFP, which has been partially addressed by identifying that at low concentrations non-specific adhesion were considerably reduced and also that BSA can be used as a blocking agent. Further work needs to be done to optimize the selectivity of the systems. Longer tags with more SGGYGGS repeats ( $Y_{5}$, Y6.. etc.) might help improve the system by allowing much higher specific binding at very low concentrations. Buffer properties, detergents such as Tween-20, blocking 
agents such as BSA, pH and temperature effects also need to be extensively studied to determine the best conditions for specific adhesion. Once these parameters are optimized, such a tag could potentially be used for protein purification, dynamic presentation on surfaces, drug delivery, etc.

\subsection{Acknowledgements}

We would like to thank Dr. Qi An and Tushar Satav for the production, characterization and analysis of the $\mathrm{Y}_{4}$ peptide. We would like to thank Carlo Nicosia for generously providing well characterized $\beta-\mathrm{CD} \mathrm{SiO}{ }_{2} \mathrm{NPs}$. We would also like to thank Dr. Dorothee Wasserberg for providing the TagRFP sequence containing plasmids.

\subsection{Experimental Section}

Equipment. Polymerase Chain Reaction (PCR) was performed using a Peqlab Primus 25 advanced thermocycler. Fast Protein Liquid Chromatography (FPLC) was performed using ÄKTApurifier (GE Healthcare Life Sciences) in combination with a Frac-950 fractionation collector (GE Healthcare Life Sciences). The used column was a Superdex 75 10/300GL (GE Healthcare Life Sciences) and had a separation range for molecules with molecular weights between 3000 and 70000 Da. UV-Vis measurements to determine DNA and protein concentrations were performed using a Thermo Scientific Nanodrop 1000. Surface plasmon resonance (SPR) experiments were conducted using $50 \mathrm{~nm}$ SPR gold substrates from Ssens bv on a Resonant-probes SPR. The reflectivity was measured at fixed angle at which point the linear region of the SPR curve stopped. Surface adhesion measurements were carried out under conditions of constant flow (100 $\mu \mathrm{L} / \mathrm{min}$ ) using a pH 7.4 buffer of PBS. An Olympus microscope 1 X71 with filters were used for recording fluorescent images. Continuous flow microspotting was performed with a 48 channel PDMS CFM device from Wasatch microfluidics LLC. Spotting was performed at $150 \mu \mathrm{L} / \mathrm{min}$ back and forth flow for an hour.

Peptide synthesis. The Ac(SGGYGGS) $)_{4}$ and $A c(S G G S G G S)_{5}$ peptides were synthesized with solid phase peptide synthesis using Fmoc-Rink Amide MBHA resin, following standard Fmoc protocols. Purification of the peptides were done with reversed phase HPLC. Preparative column (Waters 2535 quaternary gradient module with XBridgeTM prep C18 $5 \mu \mathrm{m}$ OPDTM $19 \mathrm{X} 250 \mathrm{~mm}$ preparative column) with eluents $0.1 \%$ aqueous TFA and $0.1 \%$ TFA in acetonitrile to purify the crude peptides. Extent of purification was checked using an analytical HPLC (Waters 2535 quaternary gradient module XBridge C18 $5 \mu \mathrm{m}$ column with $4.6 \times 250 \mathrm{~mm}$ dimensions). Same eluents as that of preparative 
HPLC were used. HPLC retention times were observed following analytical HPLC with a solvent gradient of $0-90 \%$ acetonitrile over $90 \mathrm{~min}$ timescale. Mass of purified peaks were determined using LC-MS (Waters 2535 module coupled to micromass LCT). The quality of the peptides were checked by analytical HPLC and ESI Mass spectra.

Peptide $\mathrm{Ac}(\mathrm{SGGYGGS})_{4}$ was synthesized and purified, with a yield of $28 \mathrm{mg}, 12 \%$. ESI mass spectra of peptide $\mathrm{Ac}(\mathrm{SGGYGGS})_{4}$ confirmed the successful synthesis of the peptide. Chemical Formula: $\mathrm{C}_{94} \mathrm{H}_{129} \mathrm{~N}_{29} \mathrm{O}_{41}, \mathrm{MW}: 2319.89 \mathrm{~g} / \mathrm{mol}$. $\left[\mathrm{M}+2 \mathrm{H}^{+}\right] / 2$ : calculated: 1161.0; found: 1161.3; $\left[\mathrm{M}+3 \mathrm{H}^{+}\right] / 3$ : calculated: 773.3; found: 774.1. The analytical HPLC showed a single peak, indicating product of high purity.

Peptide $\mathrm{AC}(\mathrm{SGGSGGS})_{5}$ was synthesized and purified, with a yield of $6 \%$. ESI mass spectra of peptide $A c(S G G S G G S)_{5}$ confirmed the successful synthesis of the peptide. Chemical Formula: $\mathrm{C}_{87} \mathrm{H}_{140} \mathrm{~N}_{36} \mathrm{O}_{51}$, Exact mass: $2504.95 \mathrm{~g} / \mathrm{mol}$. $\left[\mathrm{M}+\mathrm{Na}^{+}+\mathrm{H}^{+}\right] / 2$ : calculated 1264.5, found: 1264.2; [M+2 $\left.\mathrm{H}^{+}\right] / 2$ : calculated: 1253.5 , found: 1253.1. The analytical HPLC showed a single peak, indicating product of high purity.

Molecular cloning of RFP-Y4. pRSETB TagRFP vector was obtained from Dorothee Wasserberg. ssDNA sequences corresponding to the $\mathrm{Y}_{4}$ peptide and primers to extend the $\mathrm{N}$ and $\mathrm{C}$ termini with the restriction sites 5'-EcoRI and 3'-HindIII were ordered from Eurofins MWG Operon, Germany. Amplification of the extended genetic construct was performed by PCR using pfu polymerase. The final genetic construct was digested with appropriate restriction enzymes and inserted it at the 3 ' end of the TagRFP sequence in the plasmid using T4 DNA ligase (New England Biolabs inc.). The resulting pRESETB TagRFP-Y4 plasmid was transformed into NovaBlue ultracompetent cells (Novagen) and grown overnight on LB agar plates containing $100 \mathrm{mg} / \mathrm{L}$ ampicillin. Plasmids were extracted from individual colonies using Qiagen spin miniprep kit and the sequenced by Eurofins MWG Operon, Germany using a standard T7 terminal reverse primer. The plasmid was then transformed into the expression host, BL21DE3 pLysS competent cells (Novagen) and grown overnight on LB agar plates containing $34 \mathrm{mg} / \mathrm{L}$ chloramphenicol and 100mg/L ampicillin. Individual colonies were grown in LB media containing the mentioned antibiotics. For long term storage, $15 \%$-glycerol bacterial stocks were made and placed at $-80^{\circ} \mathrm{C}$. 
RFP-Y4 DNA sequence:

ATGCGGGGTTCTCATCATCATCATCATCATGGTATGGCTAGCATGACTGGTGGACAGCAAATGG GTCGGGATCTGTACGACGATGACGATAAGGATCCGATGAGCGAGCTGATTAAGGAGAACATG CACATGAAGCTGTACATGGAGGGCACCGTGAACAACCACCACTTCAAGTGCACATCCGAGGGC GAAGGCAAGCCCTACGAGGGCACCCAGACCATGAGAATCAAGGTGGTCGAGGGCGGCCCTCT CCCCTTCGCCTTCGACATCCTGGCTACCAGCTTCATGTACGGCAGCAGAACCTTCATCAACCAC ACCCAGGGCATCCCCGACTTCTTTAAGCAGTCCTTCCCTGAGGGCTTCACATGGGAGAGAGTCA CCACATACGAAGACGGGGGCGTGCTGACCGCTACCCAGGACACCAGCCTCCAGGACGGCTGCC TCATCTACAACGTCAAGATCAGAGGGGTGAACTTCCCATCCAACGGCCCTGTGATGCAGAAGA AAACACTCGGCTGGGAGGCCAACACCGAGATGCTGTACCCCGCTGACGGCGGCCTGGAAGGCA GAAGCGACATGGCCCTGAAGCTCGTGGGCGGGGGCCACCTGATCTGCAACTTCAAGACCACAT ACAGATCCAAGAAACCCGCTAAGAACCTCAAGATGCCCGGCGTCTACTATGTGGACCACAGAC TGGAAAGAATCAAGGAGGCCGACAAAGAGACCTACGTCGAGCAGCACGAGGTGGCTGTGGCC AGATACTGCGACCTCCCTAGCAAACTGGGGCACAAGGAATTCAGCGGAGGATATGGAGGAAG CAGCGGAGGATATGGAGGAAGCAGCGGAGGATATGGAGGAAGCAGCGGAGGATATGGAGGA AGCGAAGCTTGA

RFP-Y4 protein sequence:

MRGSHHHHHHGMASMTGGQQMGRDLYDDDDKDPMSELIKENMHMKLYMEGTVNNHHFK CTSEGEGKPYEGTQTMRIKVVEGGPLPFAFDILATSFMYGSRTFINHTQGIPDFFKQSFPEGFTWE RVTTYEDGGVLTATQDTSLQDGCLIYNVKIRGVNFPSNGPVMQKKTLGWEANTEMLYPADGGL EGRSDMALKLVGGGHLICNFKTTYRSKKPAKNLKMPGVYYVDHRLERIKEADKETYVEQHEVAV ARYCDLPSKLGHKEFSGGYGGSSGGYGGSSGGYGGSSGGYGGSEA

Blue = Hexa-His Tag

Red $=$ TagRFP

Orange $=\mathrm{Y}_{4}$ peptide tag

Expression and purification of RFP and RFP-Y4. $5 \mathrm{ml}$ bacterial starter cultures were grown overnight from glycerol stocks at $37^{\circ} \mathrm{C}$ with shaking in LB media containing appropriate antibiotics. This was then transferred into $1 \mathrm{~L}$ of the same media and the cultures were grown till they attained O.D.60onm values between 0.4 and 0.8 . Protein expression was then induced using isopropyl-ß-D-1-thiogalactopyranoside (IPTG) at a final concentration of $0.1 \mathrm{mM}$. These cultures were grown overnight at $18^{\circ} \mathrm{C}$ with shaking. The cultures were then spun down at $6000 \mathrm{rcf}$ for $10 \mathrm{mins}$ at $40 \mathrm{C}$ and supernatants were discarded. Bacterial pellets were resuspended in 10mL BugBuster

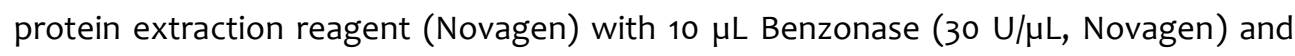


gently shaken for 20 mins at $25^{\circ} \mathrm{C}$. The proteins were then purified using His-Select $\odot$ Nickel affinity columns (Sigma Aldrich) into an elution buffer of $50 \mathrm{mM} \mathrm{NaH} 2 \mathrm{PO}_{2} 300$ $\mathrm{mM} \mathrm{NaCl} 250 \mathrm{mM}$ imidazole pH8. The purified TFP-fused knottins were then rebuffered into $\mathrm{pH} 7.4$ phosphate buffered saline (PBS, Sigma Aldrich) using $10 \mathrm{kDa}$-cutoff centrifugal filter units (Amicon Ultra). Concentrations of RFP and RFP-Y4 were determined from the absorbance value at $555 \mathrm{~nm}$ and an extinction coefficient of $100,000 \mathrm{M}^{-1} \mathrm{~cm}^{-1}$.

Monolayers on gold substrates for SPR. Synthesis of heptakis \{6-O-[12(thiododecyl)dodecanoyl)]-2,3-di-O-methyl\}- $\beta$-cyclodextrin ( $\beta-C D$ heptathioether) was done as reported previously. ${ }^{31}$ Standard $50 \mathrm{~nm}$ gold coated SPR sensors from from Ssens bv were cleaned by immersing them in piranha (1:3 mixture of concentrated $\mathrm{H}_{2} \mathrm{SO}_{4}$ and $30 \% \mathrm{H}_{2} \mathrm{O}_{2}$ ) for $15 \mathrm{~s}$. (Warning: piranha should be handled with caution; it can detonate unexpectedly). After thorough rinsing with MilliQ water, they were placed in absolute ethanol for $10 \mathrm{~min}$. The substrates were subsequently placed in a freshly prepared $0.1 \mathrm{mM}$ solution of $\beta-\mathrm{CD}$ heptathioether in $\mathrm{EtOH}$ and $\mathrm{CHCl}_{3}(1: 2 \mathrm{v} / \mathrm{v})$ for $16 \mathrm{~h}$ at $60^{\circ} \mathrm{C}$. The substrates were then rinsed with $\mathrm{CHCl}_{3}$, EtOH and MilliQ water.

Monolayers on glass substrates. The glass substrates were cleaned and activated by immersing in piranha for 30 mins followed by washing with water, ethanol and thorough drying. The surface was then functionalized with $\mathrm{N}$-[3(trimethoxysilyl)propyl]ethylenediamine (TPEDA) by overnight chemical vapor deposition using a vacuum desiccator. The substrates were then thoroughly cleaned using ethanol and toluene after which it was incubated in a $1 \mathrm{mM}$ solution of 1,4phenylene di-isothiocyanate (ITC) in dry toluene for $2 \mathrm{hr}$ at $50^{\circ} \mathrm{C}$. The substrates were then washed thoroughly with toluene, ethanol and water and finally incubated with a $1 \mathrm{mM}$ solution of per-6-amino- $\beta$-cyclodextrin (synthesized as reported previously ${ }^{32}$ ) in MilliQ water for $2 \mathrm{hr}$ at $50^{\circ} \mathrm{C}$. The substrates were finally washed thoroughly with water, dried and stored in $\mathrm{N} 2$ atmosphere until use.

$\boldsymbol{\beta}$-CD Silica Nanoparticles. $\beta$-CD silica nanoparticles were provided by Carlo Nicosia and were synthesized as described previously. 33 The nanoparticles were determined to have a mean diameter of $68 \pm 16 \mathrm{~nm}$ by DLS (data not shown). A stock solution of $0.2 \%$ $\mathrm{wt} / \mathrm{vol}$ was prepared in carbonate buffer corresponding to $10^{12}$ particles $/ \mathrm{mL}\left(8.6 \times 10^{-9}\right.$ $M)$. Assuming $\beta-C D$ surface density similar to known values on glass $\left(\sim 2.5 \times 10^{-11} \mathrm{~mol} \mathrm{~cm}\right.$ $\left.{ }^{2}\right),{ }^{32}$ the number of $\beta-C D$ molecules per particle can be calculated as $\sim 2500$, resulting in the stock solution containing a $\beta-C D$ concentration of $\sim 21 \mu \mathrm{M}$. For the experiments with RFP-Y4 and RFP, a final concentration of $0.05 \% \mathrm{wt} / \mathrm{vol}(\sim 4.3 \mu \mathrm{M})$ was used after resuspension in PBS buffer. $100 \mu \mathrm{L}$ of this nanoparticle solution was mixed with $100 \mu \mathrm{L}$ 46 
of appropriate protein solution and incubated at $20^{\circ} \mathrm{C}$ for 30 mins with $250 \mathrm{rpm}$ shaking. This was then spun down at 14,500 rpm for 5 mins in an Eppendorf minispin microcentrifuge. The supernatant was discarded and the nanoparticles were rinsed once in $500 \mu \mathrm{L}$ PBS and spun down again. The supernatant was then discarded and the nanoparticles were then finally resuspended in $200 \mu \mathrm{L}$ PBS.

Bacterial Cell Lysates. BL21DE3 cells harbouring the pRSETB TagRFP-Y4 plasmid were cultured overnight in LB Cam ${ }^{+} \mathrm{Amp}^{+}$medium without inducing TagRFP-Y4 production. The cells were then lysed using BugBuster protein extraction reagent (Novagen) with

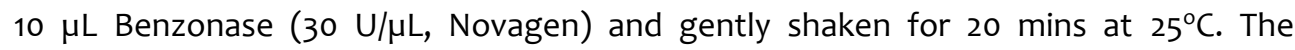
resulting lysate was then spun down at $15,000 \mathrm{~g}$ for 30 mins and the supernatant was collected. This contains all the cell lysate proteins without other cellular debris. These proteins were rebuffered into PBS using $3 \mathrm{kDa}$-cutoff centrifugal filter units (Amicon Ultra). This solution of cell lysate proteins was then mixed with appropriate concentrations of RFP and RFP-Y4 for corresponding experiments.

\subsection{References}

(1) Jonkheijm, P., Weinrich, D., Schröder, H., Niemeyer, C. M., and Waldmann, H. (2008) Chemical strategies for generating protein biochips. Angew. Chem. Int. Ed. 47, 9618-9647.

(2) Rusmini, F., Zhong, Z., and Feijen, J. (2007) Protein immobilization strategies for protein biochips. Biomacromolecules 8, 1775-1789.

(3) Sassolas, A., Blum, L. J., and Leca-Bouvier, B. D. (2012) Immobilization strategies to develop enzymatic biosensors. Biotechnol. Adv. 30, 489-511.

(4) Gu, H., Xu, K., Xu, C., and Xu, B. (2006) Biofunctional magnetic nanoparticles for protein separation and pathogen detection. Chem. Commun. 941-949.

(5) Cho, K., Wang, X., Nie, S., Chen, Z. (Georgia), and Shin, D. M. (2008) Therapeutic nanoparticles for drug delivery in cancer. Clin. Cancer Res. 14, 1310-1316.

(6) Cho, I.-H., and Irudayaraj, J. (2013) In-situ immuno-gold nanoparticle network ELISA biosensors for pathogen detection. Int. J. Food Microbiol. 164, 70-75.

(7) Xu, F., Zhen, G., Yu, F., Kuennemann, E., Textor, M., and Knoll, W. (2005) Combined affinity and catalytic biosensor: in situ enzymatic activity monitoring of surface-bound enzymes. J. Am. Chem. Soc. $127,13084-13085$.

(8) Valiokas, R., Klenkar, G., Tinazli, A., Tampé, R., Liedberg, B., and Piehler, J. (2006) Differential protein assembly on micropatterned surfaces with tailored molecular and surface multivalency. ChemBioChem 7, 1325-1329.

(9) Ma, X., and Zhao, Y. (2014) Biomedical applications of supramolecular systems based on hostguest interactions. Chem. Rev. 115(15), 7794-7839.

(10) Brinkmann, J., Cavatorta, E., Sankaran, S., Schmidt, B., van Weerd, J., and Jonkheijm, P. (2014) About supramolecular systems for dynamically probing cells. Chem. Soc. Rev. 43, 44494469 .

(11) Zhang, J., and Ma, P. X. (2013) Cyclodextrin-based supramolecular systems for drug delivery: Recent progress and future perspective. Polysacch.-Based Syst. Drug Gene Deliv. 65, 1215-1233.

(12) Loethen, S., Kim, J., and Thompson, D. H. (2007) Biomedical applications of cyclodextrin based polyrotaxanes. Polym. Rev. 47, 383-418. 
(13) Moya-Ortega, M. D., Alvarez-Lorenzo, C., Concheiro, A., and Loftsson, T. (2012) Cyclodextrinbased nanogels for pharmaceutical and biomedical applications. Int. J. Pharm. 428, 152-163.

(14) Del Valle, E. M. M. (2004) Cyclodextrins and their uses: a review. Process Biochem. 39, 10331046.

(15) Ludden, M. J. W., Ling, X. Y., Gang, T., Bula, W. P., Gardeniers, H. J. G. E., Reinhoudt, D. N., and Huskens, J. (2008) Multivalent binding of small guest molecules and proteins to molecular printboards inside microchannels. Chem. - Eur. J. 14, 136-142.

(16) Ludden, M. J. W., Péter, M., Reinhoudt, D. N., and Huskens, J. (2006) Attachment of streptavidin to $\beta$-cyclodextrin molecular printboards via orthogonal host-guest and proteinligand interactions. Small 2, 1192-1202.

(17) Ludden, M. J. W., Mulder, A., Tampé, R., Reinhoudt, D. N., and Huskens, J. (2007) Molecular printboards as a general platform for protein immobilization: a supramolecular solution to nonspecific adsorption. Angew. Chem. Int. Ed. 46, 4104-4107.

(18) Krabbenborg, S. O., Veerbeek, J., and Huskens, J. (2015) Spatially controlled out-ofequilibrium host-guest system under electrochemical control. Chem. - Eur. J. 21, 9638-9644.

(19) Voskuhl, J., Sankaran, S., and Jonkheijm, P. (2014) Optical control over bioactive ligands at supramolecular surfaces. Chem. Commun. 50, 15144-15147.

(20) González-Campo, A., Eker, B., Gardeniers, H. J. G. E., Huskens, J., and Jonkheijm, P. (2012) A supramolecular approach to enzyme immobilization in micro-channels. Small 8, 3531-3537.

(21) Yang, L., Gomez-Casado, A., Young, J. F., Nguyen, H. D., Cabanas-Danés, J., Huskens, J., Brunsveld, L., and Jonkheijm, P. (2012) Reversible and oriented immobilization of ferrocenemodified proteins. J. Am. Chem. Soc. 134, 19199-19206.

(22) Mulder, A., Onclin, S., Péter, M., Hoogenboom, J. P., Beijleveld, H., Maat, J. ter, García-Parajó, M. F., Ravoo, B. J., Huskens, J., van Hulst, N. F., and Reinhoudt, D. N. (2005) Molecular printboards on silicon oxide: lithographic patterning of cyclodextrin monolayers with multivalent, fluorescent guest molecules. Small 1, 242-253.

(23) John Xavier, S. S., Karthikeyan, C., Gnana kumar, G., Kim, A. R., and Yoo, D. J. (2014) Colorimetric detection of melamine using $\beta$-cyclodextrin-functionalized silver nanoparticles. Anal. Methods 6, 8165-8172.

(24) Xu, C., Wang, X., Wang, J., Hu, H., and Wan, L. (2010) Synthesis and photoelectrical properties of $\beta$-Cyclodextrin functionalized graphene materials with high bio-recognition capability. Chem. Phys. Lett. 498, 162-167.

(25) Horský, J., and Pitha, J. (1994) Inclusion complexes of proteins: Interaction of cyclodextrins with peptides containing aromatic amino acids studied by competitive spectrophotometry. $J$. Incl. Phenom. Mol. Recognit. Chem. 18, 291-300.

(26) Rekharsky, M. V., and Inoue, Y. (1998) Complexation thermodynamics of cyclodextrins. Chem. Rev. 98, 1875-1918.

(27) Rekharsky, M., and Inoue, Y. (2000) Chiral recognition thermodynamics of $\beta$-cyclodextrin: the thermodynamic origin of enantioselectivity and the enthalpy-entropy compensation effect. J. Am. Chem. Soc. 122, 4418-4435.

(28) Merzlyak, E. M., Goedhart, J., Shcherbo, D., Bulina, M. E., Shcheglov, A. S., Fradkov, A. F., Gaintzeva, A., Lukyanov, K. A., Lukyanov, S., Gadella, T. W. J., and Chudakov, D. M. (2007) Bright monomeric red fluorescent protein with an extended fluorescence lifetime. Nat Meth 4, 555-557.

(29) Uhlenheuer, D. A., Wasserberg, D., Haase, C., Nguyen, H. D., Schenkel, J. H., Huskens, J., Ravoo, B. J., Jonkheijm, P., and Brunsveld, L. (2012) Directed supramolecular surface assembly of snap-tag fusion proteins. Chem. - Eur. J. 18, 6788-6794.

(30) R, L., Y, D., and X, H. (2012) 2.2 Angstrom crystal structure of TagRFP-T. 
(31) Beulen, M. W. J., Bügler, J., Lammerink, B., Geurts, F. A. J., Biemond, E. M. E. F., van Leerdam, K. G. C., van Veggel, F. C. J. M., Engbersen, J. F. J., and Reinhoudt, D. N. (1998) Self-assembled monolayers of heptapodant $\beta$-cyclodextrins on gold. Langmuir 14, 6424-6429.

(32) Onclin, S., Mulder, A., Huskens, J., Ravoo, B. J., and Reinhoudt, D. N. (2004) Molecular printboards: monolayers of $\beta$-cyclodextrins on silicon oxide surfaces. Langmuir 20, 5460-5466.

(33) Mahalingam, V., Onclin, S., Péter, M., Ravoo, B. J., Huskens, J., and Reinhoudt, D. N. (2004) Directed self-assembly of functionalized silica nanoparticles on molecular printboards through multivalent supramolecular interactions. Langmuir 20, 11756-11762. 
Chapter 2 


\section{Chapter 3}

\section{Supramolecular surface immobilization of knottin derivatives for dynamic display of high affinity binders}

Knottins are known as a robust and versatile class of miniprotein scaffolds for the presentation of high-affinity binding peptides, however up to date their application in biomaterials, biological coatings and on surfaces has not been explored. We have developed a strategy to recombinantly synthesize a b-trypsin inhibitory knottin with supramolecular guest tags that enable it to adhere to self-assembled monolayers of the supramolecular host cucurbit[8]uril ( $\mathrm{CB}[8])$. We have described a strategy to easily express knottins in $\mathrm{E}$. coli by conjugating them to a fluorescent protein after which they are cleaved and purified. Various knottin constructs that varied in the number and position of the supramolecular tag at either the $\mathrm{N}$ - or C-termini or at both ends have been verified for their trypsin inhibitory function and $\mathrm{CB}[8]$-binding properties in solution and on surfaces. All the knottin constructs showed strong inhibition of trypsin with inhibition constants between 10-30 $\mathrm{nM}$. Using microscale thermophoresis, we determined that the supramolecular guest tags on the knottins bind $\mathrm{CB}[8]$ with a $K_{d}$ of $\sim 6 \mu M$ in solution. At the surface strong divalent binding has been determined with a $K_{d}$ of $0.75 \mu M$ in the case of the knottin with two supramolecular guest tags whereas only weak monovalent binding occurred when only one guest tag was present. We also showed successful supramolecular surface immobilization of the knottin using $C B[8]$ and proved that they can be used to immobilize 8-trypsin at the surface.

The work in this chapter was done along with Mark de Ruiter and is part of the publication: Sankaran, S., de Ruiter, M., Cornelissen, J. J. L. M., and Jonkheijm, P. (2015) Supramolecular surface immobilization of knottin derivatives for dynamic display of high affinity binders. Bioconjugate Chem. 26, 1972-1980. 


\subsection{Introduction}

Knottins are a very interesting class of miniproteins since their cystine-stabilized knot structure not only confers upon them very high thermal stability and resistance against proteolytic enzymes but also allows for extensive modification of the peptide sequences on their loops. ${ }^{1}$ Apart from the disulfide-bridge forming cysteines and a few other stabilizing amino acids, the residues on the loops can almost be completely changed. ${ }^{2}$ Taking advantage of these properties, nature has produced hundreds of knottins with very high activities as protease inhibitors, neurotoxins in spider and scorpion venom, insecticides from plants and antimicrobial agents as can be seen in the open access online knottin database. ${ }^{3,4}$ Several of these naturally occurring knottins have also been tested as possible analgesics, anti-malarials and pesticides. ${ }^{5}$

The potential of these simple proteins as stable molecular scaffolds has promoted their extensive usage in phage and bacterial display systems to identify high affinity binding peptides for various target proteins related to growth factors, cancer, HIV and in-vivo imaging. ${ }^{6}$ These display techniques, combined with the fact that their small size enables them to be chemically synthesized, have hailed these molecules as potential substitutes for antibodies. ${ }^{7,8}$ Daugherty and co-workers have successfully developed a simple bacterial display system by which they were able to determine high affinity binding knottins towards thrombin and neuropilin. ${ }^{9,10}$ Chiche et al. have developed a truncated form of the knottin EETI-II, called Min-23, with which they were able to identify high affinity binders for various target proteins. ${ }^{11,12}$ Similarly, phage display systems have been used to identify modified Min-23 miniproteins that were able to bind growth factors such as VEGF. ${ }^{13,14}$ Cochran and coworkers through rational design strategies, developed knottins containing RGD with flanking residues that were able to bind different integrins with different levels of affinity. ${ }^{15,16}$ Further, to expand upon the functionality of the exposed groups, strategies have been developed to chemically conjugate various synthetic molecules and non-natural amino acids. ${ }^{17,18}$ Such constructs have been successfully shown as effective imaging agents using conjugated fluorescent dyes and radiofluorinated compounds. ${ }^{19,20}$

Due to such possibilities of effectively displaying a wide variety of peptide sequences made of either natural or non-natural amino acids, these miniprotein scaffolds are excellent candidates for fabricating highly stable bioactive surfaces and materials for research, biosensors and even implants. Their loop-structure would also ensure better solvent-exposure of the incorporated peptide sequences and their size ( $3-5 \mathrm{kDa})$ allows for tighter, oriented packing than antibodies and nanobodies. ${ }^{21}$ Since they 52 
contain only about 30 amino acids that fold into a tight cage-like structure, non-specific interactions with surfaces and proteins are also substantially reduced compared to larger constructs. However, so far, there are no studies attempting to fabricate biofunctional surfaces using knottins.

Several strategies have been explored to incorporate various other functional molecules at surfaces, for example, using self-assembled monolayers (SAMs), polymers, nanoparticles, lipid bilayers etc. ${ }^{22,23}$ Furthermore, supramolecular chemistry has enabled the introduction of dynamics and responsiveness in these functional surfaces. ${ }^{24,25}$ These platforms are also developing as powerful tools for studying complex biological processes such as cellular adhesion, migration, cell-cell interactions etc. $^{26}$ In particular, synthetic host-guest systems have recently been vigorously explored for these purposes due to their similarity with protein-protein interactions found in nature. ${ }^{27}$ By careful design of the host-guest components, spatial and temporal control of the interactions can be introduced. ${ }^{28,29}$ Stupp and coworkers have been able to create a matrix, using alginate and $\beta$-cyclodextrin, with controllable celladhesion properties by competitive exchange of functionalized guest molecules. ${ }^{30} \mathrm{We}$ had previously developed a supramolecular platform for the photo-responsive display of bioactive ligands using azobenzene glycoconjugates and $\beta$-cyclodextrin SAMs. ${ }^{31}$ We were also able to develop a supramolecularly addressable bacterial strain and incorporate it as a living component with SAMs bearing cucurbit[8]uril (CB[8]) host molecules. $^{32}$ In order to accomplish this, we genetically modified one loop of a Min-23 miniprotein to carry a $\mathrm{CB}[8]$-binding motif. This was then linked to a transmembrane protein so that it would get displayed on the bacterial surface. The bacterial display system clearly showed specific binding towards $\mathrm{CB}$ [8], causing aggregation of bacteria in solution through multivalent interactions and allowing the adhesion of bacterial cells onto $\mathrm{CB}$ [8] SAMs while still retaining their motility. This study highlighted the potential of developing knottins with supramolecular functionalities.

In the last decade there has been a keen interest in exploring the possibility of applying the $C B[n]$ family of host molecules for biomedical applications. $C B[n] s$ are hollow pumpkin shaped macrocyclic molecules made of glycouril monomer units. ${ }^{33}$ They have a hydrophobic cavity and polar rims lined with ureido-carbonyl oxygens allowing them to bind a variety of aromatic cations with micromolar affinities. ${ }^{34,35}$ Among the $C B[n]$ family members, $C B[8]$ happens to be especially interesting since the cavity is large enough to simultaneously encapsulate two aromatic guest molecules. ${ }^{36}$ This enables the possibility to construct e.g. dual responsive nanoparticle systems when two different guest molecules that are affected by different external stimuli are included in 
the cavity. ${ }^{37}$ We and others have developed strategies to assemble $\mathrm{CB}$ [8] on biologically relevant surfaces. ${ }^{29,38,39}$ Furthermore, we have also demonstrated the ability to dynamically display bioactive ligands for cell adhesion and triggered release using electrochemical stimuli. ${ }^{29,40}$ These studies indicate the potential of $\mathrm{CB}$ [8] for developing highly dynamic and responsive smart surfaces for biomedical applications and research.

Here, we have explored the possibility of constructing knottins that can be specifically immobilized on $\mathrm{CB}[8]$ monolayers and still bind their target protein (Figure 3.1a). A strategy was developed to recombinantly synthesize a $\beta$-trypsin inhibitor knottin with supramolecular guest tags that enable it to adhere to SAMs of $C B[8]$. The peptide sequence "GGWGG" has been shown to bind $C B[8]$ with low micromolar affinities ${ }^{41}$ and we designed four different knottin constructs (K) bearing this motif at either the $\mathrm{N}$ terminus $\left(\mathrm{K}_{1} \mathrm{~N}\right)$, C-terminus $\left(\mathrm{K}_{1} \mathrm{C}\right)$, both termini $\left(\mathrm{K}_{2}\right)$ or none $\left(\mathrm{Ko}_{0}\right)$ (Figure $\left.3.1 \mathrm{~b}\right)$. In the cases of $\mathrm{K}_{1} \mathrm{~N}, \mathrm{~K}_{1} \mathrm{C}$ and $\mathrm{Ko}$, the termini without the GGWGG motif have GGGGG motifs instead. First, we describe a strategy to easily express these knottins in $E$. coli by conjugating them to a fluorescent protein after which it is cleaved and purified. These knottins were proven to be functional using a trypsin inhibitor assay and their $\mathrm{CB}[8]$ binding characteristics in solution were compared. We also tested supramolecular surface immobilization of these knottin using $\mathrm{CB}[8]$ and proved that even at the surface, these miniproteins show functionality. For the first time, the binding of a functional bivalent guest molecule $\left(\mathrm{K}_{2}\right)$ has been studied in comparison to monovalent guests $\left(\mathrm{K}_{1} \mathrm{C} / \mathrm{N}\right)$ on such ternary complex based SAMs. The bivalent guest had a higher binding affinity and was the only one with which trypsin was clearly immobilized on the supramolecular surface. 

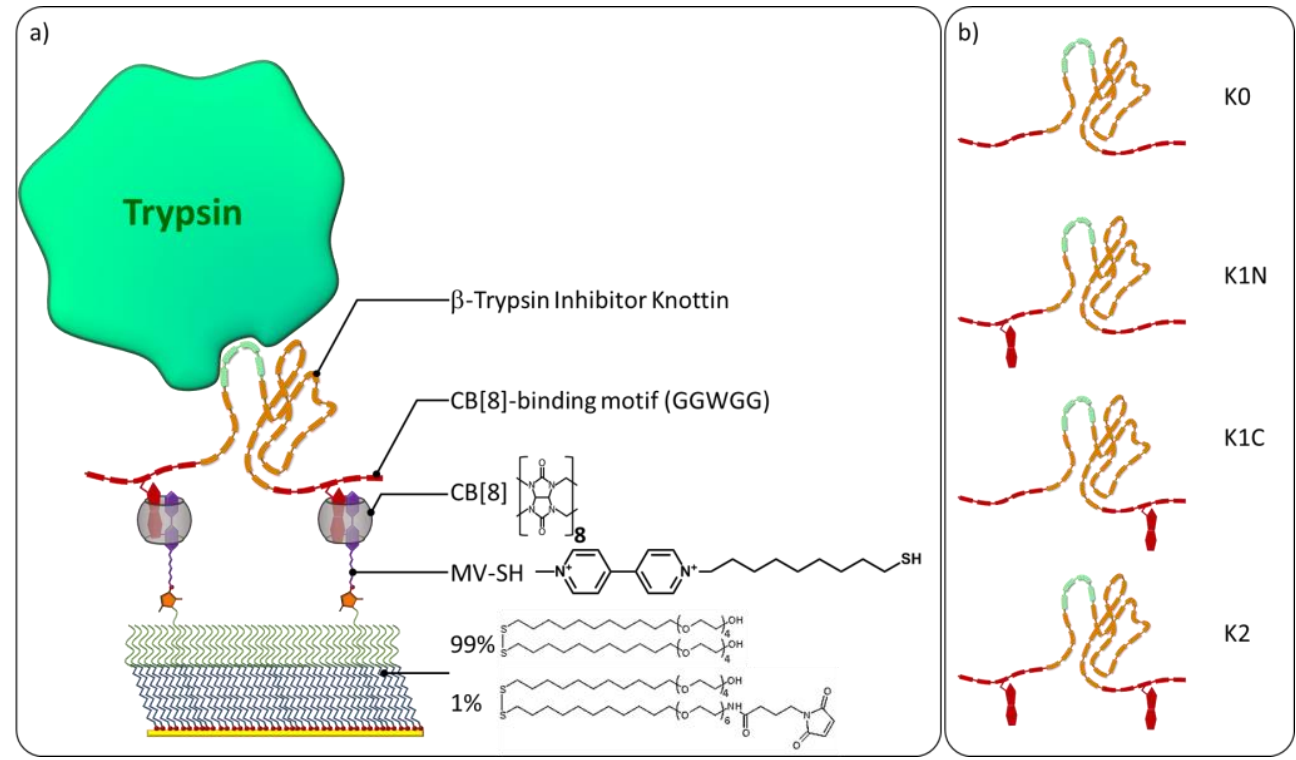

Figure 3.1. a) Schematic depicting the supramolecular surface immobilization of a genetically modified functional b-trypsin inhibitor knottin onto a $\mathrm{CB}$ [8]-presenting SAM on gold. b) Depictions of the four different $\mathrm{CB}[8]$-binding knottin constructs used in this study. The presence of tryptophan $(W)$ in the $\mathrm{CB}[8]$-binding motif (red) has been represented by its indole side chain.

\subsection{Results and Discussions}

\subsubsection{Design and recombinant synthesis of knottins}

As shown in Figure 3.2a, we developed a modified strategy to express and purify our knottin constructs. Due to their multiple cysteines and poor solubility during expression in E. coli, knottins are usually formed in inclusion bodies from which they must be solubilized, denatured and refolded. Kolmar and co-workers developed a strategy to circumvent this problem by fusing the knottin to barnase, which improved solubility and caused it to be secreted into the periplasm where disulfides form more easily in an oxidative environment. ${ }^{42}$ Based on these concepts, we devised a modified strategy to further improve the solubility and folding of our knottin constructs. Solubility was improved by genetically fusing the knottins to the $\mathrm{C}$ terminal of a $\mathrm{His}_{6}$-tagged teal fluorescent protein (TFP) with a linker containing an enterokinase cleavage site (DDDDK). Furthermore, TFP was chosen since it allowed us to visualize the protein and perform fluorescence based analysis. Disulfide bond formation was improved (as 
shown by Ellman's reagent) by expressing these constructs in Rosetta Gami 2 E. coli cells, a specially engineered commercially available strain in which certain thioreductases are mutated. These fusion proteins were purified using Ni-NTA affinity columns and characterized using SDS-PAGE (Figure 3.7, see in Experimental section) and size-exclusion FPLC (Figure 3.2b (K2) and Figure S3.2a (Ko, K1N, K1C, K2, in Section 3.7, Supporting information). We were able to obtain an average of $34 \mathrm{mg}$ of these fusion proteins ( $\sim 4.7 \mathrm{mg}$ knottin) from $1 \mathrm{~L}$ of bacterial culture. Next, enterokinase was used to cleave the knottin from the TFP (Figure 3.2c). As expected, the absorbance at $\lambda$ $=280 \mathrm{~nm}$ varied for each construct, depending on the number of tryptophan residues present, $\mathrm{K} 2$ showing the highest and Ko showing the least (Figure 3.8a, see in Experimental section). Surprisingly, each knottin construct eluted at different volumes during the size exclusion chromatography even though their sizes are not considerably different (Figure 3.8a, see in Experimental section). K2 eluted at $16 \mathrm{~mL}, \mathrm{~K}_{1} \mathrm{~N}$ and $\mathrm{K}_{1} \mathrm{C}$ at $15 \mathrm{~mL}$ and $\mathrm{Ko}$ at $14 \mathrm{~mL}$ indicating that the secondary structure of $\mathrm{K} 2$ was the smallest followed by $\mathrm{K}_{1} \mathrm{~N}$ and $\mathrm{K}_{1} \mathrm{C}$ and $\mathrm{Ko}$ was the bulkiest. This was a counter-intuitive outcome since the molecular weights of these constructs, verified by MALDI-TOF analysis (Figure $3.8 \mathrm{~b}$, see in Experimental section), followed the trend of $\mathrm{K}_{2}>\mathrm{K}_{1} \mathrm{~N}=\mathrm{K}_{1} \mathrm{C}>\mathrm{Ko}_{0}$ due to the presence of the tryptophan residues. The most likely explanation for this behavior is that the indole side chains of tryptophan, being hydrophobic, fold into the hydrophobic core of the knottin causing these constructs to be more compact. Due to the considerable difference in size between the knottins $(\sim 4.8 \mathrm{kDa})$ and the $\mathrm{His}_{6}$-tagged TFP ( $27 \mathrm{kDa}$ ) (Figure 3.2C), they were separated using a centrifugal filter unit with a 10 kDa cut-off. Smaller impurities were removed using a centrifugal filter unit with a $3 \mathrm{kDa}$ cut-off. Knottins purified in this manner were analyzed by FPLC (Figure 3.2d) and MALDI-TOF (Figure 3.8b, see in Experimental section) and indicated a lack of free thiols (Ellman's reagent). The enterokinase cleavage and purification steps resulted in nearly a $70 \%$ yield of pure knottin providing us with an average of $3.2 \mathrm{mg}$ purified knottins per $1 \mathrm{~L}$ of bacterial culture, which is comparable to yields reported by Kolmar and coworkers, ${ }^{42}$ yet, production and cleavage was easier by the presence of TFP while the TFP-fused knottins can be used for subsequent detailed fluorescent based binding studies. 

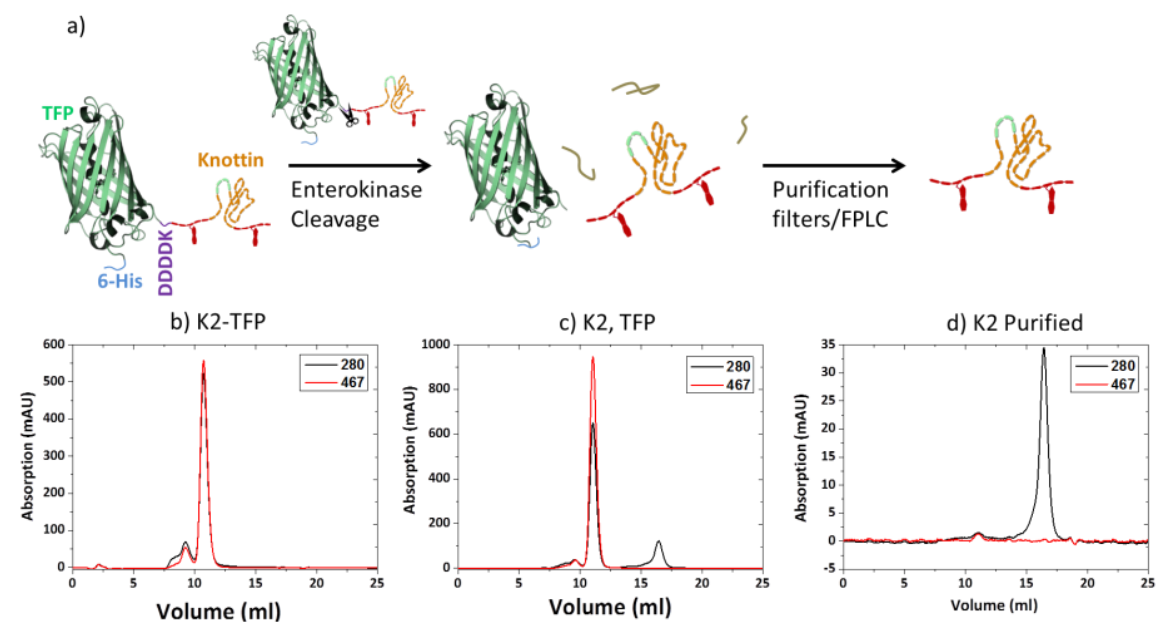

Figure 3.2. (a) Schematic representation of the steps involved in synthesizing and purifying the knottin constructs. Size exclusion FPLC traces of $b$ ) the recombinantly synthesized fusion protein (K2-TFP), c) the protein mixture (K2 and TFP) after enterokinase cleavage and d) K2 knottin purified using centrifugal filter units.

\subsubsection{Evaluating trypsin inhibitory functionality of knottin constructs}

To ensure that our purified knottin constructs were still functional, we performed a trypsin inhibitor assay (Figure 3.3). For this purpose, we followed the cleavage of $\mathrm{Na}$ benzoyl-L-arginine ethyl ester (BAEE) by measuring the emerging absorbance at $\lambda=260$ $\mathrm{nm}$ over time. This molecule gets cleaved by $\beta$-trypsin as shown in Figure 3.3a and the product has a red-shifted absorbance peak at $253 \mathrm{~nm}$ wavelength. It was clearly seen that the rate of cleavage reduced in the presence of the knottins. Since our knottins are known to inhibit $\beta$-trypsin by blocking its active site, ${ }^{43}$ competitive inhibition kinetics would be expected where inhibited reactions have the same $V_{\max }$ value as the uninhibited reactions but a lower $K_{m}$ value. However, fitting all plots with the MichaelisMenten equation (eq 3.1) revealed that the presence of knottins caused a considerable drop in both the $V_{\text {max }}$ and $K_{m}$ values (Table 3.1, see in Experimental section).

$$
v=\frac{V_{\max }[S]}{K_{m}+[S]}
$$

where $v$ is the reaction rate $\left(\mathrm{s}^{-1}\right), V_{\max }$ is the maximum reaction rate $\left(\mathrm{s}^{-1}\right),[S]$ is the BAEE concentration $(M)$ and $K_{m}$ is the Miachelis constant $(M)$, the concentration of BAEE where the reaction rate is half of $V_{\max }$. 
This effect could correspond to non-competitive inhibition or irreversible inhibition. Non-competitive inhibition is highly unlikely since the knottins are known to bind and block the active site of $\beta$-trypsin. Also, since the inhibition occurs at much lower inhibitor concentrations than that of BAEE, high binding strength is expected, suggesting irreversible inhibition. However, in case of irreversible inhibition kinetics, it is known that $V_{\max }$ of the inhibited reaction drops compared to the uninhibited reaction but $K_{m}$ stays the same. This indicated that the high binding affinity of these knottins must have resulted in pseudo-irreversible inhibition kinetics. Consequently, apparent inhibitor constants were determined with the following formula (eq 3.2), which describes the case of competitive tight binding inhibitors: ${ }^{42,44}$

$$
\frac{V_{i}}{V_{0}}=1-\frac{\left[E_{T}\right]+\left[I_{T}\right]+K i_{a p p}-\left\{\left(\left[E_{T}\right]+\left[I_{T}\right]+K_{i a p p}\right)^{2}-4\left[E_{T}\right]\left[I_{T}\right]\right\}^{1 / 2}}{2\left[E_{T}\right]}
$$

where $V_{i}$ and $V_{0}$ are the $V_{\max }$ values of the inhibited and uninhibited reactions respectively, $\left[E_{T}\right]$ is the total concentration of $\beta$-trypsin, $\left[I_{T}\right]$ is the total knottin concentration and $K_{\text {iapp }}$ is the apparent dissociation constant of the enzyme-inhibitor complex. This analysis provided $K_{\text {iapp }}$ values in the low nanomolar range, similar to values obtained by Kolmar and co-workers for recombinantly produced $\beta$-trypsininhibitor knottins. ${ }^{42}$ Interestingly, the cleaved knottins showed a modest improvement in $K_{\text {iapp }}$ values $(\sim 10 \mathrm{nM})$ compared to the TFP-fused knottins $(\sim 26 \mathrm{nM})$ presumably due to reasons of steric hindrance. These results indicated that all four knottin constructs exhibited significant trypsin inhibitory functionality. 

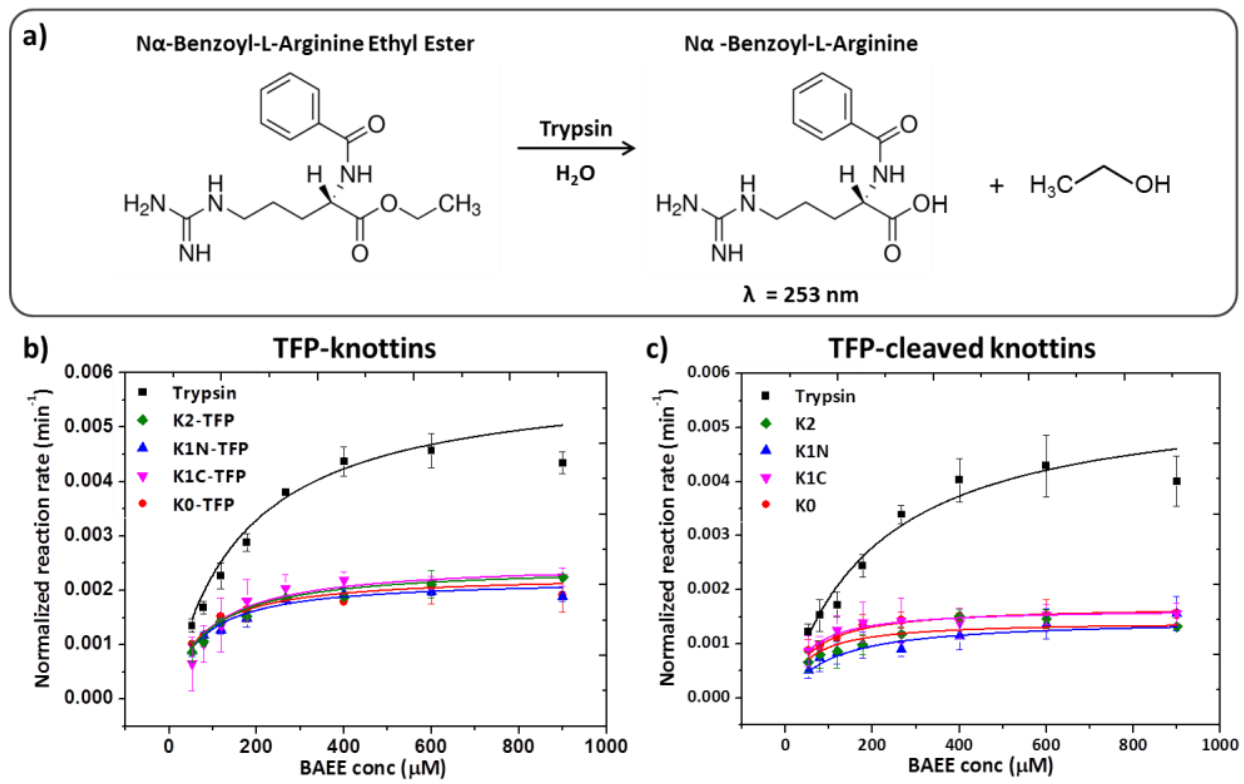

Figure 3.3. a) B-trypsin enzymatic activity assay reaction mechanism. Trypsin inhibition assay following the cleavage of different concentrations of BAEE by 6 -trypsin in the absence and presence of the different knottin constructs in (b) TFP-fused and (c) TFP-cleaved knottins. Data presented as mean $\pm S T D, n=3$. Solid lines represent fitting of the individual curves using the Michaelis-Menten (eq 3.1). $K_{\text {iapp }}$ values obtained using eq 3.2 of K2-TFP $=29 \pm 5 \mathrm{nM}, K_{1} \mathrm{~N}-\mathrm{TFP}=22 \pm 3 \mathrm{nM}, \mathrm{K}_{1} \mathrm{C}-\mathrm{TFP}=31 \pm 8 \mathrm{nM}$, Ko-TFP $=24 \pm 6 \mathrm{nM}, K_{2}=8 \pm 4 \mathrm{nM}, \mathrm{K}_{1} \mathrm{~N}=9 \pm 2 \mathrm{nM}, \mathrm{K}_{1} \mathrm{C}=12 \pm 5 \mathrm{nM} . \mathrm{Ko}_{0}=12 \pm 4 \mathrm{nM}$.

\subsubsection{Evaluating $\mathrm{CB}[8]$-binding capability of knottin constructs}

The $\mathrm{CB}[8]$ binding capability of the different knottin constructs in solution was tested using microscale thermophoresis (MST). In this technique, a solution containing a fluorescent species is locally heated and the diffusion rate of the molecules in that spot is determined by measuring the fluorescence intensity. The diffusion rate depends on the hydration shell around the fluorescent molecule and when a binding event occurs, this shell gets modified, resulting in a different rate of diffusion. For this purpose, we used the TFP-fused knottin constructs and measured the binding affinity by titrating with a range of $\mathrm{CB}[8]$ concentrations $(2 \mathrm{nM}-37.5 \mu \mathrm{M})$ in the presence of $150 \mu \mathrm{M}$ methylviologen $\left(\mathrm{MV}^{2+}\right)$ (Figure 3.4a). $\mathrm{MV}^{2+}$ forms a 1:1 binary complex with $\mathrm{CB}[8]$ allowing us to study the affinity of the knottins with this complex to form a ternary complex. Under these conditions, K1N-TFP, K1C-TFP and K2-TFP clearly exhibited changes in thermophoretic behavior with increasing $C B[8]$ concentrations. Ko-TFP did 
not show such changes in diffusion rate indicating that the $\mathrm{CB}[8]-\mathrm{MV}^{2+}$ complex binds specifically to the GGWGG motif on the other constructs at these concentrations. The low micromolar binding affinities $(\sim 6 \mu \mathrm{M})$ determined are in the expected range for such supramolecular host-guest interactions. ${ }^{41}$ No significant differences were observed between the binding affinities of the monovalent-binding constructs (K1NTFP, K1C-TFP) and the bivalent construct (K2-TFP), since the $\mathrm{CB}[8]-\mathrm{MV}^{2+}$ complex still interacts with the GGWGG motifs in a monovalent manner. However, the thermophoretic amplitudes of K2-TFP was almost equal to sum of the values of K1N-TFP and $\mathrm{K}_{1} \mathrm{C}$-TFP at each point (Figure 3.4b). This indicates the possibility of simultaneous binding $\mathrm{CB}[8]-\mathrm{MV}^{2+}$ complexes with both the GGWGG motifs on K2-TFP. Differences in thermophoretic amplitudes between $\mathrm{K}_{1} \mathrm{~N}-\mathrm{TFP}$ and $\mathrm{K}_{1} \mathrm{C}-\mathrm{TFP}$ arise due to the fact that the $\mathrm{CB}[8]-\mathrm{MV}^{2+}$ complex binds these proteins at different locations.
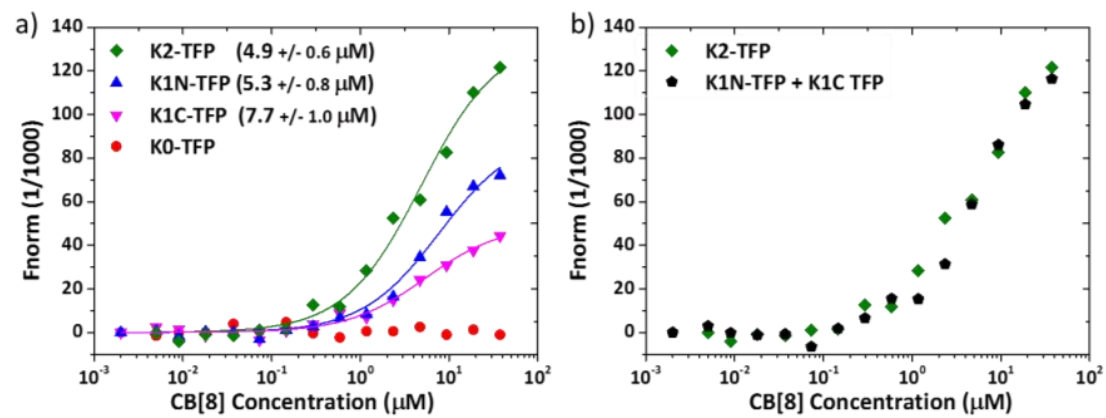

Figure 3.4. a) Thermophoretic binding curves corresponding to the interaction between the different TFP-Fused knottins and the $\mathrm{CB}[8]-\mathrm{MV}^{2+}$ complex. Solid lines represent fitting the data with the Langmuir equation and the bracketed values in the legend are the obtained dissociation constants. b) Thermophoretic binding curves of K2-TFP and the sum of K1N-TFP and K1C-TFP.

\subsubsection{Supramolecular surface immobilization of knottins}

Confident that the knottin constructs showed inhibitory effect on trypsin and were able to bind $\mathrm{CB}[8]$, we proceeded to study the valency of their interaction with $\mathrm{CB}[8]$ SAMs using surface plasmon resonance (SPR). For this purpose, we used monolayers that display $\mathrm{MV}^{2+}$ on a background of non-fouling tetraethylene glycols, as depicted in Figure 3.1a. ${ }^{40} \mathrm{CB}[8]$ was first non-covalently immobilized onto the $\mathrm{MV}^{2+}$ units and then the knottins were allowed to interact with this complex. From Figure 3.5a, it can be seen that the knottins with only one GGWGG motif ( $\mathrm{K}_{1} \mathrm{~N}$ and $\mathrm{K}_{1} \mathrm{C}$ ) show weaker binding compared to $\mathrm{K} 2$ at the concentrations used $(0.15-3 \mu \mathrm{M})$. At higher concentrations up to $30 \mu \mathrm{M}$, even with Tween-20 added, the knottin solutions become turbid over time 
signified by broadening in the UV/vis absorption spectrum (Figure S3.1, Section 3.7, Supporting Information). This indicated aggregation of knottins into clusters possibly due to their relatively poor water solubility. Tween-20 was added as a non-ionic amphiphilic detergent used for solubilizing membrane proteins. It can prevent protein clustering by masking hydrophobic domains. It has also been extensively used to prevent non-specific binding in protein-protein interactions, for example, in immunoassays. However, irrespective of the position of the supramolecular guest tag, either at the $\mathrm{N}$ or $\mathrm{C}$ terminus, the SPR binding profile was nearly identical. Ko did not exhibit surface adhesion confirming that immobilization of the other constructs occurred only through specific interactions between $\mathrm{CB}[8]$ and the GGWGG motif in agreement with the MST results in solution. When maximum SPR responses of K2 were plotted against corresponding concentrations and fitted with the Langmuir adsorption equation, assuming both GGWGG motifs can simultaneously bind to the $\mathrm{CB}[8]$ SAM, a dissociation constant $\left(K_{d}\right)$ of $0.75 \pm 0.06 \mu \mathrm{M}$ was obtained (Figure $3.5 \mathrm{~b}$ ). This value is lower than the binding constant of a single GGWGG motif to the CB8-MV ${ }^{2+}$ complex. This improvement in binding affinity due to divalent interactions is comparable to what has been reported with similar supramolecular host-guest systems in buffered conditions with Tween-20.45 Binding plots of $\mathrm{K} 1 \mathrm{~N}$ and $\mathrm{K}_{1} \mathrm{C}$ indicated that their binding was still in the linear regime at these concentrations $(0.15-3 \mu \mathrm{M})$, confirming less stable binding between the monovalent constructs and $\mathrm{CB}[8]$ SAMs. This difference in binding strength was also observed when rinsing the surfaces with buffer. The divalent $\mathrm{K} 2$ construct was only partly removed while the monovalent constructs $\left(\mathrm{K} 1 \mathrm{~N}, \mathrm{~K}_{1} \mathrm{C}\right)$ were completely removed in the same period of time. The signal of the divalent K2-TFP drops to nearly half of its saturation value in ca. 14 minutes. Surface adhesion of TFP-fused knottin constructs were also followed using SPR (Figure S3.2, Section 3.7, Supporting Information). Even though significant non-specific adhesion was seen with Ko-TFP, which could not be prevented by adding Tween-20, better surface adhesion was clearly seen with the bivalent K2-TFP compared to the monovalent K1N-TFP and K1C-TFP. Nonspecific adhesion most likely occurs due to multiple weak interactions between $\mathrm{CB}$ [8] and the solvent-exposed aromatic amino acids present in the fluorescent proteins as observed before by others. ${ }^{46}$ These results collectively indicate that specific supramolecular host-guest binding occur between the surface and the knottin constructs after cleaving off the TFP parts and confirm that a divalent interaction occurs in the assembly of the $\mathrm{K} 2$ knottin constructs onto $\mathrm{CB}[8]$ SAMs. 

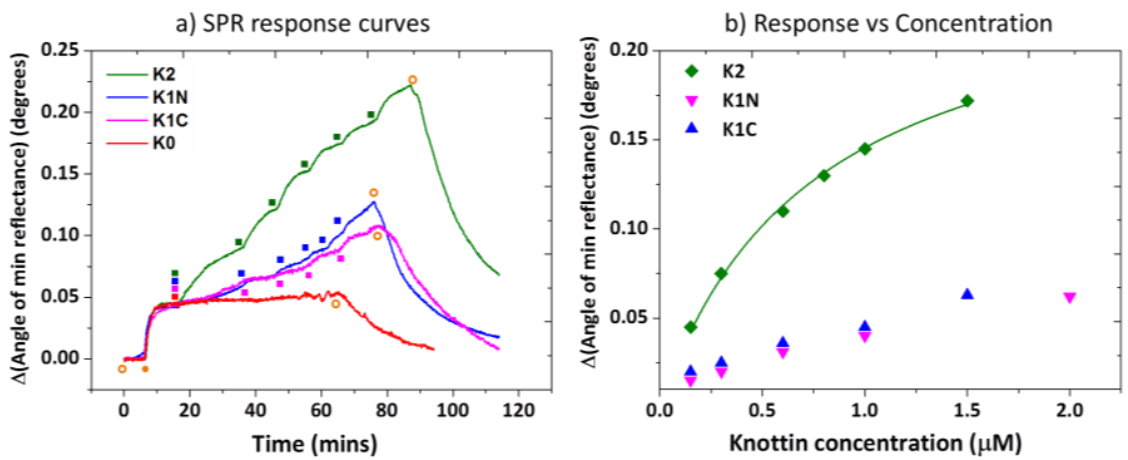

Figure 3.5. a) SPR response curves corresponding to the binding of each knottin construct on $C B[8]$ SAMs. Hollow circles (o) represent buffer, filled circle ( $\odot$ )represents $50 \mu M C B[8]$, solid squares ( $\square$, $\square, \square, \square$ ) represent increasing concentrations of corresponding knottin constructs (Ko - $1 \mu M, K 1 \mathrm{~N}$ $\left.0.15,0.3,0.6,1,2,3 \mu \mathrm{M}, \mathrm{K}_{1} \mathrm{C}-0.15,0.3,0.6,1,1.5 \mu \mathrm{M}, \mathrm{K} 2-0.15,0.3,0.6,0.8,1,1.5 \mu \mathrm{M}\right)$. Titration of various knottin constructs were always performed in the presence of $50 \mu \mathrm{M} \mathrm{CB}[8]$ in solution. b) Saturating SPR response for each concentration of $K 2$ and corresponding maximum SPR response of $\mathrm{K}_{1} \mathrm{~N}$ and $\mathrm{K}_{1} \mathrm{C}$ for the same time interval at each concentration. Solid line represents fitting of $\mathrm{K}_{2}$ values with the Langmuir adsorption equation $\left(K_{d}=0.75 \pm 0.06 \mu \mathrm{M}\right)$.

To test the applicability of the $\mathrm{CB}[8]$-binding knottins in fabricating dynamic protein arrays, we attempted patterning them by supramolecular microcontact printing. ${ }^{47}$ First, we tested whether the knottin constructs would bind specifically to non-covalent arrays of $\mathrm{CB}$ [8]. For this purpose, circle-patterns of $\mathrm{CB}$ [8] were first printed on surfaces displaying $\mathrm{MV}^{2+}$. To visualize knottin adhesion, we incubated these surfaces with the TFP-fused knottins. Fluorescent arrays were clearly seen in the cases of K1N-TFP, K1CTFP and K2-TFP but not with Ko-TFP as shown in Figure 3.6a. As a second check to verify specific surface adhesion following ternary complex formation, we used $\mathrm{CB}$ [7] as a negative control. Since $\mathrm{CB}[7]$ has a smaller cavity, it can accommodate only one guest at a time. ${ }^{34}$ Consequently, $\mathrm{CB}[7]$ should be able to bind to $\mathrm{MV}^{2+}$ at the surface but not form a ternary complex with the knottins. As expected, we did not observe any fluorescent arrays after incubating all four TFP-fused knottins with $C B$ [7]-patterned surfaces.

As the knottin constructs adhere to $C B[8]$-arrays, dissociation of $C B[8]$ is expected to occur in parallel, resulting in the weakly fluorescent arrays seen in Figure 3.6a. To increase the density of the bound knottins and simplify the process of making such arrays, we tested a different strategy. Mixtures of $\mathrm{CB}[8]$ and the TFP-fused knottins 62 
were printed on surfaces displaying $\mathrm{MV}^{2+}$ followed by a 20 min wash in buffer with mild shaking. In Figure 3.6b it can be seen that after printing, fluorescent arrays were visible in the cases of both K2-TFP and Ko-TFP. After the wash step, $60 \%$ of the original fluorescence of the K2-TFP arrays remained, whereas the fluorescence of the Ko-TFP arrays almost entirely disappeared ( $<10 \%$ remaining). The final intensity of K2-TFP arrays using this strategy was significantly higher $(\sim 7 \mathrm{x})$. Using this strategy, we tested the possibility of binding trypsin to arrays of TFP-cleaved $\mathrm{CB}[8]$-binding knottins. To visualize trypsin on the arrays, we conjugated a Cy3-NHS ester reactive dye to the amino groups of the protein (Tryp-Сy3). Arrays of all four TFP-cleaved knottin constructs were incubated with Tryp-Cy3 for $10 \mathrm{~min}$. Clear Cy3-fluorescent arrays were observed only on surfaces with $\mathrm{K} 2$ (Figure 3.6c). Due to less stable binding capability of the monovalent $\mathrm{CB}[8]$-binding TFP-cleaved knottins (K1N, K1C), as seen in the SPR measurements, the surface density of $K 1$ constructs is lower after printing and washing and complete dissociation might have occurred after the complete process. In the case of $\mathrm{K}_{2}$, bivalent interactions result in a higher affinity allowing for a greater surface density of $K_{2}$ constructs after printing and washing and slower dissociation during the subsequent steps yields suitable arrays of trypsin binding knottins. These results further highlight that significant differences exist in binding capabilities between monovalent and bivalent guests on ternary-complex forming surfaces. Based on these array experiments, we verified the interaction of $\beta$-trypsin on a $M^{2+}-\mathrm{CB}[8] \mathrm{SAM}$ displaying K2 using SPR (Figure S3.3, Section 3.7, Supporting Information). As expected, $\beta$-trypsin interacted with this SAM and rinsing with buffer caused dissociation, confirming that reversible surface adhesion of $\beta$-trypsin was achieved through supramolecular interactions. 
a) TFP-fused Knottin patterns
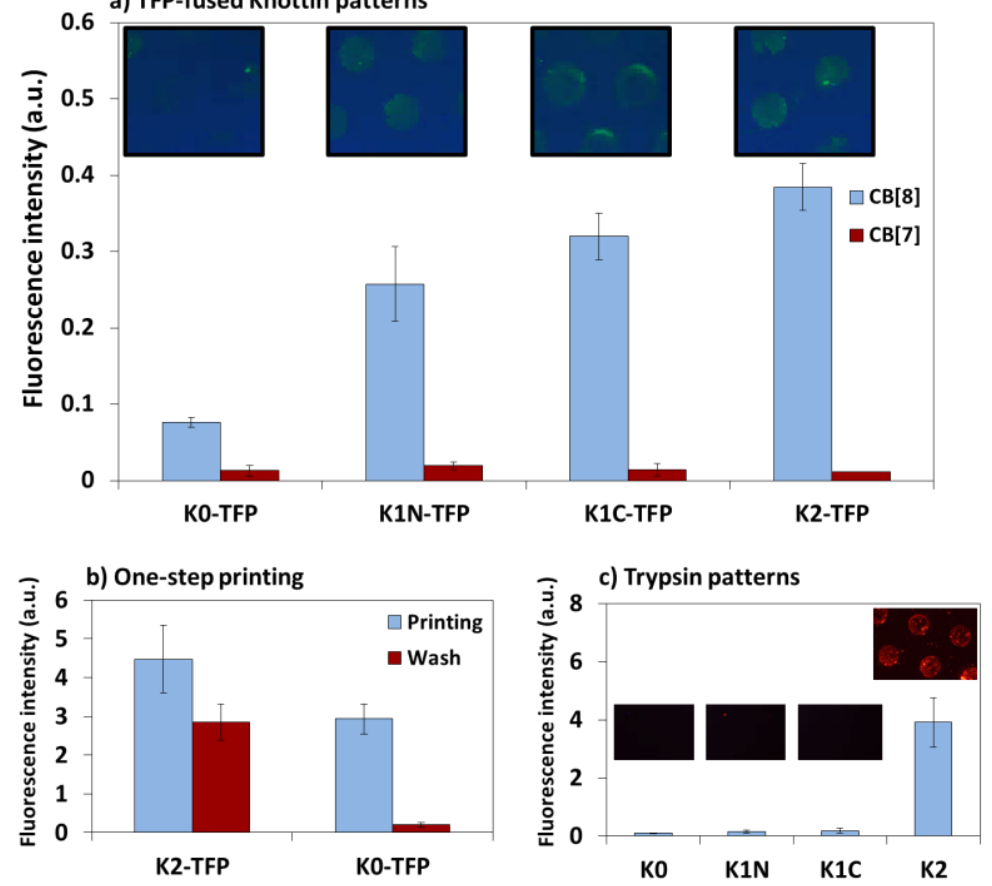

Figure 3.6. a) Fluorescence intensity of the different TFP-fused knottins immobilized on surfaces patterned with $\mathrm{CB}[8]$ (blue columns) and $\mathrm{CB}[7]$ (red columns). b) Fluorescence intensities of patterns formed by printing a mixture of $\mathrm{CB}[8]$ and the TFP-fused knottins (blue columns) and after washing the patterns in buffer for 20 mins (red columns). c) Fluorescence intensities of Tryp- $\mathrm{Cy}_{3}$ immobilized on patterned surfaces of the four different cleaved knottins. Fluorescence intensities of the patterned spots were determined and subtracted from the background in all cases. Data presented as mean \pm STD, $n=4$.

\subsection{Conclusions}

In summary, $\mathrm{CB}[8]$-binding knottins have been constructed and used to fabricate functional knottin-based supramolecular surfaces that are able to bind their protein binding target. A simple strategy has been developed to recombinantly express and purify knottins by genetically fusing them to TFP using an enterokinase cleavable linker. The TFP-fused knottins have been directly used for fluorescence based analysis and to visualize them on surfaces. Both TFP-fused and TFP-cleaved knottin constructs have been tested for their ability to inhibit $\beta$-trypsin and to bind $C B$ [8]. All knottin constructs inhibited $\beta$-trypsin with low $\mathrm{nM}$ affinities and those containing $\mathrm{CB}[8]$-binding motifs 
bound to $\mathrm{CB}[8]$ with low $\mu \mathrm{M}$ affinities. The knottin constructs have also been immobilized to surfaces displaying $\mathrm{MV}^{2+}$ by $\mathrm{CB}[8]$-mediated ternary complex formation. Differences in surface adhesion capabilities were observed between monovalent and bivalent $\mathrm{CB}[8]$-binding knottin constructs. Improved specific binding could be observed when TFP was cleaved off the knottins presumably because of steric hindrance and non-specific interactions of the TFP with $\mathrm{CB}[8]$. Finally, surface adhesion of trypsin occurred only on surfaces patterned with the divalent knottin construct, K2, due to stronger surface adhesion characteristics.

This study demonstrates, for the first time, the possibility of developing functional knottins that can be incorporated with a supramolecular host-guest system and capture a target protein. Knottins are a robust and versatile class of miniprotein scaffolds for the presentation of high-affinity binding peptides. Knottins that bind biomolecules such as growth factors, hormones and cell-surface receptors, could prove to be powerful components that can be integrated with biologically relevant supramolecular materials like surface coatings, hydrogels, soft nanoparticles, polymers etc.

\subsection{Acknowledgements}

We would like to thank Emanuela Cavatorta for providing $\mathrm{MV}^{2+}$-SH, Jenny Brinkmann for providing $20 \mathrm{~nm}$ gold substrates and Regine van der Hee for her assistance in performing the MALDI-TOF measurements. The facilities of the BioNanoLab of the $\mathrm{MESA}^{+}$Institute for Nanotechnology are highly appreciated.

\subsection{Experimental Section}

Materials. Cucurbit[8]uril, cucurbit[7]uril, $\beta$-trypsin from bovine pancreas and methylviologen were purchased from Sigma-Aldrich. The disulphides (Bis-1-(11\{tetraethylene glycol\}-undecyl) disulfide and $\mathrm{N}$-\{2-(2,5-dioxo-2,5-dihydro-pyrrol-1-yl)ethyl\}-[2-[11-(11-(tetraethylene glycol)-undecyldisulfanyl)-undecyloxy]-hexaethylene glycol-acetamide]) for SAM preparation were purchased from ProChimia. Alkyl thiol terminated $\mathrm{MV}^{2+}(\mathrm{MV}-\mathrm{SH})$ was synthesized as previously reported..$^{48}$

Due to poor solubility of $\mathrm{CB}[8]$ in water and its hygroscopic nature, the apparent molecular weight of the commercial powder and its actual concentration in aqueous solutions were determined for each batch using a simple and highly reproducible method described previously. ${ }^{49} \mathrm{CB}[8]$ was dissolved in MilliQ water by sonication at $80^{\circ} \mathrm{C}$ for 2 hrs. 
Equipment. Polymerase Chain Reaction (PCR) was performed using a Peqlab Primus 25 advanced thermocycler. Fast Protein Liquid Chromatography (FPLC) was performed using ÄKTApurifier (GE Healthcare Life Sciences) in combination with a Frac-950 fractionation collector (GE Healthcare Life Sciences). The used column was a Superdex 75 10/300GL (GE Healthcare Life Sciences) and had a separation range for molecules with molecular weights between 3000 and 70000 Da. UV-Vis measurements to determine DNA and protein concentrations were performed using a Thermo Scientific Nanodrop 1000. Matrix assisted laser desorption/ionisation time-of-flight (MALDI-TOF) analysis was performed with a Waters MALDI SYNAPT High Definition Mass Spectrometer. UV-Vis measurements for trypsin inhibitor assays were performed using a Perkin Elmer Victor $\mathrm{X}_{3}$ multiwell plate reader. Miscroscale thermophoresis measurements were performed using a Nanotemper Monolith NT.115 device. Surface plasmon resonance (SPR) experiments were conducted using $50 \mathrm{~nm}$ SPR gold substrates from Ssens bv on a Resonant-probes SPR. The reflectivity was measured at fixed angle at which point the linear region of the SPR curve stopped. An Olympus microscope $1 X_{71}$ with filters were used for recording fluorescent images.

Molecular cloning of knottin constructs. pET15b TFP-Ecoil vector was obtained from W. F. Rurup..$^{50}$ ssDNA sequences corresponding to the $\beta$-trypsin inhibitor knottin and primers to extend the $N$ and $C$ termini with the $C B[8]$-binding motif, GGWGG, were ordered from Eurofins MWG Operon, Germany. Amplification of the extended genetic construct was performed by PCR using pfu polymerase. The final genetic construct had 5' BsrGI and 3' Nhel restriction sites, which were used to insert it between the TFP and Ecoil in the plasmid through restriction digestion enzymes and T4 DNA ligase (New England Biolabs inc.). The 3' terminal also contained a stop codon to prevent expression of the Ecoil leucine zipper. The resulting PET15b TFP-K2 plasmid was transformed into NovaBlue ultracompetent cells (Novagen) and grown overnight on LB agar plates containing $100 \mathrm{mg} / \mathrm{L}$ ampicillin. Plasmids were extracted from individual colonies using Qiagen spin miniprep kit and the sequenced by Eurofins MWG Operon, Germany using a standard T7 terminal reverse primer. pET15b TFP-Ko, pET15b TFP-K1N and PET15b TFP-K1C plasmids were constructed by simultaneous site directed mutagenesis (QuickChange Lightning Multi Site-Directed Mutagenesis kit from Agilent Technologies) of the genetic sequences corresponding to both 5' and 3' GGWGG motifs to GGGGG. The mutated plasmids were transformed into XL10-Gold ultracompetent cells and grown overnight on LB agar plates containing $34 \mathrm{mg} / \mathrm{L}$ chloramphenicol and $100 \mathrm{mg} / \mathrm{L}$ ampicillin. Plasmids from several colonies were extracted, sequenced and bacterial cultures with each of the three different mutation combinations were 
identified. The four different plasmids were transformed into the expression host, Rosetta-Gami 2(DE3)pLysS competent cells (Novagen) and grown overnight on LB agar plates containing $34 \mathrm{mg} / \mathrm{L}$ chloramphenicol and 100mg/L ampicillin. Individual colonies were grown in LB media containing the mentioned antibiotics. For long term storage, $15 \%$-glycerol bacterial stocks were made and placed at $-80^{\circ} \mathrm{C}$.

\section{DNA sequences for PCR to construct K2}

Template: $\beta$-Trypsin inhibitor knottin + spacers

5'_TCAGGTTCAGGTTCAGGTCGCGTGTGCCCGCGCATTCTGATGGAATGCAAAAAAGATAGCG ATTGCCTGGCGGAATGCGTGTGCCTGGAACATGGCTATTGCGGCGGAAGTGGAAGTGGAAGT_ 3'

Fwd Primer: Filler-BsrGI-DDDDK-GGWGG-KnottinFwd

5'_agtgtaTGTACAatGATGACGACGACAAGGGAGGATGGGGCGGATCAGGTTCAGGT_3'

Rev Primer: Filler-Nhel-Stop-GGWGG-KnottinRev

5'_atcactGCTAGCTTAACCACCCCAACCACCACTTCCACTTCCACTTCC_3'

\section{Site directed muatagenesis primers}

Fwd1: 5'_ACGACGACAAGGGAGGAGGGGGCGGAT_3'

Fwd2: 5'_GTGGAAGTGGAAGTGGTGGTGGGGGTGGTTAA_3'

Protein expression and purification. $5 \mathrm{ml}$ bacterial starter cultures were grown overnight from glycerol stocks at $37^{\circ} \mathrm{C}$ with shaking in LB media containing appropriate antibiotics. This was then transferred into $1 \mathrm{~L}$ of the same media and the cultures were grown till they attained O.D.60onm values between 0.4 and 0.8 . Protein expression was then induced using isopropyl-B-D-1-thiogalactopyranoside (IPTG) at a final concentration of $0.1 \mathrm{mM}$. These cultures were grown overnight at $18^{\circ} \mathrm{C}$ with shaking. The cultures were then spun down at $6000 \mathrm{rcf}$ for $10 \mathrm{mins}$ at $4^{\circ} \mathrm{C}$ and supernatants were discarded. Bacterial pellets were resuspended in $10 \mathrm{~mL}$ BugBuster protein extraction reagent (Novagen) with $10 \mu \mathrm{L}$ Benzonase (30 $\mathrm{U} / \mu \mathrm{L}$, Novagen) and gently shaken for 20 mins at $25^{\circ} \mathrm{C}$. TFP-fused knottin constructs were then purified using HisSelect ${ }^{\odot}$ Nickel affinity columns (Sigma Aldrich) into an elution buffer of $50 \mathrm{mM}$ $\mathrm{NaH}_{2} \mathrm{PO}_{4} 300 \mathrm{mM} \mathrm{NaCl} 250 \mathrm{mM}$ imidazole pH8. The purified TFP-fused knottins were then rebuffered into $\mathrm{pH} 7.4$ phosphate buffered saline (PBS, Sigma Aldrich) using 30 kDa-cutoff centrifugal filter units (Amicon Ultra). Concentrations of these TFP-fused 
knottins were determined from the absorbance value at $467 \mathrm{~nm}$ and an extinction coefficient of $64000 \mathrm{M}^{-1} \mathrm{~cm}^{-1}$. Knottins were cleaved from the TFP using enterokinase enzyme (EKMax ${ }^{\mathrm{TM}}$, Life Technologies). The optimized reaction conditions required 50 ng of protein per $30 \mu \mathrm{L}$ reaction with 0.1 units of enterokinase in $\mathrm{pH}=7.5$ buffer containing $20 \mathrm{mM}$ tris $\mathrm{HCl}, 10 \mathrm{mM} \mathrm{NaCl}, 2 \mathrm{mM} \mathrm{CaCl}$ at $37^{\circ} \mathrm{C}$ for 16 hrs. The cleaved knottin was isolated from the other proteins using a 10kDa-cutoff centrifugal filter unit (Amicon Ultra) followed by a $3 \mathrm{kDa}$-cutoff centrifugal filter unit (Amicon Ultra). Final knottin solutions were in PBS. Concentrations of the knottin constructs were determined from the absorbance value at $280 \mathrm{~nm}$ and extinction coefficients of 12865 $\mathrm{M}^{-1} \mathrm{~cm}^{-1}$ for K2, $7365 \mathrm{M}^{-1} \mathrm{~cm}^{-1}$ for K1N and $\mathrm{K}_{1} \mathrm{C}$ and $1865 \mathrm{M}^{-1} \mathrm{~cm}^{-1}$ for Ko.

Amino acid sequence of $\mathrm{His}_{6}-$ TFP-K2

MGSSHHHHHHSSGLVPRGSHMVSKGEETTMGVIKPDMKIKLKMEGNVNGHAFVIEGEGEGKP YDGTNTINLEVKEGAPLPFSYDILTTAFAYGNRAFTKYPDDIPNYFKQSFPEGYSWERTMTFEDK GIVKVKSDISMEEDSFIYEIHLKGENFPPNGPVMQKKTTGWDASTERMYVRDGVLKGDVKHKLL LEGGGHHRVDFKTIYRAKKAVKLPDYHFVDHRIEILNHDKDYNKVTVYESAVARNSTDGMDELY NDDDDKGGWGGSGSGSGRVCPRILMECKKDSDCLAECVCLEHGYCGGSGSGSGGWGG

Blue = Hexa-His Tag, Gray $=$ Thrombin cleavage site, Dark Green $=$ TFP, Orange $=$ Enterokinase cleavage site, Red $=\mathrm{CB}[8]$-binding motif, Brown $=(\mathrm{SG})_{3} /(\mathrm{GS})_{3}$, Purple $=\mathrm{K}$

SDS-PAGE. SDS-PAGE was performed using a stacking gel with $6 \%$ crosslinking density and a resolving gel with $12 \%$ crosslinking density. After loading the prepared protein samples, $120 \mathrm{~V}$ potential was applied across the gel for approximately 90 mins. The gels was then rinsed well with water and stained with Bio-Safe Coomassie staining solution (Bio-Rad). 


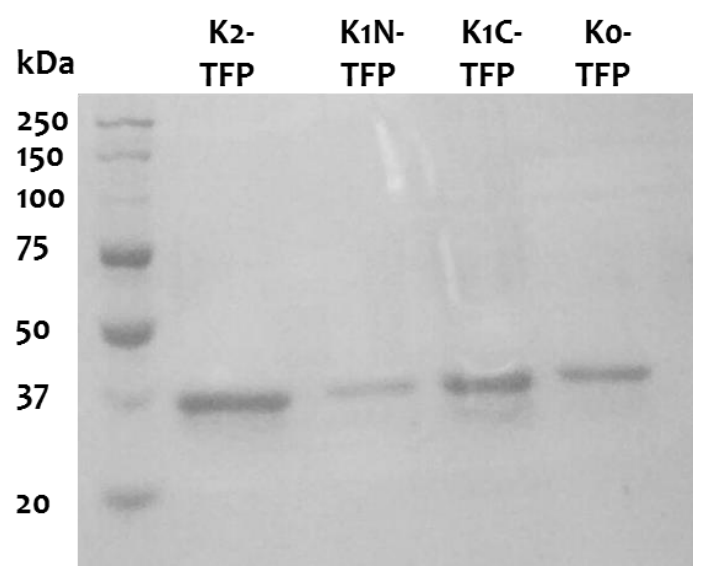

Figure 3.7. SDS-PAGE gel with bands corresponding to the different TFP-fused knottin constructs. Precision Plus Protein ${ }^{T M}$ Unstained Standards (Bio-Rad) was used as the reference for molecular weights.

FPLC and MALDI-TOF. During purification with the FPLC setup, the $\mathrm{pH}$, pressure, conductivity and the absorption at 280 and $467 \mathrm{~nm}$ were measured continuously. The flow speed of the buffer trough the column during purification was $0.7 \mathrm{ml} / \mathrm{min}$. The running buffer contained $50 \mathrm{mM}$ phosphate and $150 \mathrm{mM} \mathrm{NaCl}$ at $\mathrm{pH}$ 7.2. The injected volumes were 500uL.

For MALDI-TOF analysis, protein samples in water/buffer were mixed with equal proportions of acetonitrile $+0.1 \%$ TFA. Samples were prepared at a final protein concentration of $30 \mu \mathrm{M}$ and this was mixed with sinapinic acid (Sigma)-matrix. These were then measured with linear positive mode $\left(\mathrm{LD}^{+}\right)$.

The theoretical molecular weights of the miniproteins were determined using the ExPASy ProtParam tool (http://web.expasy.org/protparam/) and took into account the mass difference when 6 cysteines form 3 disulphide bridges $(\sim 6 \mathrm{~g} / \mathrm{mol})$. The matching of the theoretical and experimental values not only indicates that the protein sequence is correct but also verifies the formation of all disulphide bridges. 
a) FPLC
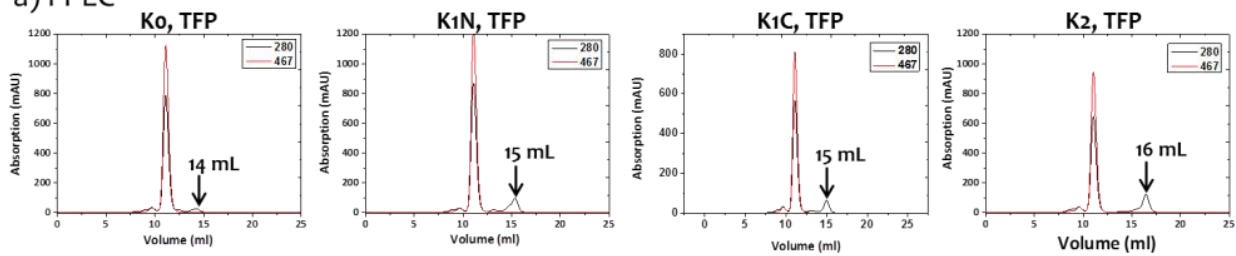

b) MALDI-TOF
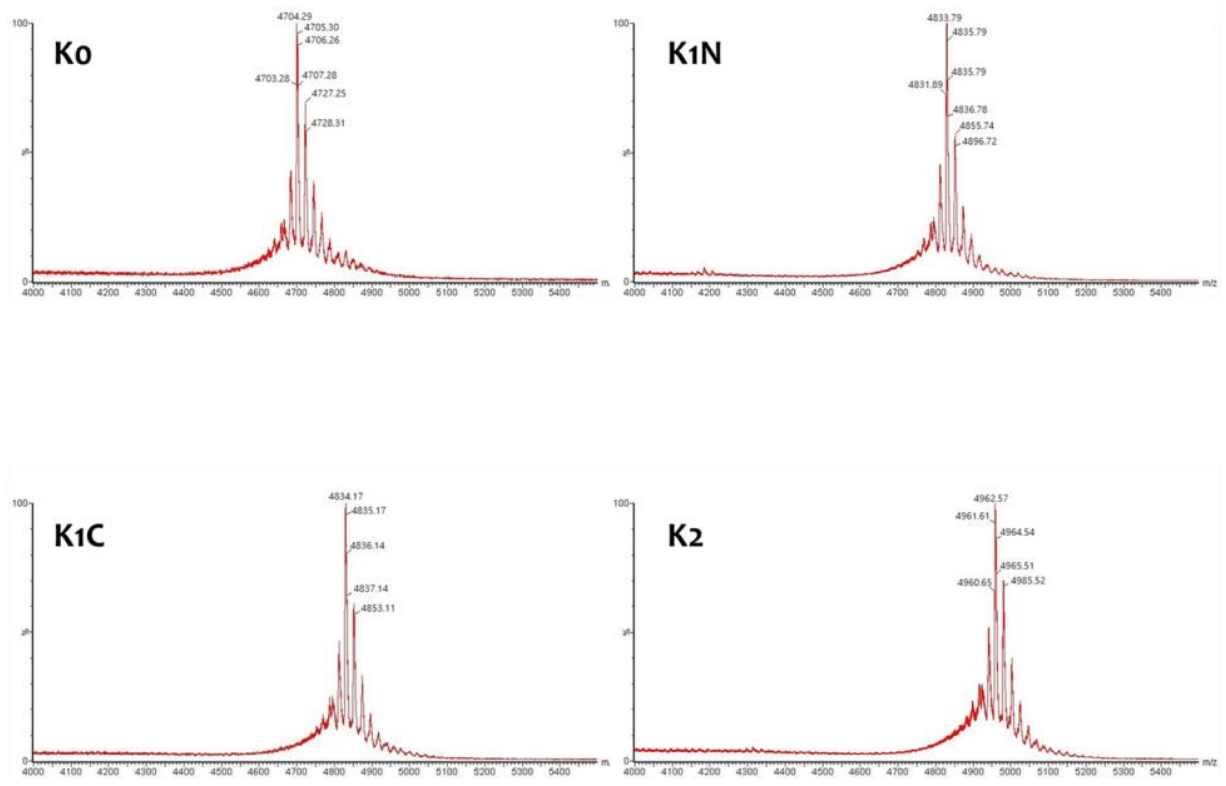

c) MALDI-TOF masses

\begin{tabular}{|l|l|l|}
\hline Knottin Construct & $\begin{array}{c}\text { Mass from MALDI-TOF } \\
(\mathrm{g} / \mathrm{mol})\end{array}$ & $\begin{array}{l}\text { Theoretical Molecular } \\
\text { Weight }(\mathrm{g} / \mathrm{mol})\end{array}$ \\
\hline K2 & 4962.57 & 4962.5 \\
\hline K1N & 4833.79 & 4833.3 \\
\hline K1C & 4834.17 & 4833.3 \\
\hline K0 & 4704.29 & 4704.1 \\
\hline
\end{tabular}

Figure 3.8. a) Size exclusion FPLC traces of the cleaved knottins still in the presence of TFP. Elution peaks corresponding to the different constructs seem to occur at slightly different elution volumes. b) MALDI-TOF results with peaks corresponding to the cleaved and purified knottin constructs. c) Mass values obtained from MALDI-TOF compared to theoretically calculated values.

Trypsin inhibitor assay. Trypsin cleaves peptides and proteins mainly at the carboxyl side of the amino acids lysine or arginine. During this experiment N-Benzoyl-L-Arginine Ethyl Ester (BAEE) was used as a substrate for trypsin digestion. This produces an 
absorbance shift from $230 \mathrm{~nm}$ to $253 \mathrm{~nm}$. The rate of reaction was determined for different concentrations of BAEE in the presence and absence of the knottins by following the increase in absorbance using a $260 \mathrm{~nm}$ filter. All measurements were performed in PBS.

Table 3.1. Vmax and $\mathrm{Km}$ values obtained from fitting the trypsin inhibitor assay data (Figure 3.3) using Michaelis-Menten equation (eq 3.1)

\begin{tabular}{|c|c|c|}
\hline Inhibitor & $\mathbf{V}_{\max }\left(\mathbf{m i n}^{-1}\right)$ & $\mathbf{K}_{\mathbf{m}}(\boldsymbol{\mu M})$ \\
\hline No inhibitor & $0.0058 \pm 0.0006$ & $206 \pm 40$ \\
\hline Ko-TFP & $0.0023 \pm 0.00007$ & $70 \pm 8$ \\
\hline K1N-TFP & $0.0022 \pm 0.00009$ & $71 \pm 11$ \\
\hline K1C-TFP & $0.0025 \pm 0.0001$ & $86 \pm 22$ \\
\hline K2-TFP & $0.0024 \pm 0.00004$ & $83 \pm 6$ \\
\hline Ko & $0.0017 \pm 0.00003$ & $58 \pm 4$ \\
\hline K1N & $0.00145 \pm 0.0002$ & $105 \pm 36$ \\
\hline K1C & $0.00165 \pm 0.00004$ & $47 \pm 4$ \\
\hline K2 & $0.00141 \pm 0.00006$ & $51 \pm 21$ \\
\hline
\end{tabular}

Microscale Thermophoresis. Solutions containing $1 \mu \mathrm{M}$ TFP-fused knottins, $150 \mu \mathrm{M} M V$ and concentrations of $\mathrm{CB}[8]$ ranging from $2 \mathrm{nM}$ to $37.5 \mu \mathrm{M}$ were made in PBS. Thermophoretic analyses were carried out in standard treated capillaries from Nanotemper using an LED power of $1 \%$ and MST power of $80 \%$ for $30 \mathrm{~s}$. Thermophoresis was calculated as the ratio of the fluorescence intensity after and before local heating.

Preparation of SAMs on Gold substrate. Gold substrates were first washed with piranha solution $\left(\mathrm{H}_{2} \mathrm{SO}_{4}+30 \% \mathrm{H}_{2} \mathrm{O}_{2}, \mathrm{v} / \mathrm{v} 3 / 1\right)$, copious amount of milli-Q water and finally with ethanol. Substrates were then immersed overnight in a $1 \mathrm{mM}$ ethanolic solution of $\left(E_{4} C_{11} S\right)_{2}$ and $M a l-E G_{6} C_{11}-S-S-C_{11}-E_{4}$ at a molar ratio of 99:1 at room temperature in the dark. The substrates were then cleaned thoroughly with ethanol, MilliQ water and dried with a stream of $\mathrm{N}_{2}$ gas. They were then immediately incubated with $1 \mathrm{mM} \mathrm{MV-SH}$ in $\mathrm{pH} 6.850 \mathrm{mM}$ phosphate buffer for $1 \mathrm{hr}$. The substrates were then washed thoroughly with MilliQ water, dried with $\mathrm{N}_{2}$ gas and used for further supramolecular assembly experiments.

Surface plasmon resonance. Surface adhesion measurements were carried out under conditions of constant flow $(100 \mu \mathrm{L} / \mathrm{min})$ using a pH 7.4 buffer of $0.5 \times$ PBS $+0.05 \%$ Tween20. All knottin solutions also contained $50 \mu \mathrm{M} \mathrm{CB}[8]$. 
Microcontact printing. Poly(dimethylsiloxane) (PDMS) stamps were prepared by casting a 10:1 (v/v) mixture of Sylgard 184 elastomer and curing agent (Dow Corning) against a patterned silicon master with $100 \mu \mathrm{m}$ circles having a depth of $30 \mu \mathrm{m}$. After curing the stamps at $60^{\circ} \mathrm{C}$ overnight, they were peeled off from the master before using. The individually cut out stamps were $\mathrm{O}_{2}$-plasma treated for 10 s to form a hydrophilic surface then inked with the appropriate solution for 20 mins after which they were dried with a gentle stream of $\mathrm{N}_{2}$ gas. These were then stamped on the $20 \mathrm{~nm}$ gold substrate with MV-SAMs for 20 mins with $15 \mathrm{~g}$ weight on top. The substrate was then briefly washed with MilliQ water and dried. In Figure 3.6a, $100 \mu \mathrm{M} \mathrm{CB}[8] / C B[7]$ in MilliQ water was printed followed by incubation of the substrate in $1 \mu \mathrm{M}$ TFP-fused knottin solution for 10 mins. The substrates were then briefly washed with MilliQ water, dried and imaged. In Figure 3.6b, a mixture of $50 \mu \mathrm{M} \mathrm{CB}[8]$ with $1 \mu \mathrm{M}$ TFP-fused knottin was printed for 10 mins followed by incubation in buffer containing PBS $+0.1 \%$ Tween 20 for 20 mins with mild shaking. The substrates were then washed with MilliQ water, dried and imaged. In Figure 3.6c, the previous strategy was followed using cleaved knottin solutions and after the incubation in buffer, the substrates were incubated in a $1 \mu \mathrm{M}$ Tryp-Cy3 solution for 10 mins then washed with MilliQ water, dried and imaged. All proteins including knottin constructs and Tryp-Cy3 were in $\mathrm{pH} 7.4$ buffer containing 0.5 $x$ PBS + 0.05\% Tween 20 .

\subsection{References}

(1) Kolmar, H. (2008) Alternative binding proteins: Biological activity and therapeutic potential of cystine-knot miniproteins. FEBS J. 275, 2684-2690.

(2) Austin, J., Wang, W., Puttamadappa, S., Shekhtman, A., and Camarero, J. A. (2009) Biosynthesis and biological screening of a genetically encoded library based on the cyclotide MCoTI-I. ChemBioChem 10, 2663-2670.

(3) Gelly, J., Gracy, J., Kaas, Q., Le-Nguyen, D., Heitz, A., and Chiche, L. (2004) The knottin website and database: a new information system dedicated to the knottin scaffold. Nucleic Acids Res. 32, D156-D159.

(4) Gracy, J., Le-Nguyen, D., Gelly, J.-C., Kaas, Q., Heitz, A., and Chiche, L. (2008) Knottin: the knottin or inhibitor cystine knot scaffold in 2007. Nucleic Acids Res. 36, D314-D319.

(5) Gould, A., Ji, Y., Aboye, T. L., and Camarero, J. A. (2011) Cyclotides, a novel ultrastable polypeptide scaffold for drug discovery. Curr. Pharm. Des. 17, 4294-4307.

(6) Moore, S. J., Leung, C. L., and Cochran, J. R. (2012) Knottins: disulfide-bonded therapeutic and diagnostic peptides. Pept. Modif. Pept. Drug Mol. 9, e3-e11.

(7) Banta, S., Dooley, K., and Shur, O. (2013) Replacing antibodies: engineering new binding proteins. Annu. Rev. Biomed. Eng. 15, 93-113.

(8) Hosse, R. J., Rothe, A., and Power, B. E. (2006) A new generation of protein display scaffolds for molecular recognition. Protein Sci. Publ. Protein Soc. 15, 14-27.

(9) Getz, J. A., Rice, J. J., and Daugherty, P. S. (2011) Protease-resistant peptide ligands from a knottin scaffold library. ACS Chem. Biol. 6, 837-844. 
(10) Getz, J. A., Cheneval, O., Craik, D. J., and Daugherty, P. S. (2013) Design of a cyclotide antagonist of neuropilin-1 and -2 that potently inhibits endothelial cell migration. ACS Chem. Biol. 8, 1147-1154.

(11) Heitz, A., Le-Nguyen, D., and Chiche, L. (1999) Min-21 and min-23, the smallest peptides that fold like a cystine-stabilized $\beta$-sheet motif: design, solution structure, and thermal stability. Biochemistry (Mosc.) 38, 10615-10625.

(12) Souriau, C., Chiche, L., Irving, R., and Hudson, P. (2005) New binding specificities derived from min-23, a small cystine-stabilized peptidic scaffold. Biochemistry (Mosc.) 44, 7143-7155.

(13) Chang, H.-J., Hsu, H.-J., Chang, C.-F., Peng, H.-P., Sun, Y.-K., Yu, H.-M., Shih, H.-C., Song, C.-Y., Lin, Y.-T., Chen, C.-C., Wang, C.-H., and Yang, A.-S. (2009) Molecular evolution of cystine-stabilized miniproteins as stable proteinaceous binders. Structure 17, 620-631.

(14) Huang, Y.-J., Chen, I.-C., Yu, C.-M., Lee, Y.-C., Hsu, H.-J., Ching, A. T. C., Chang, H.-J., and Yang, A.-S. (2010) Engineering anti-vascular endothelial growth factor single chain disulfide-stabilized antibody variable fragments (sc-dsFv) with phage-displayed sc-dsFv libraries. J. Biol. Chem. 285, 7880-7891.

(15) Silverman, A. P., Levin, A. M., Lahti, J. L., and Cochran, J. R. (2009) Engineered cystine-knot peptides that bind $a v \beta 3$ integrin with antibody-like affinities. J. Mol. Biol. 385, 1064-1075.

(16) Kimura, R. H., Levin, A. M., Cochran, F. V., and Cochran, J. R. (2009) Engineered cystine knot peptides that bind $\alpha_{v} \beta_{3}$, $\alpha v \beta_{5}$, and $\alpha_{5} \beta_{1}$ integrins with low-nanomolar affinity. Proteins Struct. Funct. Bioinforma. 77, 359-369.

(17) Kim, J. W., Cochran, F. V., and Cochran, J. R. (2015) A chemically cross-linked knottin dimer binds integrins with picomolar affinity and inhibits tumor cell migration and proliferation. J. Am. Chem. Soc. 137, 6-9.

(18) Jagadish, K., Borra, R., Lacey, V., Majumder, S., Shekhtman, A., Wang, L., and Camarero, J. A. (2013) Expression of fluorescent cyclotides using protein trans-splicing for easy monitoring of cyclotide-protein interactions. Angew. Chem. Int. Ed. 52, 3126-3131.

(19) Kimura, R. H., Cheng, Z., Gambhir, S. S., and Cochran, J. R. (2009) Engineered knottin peptides: a new class of agents for imaging integrin expression in living subjects. Cancer Res. 69, $2435-2442$.

(20) Miao, Z., Ren, G., Liu, H., Kimura, R. H., Jiang, L., Cochran, J. R., Gambhir, S. S., and Cheng, Z. (2009) an engineered knottin peptide labeled with $18 \mathrm{~F}$ for PET imaging of integrin expression. Bioconjug. Chem. 20, 2342-2347.

(21) Cabanas-Danés, J., Rodrigues, E. D., Landman, E., van Weerd, J., van Blitterswijk, C., Verrips, T., Huskens, J., Karperien, M., and Jonkheijm, P. (2014) A supramolecular host-guest carrier system for growth factors employing VHH fragments. J. Am. Chem. Soc. 136, 12675-12681.

(22) Kehr, N. S., Atay, S., and Ergün, B. (2015) Self-assembled monolayers and nanocomposite hydrogels of functional nanomaterials for tissue engineering applications. Macromol. Biosci. 15, 445-463.

(23) Wu, G., Li, P., Feng, H., Zhang, X., and Chu, P. K. (2015) Engineering and functionalization of biomaterials via surface modification. J. Mater. Chem. B 3, 2024-2042.

(24) Elemans, J. A. A. W., Lei, S., and De Feyter, S. (2009) Molecular and supramolecular networks on surfaces: from two-dimensional crystal engineering to reactivity. Angew. Chem. Int. Ed. 48, $7298-7332$.

(25) Uhlenheuer, D. A., Petkau, K., and Brunsveld, L. (2010) Combining supramolecular chemistry with biology. Chem. Soc. Rev. 39, 2817-2826. 
(26) Brinkmann, J., Cavatorta, E., Sankaran, S., Schmidt, B., van Weerd, J., and Jonkheijm, P. (2014) About supramolecular systems for dynamically probing cells. Chem. Soc. Rev. 43, 44494469.

(27) Ma, X., and Zhao, Y. (2014) Biomedical applications of supramolecular systems based on host-guest interactions. Chem. Rev. (Online early access) DOI: 10.1021/cr500392W.

(28) Boekhoven, J., and Stupp, S. I. (2014) 25th anniversary article: Supramolecular materials for regenerative medicine. Adv. Mater. 26, 1642-1659.

(29) An, Q., Brinkmann, J., Huskens, J., Krabbenborg, S., de Boer, J., and Jonkheijm, P. (2012) A supramolecular system for the electrochemically controlled release of cells. Angew. Chem. Int. Ed. 51, 12233-12237.

(30) Boekhoven, J., Rubert Pérez, C. M., Sur, S., Worthy, A., and Stupp, S. I. (2013) Dynamic display of bioactivity through host-guest chemistry. Angew. Chem. Int. Ed. 52, 12077-12080.

(31) Voskuhl, J., Sankaran, S., and Jonkheijm, P. (2014) Optical control over bioactive ligands at supramolecular surfaces. Chem. Commun. 50, 15144-15147.

(32) Sankaran, S., Kiren, M. C., and Jonkheijm, P. (2015) Incorporating bacteria as a living component in supramolecular self-assembled monolayers through dynamic nanoscale interactions. ACS Nano.

(33) Masson, E., Ling, X., Joseph, R., Kyeremeh-Mensah, L., and Lu, X. (2012) Cucurbituril chemistry: a tale of supramolecular success. RSC Adv. 2, 1213-1247.

(34) Lee, J. W., Samal, S., Selvapalam, N., Kim, H.-J., and Kim, K. (2003) Cucurbituril homologues and derivatives: New opportunities in supramolecular chemistry. Acc. Chem. Res. 36, 621-630.

(35) Liu, S., Zavalij, P. Y., and Isaacs, L. (2005) Cucurbit[10]uril. J. Am. Chem. Soc. 127, 16798-16799.

(36) Lagona, J., Mukhopadhyay, P., Chakrabarti, S., and Isaacs, L. (2005) The cucurbit[n]uril family. Angew. Chem. Int. Ed. 44, 4844-4870.

(37) Stoffelen, C., Voskuhl, J., Jonkheijm, P., and Huskens, J. (2014) Dual stimuli-responsive selfassembled supramolecular nanoparticles. Angew. Chem. Int. Ed. 53, 3400-3404.

(38) Hu, C., Lan, Y., Tian, F., West, K. R., and Scherman, O. A. (2014) Facile method for preparing surface-mounted cucurbit[8]uril-based rotaxanes. Langmuir 30, 10926-10932.

(39) González-Campo, A., Brasch, M., Uhlenheuer, D. A., Gómez-Casado, A., Yang, L., Brunsveld, L., Huskens, J., and Jonkheijm, P. (2012) Supramolecularly oriented immobilization of proteins using cucurbit[8]uril. Langmuir 28, 16364-16371.

(40) Brinkmann, Jenny, Sankaran, Shrikrishnan, Rinnen, Stefan, Arlinghaus, F. Heinrich, De Boer, Jan, and Jonkheijm Pascal. Achieving well defined reversible dynamic cell interfaces through supramolecular host-guest chemistry. Manuscript under preparation.

(41) Bush, M. E., Bouley, N. D., and Urbach, A. R. (2005) Charge-mediated recognition of nterminal tryptophan in aqueous solution by a synthetic host. J. Am. Chem. Soc. 127, 14511-14517.

(42) Schmoldt, H.-U., Wentzel, A., Becker, S., and Kolmar, H. (2005) A fusion protein system for the recombinant production of short disulfide bond rich cystine knot peptides using barnase as a purification handle. Protein Expr. Purif. 39, 82-89.

(43) Bode, W., Greyling, H. J., Huber, R., Otlewski, J., and Wilusz, T. (1989) The refined 2.0 A X-ray crystal structure of the complex formed between bovine beta-trypsin and CMTI-I, a trypsin inhibitor from squash seeds (Cucurbita maxima). Topological similarity of the squash seed inhibitors with the carboxypeptidase A inhibitor from potatoes. FEBS Lett. 242, 285-292.

(44) Morrison, J. F. (1969) Kinetics of the reversible inhibition of enzyme-catalysed reactions by tight-binding inhibitors. Biochim. Biophys. Acta BBA - Enzymol. 185, 269-286. 
(45) Uhlenheuer, D. A., Wasserberg, D., Haase, C., Nguyen, H. D., Schenkel, J. H., Huskens, J., Ravoo, B. J., Jonkheijm, P., and Brunsveld, L. (2012) Directed supramolecular surface assembly of SNAP-tag fusion proteins. Chem. - Eur. J. 18, 6788-6794.

(46) Nguyen, H. D., Dang, D. T., van Dongen, J. L. J., and Brunsveld, L. (2010) Protein dimerization induced by supramolecular interactions with cucurbit[8]uril. Angew. Chem. 122, 907-910.

(47) Voskuhl, J., Brinkmann, J., and Jonkheijm, P. (2014) Advances in contact printing technologies of carbohydrate, peptide and protein arrays. Arrays 18, 1-7.

(48) Tian, F., Cheng, N., Nouvel, N., Geng, J., and Scherman, O. A. (2010) Site-selective immobilization of colloids on au substrates via a noncovalent supramolecular "handcuff." Langmuir 26, 5323-5328.

(49) Yi, S., and Kaifer, A. E. (2011) Determination of the purity of cucurbit[n]uril $(n=7,8)$ host samples. J. Org. Chem. 76, 10275-10278.

(50) Rurup, W. F., Verbij, F., Koay, M. S. T., Blum, C., Subramaniam, V., and Cornelissen, J. J. L. M. (2014) Predicting the loading of virus-like particles with fluorescent proteins. Biomacromolecules $15,558-563$.

\subsection{Supporting information}

\subsubsection{Aggregation of cleaved knottins}

From the UV-Vis absorbance spectra of cleaved $\mathrm{K} 2$ knottin, it can be seen that at $3 \mu \mathrm{M}$, a well-defined peak is formed at $280 \mathrm{~nm}$ wavelength, whereas at $6 \mu \mathrm{M}$ this peak seems to have broadened. As expected, the $280 \mathrm{~nm}$ absorbance value is nearly double for the higher concentration ( $0.04 \mathrm{AU}$ for $3 \mu \mathrm{M}$ and 0.1 for $\sim 6 \mu \mathrm{M}$ ). However, beyond $320 \mathrm{~nm}$ absorbance of the $3 \mu \mathrm{M}$ sample drops to nearly zero while the absorbance of the higher concentration sample stays significantly higher than zero up to almost $600 \mathrm{~nm}$. This is usually caused by scattering of light by protein aggregates, resulting in absorbance even at higher wavelengths.

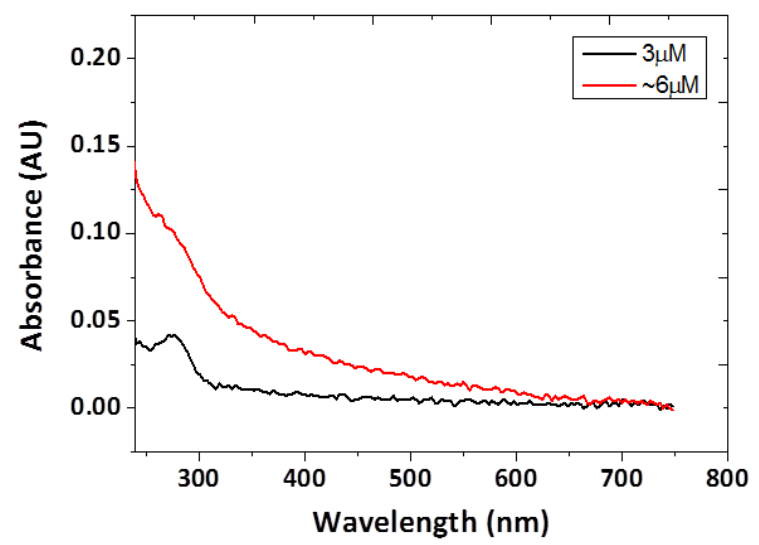

Figure S3.1. UV-Vis absorbance spectrum of cleaved K2 at two different concentrations. 


\subsubsection{SPR of TFP-fused knottins}

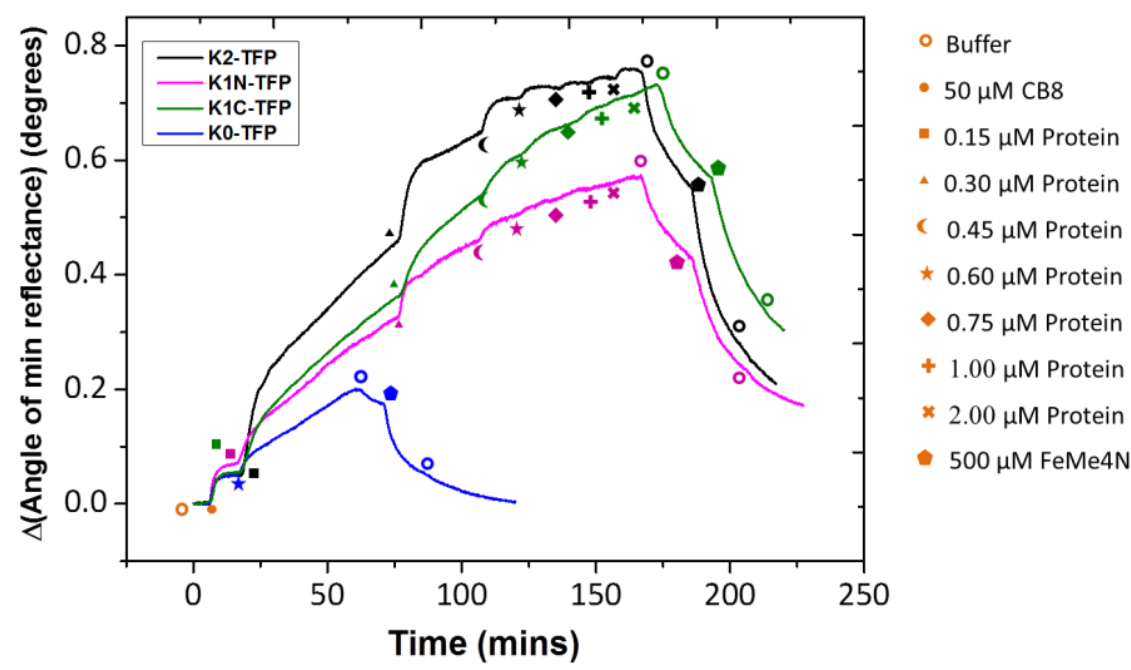

Figure S3.2. SPR titrations of different TFP-fused knottin constructs on $\mathrm{CB}[8]$ immobilized SAMs. The legend on the right lists the different solutions that were flowed over the substrate and the location of the symbols in the plot represent the time when they were introduced. Ferrocenylmethyl trimethylammonium (FeMe4N) is a high affinity $\mathrm{CB}[8]$-binding molecule used to cause competitive dissociation of $C B[8]$-bound proteins. All protein solutions also contained $50 \mu \mathrm{M} \mathrm{CB}[8]$ in them.

\subsubsection{SPR of $\beta$-trypsin surface adhesion}

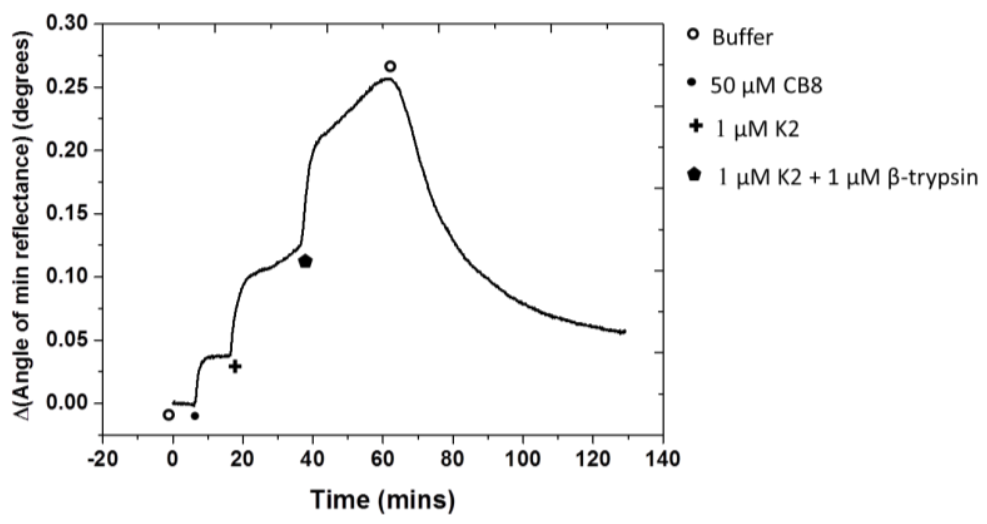

Figure S3.3. SPR response of B-trypsin association and dissociation on a $\mathrm{MV}^{2+}-\mathrm{CB}[8] \mathrm{SAM}$ displaying K2. The legend on the right lists the different solutions that were flowed over the substrate and the location of the symbols in the plot represent the time when they were introduced. All protein solutions also contained $50 \mu \mathrm{MCB}[8]$ in them. 


\section{Chapter 4}

\section{Scaffolding of cystine-stabilized miniproteins}

Biomolecular scaffolds were engineered by genetically fusing, in the form of chains, knottins and Min-23 constructs, which are classes of thermally and proteolytically stable miniproteins. By fusing these miniprotein chains to a teal fluorescent protein (TFP), an efficient strategy was devised for their production in E. coli. The binding properties of these scaffolds towards 6 -trypsin, VEGF and HIV-1 Nef proteins were analyzed with native ESI-mass spectrometry, microscale thermophoresis, surface plasmon resonance with imaging and a trypsin activity assay. Miniproteins within the chains were found to be functional and significantly robust. Various results indicated that they were able to bind multiple target proteins in a potentially multi-specific manner. Differences in binding and inhibition of 6 -trypsin by monovalent and bivalent miniprotein chains were clearly observed however similar trends were not as evident for VEGF and HIV-1 Nef. Issues dealing with folding, miniprotein orientations, non-specific adhesion and slow proteolytic degradation of the miniproteins have been speculated, which need to be carefully investigated and optimized before applications of these types of miniprotein chains can be undertaken. Once this is done, such constructs have the potential to be used in a very versatile manner for various molecular engineering applications.

SPR-i measurements done along with Ivan Stojanovic from the Medical Cell Biophysics group of the University of Twente

Native ESI-MS measurements were performed along with Arjan Barendregt and Prof. Dr. Albert J.R. Heck from the Biomolecular Mass Spectrometry and Proteomics group of the Utrecht University. 


\subsection{Introduction}

Engineered proteins with multiple epitopes that bind to either many copies of the same binding partner or with several different binding partners are emerging as powerful agents in molecular biology based fields. In therapeutics, protein-based drugs are highly sought after due to their biocompatibility, high affinity and natural specificity. ${ }^{1}$ Even though huge advances have been made in directed evolution and rational design algorithms to engineer proteins with higher affinities and better specificities, these traditional approaches still face concrete limitations. ${ }^{2}$ Multivalency and multispecificity provide new dimensions in macromolecular binding events to produce extremely effective proteinaceous drug candidates.3,4 They enable proteins to latch onto targets that are present only in particular densities, combinations and orientations. This allows the possibility of developing proteins that can distinguish between targets from healthy and infectious cells. ${ }^{5-7}$ Cochran and co-workers have worked extensively on developing such proteins that can simultaneously bind, for example, integrins with picomolar affinities ${ }^{8}$ or integrin and VEGFR2 receptors simultaneously. ${ }^{9}$ Design aspects and advantages of such proteins have further been detailed in a perspective article ${ }^{10}$ and book chapter ${ }^{11}$ written by them. Apart from therapeutics, multivalent and multispecific proteins are also being applied in metabolic engineering. ${ }^{12,13}$ Advances in the field have led to the possibility of using living organisms to produce molecular products that are used as drugs, cosmetics, biofuels etc. Synthetic biology has facilitated the expansion of the versatility of this technology, by introducing heterologous enzymes into organisms to make them synthesize chemicals that they would not naturally produce. ${ }^{14}$ An enzyme cascade consists of homologous and heterologous enzymes that work sequentially to convert reactants into products. This process involves several intermediates that need to travel from one enzyme to the next before the final product is obtained. When the enzymes are freely distributed, these intermediates have the possibility to diffuse away, interfere with the host organism's metabolism or get trapped within certain organelles resulting in poor production yields. Arranging the enzymes in close proximity of each other has been shown to solve such issues. ${ }^{12}$ One approach to achieve this is by genetically fusing together individual proteins bearing appropriate binding domains. ${ }^{12,13}$ Thus such multivalent and multi-specific protein constructs can act as scaffolds for the cascade enzymes.

The design and productions of such multivalent and multi-specific protein constructs has been achieved by various strategies. Individual proteins have been linked together with a chemical linker ${ }^{8}$ or by genetic fusion, ${ }^{13}$ binding sites have been modified to have 
multiple specificities ${ }^{15}$ and a second binding motif has been engineered into a protein with a different target. ${ }^{9}$ Amongst these approaches, the genetic fusion strategy seems to be most versatile and relatively simple. Fusion is done at the gene level, so any natural, synthetic or randomized protein sequence can be used. Binding motifs can be arranged in well-defined linear order. Linkers between proteins are usually composed of peptide sequences that can be engineered with necessary properties. Peptide tags can be introduced to aid in purification. It is possible to use these protein constructs in vivo, since they are produced by the organism in the final required form. However, this strategy also suffers from certain limitations. The protein constructs are usually susceptible to proteolytic degradation and might be sensitive to factors like temperature and $\mathrm{pH}$. Recombinant production of very large protein constructs is difficult in the most widely used expression hosts such as bacteria and yeast. Also, certain proteins require chaperones that exist only in the host organism for proper folding.

To address these issues, we sought after a family of proteins that are robust, small, autonomously folding and bind a large variety of targets. A family of miniproteins named knottins was found to fit these criteria. They are one of the smallest naturallyoccurring miniproteins (typically between 25-35 amino acids) and their threedimensional structure is essentially defined by a distinctive arrangement of three disulfide bonds. They are made up of a small triple-stranded antiparallel $\beta$-sheet, which is stabilized by the disulfide bond framework. ${ }^{16}$ Due to this structure, knottins are extremely robust with high melting temperatures $\left(T_{m}>100^{\circ} \mathrm{C}\right)$ and even resistant against proteolytic enzymes. Naturally occurring knottins have little sequence homology except the set of Cys residues, which gives rise to the conserved pattern of disulfide bridges. ${ }^{16}$ The interspersed peptide loops are highly variable both in length and sequence and consequently, there are several naturally occurring knottins that bind a wide variety of proteins. They have affinities towards their target proteins in the $\mathrm{nM}$ range and are found in nature as potent neurotoxins in spider and scorpion venom, insecticides produced by plants, protease inhibitors, antimicrobial agents etc. Due to their biocompatibility, several of these naturally occurring knottins have also been tested as possible analgesics, anti-malarials and pesticides. ${ }^{17}$ Exhaustive information about knottins, their structures and functions is available on an open-access online knottin database (http://knottin.cbs.cnrs.fr), created by Gracy and co-workers. ${ }^{18}$

As the peptide loops are amenable to modification, the core of the knottin structure has been exploited as a suitable scaffold to create interfaces for novel binding 
activities. ${ }^{16,19}$ Ever since the recombinant expression of knottins in E.coli was established, studies showed that individual peptide loops could be extensively modified with alternate sequences even up to 17 amino acids in length without diminishing the stability of the structure. ${ }^{20}$ Such knottins also have the remarkable ability to refold and correctly close its three disulfide bonds. ${ }^{19}$ This meant that knottins with unnatural binding motifs could be engineered by replacing the loops with peptide sequences of interest. ${ }^{21}$ Using bacterial, phage and yeast display techniques, knottins with randomized peptide loops were used to identify high affinity binders against proteins like thrombin, ${ }^{22}$ neuropilin ${ }^{23}$ and integrins. ${ }^{24}$ Furthermore, a truncated form of these naturally occurring knottins containing only 23 amino acids, called Min-23, has been engineered with only 2 disulfide bridges. ${ }^{25}$ Similar to its parent knottin, it is comprised of a cystine-stabilized $\beta$-sheet (CSB) motif that forms an autonomous folding unit with three $\beta$-strands and a short $\alpha$-helix. Even though Min-23 miniproteins are stabilized by only two disulfide bridges they still retain high melting temperatures $\left(T_{m}\right.$ $100^{\circ} \mathrm{C}$ ) and proteolytic resistance. The peptide sequence of the second $\beta$-turn is admissible to extensive modifications, up to 10 amino acids, using which high affinity binders have been identified for various target proteins like VEGF, HIV-Nef, mitochrondiral membrane protein TOM70 etc. ${ }^{26,27}$ These studies show that apart from their natural targets, knottins are also being extensively engineered to bind several other medically relevant target proteins.

Due to the mentioned properties of small size, autonomous folding, robustness and versatility in binding targets, we chose knottins and Min-23 as our prime candidates to attempt the construction of multivalent and multi-specific protein scaffolds. Since the binding domains are present on the loops, it would also be possible to genetically fuse their $\mathrm{N}$ and $\mathrm{C}$ terminals together to form a chain. To test our strategy, we selected three relatively small protein targets, $\beta$-trypsin, ${ }^{28}$ VEGF $^{29}$ and HIV-1 Nef. ${ }^{26} \beta$-trypsin is inhibited by a naturally occurring CMTI-I knottin from the winter squash plant. ${ }^{28}$ VEGF165 and HIV-1 Nef are bound by synthetic Min-23 derivatives identified by phage display techniques. ${ }^{26,29}$ Protein scaffolds were constructed by genetically fusing these three miniproteins ( $\beta$-trypsin inhibitor knottin - ' $B$ ', ${ }^{28}$ VEGF binding Min-23 - ' $V$ '29 and HIV-1 Nef binding Min-23 - ' $\mathrm{H}^{\prime 26}$ ) in the form of a chain, which was also fused to a teal fluorescent protein (TFP) to facilitate expression, purification and analysis (Figure 4.1). Four such fusion proteins were produced with the three miniproteins arranged in different combinations - BHV, BVB, HBH, VHV (Figure 4.1b). Their multivalency and multispecificity were evaluated using enzyme inhibitor assays, microscale 
thermophoresis (MST), mass spectrometry (MS), and surface plasmon resonance imaging (SPRi) measurements.
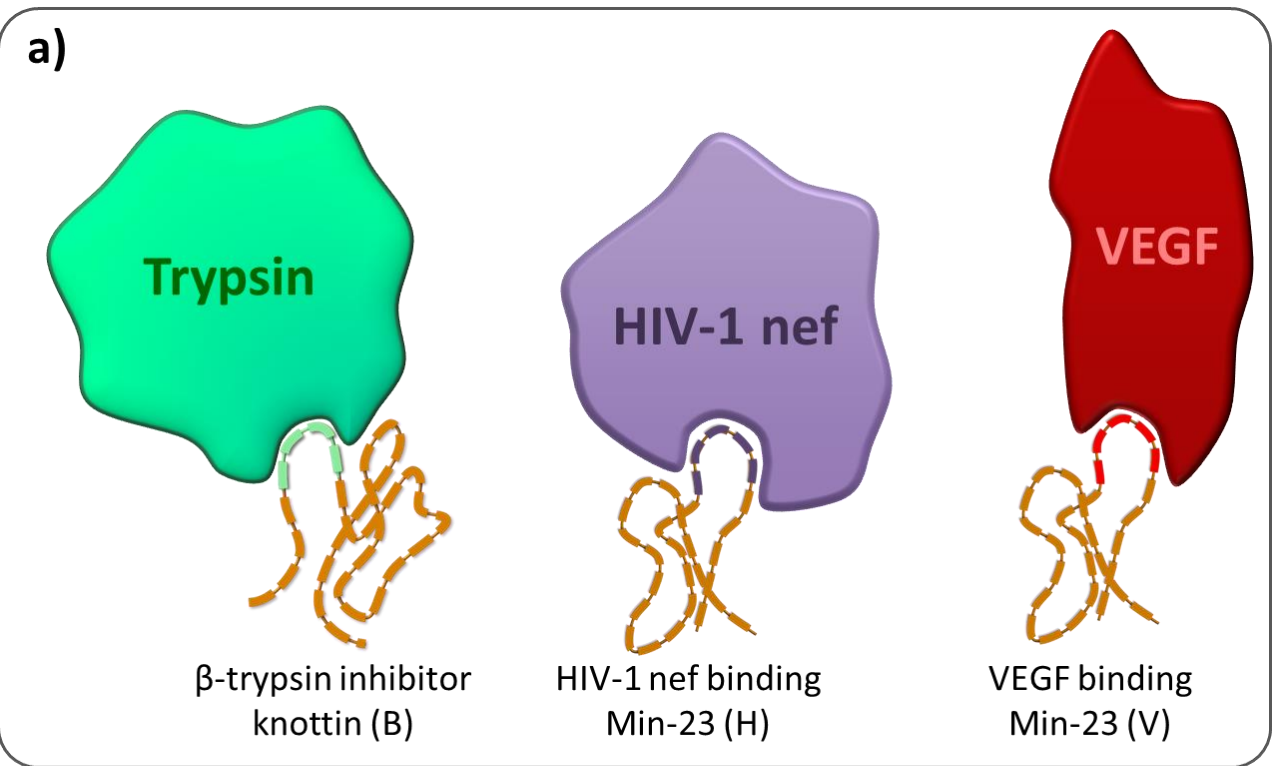

b)
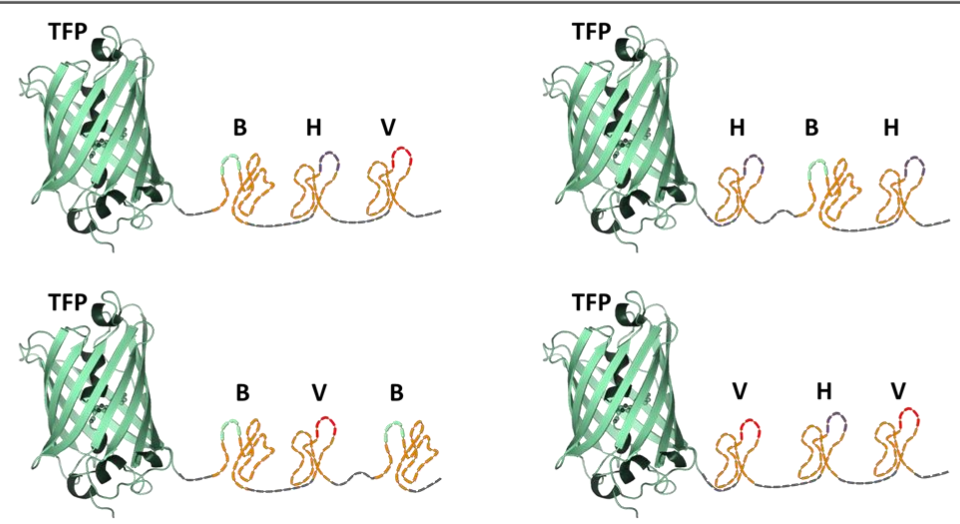

Figure 4.1. a) Graphical representation of the three miniproteins and corresponding target proteins used in this chapter. b) Schematic of the four different TFP-fused protein scaffolds constructed. 


\subsection{Results and Discussions}

\subsubsection{Design and recombinant synthesis strategy}

In order to efficiently construct the four different TFP-fused chains of miniproteins, standard recombinant DNA techniques were used with interchangeable components as displayed briefly in Figure 4.2a. The genes of each miniprotein were ordered with the same 5' and 3' flanking DNA sequences encoding for the peptide sequences of (SG) 3 at the $\mathrm{N}$ terminal and $(\mathrm{CS})_{3}$ at the $\mathrm{C}$ terminal. These miniprotein genes were amplified by polymerase chain reaction ( $P C R$ ) using primer sequences composed of the appropriate complementary DNA sequence for hybridization, a sequence encoding for $(A P)_{2}$ and finally the appropriate restriction site. All miniprotein DNA sequences were amplified with these components and the restriction sites dictated the location of the miniproteins in the chain. Restriction digestion was done individually for each miniprotein and also the expression plasmid containing the TFP gene. Ligation was done in one-pot with the appropriate components mixed together. The miniprotein chains were fused with TFP for three major purposes. First, knottins are poorly soluble and form inclusion bodies during expression in E.coli. TFP improves the solubility of the entire protein allowing for simpler purification and higher yields during expression. Second, TFP allows us to visualize the presence of the protein in all stages of expression and purification and enables us to easily determine its concentration. Finally, it acts as a fluorescent label for analysis techniques such as MST. Due to the presence of multiple disulfide bonds in the miniprotein chains, a special bacterial strain with a cytoplasm that favours disulfide bond formation, commercially available as Rosetta Gami 2, was used. In the final protein constructs, the miniproteins were separated by a peptide linker with the sequence GSGSGSAPAPXXPAPASGSGSG, where $X X$ represents variable amino acids encoded by the restriction sites. The maximum possible distance of this linker would span $7.7 \mathrm{~nm}$, which should provide sufficient space for the binding of the target proteins (The diameter of trypsin and HIV-1 Nef is $\sim 4$ $\mathrm{nm}^{28,30}$ while the VEGF dimer has a length of $\sim 6.5 \mathrm{~nm}$ and width of $\sim 4 \mathrm{~nm}^{31}$ ). The linker residues have also been selected such that they are not easily susceptible to proteolytic enzymes. ${ }^{32}$ The $(\mathrm{SG})_{3} /(\mathrm{GS})_{3}$ repeat regions are flexible to allow several orientations for protein binding and the $(P A)_{2} /(A P)_{2}$ regions serve as rigid spacers to ensure that the miniproteins remain separated. 33

The proteins also had an $\mathrm{N}$-terminal hexa-His tag for facile purification with metal affinity columns. After all the purification steps, we obtained an average of $20 \mathrm{mg} / \mathrm{L}$ of the TFP-fused miniprotein chain protein constructs, which corresponds to an average 
of $6.5 \mathrm{mg} / \mathrm{L}$ of just the miniprotein chains and is comparable to the production yields of individual knottins seen in Chapter 3 and in literature. ${ }^{34}$ The protein sizes were characterized using SDS-PAGE (Figure 4.2b) and ESI-MS (Figure 4.2C) and were found to be agreeable with the theoretically expected values.
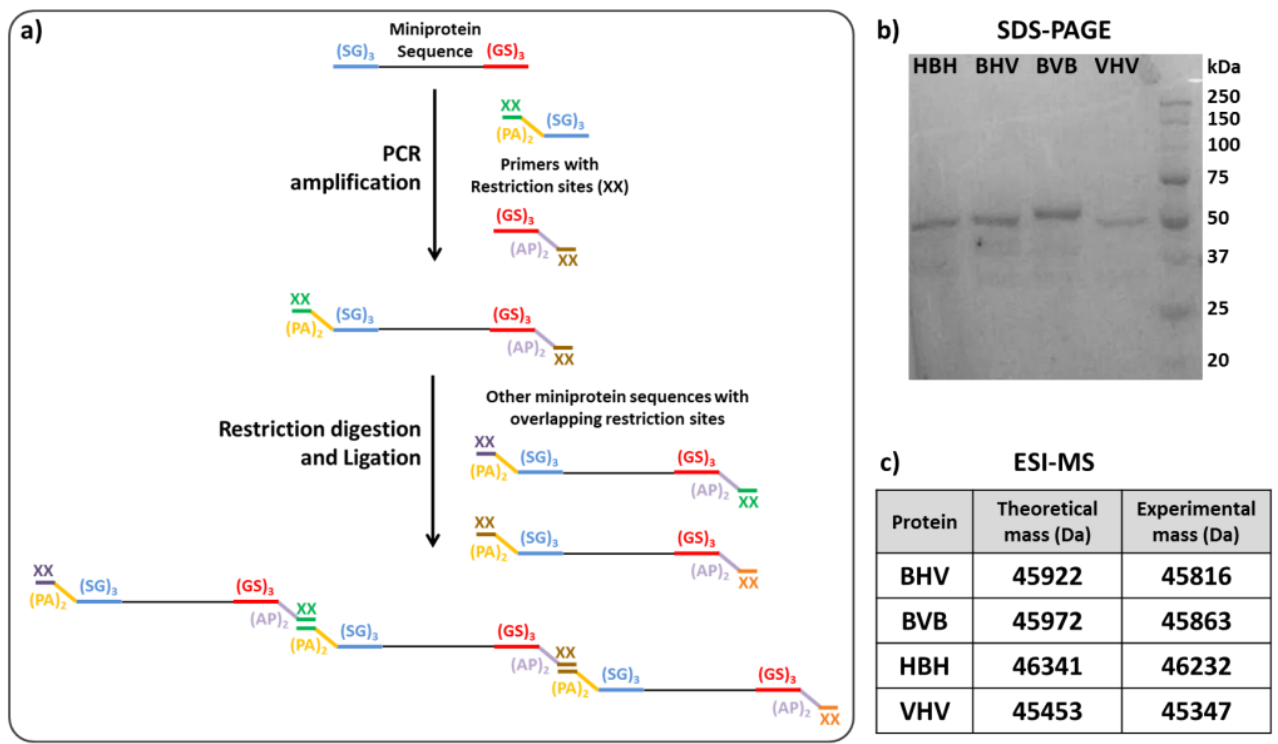

Figure 4.2. a) Schematic elaborating the recombinant strategy employed to genetically construct the miniprotein chain fusion proteins. The lines are symbolic of the DNA sequence and the letters are the encoded amino acids. XX represents two variable amino acids encoded by restriction sites. $b$ ) SDS-PAGE gel with the four different TFP-fused miniprotein chain constructs along with a Precision Plus Protein ${ }^{T M}$ Unstained Standards ladder. c) Masses of the four TFP-fused miniprotein chain constructs determined by ESI-MS compared to theoretical values. MS plots are provided in Figures $4.7-4.10$ (see in the Experimental section).

\subsubsection{Trypsin inhibition analysis}

Since the enzymatic activity of $\beta$-trypsin can be assayed, its inhibition by $\mathbf{B}$ would prove that the knottin is correctly folded and functional when incorporated in the miniprotein chain. $\beta$-trypsin's proteolytic activity was monitored using Na-Benzoyl-L-arginine ethyl ester (BAEE). This molecule gets cleaved and the product has a red-shifted absorbance peak at $253 \mathrm{~nm}$ wavelength. When BAEE was titrated with a fixed concentration of trypsin in presence and absence of the four miniprotein chain constructs, it was clearly seen that the rate of the reaction dropped in the presence of the miniprotein chains containing B (Figure 4.3a). At all concentrations, the reaction rates in the presence of 
VHV were similar to those with only trypsin, confirming that inhibition occurred only due to the presence of $\mathbf{B}$ in the other miniprotein chain constructs. BVB clearly exhibited the highest level of inhibition indicating that both the $\mathbf{B}$ knottins were available to interact with $\beta$-trypsin. All plots were fitted with the Michaelis-Menten equation (eq 4.1) to determine the asymptotic maximum reaction rate, $V_{\max }$ and the Michaelis constant, $K_{m}$.

$$
v=\frac{V_{\max }[S]}{K_{m}+[S]} \quad \text { eq } 4.1
$$

From Table 4.1, it can be seen that $V_{\max }$ values of the inhibited reactions are lower than that of the uninhibited reaction and the $K_{m}$ values vary only slightly. Also, the inhibitor concentrations $(100 \mathrm{nM})$ are several orders lower than the $K_{m}$ values $(50-150 \mu \mathrm{M})$. These indicate that pseudo-irreversible inhibition is occurring due to very strong inhibition as has been described in Chapter 3, section 3.2.2. Using eq 4.2, the apparent inhibitor constants $K_{\text {iapp }}$ values were determined.

$$
\frac{V_{i}}{V_{0}}=1-\frac{\left[E_{T}\right]+\left[I_{T}\right]+K_{i a p p}-\left\{\left(\left[E_{T}\right]+\left[I_{T}\right]+K_{i a p p}\right)^{2}-4\left[E_{T}\right]\left[I_{T}\right]\right\}^{1 / 2}}{2\left[E_{T}\right]} \quad \text { eq } 4.2
$$

where $V_{i}$ and $V_{0}$ are the $V_{\max }$ values of the inhibited and uninhibited reactions respectively, $\left[E_{T}\right]$ is the total concentration of $\beta$-trypsin, $\left[I_{T}\right]$ is the total knottin concentration and $K_{\text {iapp }}$ is the apparent dissociation constant of the enzyme-inhibitor complex.

This analysis provided $K_{\text {iapp }}$ values in the nanomolar range, similar to values obtained previously. Surprisingly, BVB provided a $K_{\text {iapp }}$ value $(6 \pm 1 \mathrm{nM})$ that was one order of magnitude lower than that of BHV and HBH $(\sim 60 \mathrm{nM})$. Even though BVB is expected to inhibit more efficiently, the Kiapp value would only be expected to be a half of that of $\mathbf{B H V}$ and $\mathbf{H B H}$ since the binding sites are expected to be independent. A more thorough determination of the $K_{\text {iapp }}$ values was performed by carrying out the trypsin inhibitor assay with various concentrations of the miniprotein chain constructs, keeping trypsin and BAEE concentrations constant (Figure 4.3b). All inhibited reaction rates were normalized as fractions of the uninhibited reaction rate. Fitting the resulting plots with eq 4.2 provided a BVB $K_{\text {iapp }}$ value of $27 \pm 2 \mathrm{nM}$, which was nearly half the value obtained for BHV $(74 \pm 8 \mathrm{nM})$ and $\mathbf{H B H}(86 \pm 10 \mathrm{nM})$. These results indicate that the $\mathbf{B}$ knottins had folded properly and were functional. Furthermore, improved inhibition of BVB suggested that the two $\mathbf{B}$ knottins in the chain were both free to interact with $\beta$ trypsin. 
a)

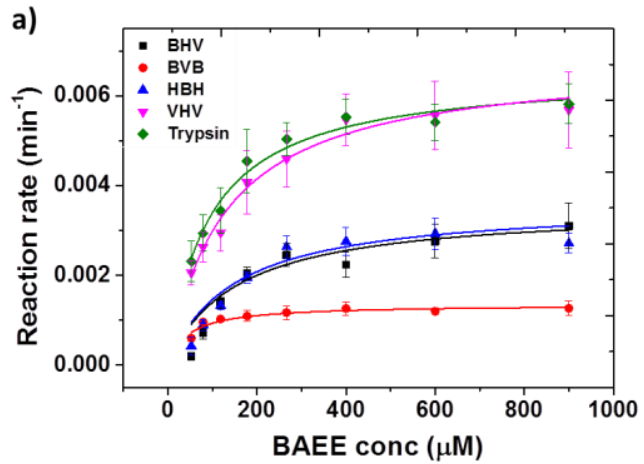

b)

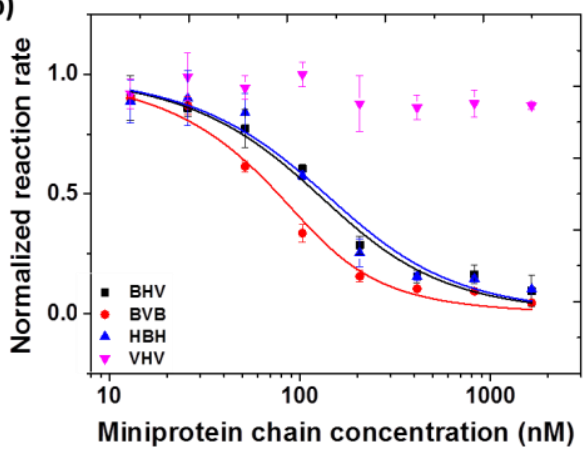

Figure 4.3. a) Reactions rates of the cleavage of various concentrations of BAEE by $100 \mathrm{nM}$ B-trypsin in the presence and absence of $100 \mathrm{nM}$ of the four miniprotein chains have been plotted against corresponding BAEE concentrations. Values are presented as mean $\pm S T E, n=3$. The solid lines represent fitting using the Michaelis-Menten equation (eq 4.1). The $K_{\text {iapp }}$ values obtained from eq 4.2 are $63 \pm 7 \mathrm{nM}$ for $\mathrm{BHV}, 6 \pm 1 \mathrm{nM}$ for $\mathrm{BVB}$ and $68 \pm 9 \mathrm{nM}$ for HBH. c) Reaction rates of cleavage of 400 $\mu M$ BAEE by $100 \mathrm{nM}$ 8-trypsin in the presence of various concentrations of the four miniprotein chains were normalized as a fraction of the reaction rate of the uninhibited reaction and plotted against the corresponding miniprotein chain concentrations. Values presented as mean \pm STE, $n=2$. The solid lines represent fitting using eq 4.2. The obtained $K_{\text {iapp }}$ are $74 \pm 8 \mathrm{nM}$ for $\mathbf{B H V}, 27 \pm 2 \mathrm{nM}$ for BVB and $86 \pm 10 \mathrm{nM}$ for $\mathbf{H B H}$.

Table 4.1. Michaelis-Menten fitting parameters from Figure 4.3a

\begin{tabular}{|l|c|c|}
\hline Protein & $V_{\max }\left(\mathrm{min}^{-1}\right)$ & $K_{m}(\mu \mathrm{M})$ \\
\hline Trypsin & $6.5 \times 10^{-3} \pm 0.2 \times 10^{-3}$ & $92 \pm 10$ \\
\hline BHV & $3.5 \times 10^{-3} \pm 0.7 \times 10^{-4}$ & $152 \pm 8$ \\
\hline BVB & $1.4 \times 10^{-3} \pm 0.8 \times 10^{-3}$ & $45 \pm 11$ \\
\hline HBH & $3.6 \times 10^{-3} \pm 0.5 \times 10^{-3}$ & $147 \pm 26$ \\
\hline VHV & $6.8 \times 10^{-3} \pm 0.3 \times 10^{-3}$ & $128 \pm 13$ \\
\hline
\end{tabular}

\subsubsection{MS analysis of protein binding to miniprotein chains}

Native ESI-MS was used to analyse the protein complexes formed between $\beta$-trypsin and the miniprotein chains. For this technique all proteins had to be rebuffered into an aqueous $150 \mathrm{mM}$ ammonium acetate buffer (AmAc) having $\mathrm{pH} 7.5$ since this volatile salt 
is known to produce very clean mass spectra. ${ }^{35}$ Relatively high protein concentrations of $>1 \mu \mathrm{M}$ were also required for good signal-to-noise ratios. Consequently, two strategies were attempted - one in which the proteins were rebuffered into AmAc and then mixed (Table 4.2), the other in which the proteins were mixed in PBS buffer and then the mixture was rebuffered into AmAc (Table 4.3). In either case, the proteins were allowed to interact for at least 10 mins before their masses were measured. In all cases it can be seen that part of the protein has been cleaved by $\beta$-trypsin. The remaining fragments, from start to end residues, detected in the spectra are indicated in brackets. $\beta$-trypsin is known to be a highly aggressive proteolytic enzyme that can cleave proteins at the C-terminal of arginine and lysine residues. All the TFP-fused miniprotein chains have an $\mathrm{N}$-terminal hexa-His Tag followed by a thrombin cleavage site (LVPRGS) with an arginine residue at the $17^{\text {th }}$ position. This is a sequence that is highly susceptible to proteolysis by $\beta$-trypsin, resulting in the $(18-$ end) fragments. This cleaved region does not affect the binding of the miniprotein chains and so all the (18 end) fragments can be considered as unaffected. When locating all the other cleaved residues in the protein sequences, they are found to be either at an arginine or lysine on the TFP or at the start of some miniproteins. The main core of the miniproteins and especially the binding loops seem to be unaffected, suggesting that they retain their resistance against proteolysis. Considering that at $\mu M$ concentrations $\beta$-trypsin has extremely high proteolytic activity combined with the fact that intact miniprotein chains were detected, this implies that the design of the miniprotein chains is highly robust. When the molar ratio of $\beta$-trypsin to miniprotein chain was 1:2, more intact TFPfused miniprotein chains were observed compared to when the ratio was 2:1. In the 1:1 molar ratio samples, where the proteins were mixed prior to buffer exchange, several cleaved fragments were also observed, which could be related to the fact that buffer exchange takes nearly 2 hrs allowing trypsin more time to act.

Complexes of $\beta$-trypsin with BHV, BVB and $\mathbf{H B H}$ were made in various ratios and observed in almost all cases, while no complexes of $\beta$-trypsin were detected with VHV. This confirmed that complexes were formed only when the B knottin was present. Even though a complex of BVB with two $\beta$-trypsins was not detected, it could be seen that cleavage of this TFP-fused miniprotein chain had occurred much less than the other constructs consisting of one B knottin, suggesting improved inhibition as observed in the trypsin inhibition experiments (Section 4.2.2.). We were also unable to observe complexes between VEGF or HIV-1 Nef with the miniprotein chains using this technique even though the concentrations for complex formation was high enough. 
This could possibly be due to an effect of the buffer or that the conditions used during these measurements were not optimal to observe these complexes.

Table 4.2. Native ESI-MS masses observed in solutions of 8-trypsin and miniprotein chains. These measurements were done by rebuffering the mixtures of proteins into aqueous AmAc. The mass sprectra are presented in Figures 4.6 - 4.10 (see in the Experimental Section)

\begin{tabular}{|c|c|c|c|c|}
\hline $\begin{array}{c}\text { Miniprotein } \\
\text { chain }\end{array}$ & $\begin{array}{c}\text { Miniprotein } \\
\text { chain conc. } \\
(\mu \mathrm{M})\end{array}$ & $\begin{array}{c}\text { Trypsin conc. } \\
(\mu \mathrm{M})\end{array}$ & $\begin{array}{c}\text { Masses } \\
\text { obtained (Da) }\end{array}$ & Mass assignment \\
\hline \multirow{8}{*}{ BHV } & \multirow{4}{*}{5} & \multirow{4}{*}{2.5} & 44041 & BHV (18 - end) \\
\hline & & & 67368 & BHV (18 - end) $+\beta$-Trypsin \\
\hline & & & 88164 & BHV (18 - end) dimer \\
\hline & & & 111449 & BHV (18 - end) dimer $+\beta$-Trypsin \\
\hline & \multirow{4}{*}{5} & \multirow{4}{*}{10} & 23293 & $\beta$-Trypsin \\
\hline & & & 27352 & BHV (25 - 267) \\
\hline & & & 28078 & BHV (18 - end $)$ \\
\hline & & & 55963 & BHV $(25-320)$ dimer $+\beta$-Trypsin \\
\hline \multirow{12}{*}{ BVB } & \multirow{7}{*}{5} & \multirow{7}{*}{2.5} & 44070 & BVB (18 - end) \\
\hline & & & 45863 & BVB \\
\hline & & & 67408 & BVB $(18$ - end $)+\beta$-Trypsin \\
\hline & & & 69194 & BVB $+\beta$-Trypsin \\
\hline & & & 89972 & BVB + BVB (18 - end $)$ \\
\hline & & & 91758 & BVB dimer \\
\hline & & & 115090 & BVB dimer $+\beta$-Trypsin \\
\hline & \multirow{5}{*}{5} & \multirow{5}{*}{10} & 23299 & $\beta$-Trypsin \\
\hline & & & 27351 & BVB (25 - 267) \\
\hline & & & 28078 & BVB (18 - 267) \\
\hline & & & 55957 & BVB $(25-320)+\beta$-Trypsin \\
\hline & & & 56684 & BVB $(18-320)+\beta$-Trypsin \\
\hline \multirow{7}{*}{$\mathrm{HBH}$} & \multirow{4}{*}{5} & \multirow{4}{*}{2.5} & 28323 & HBH (18-269) \\
\hline & & & 44488 & $\mathrm{HBH}(18$ - end $)$ \\
\hline & & & 67812 & HBH (18 - end) $+\beta$-Trypsin \\
\hline & & & 112336 & HBH (18 - end) dimer $+\beta$-Trypsin \\
\hline & \multirow{3}{*}{5} & \multirow{3}{*}{10} & 23293 & Trypsin \\
\hline & & & 27596 & HBH (25 - 269) \\
\hline & & & 28322 & HBH (18 - 269) \\
\hline \multirow{5}{*}{ VHV } & \multirow{5}{*}{5} & \multirow{5}{*}{2.5} & 28326 & VHV (18 - 269) \\
\hline & & & 43556 & VHV (18 - end) \\
\hline & & & 45350 & VHV \\
\hline & & & 87182 & VHV (18 - end) dimer \\
\hline & & & 88968 & VHV + VHV (18 - end $)$ \\
\hline
\end{tabular}


Table 4.3. Native ESI-MS masses observed in solutions of combinations of 6-trypsin and miniprotein chains. These measurements were performed by mixing the proteins prior to rebuffering them into aqueous AmAc. The mass spectra are presented in Figure 4.11 (see in the Experimental Section)

\begin{tabular}{|c|c|c|c|c|}
\hline $\begin{array}{l}\text { Miniprotein } \\
\text { chain }\end{array}$ & $\begin{array}{c}\text { Miniprotein } \\
\text { chain conc. } \\
(\mu \mathrm{M})\end{array}$ & $\begin{array}{l}\text { Trypsin conc. } \\
\qquad(\mu M)\end{array}$ & $\begin{array}{c}\text { Masses } \\
\text { obtained (Da) }\end{array}$ & Mass assignment \\
\hline \multirow{7}{*}{ BHV } & \multirow{7}{*}{5} & \multirow{7}{*}{5} & 23298 & $\beta$-Trypsin \\
\hline & & & 27357 & BHV $(25-267)$ \\
\hline & & & 28083 & BHV (18 - 267) \\
\hline & & & 55967 & BHV $(25$ - 320) dimer + $\beta$-Trypsin \\
\hline & & & 56694 & BHV $(18-320)$ dimer $+\beta$-Trypsin \\
\hline & & & 66638 & BHV (25 - end) $+\beta$-Trypsin \\
\hline & & & 67367 & BHV $(18$ - end $)+\beta$-Trypsin \\
\hline \multirow{5}{*}{ BVB } & \multirow{5}{*}{5} & \multirow{5}{*}{5} & 44079 & BVB (18 - end) \\
\hline & & & 45876 & BVB \\
\hline & & & 67436 & BVB $(18$ - end $)+\beta$-Trypsin \\
\hline & & & 69214 & BVB + $\beta$-Trypsin \\
\hline & & & 111611 & BVB (18 - end) dimer + $\beta$-Trypsin \\
\hline \multirow{4}{*}{$\mathrm{HBH}$} & \multirow{4}{*}{5} & \multirow{4}{*}{5} & 23296 & $\beta$-Trypsin \\
\hline & & & 26429 & $\mathrm{HBH}(165$ - 417) \\
\hline & & & 27598 & $\mathrm{HBH}(25-269)$ \\
\hline & & & 32336 & $\mathrm{HBH}(25-318)$ \\
\hline \multirow{2}{*}{ VHV } & \multirow{2}{*}{5} & \multirow{2}{*}{5} & 23330 & $\beta$-Trypsin \\
\hline & & & 27598 & VHV $(25-269)$ \\
\hline
\end{tabular}

\subsubsection{MST analysis of protein binding to miniprotein chains}

Taking advantage of the TFP fused with the miniprotein chains, we attempted to study the binding of the protein constructs with their target proteins using MST directly in buffer. In this technique, the fluorescence intensity is measured at a localized spot in solution, which is then heated using an infrared laser. Depending on the fluorescent molecule it will either diffuse away from or towards the heated spot. The rate of this diffusion depends on the hydration shell around the molecule, which in turn can change if something binds to it. For these measurements, we maintained the TFP-fused miniprotein chains at a constant concentration of $100 \mathrm{nM}$ and titrated it against a range of $\beta$-trypsin concentrations ( $0.2-7500 \mathrm{nM})$. The BHV, BVB and $\mathbf{H B H}$ samples showed a thermophoretic response in the presence of $\beta$-trypsin while VHV did not (Figure 4.4). The plots were fitted with the quadratic velocity equation (eq 4.3), ${ }^{36}$

$$
y=L+A m p \frac{F+x+K_{d}-\sqrt{\left(F+x+K_{d}\right)^{2}-4 F x}}{2 F} \quad \text { eq } 4.3
$$


where $L$ is the lower asymptotic value, Amp is the amplitude of the plot, $F$ is the concentration of the fluorescent molecule (the TFP-fused miniprotein chains), $x$ is the $\beta$-trypsin concentration and $K_{d}$ is the dissociation constant.

From Figure 4.4 , it can be seen that $K_{d}$ value was surprisingly similar (105 nM) for all three constructs containing $\mathbf{B}$, but the Amp value was slightly higher for BVB compared to BHV and $\mathbf{H B H}$. However from these plots, no distinct difference could be identified between the miniprotein chains consisting of one or two B knottins. Using MST, we were also unable to detect any thermophoretic response when the miniprotein chains were titrated with VEGF or HIV-1 Nef (data not shown).

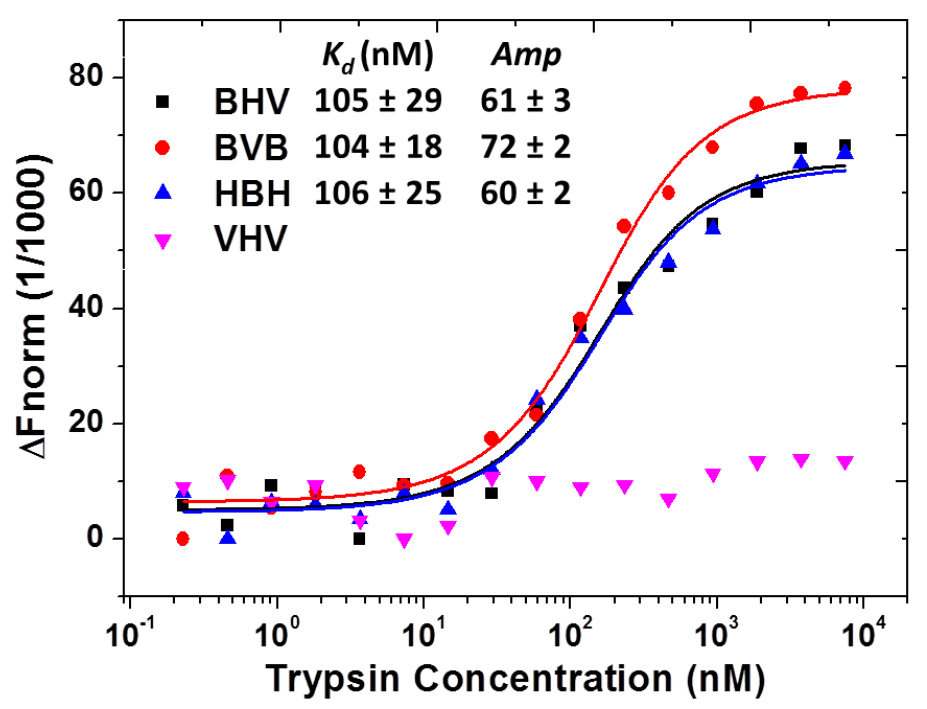

Figure 4.4. MST data for the four miniprotein chains titrated against a range of 8 -trypsin concentrations. The solid lines represent fittings using the quadratic velocity equation (eq 4.4).

\subsubsection{SPR analysis of protein binding to miniprotein chains}

To simultaneously follow the binding of the different proteins with the miniprotein chains, we performed SPR imaging (SPR-i) measurements. Multiplexed label-free measurements were possible using a hemispherical glass sensor with a gold layer on flat surface coated with a dextran polymer containing activated ester groups. Using a Continuous Flow Microspotter, which incorporates a PDMS print head with 48 flow channels, various proteins can be spotted onto the sensor surface in arrays. The 
amount of proteins immobilized onto the surface was determined by the initial SPR response obtained and is termed Response of Local Ligand (RLL). In the first set of experiments, we spotted $\beta$-trypsin, VEGF16 5 and HIV-1 Nef proteins at different loading densities. A range of TFP-fused miniprotein chain concentrations ( $1 \mathrm{nM}-2 \mu \mathrm{M})$ were then flowed over the spotted sensor while monitoring the SPR image. The best responses obtained from each sensor were taken and the values were normalized by dividing by the RLL values of the corresponding spots. From Figure 4.5, it can be seen that for example BHV binds to each of the three spotted proteins. Figure 4.5a shows the responses from the binding of increasing concentrations of the miniprotein chains on spots with $\beta$-trypsin. BHV, BVB and $\mathbf{H B H}$ clearly bind to the spots while VHV does not. In addition, BVB did not bind to HIV-1 Nef while HBH did not bind significantly to VEGF. These results, verify the specific binding of $\mathbf{B}$ knottin to trypsin. The highest response was seen with $\mathbf{B H V}$, possibly due to the position of $\mathbf{B}$ in the chain, allowing for better packing of the proteins. Even though BVB caused a lower response, binding started at a lower concentration compared to $\mathbf{B H V}$ and $\mathbf{H B H}$, indicating a higher affinity. This was verified by fitting the binding plots with the Langmuir adsorption equation as shown in Figure 4.5d. The lower $K_{d}$ value obtained for BVB indicated the possibility of bivalent binding occurring at the surface. Surprisingly and contrary to our previous results in solutions, $\mathbf{H B H}$ showed the weakest response and the poorest affinity. This could also possibly be due to the position of $\mathbf{B}$ in the chain, causing some sort of unexpected steric hindrance with immobilized $\beta$-trypsin while binding. From Figure $4.5 \mathrm{~b}$, it can be seen that both BHV and BVB were binding to spots with VEGF165 to a similar extent. Surprisingly, a very weak, nearly negligible response was observed for the binding of VHV to VEGF spots, even though binding with a higher affinity would be expected. This indicates possible errors in the folding of this miniprotein chain or some sort of interaction between the miniproteins within the chain causing steric hindrance and preventing it from binding VEGF165. Even though $\mathbf{H B H}$ is considered to be a negative control in this case, some non-specific adhesion is seen at higher concentrations. A blocking agent such as bovine serum albumin (BSA) might be required to suppress such non-specific adhesion, as is normally used in ELISA experiments dealing with growth factors. In Figure $4.5 \mathrm{C}$, it can be seen that BHV and HBH bind HIV-1 Nef to a significant extent while the negative control BVB does not cause any response. Once again, almost no response is observed from VHV, supporting our suspicion that this miniprotein chain is not functional. It is important to note that this is the only miniprotein chain that is made entirely out of Min-23 constructs. Binding affinities of the miniprotein chains with VEGF165 and HIV-1 Nef were not determined 
since the responses still lay only in the linear regime, for which fitting with the Langmuir adsorption equation is not reliably applicable.

Next, we tested the multi-specific binding properties of the miniprotein chains by spotting them on the surface and flowing over VEGF165 and HIV-1 Nef while recording the SPR-i responses. In Figure 4.5e, VEGF165 $(1 \mu \mathrm{M})$ was first flowed over the sensor, allowed to bind, rinsed then HIV-1 Nef $(1 \mu \mathrm{M})$ was passed. In this case, the best VEGF165 binding was observed to occur with BHV and VHV. It is possible that in the immobilized form, the $\mathbf{V}$ miniproteins of the VHV chain were conjugated in a more accessible manner for binding. This is an indication that the folding might not be incorrect but that the miniproteins within the chain cluster together and restrict access for binding. Significant non-specific binding was seen to occur with $\mathbf{H B H}$ probably since the VEGF165 concentration used was relatively high $(1 \mu M)$. Surprisingly, very low binding seemed to occur with BVB, possibly even only non-specifically, indicating that the position of $\mathbf{V}$ in this immobilized miniprotein chain was probably unfavourable for binding. On the other hand, HIV-1 Nef binding complied with our earlier results, binding most significantly to $\mathbf{B H V}$ and $\mathbf{H B H}$, much less with VHV and slightly non-specifically with BVB. However the response from $\mathbf{B H V}$ and $\mathbf{H B H}$ were the same, providing no indications of multiple binding. In a similar experiment, we flowed HIV-1 Nef $(1 \mu M)$ followed by a solution containing both VEGF165 $(1 \mu \mathrm{M})$ and HIV-1 Nef $(1 \mu \mathrm{M})$ without any washing steps in between (Figure 4.5f). The responses on the BHV spot seemed to indicate simultaneous multi-specific binding of both proteins. On BVB, HIV-1 Nef was quite weak while VEGF165 bound significantly, as expected. However on HBH, HIV-1 Nef binding was less than on BHV and non-specific VEGF165 binding was also considerable. On VHV, the worst binding of both proteins were seen, similar to what was witnessed in Figures 4.5a-c. $\beta$-trypsin was not included in these measurements, since it seemed to degrade even the gel on top of the gold sensor as shown in Figure $4.5 f$ (inset). The response dropped on the blank spot and the immobilized miniprotein chains also got removed from their spots due to trypsin. These results together indicate the multiple and multi-specific binding events occurred between the miniprotein chains and their target proteins. However several issues dealing with folding, miniprotein orientations, non-specific adhesion and proteolytic degradation of the miniproteins need to be carefully investigated and optimized before applications of these type of miniprotein chains can be undertaken. 
a) $\beta$-trypsin

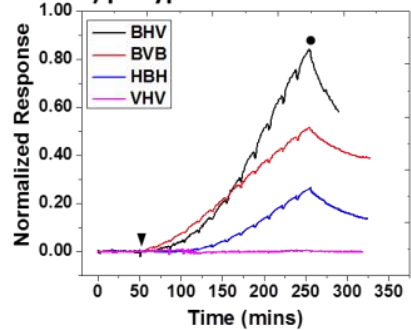

d) $\beta$-trypsin binding curves

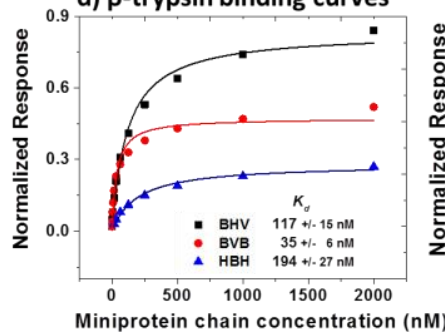

b) VEGF165

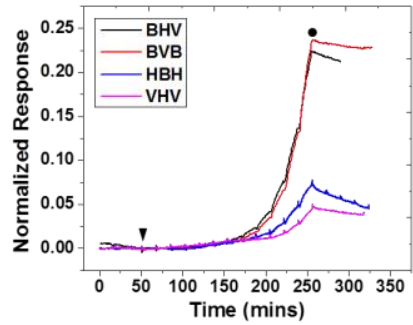

e) VEGF165, HIV-1 nef

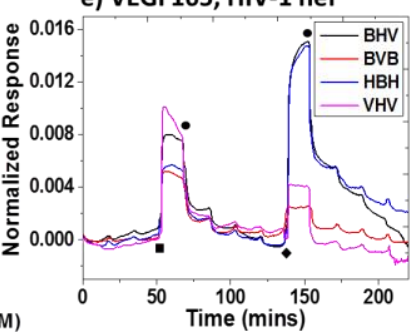

c) HIV-1 nef
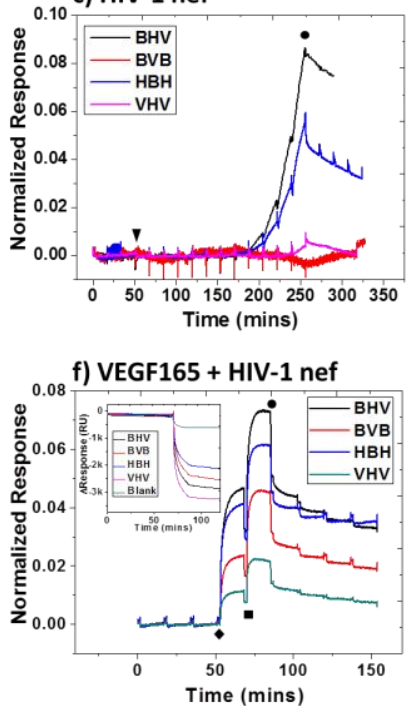

Figure 4.5. Normalized SPR-i responses obtained by flowing a range of concentrations $(1,2,4,8,16$, $31,62,125,250,500,1000,2000 \mathrm{nM})$ of BHV, BVB, HBH and VHV on spots of a) 6 -trypsin (RLL = 255 with BHV, 653 with BVB, 1080 with HBH and 510 with VHV), b) VEGF165 (RLL = 1820 with BHV, 2322 with BVB, 1774 with HBH and 2273 with VHV), c) HIV-1 Nef (RLL = 2143 with BHV, 982 with BVB, 2761 with $\mathrm{HBH}$ and 2572 with VHV). The black inverted triangle represents the start of the titration after baseline with system buffer and the black circle represents rinsing with system buffer. d) Maximum responses at each concentration of the miniprotein chains flowed over spots of B-trypsin plotted against the corresponding concentrations. The solid lines represent fitting with Langmuir adsorption equation. e) Normalized SPR-i responses obtained by flowing $1 \mu \mathrm{M}$ of VEGF165 (black square) and $1 \mu \mathrm{M} \mathrm{HIV-1}$ Nef (black diamond) over spots with BHV $(R L L=5186), B V B(R L L=5024)$, HBH (RLL = 5047) and VHV (RLL = 6197) with a buffer rinsing step after each protein (black circle). $f)$ Normalized SPR-i responses obtained by flowing over spots of BHV $(R L L=6013)$, BVB $(R L L=6091)$, HBH $(R L L=6256)$ and VHV $(R L L=8522)$ with $1 \mu \mathrm{M} \mathrm{HIV-1} \mathrm{Nef} \mathrm{(black} \mathrm{diamond)} \mathrm{immediately} \mathrm{followed}$ by $1 \mu M$ HIV-1 Nef + $1 \mu$ M VEGF165 (black square) after which buffer rinsing was done (black circle). The inset represents the flowing of 6 -trypsin over spots of immobilized miniprotein chains and also a blank spot. All normalized values were obtained by taking actual SPR-i responses, in Resonance Units, and dividing them by the corresponding RLL values.

\subsection{Conclusions}

We attempted to construct robust multivalent and multi-specific proteins by linking together the cystine-stabilized miniproteins, knottins and Min-23 derivatives, in the form of a chain. An efficient strategy of genetically constructing these recombinant proteins was successfully employed. Protein expression in E.coli and purification were 92 
facilitated by fusing these chains with a hexa-His-tagged TFP. SDS-PAGE and Native ESIMS were used to characterize the purified proteins and confirm their complete synthesis. A trypsin enzymatic activity assay was used to verify that the $\beta$-trypsin inhibitory knottins in the miniprotein chains were functional, further indicating that they had undergone proper folding. An indication of multiple binding was also seen with this assay since the two binding site construct exhibited stronger inhibition of $\beta$ trypsin compared to the one binding site constructs. Native ESI-MS also revealed that the miniprotein chains formed complexes with $\beta$-trypsin and were relatively resistant to its proteolytic activity. MST further confirmed binding of the miniprotein chains with $\beta$ trypsin at $\mathrm{nM}$ affinities. With SPRi studies, multiple and multi-specific binding of the target proteins with the miniprotein chains was observed, however certain problems with the current system were also revealed. Further studies are required to optimize the linker properties, eliminate non-specific binding, determine folding parameters, and reduce proteolysis even more. The system shows considerable promise for developing highly stable scaffold proteins that can simultaneously bind multiple different targets for therapeutic and industrial applications. Alternatively, such miniprotein chains could possibly be constructed by using $\mathrm{CB}[8]$-binding peptide motifs similar to those described in Chapter 3. This would add another dimension to the properties of these chains since the constituent miniproteins would be able to dynamically shift positions within the chain and possibly even respond to external stimuli.

\subsection{Acknowledgements}

We would like to thank Prof. Luc Brunsveld for providing access to his MST device for initial measurements and training. The facilities of the BioNanoLab of the MESA Institute for Nanotechnology are highly appreciated.

\subsection{Experimental Section}

Materials. Bovine $\beta$-trypsin and BAEE were obtained from Sigma Aldrich. Recombinant VEGF165 was purchased from Peprotech. Recombinant HIV-1 Nef was purchased from Jena Bioscience in a wild-type, His-tagged, myristoylated form.

Equipment. Polymerase Chain Reaction (PCR) was performed using a Peqlab Primus 25 advanced thermocycler. UV-Vis measurements to determine DNA and protein concentrations were performed using a Thermo Scientific Nanodrop 1000. UV-Vis measurements for trypsin inhibitor assays were performed using a Perkin Elmer Victor $\mathrm{X}_{3}$ multiwell plate reader. Mass spectrometry measurements were performed in positive ion mode using an LCT electrospray time-of-flight (Waters, UK). Needles were 
made from borosilicate glass capillaries (Kwik-Fil, World Precision Instruments, Sarasota, FL) on a P97 puller (Sutter Instruments, Novato, USA), coated with a thin gold layer by using an Edwards Scancoat six pirani 501 sputter coater (Edwards Laboratories, Milpitas, USA). Miscroscale thermophoresis measurements were performed using a Nanotemper Monolith NT.115 device. Continuous flow microspotting was performed with a 48 channel PDMS CFM device from Wasatch microfluidics LLC. Spotting was performed at $150 \mu \mathrm{L} / \mathrm{min}$ back and forth flow for an hour. For SPR-i measurements the IBIS MX96 was used (IBIS technologies B.V., Enschede, the Netherlands).

Molecular cloning of miniprotein chains. pET15b TFP-Ecoil vector was obtained from W. F. Rurup. ${ }^{37}$ ssDNA sequences corresponding to $\mathbf{B}, \mathbf{V}$ and $\mathbf{H}$ miniproteins and primers to extend the $\mathrm{N}$ and $\mathrm{C}$ termini with the appropriate linker sequences and restriction sites, were ordered from Eurofins MWG Operon, Germany. Amplification of the extended genetic construct was performed by PCR using pfu polymerase. The final genetic construct had 5' BsrGI and 3' Nhel restriction sites, which were used to insert it between the TFP and Ecoil in the plasmid through restriction digestion enzymes and T4 DNA ligase (New England Biolabs inc.). The resulting pET15b TFP-BHV, pET15b TFP-BVB, PET15b TFP-HBH, pET15b TFP-VHV plasmids were transformed into NovaBlue ultracompetent cells (Novagen) and grown overnight on LB agar plates containing 100 $\mathrm{mg} / \mathrm{L}$ ampicillin. Plasmids were extracted from individual colonies using Qiagen spin miniprep kit and the sequenced by Eurofins MWG Operon, Germany using a standard T7 terminal reverse primer. The four different plasmids were transformed into the expression host, Rosetta-Gami 2(DE3)pLysS competent cells (Novagen) and grown overnight on LB agar plates containing $34 \mathrm{mg} / \mathrm{L}$ chloramphenicol and 10omg/L ampicillin. Individual colonies were grown in LB media containing the mentioned antibiotics. For long term storage, $15 \%$-glycerol bacterial stocks were made and placed at $-80^{\circ} \mathrm{C}$. 


\section{DNA sequence of BHV:}

5'-

atgggcagcagcCATCATCATCATCATCACagcagcggcCTGGTGCCGCGCGGGAGCCatATGGTGA GCAAGGGCGAGGAGACCACAATGGGCGTAATCAAGCCCGACATGAAGATCAAGCTGAAGATG GAGGGCAACGTGAATGGCCACGCCTTCGTGATCGAGGGCGAGGGCGAGGGCAAGCCCTACGA CGGCACCAACACCATCAACCTGGAGGTGAAGGAGGGAGCCCCCCTGCCCTTCTCCTACGACATT CTGACCACCGCGTTCGCCTACGGCAACAGGGCCTTCACCAAGTACCCCGACGACATCCCCAACT ACTTCAAGCAGTCCTTCCCCGAGGGCTACTCTTGGGAGCGCACCATGACCTTCGAGGACAAGG GCATCGTGAAGGTGAAGTCCGACATCTCCATGGAGGAGGACTCCTTCATCTACGAGATACACC TCAAGGGCGAGAACTTCCCCCCCAACGGCCCCGTGATGCAGAAGAAGACCACCGGCTGGGACG CCTCCACCGAGAGGATGTACGTGCGCGACGGCGTGCTGAAGGGCGACGTCAAGCACAAGCTGC TGCTGGAGGGCGGCGGCCACCACCGCGTTGACTTCAAGACCATCTACAGGGCCAAGAAGGCGG TGAAGCTGCCCGACTATCACTTTGTGGACCACCGCATCGAGATCCTGAACCACGACAAGGACT ACAACAAGGTGACCGTTTACGAGAGCGCCGTGGCCCGCAACTCCACCGACGGCATGGACGAGC TGTACAatCCAGCACCAGCGTCAGGTTCAGGTTCAGGTCGCGTGTGCCCGCGCATTCTGATGGA ATGCAAAAAAGATAGCGATTGCCTGGCGGAATGCGTGTGCCTGGAACATGGCTATTGCGGCGG AAGTGGAAGTGGAAGTGCCCCTGCTCCTGAGCTCCCAGCACCAGCGTCAGGTTCAGGTTCAGG TCTGATGCGCTGCAAACAGGATAGCGATTGCCTGGCGGGCAGCGTGTGCACCCAGGTGCTGAG CTTTGTGCCGTGGAAATTTTGCGGCGGAAGTGGAAGTGGAAGTGCCCCTGCTCCTCTTAAGCCA GCACCAGCGTCAGGTTCAGGTTCAGGTCTGATGCGCTGCCGCAGCGATGCGGATTGCATTGAA GGCCGCGTGTGCAGCTATTTTAGCCAGGGCTTTTGCGGCGGAAGTGGAAGTGGAAGTGCCCCT GCTCCTTCGCTAGCTGAAATTGCTGCTCTTGAAAAAGAAATTGCTGCTCTTGAAAAAGAAATTG CTGCTCTTGAAAAATAA

$-3$

Protein Sequence of BHV:

MGSSHHHHHHSSGLVPRGSHMVSKGEETTMGVIKPDMKIKLKMEGNVNGHAFVIEGEGEGKP YDGTNTINLEVKEGAPLPFSYDILTTAFAYGNRAFTKYPDDIPNYFKQSFPEGYSWERTMTFEDK GIVKVKSDISMEEDSFIYEIHLKGENFPPNGPVMQKKTTGWDASTERMYVRDGVLKGDVKHKLL LEGGGHHRVDFKTIYRAKKAVKLPDYHFVDHRIEILNHDKDYNKVTVYESAVARNSTDGMDELY NPAPASGSGSGRVCPRILMECKKDSDCLAECVCLEHGYCGGSGSGSAPAPELPAPASGSGSGLM RCKQDSDCLAGSVCTQVLSFVPWKFCGGSGSGSAPAPLKPAPASGSGSGLMRCRSDADCIEGRV CSYFSQGFCGGSGSGSAPAPSLAEIAALEKEIAALEKEIAALEK*

Blue = Hexa-His Tag, Gray $=$ Thrombin cleavage site, Dark Green $=$ TFP, Orange $=$ $(\mathrm{PA})_{2} /(\mathrm{AP})_{2}$, Brown $=(\mathrm{SG})_{3} /(\mathrm{GS})_{3}$, Light green $=\mathbf{B}$, Purple $=\mathbf{H}$, Red $=\mathbf{V}$, Yellow $=$ E-coil leucine zipper 
Primers used:

Forward:

BsrGI-(PA) 2 -(SG) 3 - 5'-agtgtaTGTACAatCCAGCACCAGCGTCAGGTTCAGGTTCAGGT-3'

Sacl-(PA) 2 -(SG) 3 - 5'-agtgtaGAGCTCCCAGCACCAGCGTCAGGTTCAGGTTCAGGT-3'

AfIII-(PA) 2 -(SG) 3 -5'-agtgtaCTTAAGCCAGCACCAGCGTCAGGTTCAGGTTCAGGT-3'

Ndel-(PA) 2 -(SG) 3 -5'-agtgtaCATATGCCAGCACCAGCGTCAGGTTCAGGTTCAGGT-3'

Reverse:

Sacl-(AP) $)_{2}-(G S)_{3}-5$ '-atgtgaGAGCTCAGGAGCAGGGGCACTTCCACTTCCACTTCC-3'

AfIII -(AP) $)_{2}-(\mathrm{CS})_{3}-5$ '-atgtgaCTTAAGAGGAGCAGGGGCACTTCCACTTCCACTTCC-3'

Nhel-(AP) $)_{2}-(G S)_{3}-5$ '-atgtgaGCTAGCgaAGGAGCAGGGGCACTTCCACTTCCACTTCC-3'

BlpI-(AP) $)_{2}-(G S)_{3}-5$ '-atgtgataGCTCAGCAGGAGCAGGGGCACTTCCACTTCCACTTCC-3'

Protein expression and purification. $5 \mathrm{ml}$ bacterial starter cultures were grown overnight from glycerol stocks at $37^{\circ} \mathrm{C}$ with shaking in LB media containing appropriate antibiotics. This was then transferred into $1 \mathrm{~L}$ of the same media and the cultures were grown till they attained O.D.6oonm values between 0.4 and 0.8 . Protein expression was then induced using isopropyl- $\beta$-D-1-thiogalactopyranoside (IPTG) at a final concentration of $0.1 \mathrm{mM}$. These cultures were grown overnight at $180^{\circ} \mathrm{C}$ with shaking. The cultures were then spun down at $6,000 \mathrm{rcf}$ for $10 \mathrm{mins}$ at $40^{\circ} \mathrm{C}$ and supernatants were discarded. Bacterial pellets were resuspended in $10 \mathrm{~mL}$ BugBuster protein extraction reagent (Novagen) with $10 \mu \mathrm{L}$ Benzonase (30 $\mathrm{U} / \mu \mathrm{L}$, Novagen) and gently shaken for 20 mins at $25^{\circ} \mathrm{C}$. TFP-fused knottin constructs were then purified using HisSelect $\odot$ Nickel affinity columns (Sigma Aldrich) into an elution buffer $(\mathrm{pH} \mathrm{8)}$ of $50 \mathrm{mM}$ $\mathrm{NaH}_{2} \mathrm{PO}_{4} 300 \mathrm{mM} \mathrm{NaCl} 250 \mathrm{mM}$ imidazole. The purified TFP-fused knottins were then rebuffered into $\mathrm{pH} 7.4$ phosphate buffered saline (PBS, Sigma Aldrich) using $30 \mathrm{kDa}$ cutoff centrifugal filter units (Amicon Ultra). Concentrations of these TFP-fused miniprotein chains were determined from the absorbance value at $\lambda=467 \mathrm{~nm}$ and an extinction coefficient of $64,000 \mathrm{M}^{-1} \mathrm{~cm}^{-1}$.

Trypsin inhibitor assay. $\beta$-trypsin cleaves peptides and proteins mainly at the carboxyl side of the amino acids lysine or arginine. During this experiment BAEE was used as a substrate for trypsin digestion. This produces an absorbance shift from $\lambda=230 \mathrm{~nm}$ to $253 \mathrm{~nm}$. The rate of reaction was determined for different concentrations of BAEE in 
the presence and absence of the knottins by following the increase in absorbance using a $260 \mathrm{~nm}$ filter. All measurements were performed in PBS.

Microscale Thermophoresis. Solutions containing $100 \mathrm{nM}$ TFP-fused miniprotein chain were titrated with serially diluted concentrations of $\beta$-trypsin ranging from $0.2 \mathrm{nM}$ to 7.5 $\mu \mathrm{M}$ in PBS. Thermophoretic analyses were carried out in standard treated capillaries from Nanotemper using an LED power of $40 \%$ and MST power of $100 \%$ for $30 \mathrm{~s}$. Thermophoresis was calculated as the ratio of the fluorescence intensity after and before local heating.

SPRi. Easy 2 Spot ${ }^{\circledR}$ SensEye ${ }^{\circledR}$ pre-activated G-type sensors (Ssens bv, Enschede, The Netherlands) were used as gold SPR sensor surfaces. The sensors have a $100 \mathrm{~nm}$ hydrogel-like layer with pre-activated ester groups, enabling high capacity coupling of proteins on the surface. Proteins were spotted on the sensor surfaces using a Continuous Flow Microspotter (CFM) spotter. The CFM spotter can spot up to 48 different ligands onto the sensor chip simultaneously under back and forth confined flow. For the spotting procedure, a $10 \mathrm{mM}$ sodium acetate buffer with $\mathrm{pH} 4.5$ containing $0.003 \%$ Tween-20 was used as the immobilization buffer in which the proteins solutions were made. Spotting was done for 30 mins using $150 \mu \mathrm{L}$ of protein solutions with concentrations serially diluted down from $5 \mu \mathrm{g} / \mathrm{mL}$ to $0.08 \mu \mathrm{g} / \mathrm{mL}$ after which the spots were briefly washed with the immobilization buffer for 2 mins. The spotted sensor was then transferred to the IBIS MX96 system for SPRi measurements and the RLL values of the signal spots were determined by subtracting the SPR-dips of the local reference spots. This system also uses back and forth flow for minimizing the amount of sample and reagents needed for a measurement. 1\% BSA followed by $100 \mathrm{mM}$ ethanolamine in immobilization buffer were flowed over the sensor for 8 mins each to deactivate the areas outside the spots and also unreacted ester groups within the spots. On the SPR image available, regions of interest (ROIs) were placed corresponding to the spotted regions from which measurements were recorded. Blank spots and unspotted areas were used as reference. For the SPR-i measurements, proteins were dissolved in the system buffer made up of PBS and $0.003 \%$ Tween-20, $\mathrm{pH}$ 7.4. The measurements in Figures $4.5 \mathrm{a}$-c followed the following sequence -3 rounds of system buffer, 12 rounds of increasing concentrations of miniprotein chains $(1,2,4,8$, 16, 31, 62, 125, 250, 500, 1000, $2000 \mathrm{nM}$ ), 2 rounds of system buffer. Each round followed the following sequence of solutions - 1 min system buffer (baseline), 15 mins solution pertaining to that round (association), $4-5$ s wash step with system buffer. For each round $150 \mu \mathrm{L}$ solution was used. Initial flow speed to bring the appropriate 
solution to the chip was $20 \mu \mathrm{L} / \mathrm{s}$ after which back and forth flow was maintained at 14 $\mu \mathrm{L} / \mathrm{s}$. All measurements were carried out at $25^{\circ} \mathrm{C}$.

Native ESI-MS. The TFP-fused miniprotein chains, $\beta$-trypsin, VEGF16 5 and HIV-1 Nef were buffer exchanged to $0.150 \mathrm{M}$ ammonium acetate $(\mathrm{pH} 7.5)$ at $4^{\circ} \mathrm{C}$, using seven sequential steps on a centrifugal filter with a cut-off of $10 \mathrm{kDa}$ (Sartorius). The complex was sprayed at a concentration of 1-10 $\mu \mathrm{M}$ from borosilicate glass capillaries. An LCT electrospray time-of-flight instrument (Waters, UK) adjusted for optimal performance in high mass detection was used. Instrument settings were as follows - needle voltage around $1200 \mathrm{~V}$, cone voltage around $100 \mathrm{~V}$, source pressure $6.5 \mathrm{mbar}$. All spectra were calibrated using a solution of $25 \mathrm{mg} / \mathrm{ml}$ Csl. Exact mass measurements of the individual miniprotein chains were acquired under denaturing conditions ( $5 \%$ formic acid). All spectra associated with the tables in the main text have been provided here. 


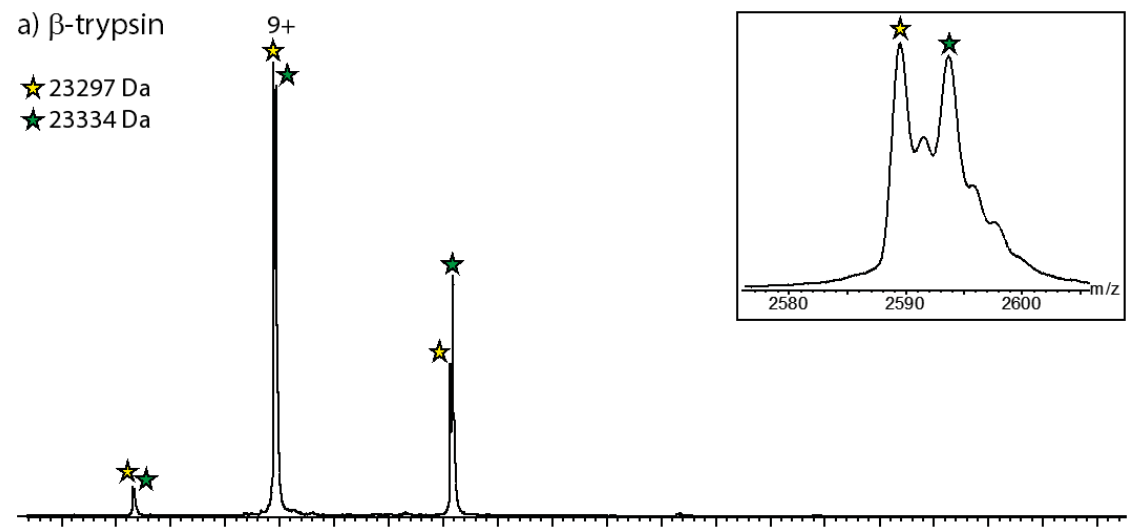

b) VEGF165

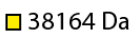

$\square 38280 \mathrm{Da}$

ㄱ38402 Da
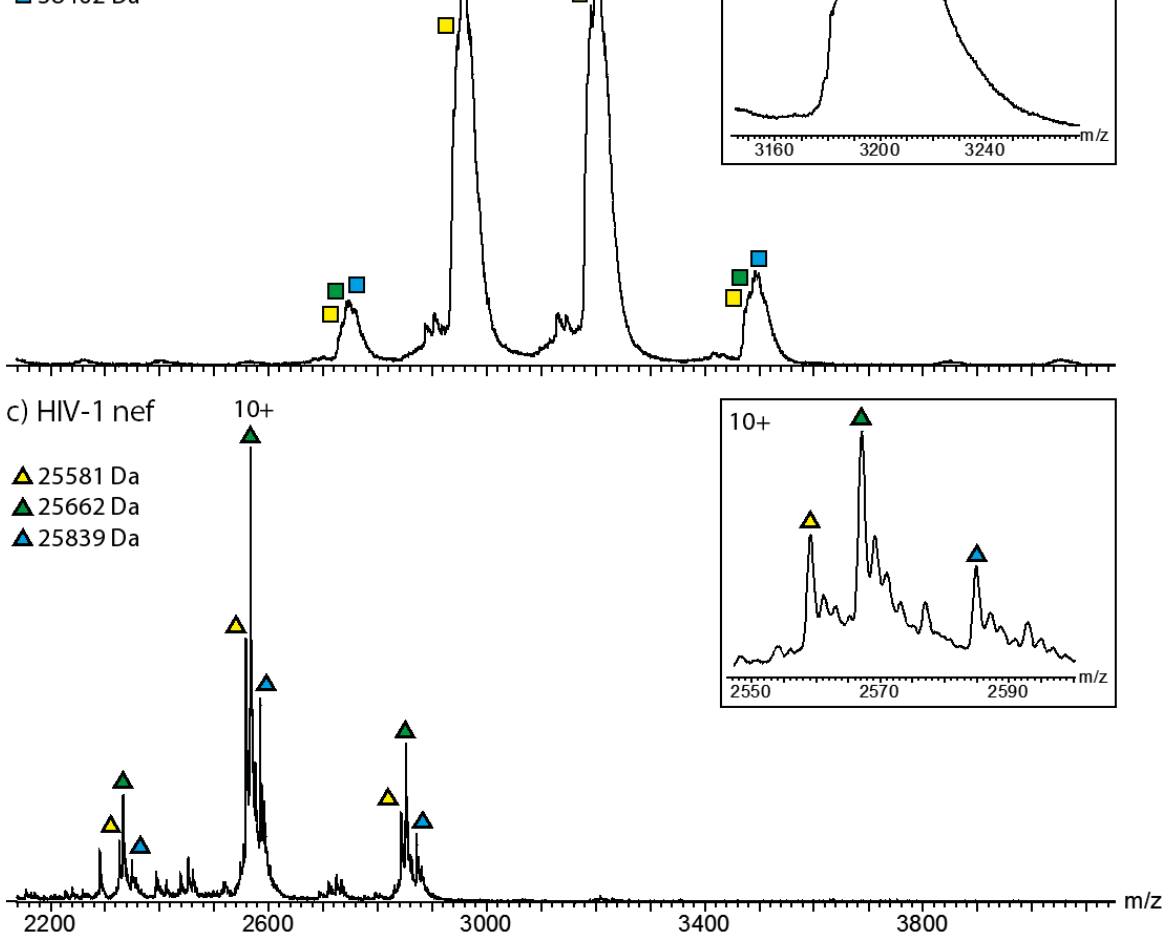

Figure 4.6. Native mass spectra of a) B-trypsin, b) VEGF165 and c) HIV-1 Nef. 

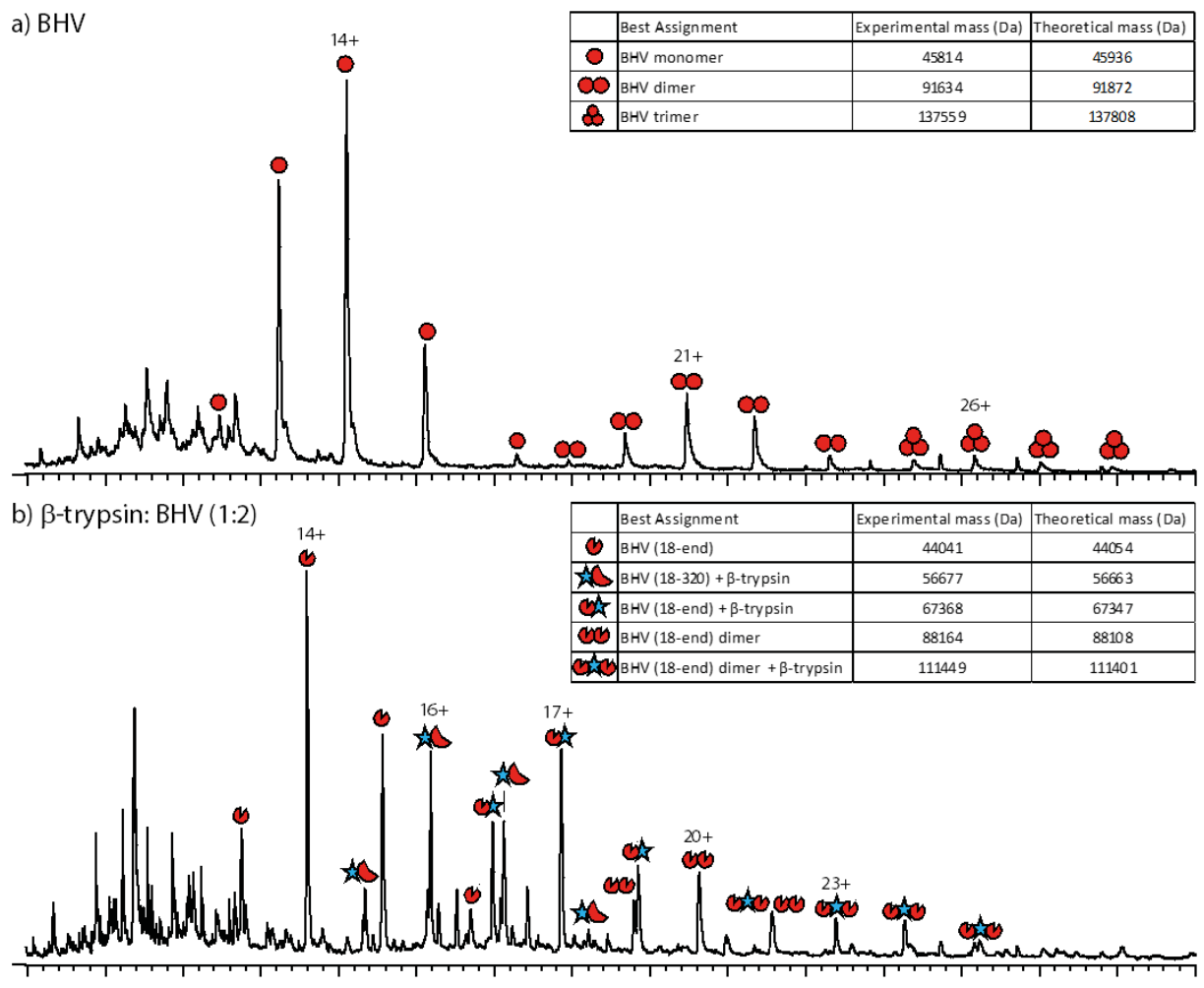

c) $\beta$-trypsin: $\mathrm{BHV}(2: 1)$

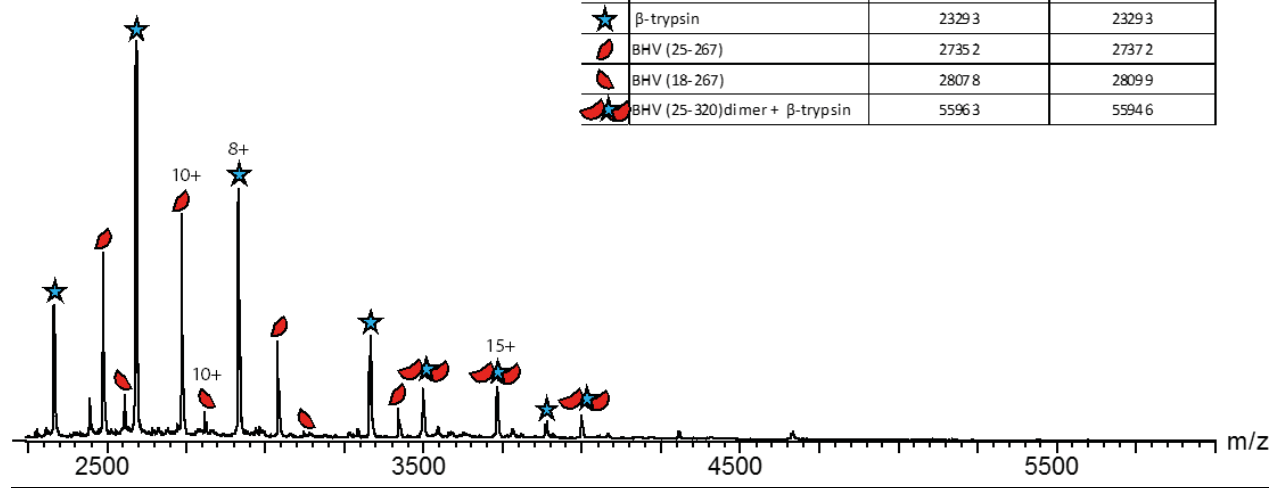

Figure 4.7. Native mass spectra of a) BHV, b) 8-trypsin:BHV (1:2) and c) 8-trypsin:BHV (2:1) 


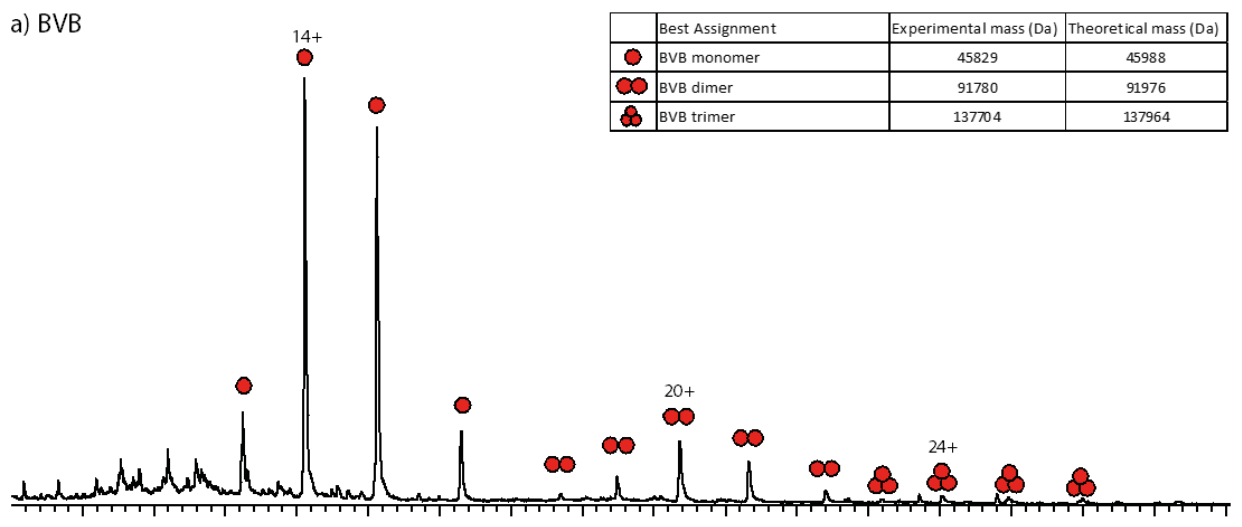

b) $\beta$-trypsin: BVB (1:2)

\begin{tabular}{|c|l|c|c|}
\hline & Best Assignment & Experimental mass (Da) & Theoretical mass (Da) \\
\hline 0 & BVB (18-end) & 44070 & 44106 \\
\hline 0 & BVB & 45863 & 45988 \\
\hline B & BVB (18-end)+ $\beta$-trypsin & 67408 & 67399 \\
\hline B & BVB + $\beta$-trypsin & 69194 & 69282 \\
\hline CO & BVB + BVB (18-end) & 89972 & 89933 \\
\hline BV & BVB dimer & 91758 & 91976 \\
\hline
\end{tabular}

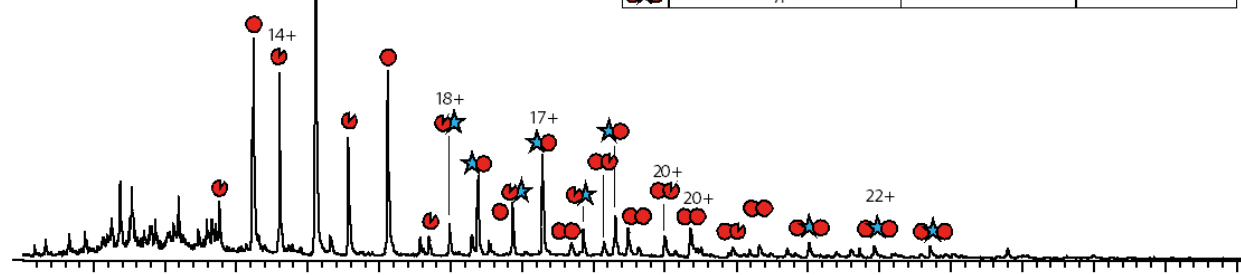

c) $\beta$-trypsin: BVB (2:1)

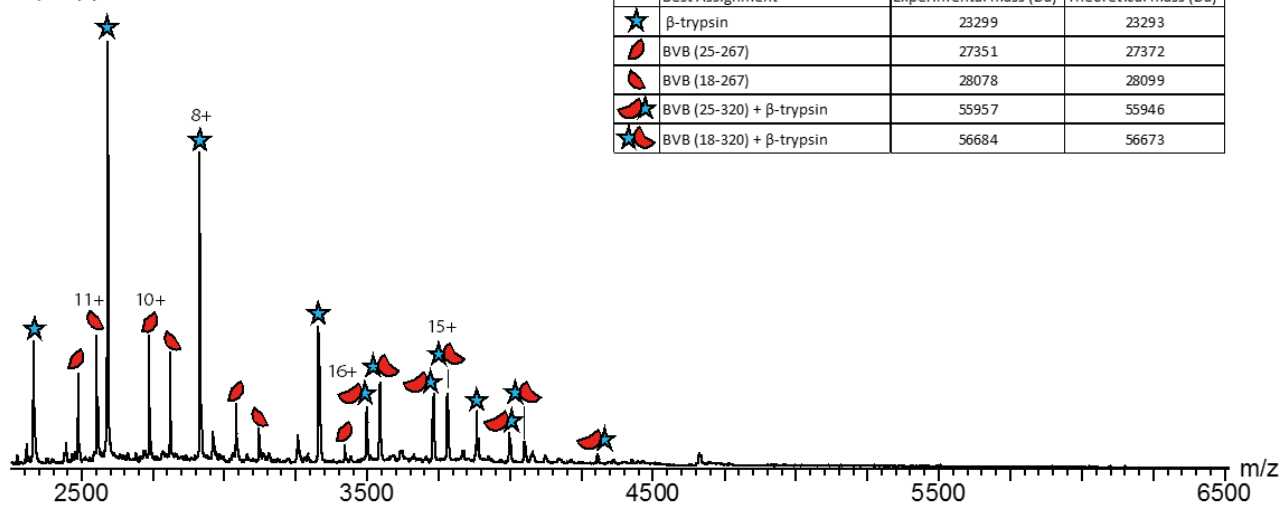

Figure 4.8. Native mass spectra of a) $B V B, b)$-trypsin:BVB (1:2) and c) 6 -trypsin:BVB (2:1) 
a) $\mathrm{HBH}$

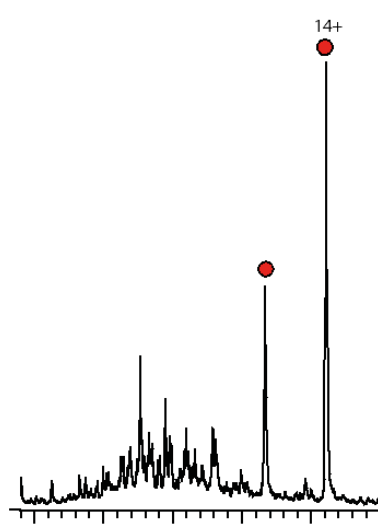

\begin{tabular}{|c|l|c|c|}
\hline & Best Assignment & Experimental mass (Da) & Theoretical mass (Da) \\
\hline $\mathrm{O}$ & HBH & 46236 & 46355 \\
\hline $\mathbf{C O}$ & HBH dimer & 92509 & 92709 \\
\hline $\mathbf{8}$ & HBH trimer & 138746 & 139064 \\
\hline
\end{tabular}

b) $\beta$-trypsin: $\mathrm{HBH}(1: 2)$

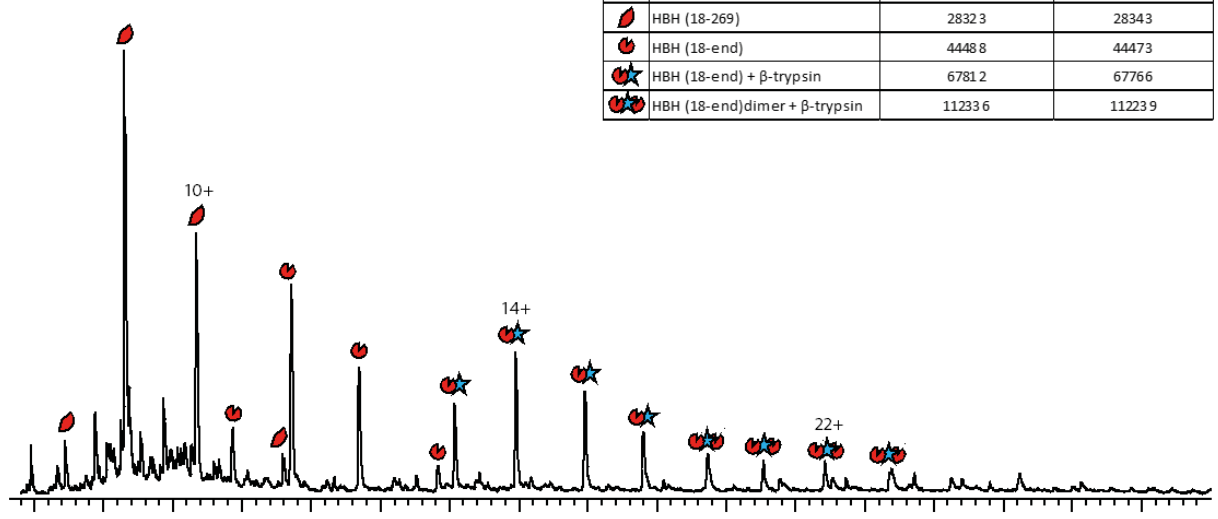

c) $\beta$-trypsin: $\mathrm{HBH}(2: 1)$

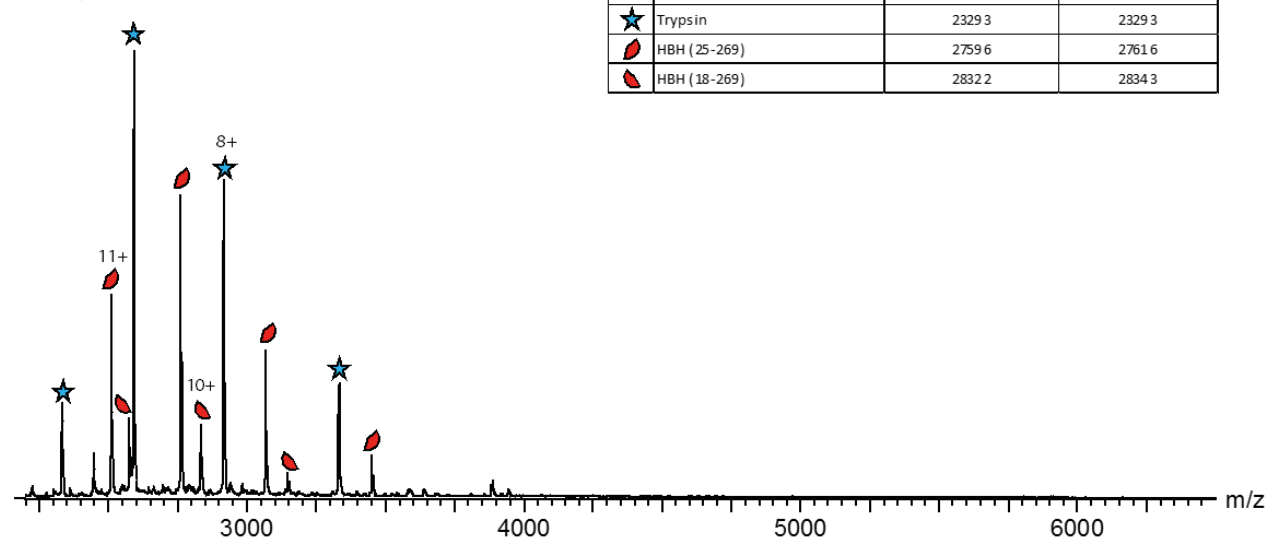

Figure 4.9. Native mass spectra of a) HBH, b) b-trypsin:HBH (1:2) and c) b-trypsin:HBH (2:1) 


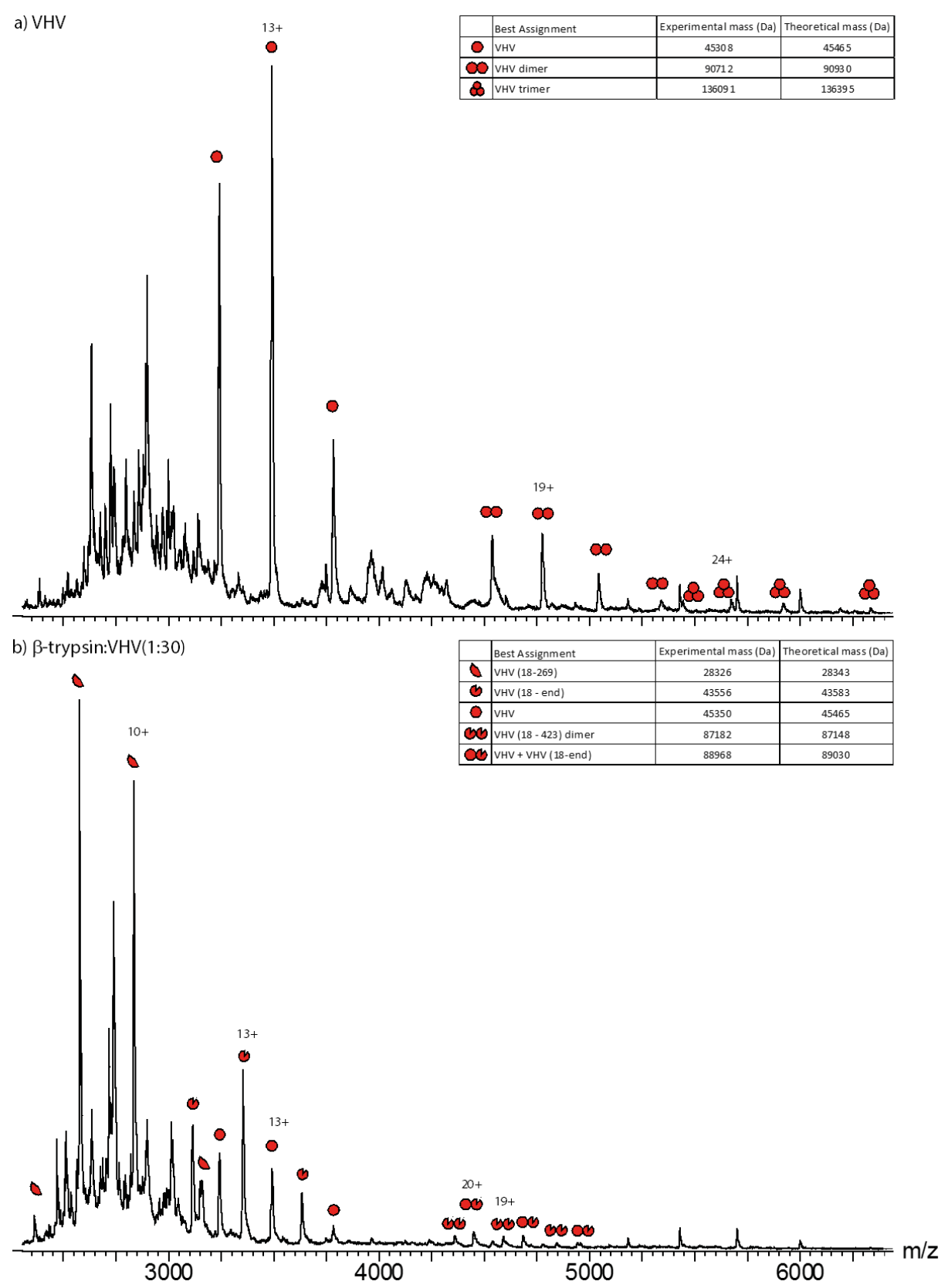

Figure 4.10. Native mass spectra of a) VHV and b) b-trypsin:VHV (1:2). 

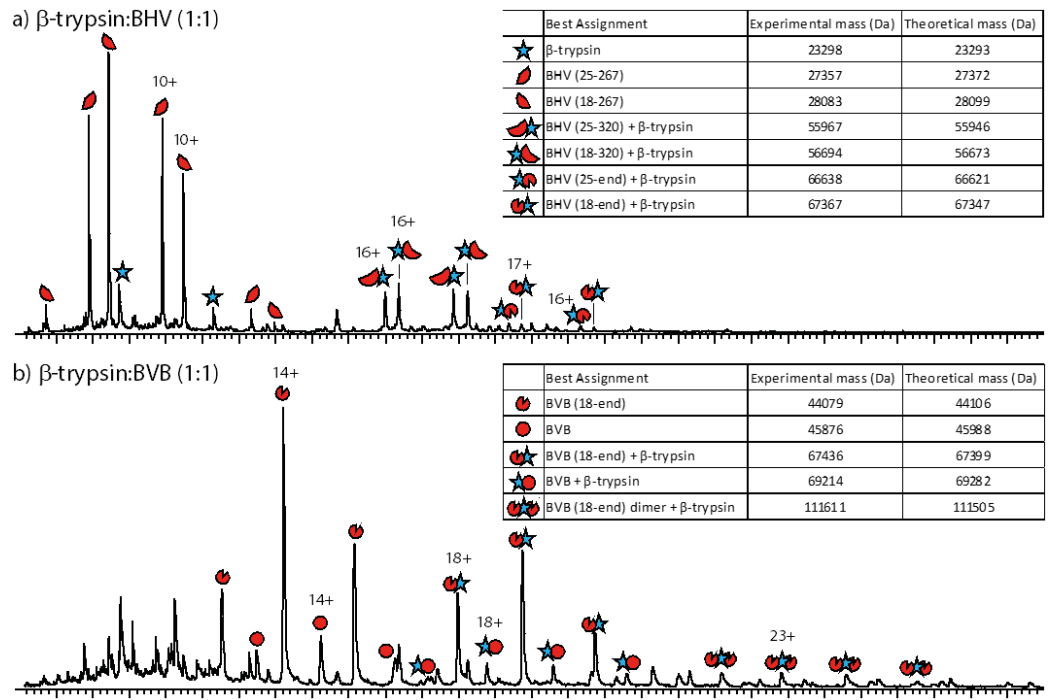

c) $\beta$-trypsin: $\mathrm{HBH}(1: 1)$

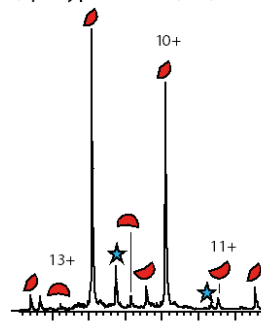

\begin{tabular}{|c|l|c|c|}
\hline & Best Assignment & Experimental mass (Da) & Theoretical mass (Da) \\
\hline $\boldsymbol{\lambda}$ & $\beta$-trypsin & 23296 & 23293 \\
\hline $\boldsymbol{D}$ & $\mathrm{HBH}(165-417)$ & 26429 & 26439 \\
\hline $\boldsymbol{D}$ & $\mathrm{HBH}(25-269)$ & 27598 & 27616 \\
\hline $\boldsymbol{D}$ & $\mathrm{HBH}(25-318)$ & 32336 & 32356 \\
\hline
\end{tabular}

d) $\beta$-trypsin:VHV (1:1)
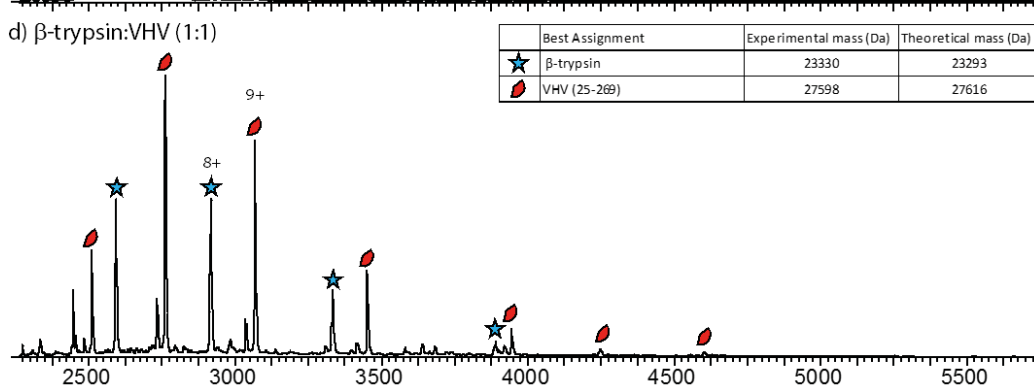

Figure 4.11. Native mass spectra of a) b-trypsin:BHV (1:1), b) b-trypsin:BVB, c) b-trypsin:HBH, d) btrypsin:VHV. In these samples, the proteins were pre-incubated together in PBS after which buffer exchange into AmAc was done.

\subsection{References}

(1) Leader, B., Baca, Q. J., and Golan, D. E. (2008) Protein therapeutics: a summary and pharmacological classification. Nat Rev Drug Discov 7, 21-39.

(2) Chames, P., Van Regenmortel, M., Weiss, E., and Baty, D. (2009) Therapeutic antibodies: successes, limitations and hopes for the future. Br. J. Pharmacol. 157, 220-233. 
(3) Eggel, A., Baumann, M. J., Amstutz, P., Stadler, B. M., and Vogel, M. (2009) DARPins as bispecific receptor antagonists analyzed for immunoglobulin e receptor blockage. J. Mol. Biol. 393, 598-607.

(4) Silverman, J., Lu, Q., Bakker, A., To, W., Duguay, A., Alba, B. M., Smith, R., Rivas, A., Li, P., Le, H., Whitehorn, E., Moore, K. W., Swimmer, C., Perlroth, V., Vogt, M., Kolkman, J., and Stemmer, W. P. C. (2005) Multivalent avimer proteins evolved by exon shuffling of a family of human receptor domains. Nat Biotech 23, 1556-1561.

(5) Baeuerle, P. A., and Reinhardt, C. (2009) Bispecific T-cell engaging antibodies for cancer therapy. Cancer Res. 69, 4941-4944.

(6) Chames, P., and Baty, D. (2009) Bispecific antibodies for cancer therapy: The light at the end of the tunnel? mAbs 1, 539-547.

(7) Müller, D., and Kontermann, R. (2011) Recombinant bispecific antibodies for cancer therapy, in Antibody Expression and Production (Al-Rubeai, M., Ed.), pp 235-249. Springer Netherlands.

(8) Kim, J. W., Cochran, F. V., and Cochran, J. R. (2015) A chemically cross-linked knottin dimer binds integrins with picomolar affinity and inhibits tumor cell migration and proliferation. J. Am. Chem. Soc. 137, 6-9.

(9) Papo, N., Silverman, A. P., Lahti, J. L., and Cochran, J. R. (2011) Antagonistic VEGF variants engineered to simultaneously bind to and inhibit VEGFR2 and $\alpha(v) \beta(3)$ integrin. Proc. Natl. Acad. Sci. U. S. A. 108, 14067-14072.

(10) Cochran, J. R. (2010) Engineered proteins pull double duty. Sci. Transl. Med. 2, 17ps5-17ps5.

(11) Liu, C., and Cochran, J. (2014) Engineering multivalent and multispecific protein therapeutics, in Engineering in Translational Medicine (Cai, W., Ed.), pp 365-396. Springer London.

(12) Dueber, J. E., Wu, G. C., Malmirchegini, G. R., Moon, T. S., Petzold, C. J., Ullal, A. V., Prather, K. L. J., and Keasling, J. D. (2009) Synthetic protein scaffolds provide modular control over metabolic flux. Nat Biotech 27, 753-759.

(13) Liu, F., Banta, S., and Chen, W. (2013) Functional assembly of a multi-enzyme methanol oxidation cascade on a surface-displayed trifunctional scaffold for enhanced NADH production. Chem. Commun. 49, 3766-3768.

(14) Jullesson, D., David, F., Pfleger, B., and Nielsen, J. Impact of synthetic biology and metabolic engineering on industrial production of fine chemicals. Biotechnol. Adv.

(15) Bostrom, J., Yu, S.-F., Kan, D., Appleton, B. A., Lee, C. V., Billeci, K., Man, W., Peale, F., Ross, S., Wiesmann, C., and Fuh, G. (2009) Variants of the antibody herceptin that interact with HER2 andVEGF at the antigen binding site. Science 323, 1610-1614.

(16) Moore, S. J., Leung, C. L., and Cochran, J. R. (2012) Knottins: disulfide-bonded therapeutic and diagnostic peptides. Pept. Modif. Pept. Drug Mol. 9, e3-e11.

(17) Gould, A., Ji, Y., Aboye, T. L., and Camarero, J. A. (2011) Cyclotides, a novel ultrastable polypeptide scaffold for drug discovery. Curr. Pharm. Des. 17, 4294-4307.

(18) Gracy, J., Le-Nguyen, D., Gelly, J.-C., Kaas, Q., Heitz, A., and Chiche, L. (2008) Knottin: the knottin or inhibitor cystine knot scaffold in 2007. Nucleic Acids Res. 36, D314-D319.

(19) Lahti, J. L., Silverman, A. P., and Cochran, J. R. (2009) Interrogating and predicting tolerated sequence diversity in protein folds: application to E. elaterium trypsin inhibitor-II cystine-knot miniprotein. PLoS Comput Biol 5, e1000499.

(20) Christmann, A., Walter, K., Wentzel, A., Krätzner, R., and Kolmar, H. (1999) The cystine knot of a squash-type protease inhibitor as a structural scaffold for Escherichia coli cell surface display of conformationally constrained peptides. Protein Eng. 12, 797-806.

(21) Gunasekera, S., Foley, F. M., Clark, R. J., Sando, L., Fabri, L. J., Craik, D. J., and Daly, N. L. (2008) Engineering stabilized vascular endothelial growth factor-a antagonists: synthesis, 
structural characterization, and bioactivity of grafted analogues of cyclotides. J. Med. Chem. 51, 7697-7704.

(22) Getz, J. A., Rice, J. J., and Daugherty, P. S. (2011) Protease-resistant peptide ligands from a knottin scaffold library. ACS Chem. Biol. 6, 837-844.

(23) Getz, J. A., Cheneval, O., Craik, D. J., and Daugherty, P. S. (2013) Design of a cyclotide antagonist of neuropilin-1 and -2 that potently inhibits endothelial cell migration. ACS Chem. Biol. 8, 1147-1154.

(24) Kimura, R. H., Levin, A. M., Cochran, F. V., and Cochran, J. R. (2009) Engineered cystine knot peptides that bind $\alpha_{v} \beta_{3}, \alpha v \beta_{5}$, and $\alpha_{5} \beta_{1}$ integrins with low-nanomolar affinity. Proteins Struct. Funct. Bioinforma. 77, 359-369.

(25) Heitz, A., Le-Nguyen, D., and Chiche, L. (1999) Min-21 and min-23, the smallest peptides that fold like a cystine-stabilized $\beta$-sheet motif: design, solution structure, and thermal stability. Biochemistry (Mosc.) 38, 10615-10625.

(26) Souriau, C., Chiche, L., Irving, R., and Hudson, P. (2005) New binding specificities derived from min-23, a small cystine-stabilized peptidic scaffold. Biochemistry (Mosc.) 44, 7143-7155.

(27) Chang, H.-J., Hsu, H.-J., Chang, C.-F., Peng, H.-P., Sun, Y.-K., Yu, H.-M., Shih, H.-C., Song, C.-Y., Lin, Y.-T., Chen, C.-C., Wang, C.-H., and Yang, A.-S. (2009) Molecular evolution of cystine-stabilized miniproteins as stable proteinaceous binders. Structure 17, 620-631.

(28) Bode, W., Greyling, H. J., Huber, R., Otlewski, J., and Wilusz, T. (1989) The refined $2.0 \AA$ A X-ray crystal structure of the complex formed between bovine $\beta$-trypsin and CMTI-I, a trypsin inhibitor from squash seeds (Cucurbita maxima) Topological similarity of the squash seed inhibitors with the carboxypeptidase A inhibitor from potatoes. FEBS Lett. 242, 285-292.

(29) Chang, H.-J., Hsu, H.-J., Chang, C.-F., Peng, H.-P., Sun, Y.-K., Yu, H.-M., Shih, H.-C., Song, C.-Y., Lin, Y.-T., Chen, C.-C., Wang, C.-H., and Yang, A.-S. (2009) Molecular evolution of cystine-stabilized miniproteins as stable proteinaceous binders. Structure 17, 620-631.

(30) Grzesiek, S., Bax, A., Hu, J.-S., Tjandra, N., Kaufman, J., Palmer, I., Stahl, S. J., and Wingfield, P. T. (1997) Refined solution structure and backbone dynamics of HIV-1 Nef. Protein Sci. 6, 12481263.

(31) Muller, Y. A., Li, B., Christinger, H. W., Wells, J. A., Cunningham, B. C., and de Vos, A. M. (1997) Vascular endothelial growth factor: Crystal structure and functional mapping of the kinase domain receptor binding site. Proc. Natl. Acad. Sci. U. S. A. 94, 7192-7197.

(32) Reddy Chichili, V. P., Kumar, V., and Sivaraman, J. (2013) Linkers in the structural biology of protein-protein interactions. Protein Sci. Publ. Protein Soc. 22, 153-167.

(33) Chen, X., Zaro, J. L., and Shen, W.-C. (2013) Fusion protein linkers: Property, design and functionality. Ed. Collect. $201365,1357-1369$.

(34) Schmoldt, H.-U., Wentzel, A., Becker, S., and Kolmar, H. (2005) A fusion protein system for the recombinant production of short disulfide bond rich cystine knot peptides using barnase as a purification handle. Protein Expr. Purif. 39, 82-89.

(35) Banerjee, S., and Mazumdar, S. (2012) Electrospray ionization mass spectrometry: a technique to access the information beyond the molecular weight of the analyte. Int. J. Anal. Chem. 2012.

(36) Morrison, J. F. (1969) Kinetics of the reversible inhibition of enzyme-catalysed reactions by tight-binding inhibitors. Biochim. Biophys. Acta BBA - Enzymol. 185, 269-286.

(37) Rurup, W. F., Verbij, F., Koay, M. S. T., Blum, C., Subramaniam, V., and Cornelissen, J. J. L. M. (2014) Predicting the loading of virus-like particles with fluorescent proteins. Biomacromolecules 15, 558-563. 


\section{Chapter 5}

\section{Optical control over bioactive ligands at supramolecular surfaces}

In this chapter azobenzene modified glycoconjugates are used to establish an optically controllable platform to present bioactive ligands at a supramolecular B-cyclodextrin (B$C D$ ) surface. The binding affinity of an azobenzene glycoconjugate on $B-C D$ self-assembled monolayers (SAMs) was determined using quartz crystal microbalance (QCM) measurements and differences between the binding of trans and cis forms of azobenzene were also observed. The photoresponsive immobilization of a lectin, Conconavalin $A$ (ConA) and E. coli on these supramolecular surfaces was evaluated through QCM measurements and using microcontact printed arrays of azobenzene compounds on B-CD SAMs. This work provides insights into the possibility of developing responsive surfaces for biomedical applications where biological entities can be immobilized and released under mild conditions.

This work in this chapter was done along with Dr. Jens Voskuhl and is part of the communication: Voskuhl, J.," Sankaran, S.," and Jonkheijm, P. (2014) Optical control over bioactive ligands at supramolecular surfaces. Chem. Commun. 50, 15144-15147. \# Equal Authorship 


\subsection{Introduction}

Dynamic arrays of bioactive ligands have recently attracted much interest since they enable real-time control over surface properties by employing external and remote triggers. ${ }^{1-3}$ While arrays of biomolecules have been applied in various areas of biomedical and bioanalytical research, ${ }^{4}$ stimuli responsive behaviour of these surfaces can easily be offered by supramolecular chemistry. Employing supramolecular chemistry not only delivers improved specificity, directionality and tunability of the interaction motifs and strengths but responsiveness of the presentation of bioactive ligands at surfaces can be achieved as well. ${ }^{5}$

Supramolecular bioactive surfaces that employ host molecules, such as cucurbit[n]urils $(C B[n])^{6-8}$ and cyclodextrins $(C D), 9,10$ have been recently employed. These cyclic structures have the ability to encapsulate hydrophobic bulky guest molecules such as adamantane and ferrocene. Approaches to fabricate such supramolecular bioactive platforms involve attaching the host molecules directly to the surface by using covalent silane chemistry on glass, ${ }^{11,12}$ thiol chemistry on gold ${ }^{13,14}$ or in the case of CBs, even coordinative chemistry between gold surfaces and the glycouril oxygens has been reported. ${ }^{15,16}$ For example, we supramolecularly printed proteins bearing a ferrocene unit on $\beta-C D$ monolayers to achieve electrochemical responsive protein arrays. ${ }^{9,17}$ Ferrocene, which binds as neutral species to $\beta-C D$, but does not in the oxidized state, was erased from printed arrays following an external electrochemical stimulus. ${ }^{9}$ Alternatively, non-covalent strategies can be employed by fixing the guest molecules to the surface and allowing the host to bind from solution. ${ }^{18}$ This strategy has been successfully utilized in the case of electro-responsive peptide surfaces based on $\mathrm{CB}[8]^{7,8,19}$ and some $\mathrm{CD}$ based systems. ${ }^{20}$ Onto this type of supramolecular surfaces, we supramolecularly attached napthol-modified fluorescent proteins and tryptophanmodified RGD peptides modified biomolecules on methylviologen monolayers in the presence of $\mathrm{CB}[8]]^{7,21}$ These latter surfaces were used for the supramolecular detachment of cells when RGD peptides were used following an external reductive potential.7 Although redox-responsive supramolecular bioactive surfaces have been demonstrated to have important biomimetic potentialities, reports on photoresponsive CD based surface systems are scarce. ${ }^{22}$ Photosensitive host-guest complexes are of significant interest as light can be applied in a remote manner as an external stimulus and offers precise control over wavelength.

Here we report a novel approach on the use of highly ordered monolayers of $\beta-C D$ on gold and glass surfaces to immobilize glycoconjugates via a photoresponsive 108 
azobenzene moiety (Figure 5.1). Azobenzenes are well known molecules in the field of supramolecular chemistry since they bear the opportunity to bind to CDs and CBs in the stable trans form and can be released from the cavity after UV-irradiation by conversion into the bulkier, more hydrophilic cis-azobenzene. ${ }^{1,23-25}$ Although this behaviour has been successfully used in previous studies to fabricate photoresponsive supramolecular nanoparticles and polymers based on $\mathrm{CB}[8]^{26-28}$ as well as light responsive systems consisting of amphiphilic CDs to induce aggregation, ${ }^{29}$ to transport DNA and proteins $\mathrm{s}^{30,31}$ and to tune the wettability ${ }^{32,33}$ and to modulate the assembly of cells ${ }^{22}$ and gels, ${ }^{34}$ no previous report has demonstrated optical control over protein and bacterial immobilization on $\beta-C D$ modified surfaces. In this chapter, we address whole protein and bacterial immobilization and their optical control on the supramolecular platform, which is significantly more complex than previous photoresponsive supramolecular studies in solutions.

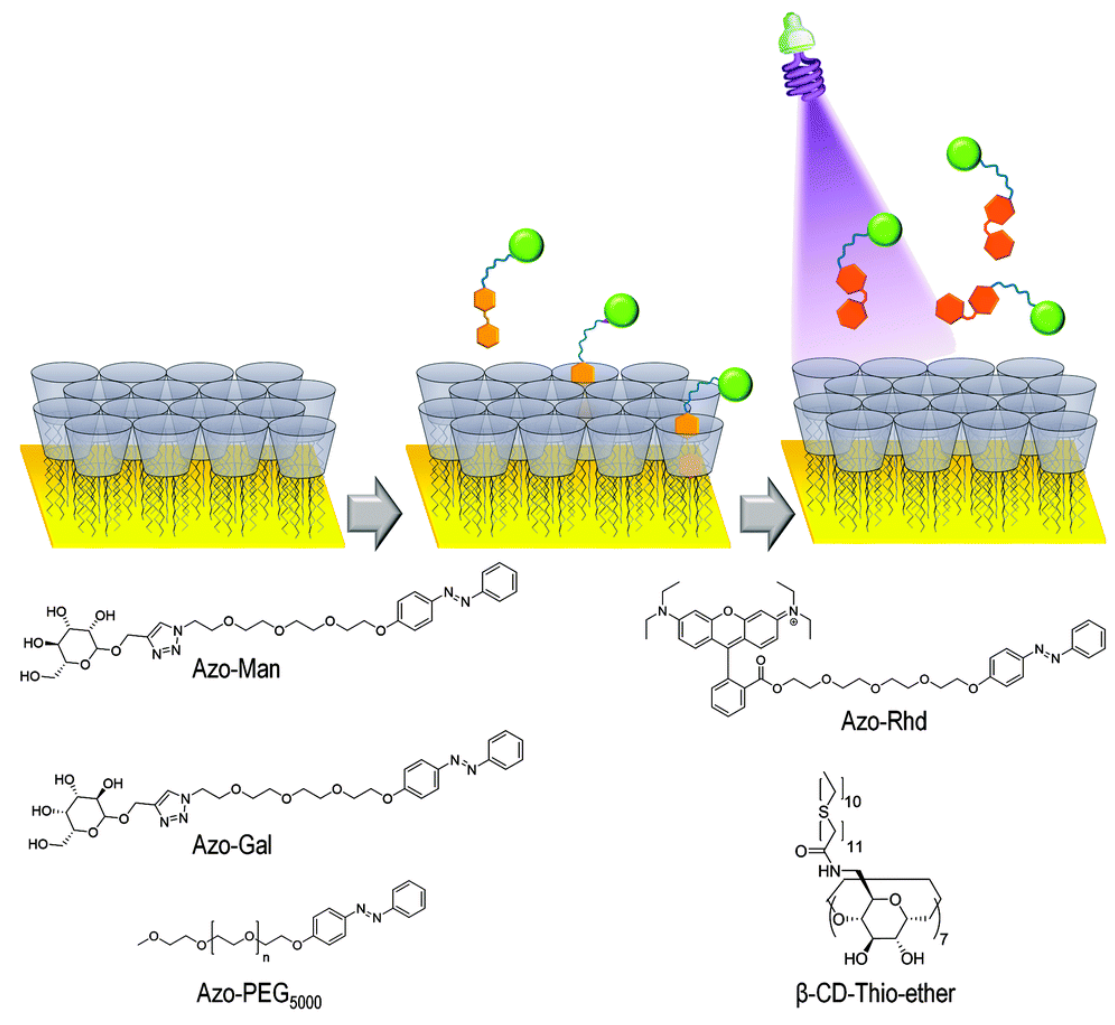

Figure 5.1. Assembly of bioactive ligands with photoswitchable properties on a b-cyclodextrin monolayer. Given molecular structures are used in this chapter. 


\subsection{Results and Discussions}

\subsubsection{QCM Characterization}

Herein, two glycoconjugates were synthesized bearing an azobenzene unit linked via a tetraethyleneglycol unit to either mannose or galactose (Figure 5.1). Mannose is known to bind carbohydrate specific lectins such as concanavalin A (ConA) and the well-known FimH receptor on pathogenic bacteria like $E$. coli. Furthermore, a fluorescently labelled azobenzene derivative bearing a rhodamine unit was synthesized (Figure 5.1, Azo-Rhd) to visualize azobenzenes assembled onto $\beta-C D$ surfaces. To measure the binding affinity of azobenzenes to the $\beta-C D$ monolayer on gold, a titration series of Azo-Man over a range of $25 \mu \mathrm{M}-1 \mathrm{mM}$ was performed using quartz crystal microbalance (QCM) at a flow rate of $100 \mu \mathrm{L} \mathrm{min}{ }^{-1}$ followed by extensive washing with buffer (Figure 5.2A). Binding events caused a drop in the resonance frequency of the crystal and the 5 th harmonic was followed as it presented the best signal to noise ratio. In all QCM studies performed dissipation was also monitored, but the change appeared negligible. In order to determine the association constant, the values of the changes in frequency at saturation were plotted against concentrations of Azo-Man. Fitting using the Langmuir equation yielded a $K_{a}$ value of $5.8 \times 10^{3} \mathrm{M}^{-1}$ for binding of Azo-Man onto $\beta-C D$ monolayers (Figure 5.2B). This value favourably compares to the binding affinity of azobenzene to $\beta-C D$ in solution, which was previously determined by isothermal titration calorimetry (ITC) in aqueous media $\left(K_{a}=2.4 \times 10^{3} \mathrm{M}^{-1}\right) .^{30,31}$ With this knowledge, the difference in binding between cis and trans-forms of Azo-Man (Figure $5.2 \mathrm{E}$ ) on the $\beta-C D$ monolayer was investigated. To this end, a $250 \mu \mathrm{M}$ solution of AzoMan was irradiated with UV light $(\lambda=365 \mathrm{~nm})$ for 10 min prior to injection in the flow chamber. As expected, a significant difference in frequency change was observed between the cis-and trans-forms of Azo-Man, signifying a lower affinity of the cis-Azo to the $\beta$-CD SAM. Since the photoisomerization efficiency is about $80 \%$ as determined from ${ }^{1} \mathrm{H}$-NMR studies, ${ }^{30}$ (Figure S5.1, Section 5.7, Supporting Information) about $50 \mu \mathrm{M}$ still remains in the trans-form which nicely accounts for the observed residual change in frequency signal of -6 (Figure $5.2 \mathrm{C}$ ). In a final QCM experiment, the binding of ConA onto $\beta$-CD SAMs bearing carbohydrate Azo-Man was investigated and using Azo-Gal as negative control. Flowing $1 \mathrm{mM}$ solutions of glycoconjugates over a $\beta$-CD SAM on gold, led to a significant change in frequency similar to what was seen in the previous measurements. Subsequently, a $50 \mu \mathrm{g} \mathrm{mL} \mathrm{L}^{-1}$ solution of ConA was led over the carbohydrate bearing surfaces. In the case of the Azo-Man surface, this caused the frequency to dramatically change, corresponding to the adsorption of ConA onto this 
surface (Figure 5.2D). The Azo-Gal control surface exhibited a much smaller QCM response showing that ConA binding occurs specifically to mannose. Washing with buffer resulted in dissociation of ConA, which occurred at a reduced rate compared to the association to Azo-Man. This observation is probably related to the tetrameric nature of ConA that could bind two mannose units on a flat surface. Consequently, this causes it to be able to bind to Azo-Man at the $\beta-C D$ monolayer in a bivalent manner. Washing with an excess of (D)-mannose leads to a higher dissociation rate of the proteins from the Azo-Man surface, supporting the fact that binding occurs specifically.
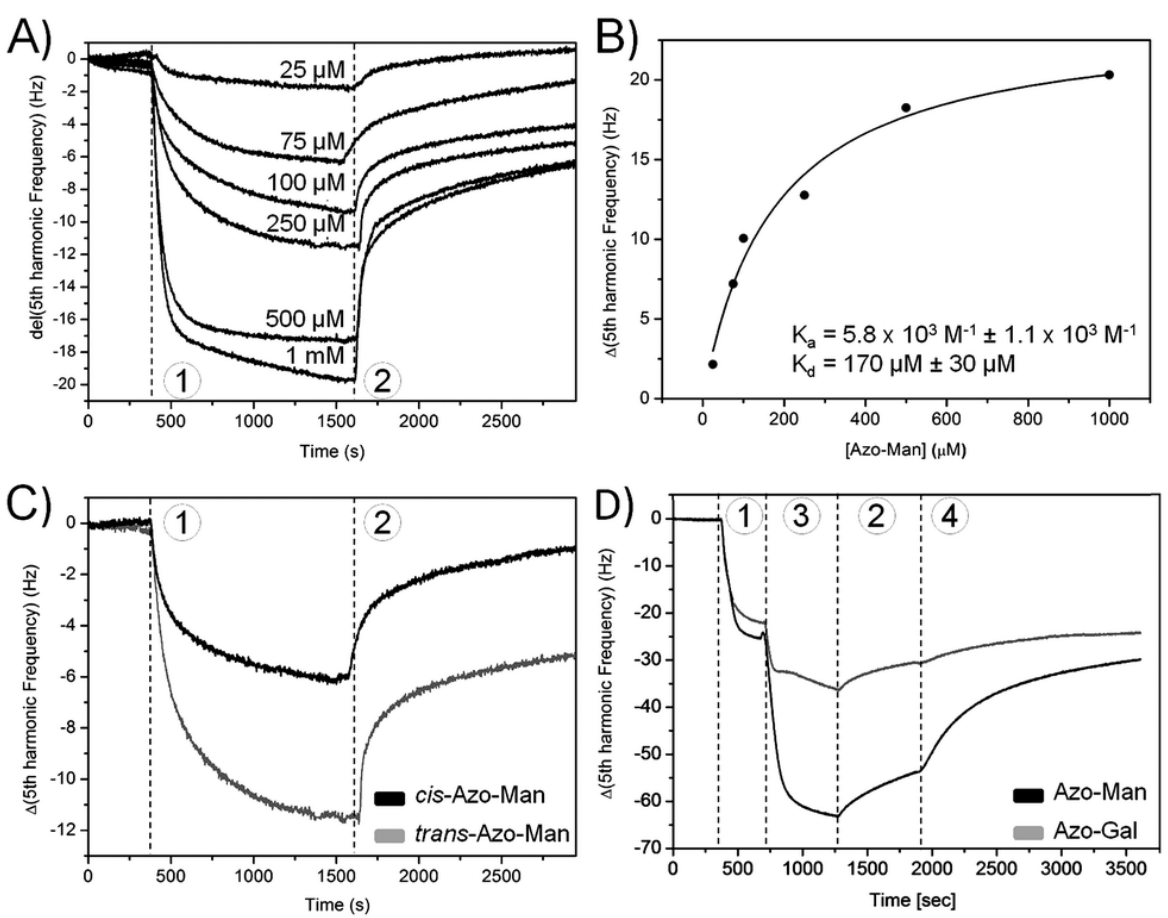

E)

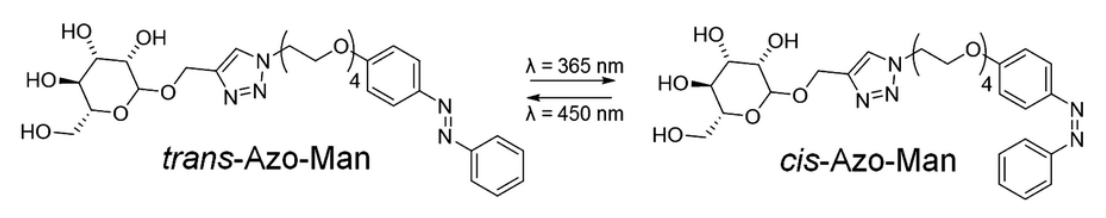

Figure 5.2. (A) QCM measurements of Azo-Man at varying concentrations on a B-CD-SAM. (B) Binding curve of Azo-Man to B-CD-SAM. (C) Differences in QCM response between cis-Azo-Man and trans-Azo-Man on a B-CD-SAM. (D) Immobilization of ConA onto Azo-Man and Azo-Gal at B-CD-SAM. Legends: (1) incubation with Azo-Man or Azo-Gal, (2) washing with PBS, (3) incubation with ConA, (4) washing with (D)-mannose. (E) trans-cis isomerisation of Azo-Man. 


\subsubsection{Microcontact printing}

To visualize the optical control over the assembly of bioactive molecules, microcontact printing was performed on $\beta-C D$ modified glass substrates and imaged using epifluorescence microscopy. In a first experiment, a $500 \mu \mathrm{M}$ Azo-Man solution mixed with $2.5 \mu \mathrm{M}$ Azo-Rhd was printed using a PDMS-stamp. Following brief washing with water patterns of dots that are $100 \mu \mathrm{m}$ in diameter with a spacing of $100 \mu \mathrm{m}$ were clearly observed, as seen in Figure 5.3A, indicating the successful immobilization of the inked molecule onto the $\beta-C D$ surfaces. Next, Azo-Man was printed in lines on the $\beta-C D$

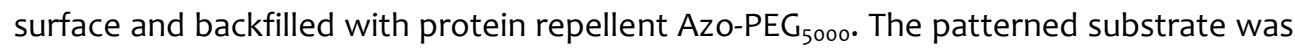
then incubated with a fluorescein-conjugated ConA and washed briefly. This experiment produced clear patterns of immobilized ConA indicating the successful interplay of two orthogonal supramolecular interactions on the $\beta$-CD surface (Figure 5.3B). As a next step, the suitability of these supramolecular Azo-Man surfaces for binding of bacterial cells was investigated. Two different strains of E. coli were selected that differ only in their mannose binding properties to assess whether the mannosefunctionalized supramolecular surfaces can interact with $E$. coli. In addition to a strain that binds to mannose (ORN-178), a second strain (ORN-208) was used where its FimH receptor was mutated to eliminate mannose binding. In a first experiment, two $\beta-C D$ surfaces with immobilized Azo-Man were separately incubated with the two bacterial strains. Both substrates were then incubated with Hoechst $\mathrm{H} 33342$ to stain the DNA in the bacteria. Representative images are shown in (Figure 5.3C \& 5.3D). From these images it can be seen that only the $\mathrm{ORN}-178$ strain was able to successfully interact with the surface. After washing the bacterial cells remained stably bound at the surface. In the case of ORN-208 a negligible number of surface-immobilized bacterial cells were observed, confirming the selectivity of the supramolecular system. Finally, we printed the Azo-Man in line patterns and backfilled the interspace with Azo-PEG ${ }_{5000}$ to avoid non-specific interactions. After DNA staining of ORN-178, clear line patterns of blue bacteria were observable (Figure 5.3E) showing the possibility to assemble bacteria in patterns on Azo-Man surfaces. 

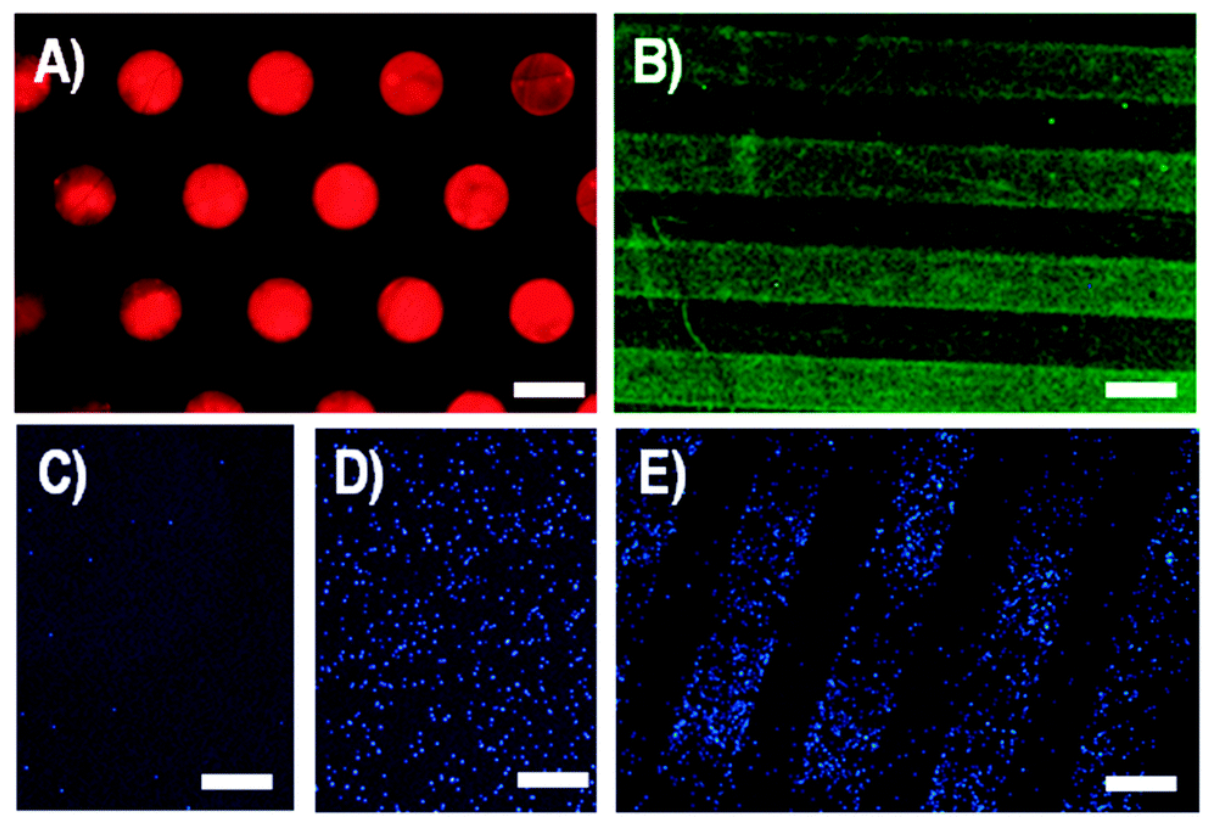

Figure 5.3. Fluorescence microscopy images of surfaces where (A) Azo-Man/Azo-Rhd (200/1) was printed on a B-CD SAM, Azo-Man was assembled on B-CD SAMs followed by incubation with (B) fluorescein-conjugated ConA, (C) E. coli strain ORN-208, (D) and (E) E. coli strain ORN-178. The scale bars represent $100 \mu \mathrm{m}$.

The binding of the bacteria to Azo-Man was also confirmed using QCM (Figure 5.4). A $\beta$ CD SAM on gold was initially exposed to a solution of Azo-Man $(500 \mu \mathrm{M})$ followed by flowing of ORN178 (in the presence of Azo-Man), which produced a clear change in frequency, which remained unchanged on switching to Azo-Man only. Changing to a flow of buffer and subsequently to a solution of $\beta-C D$ showed a change in frequency that is indicative of competitive binding of the cyclodextrin in solution with surface bound azobenzene. 


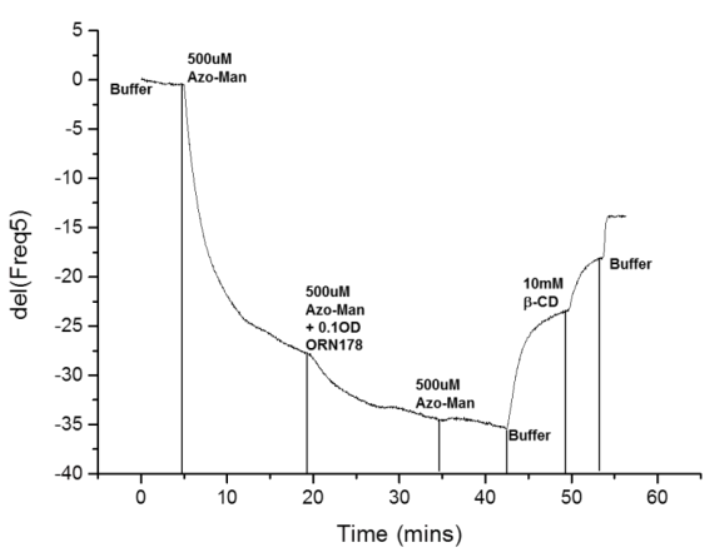

(a)

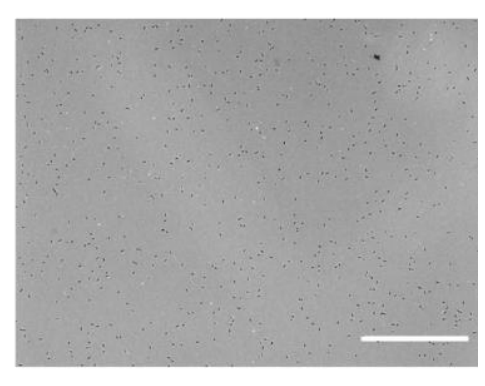

(b)

Figure 5.4. (a) QCM response curve ( $\triangle$ Frequency of $5^{\text {th }}$ harmonic) for bacterial surface immobilization. (b) Representative area on sensor chip after all measurements were visualized under a microscope in bright field mode. Bacteria are seen as small black rods. Scale bar corresponds to $100 \mu \mathrm{m}$.

\subsubsection{Photo-responsiveness}

To assess the photo-responsiveness of the supramolecular system, patterns of AzoMan mixed with $1 \%$ Azo-Rhd were printed on two $\beta-C D$ modified glass substrates and imaged with a fluorescence microscope. Both surfaces were then placed in water for 5 min with one substrate exposed to $365 \mathrm{~nm}$ UV light while the other substrate was kept in the dark. Fluorescence images were again recorded (Figure 5.5 A) and intensity profiles were made and averaged over a $7 \mathrm{~mm}^{2}$ area and plotted as percentage of the intensity prior to washing (Figure $5.5 \mathrm{C}$ ). The substrate that was not irradiated with the $365 \mathrm{~nm}$ light retained nearly $50 \%$ of its original fluorescence intensity, which corresponds to the dissociation as observed in our QCM experiments. In strong contrast, the substrate exposed to the UV irradiation, lost almost all its original fluorescence intensity, which can be attributed to the photoisomerization of the azobenzene units. To extend the photo-control of the supramolecular system towards ConA protein assembly, a similar experiment was conducted where printed Azo-Man was released from a $\beta-C D$ surface using UV irradiation and backfilled with Azo-PEG $G_{5000}$. A non-irradiated substrate was used as comparison. Fluorescein-conjugated ConA (ConA-FAM) was then allowed to interact with these surfaces. Fluorescence microscopy images revealed clear line patterns on the non-irradiated surface and very 
faint patterns under high exposure conditions on the irradiated surface (Figure 5.5B \& 5.5D). Optical release of a ConA-Cy3 conjugate bound to Azo-Man patterns on $\beta-C D$ surfaces was tested (Figure S5.2, Section 5.7, Supporting Information). UV irradiation caused dissociation in the ConA-Cy3 from the Azo-Man patterns, however washing and incubation steps seemed to have resulted in diffusion of ConA-Cy3 over the whole surface.
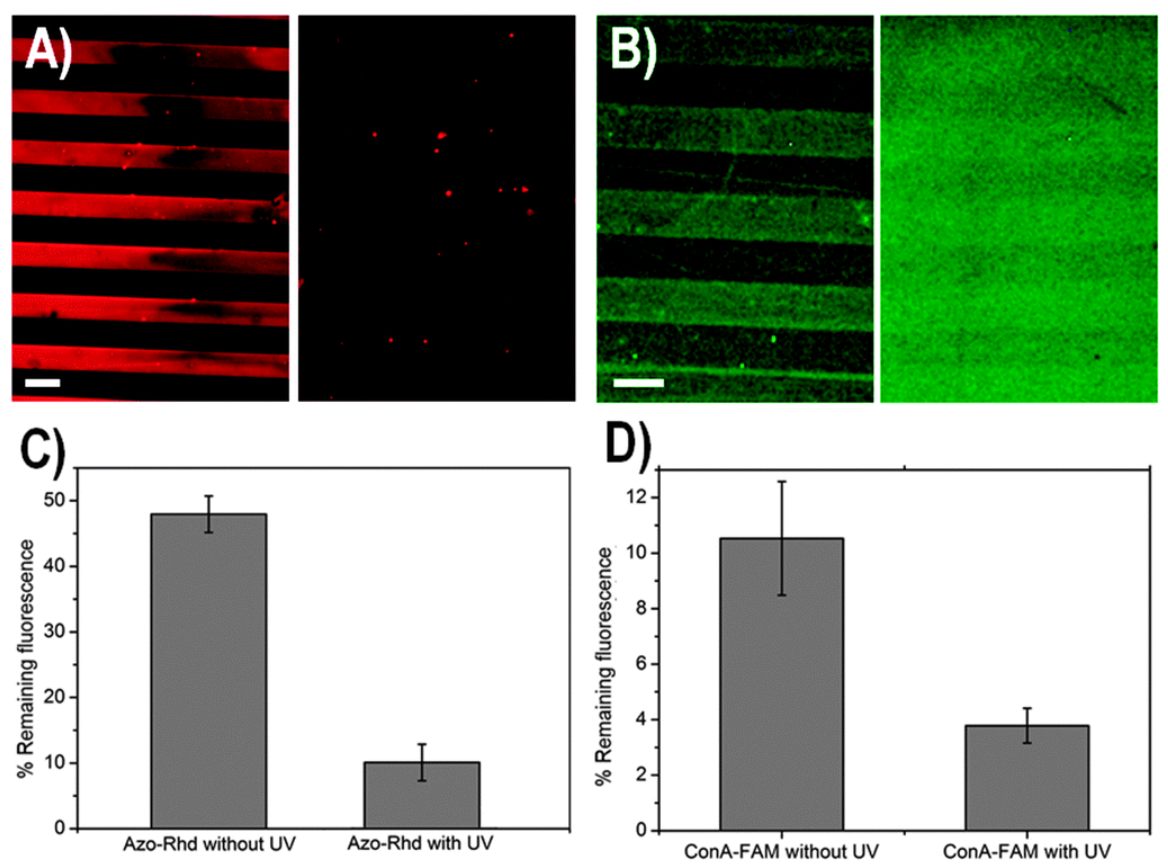

Figure 5.5. (A) Fluorescence microscopy image of Azo-Rhd immobilized on CD-SAMs before and after irradiation with UV-light. (B) Fluorescein labeled ConA-FAM immobilized on Azo-Man before and after irradiation with UV light. (C) Intensity profile of Azo-Rhd before and after irradiation with UV light. (D) Intensity profile of ConA-FAM immobilized on Azo-Man before and before and after irradiation with UV light. Scale bars represent $100 \mu \mathrm{m}$.

\subsection{Conclusions}

We have described a supramolecular approach for achieving for the first time supramolecular optical control of biomolecules by an external light stimulus. We were able to successfully determine the binding affinity of glycoconjugates on $\beta$-cyclodextrin SAMs by QCM measurements. Furthermore, several fluorescence microscopy images showed the effective immobilization of azobenzene functionalized dyes and 
carbohydrates that can specifically interact with proteins and bacteria. Furthermore it was shown that azobenzene molecules can be released by an external light stimulus and modulate the assembly of ConA proteins. Taken into account that larger biological entities, such as bacteria, have about $200 \mathrm{FimH}$ receptors on its membrane surface and that ca. $20 \%$ of the azobenzene moieties that has been used in this study remain in its trans-conformation after UV-light irradiation, a significant number of receptors would hold the binding of bacteria to the substrate. However, further optimization of the azobenzene modification, linker lengths and surface assembly strategy will broaden possible applications of our work. The current work bears the potential for further purposes such as analysis of surface bound proteins by ligands which can be removed under mild conditions.

\subsection{Acknowledgements}

Carmen Stoffelen is acknowledged for supply of the azobenzene-polyethylene conjugate. ORN strains were kindly provided by Prof. L. Brunsveld.

\subsection{Experimental Section}

Materials. Chemicals were purchased from Sigma Aldrich or from Acros Organics and used without further purification. Reactions were carried out using dried solvent and under an atmosphere of argon. Reactions were monitored by thin-layer chromatography (TLC), which was performed on $0.2 \mathrm{~mm}$ Merck precoated silica gel 60 F254 aluminum sheets. Spots were visualized by treatment with basic $\mathrm{KMnO}_{4}$ solution. Column chromatography was carried out on silica gel 60 (0.063-0.2 mm, Merck). NMR spectra were recorded on Bruker spectrometers (AV400). Chemical shifts are given in units of parts per million (ppm) and expressed relative to the signals of deuterated solvents. Coupling constants $(J)$ are reported in Hertz $(\mathrm{Hz})$. Mass spectra were recorded with a MicroToF spectrometer (Bruker). 
Synthesis of the azobenzene-carbohydrate conjugates.

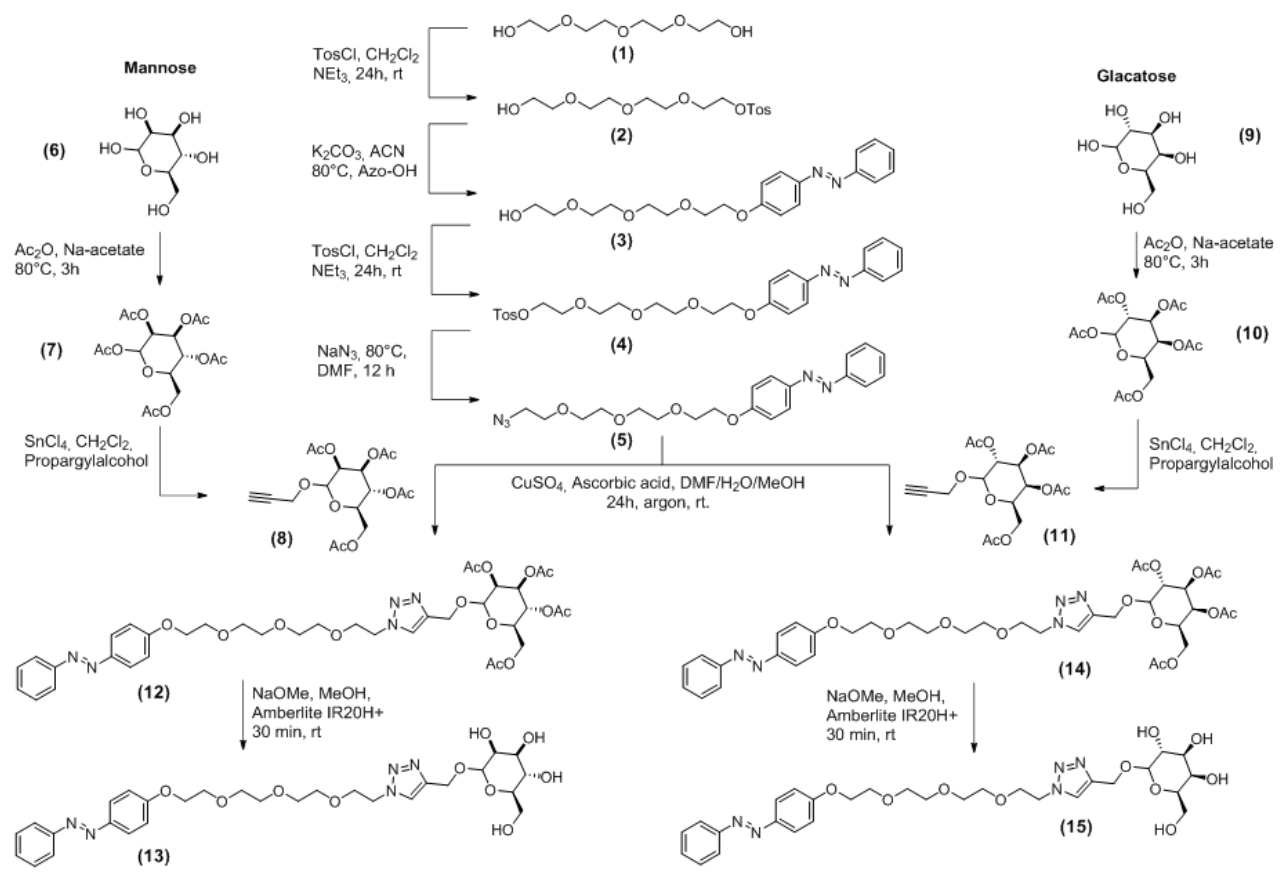

Figure 5.6. Synthesis route to the azobenzene substituted glycosides.

The synthesis of compound $\mathbf{2 , 3}, \mathbf{4}, \mathbf{7}, \mathbf{8 ,} 10$ and $\mathbf{1 1}$ was carried out according to a known literature procedure. ${ }^{35,36}$

2-(2-(2-hydroxyethoxy)ethoxy)ethyl 4-methylbenzenesulfonate $(2)^{36}$

Toso $130.0 \mathrm{~g}, 154 \mathrm{mmol}, 1 \mathrm{eq}$.) in catalytic amount of DMAP (50 mg). The mixture was stirred for 30 minutes. After that time p-toluenesulfonylchloride was added portionwise (7.24 g, $39 \mathrm{mmol}, 1 \mathrm{eq}$.). The reaction mixture was stirred overnight followed by evaporation of the solvent. The residue was redissolved in chloroform and extracted three times with water. The organic layer was separated and dried over $\mathrm{MgSO}_{4}$. The solvent was removed under reduced pressure. The title compound was obtained as colorless oil. Yield: $9.51 \mathrm{~g}, 70 \%$ ${ }^{1} \mathrm{H}$ NMR (300 MHz, CDCl3, $\left.298 \mathrm{~K}\right): \delta=2.37(\mathrm{~s}, 3 \mathrm{H}, 1-\mathrm{H}), 3.44-3.51(\mathrm{~m}, 10 \mathrm{H}, 8-11-\mathrm{H}), 3.61(\mathrm{t}$, $2 \mathrm{H}, 7-\mathrm{H}), 4.09(\mathrm{t}, 2 \mathrm{H}, 6-\mathrm{H}), 7.27(\mathrm{~d}, J=6.0 \mathrm{~Hz}, 2 \mathrm{H}, 3-\mathrm{H}), 7.73(\mathrm{~d}, J=6.0 \mathrm{~Hz}, 2 \mathrm{H}, 4-\mathrm{H})$. ESI- 
MS (m/z): calculated for $\left[\mathrm{C}_{13} \mathrm{H}_{20} \mathrm{O}_{6} \mathrm{SNa}\right]^{+}: 329.1814$, found: 329.1911. The spectroscopic data are in agreement with those reported in literature.

(E)-2-(2-(2-(2-(4-(phenyldiazenyl)phenoxy)ethoxy)ethoxy)ethoxy)ethanol (3) 30

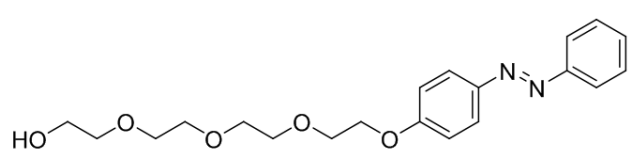

To a stirred solution of (2) $(5.23 \mathrm{~g}, 15$ $\mathrm{mmol}$ ) in $150 \mathrm{ml}$ of dry acetonitrile, containing of $\mathrm{K}_{2} \mathrm{CO}_{3}(10.37 \mathrm{~g}, 75 \mathrm{mmol})$ and catalytic amounts of $\mathrm{LiBr}$, (3.17 $\mathrm{g} 16$ $\mathrm{mmol}$ ) of 4-phenylazophenol dissolved in $50 \mathrm{ml}$ of acetonitrile was added and the reaction mixture was refluxed for 2 days under argon. It was then allowed to cool to room temperature and the solvent was removed under reduced pressure. The residue was dissolved in $100 \mathrm{ml}$ of DCM, washed once with $100 \mathrm{ml}$ of water and three times with $100 \mathrm{ml}$ of brine. The organic phase was dried over $\mathrm{MgSO}_{4}$ and concentrated. The residue was purified by silica gel column chromatography (DCM / methanol 95:5) to afford the title compound. Yield: $5.11 \mathrm{~g}, 91 \%$. ${ }^{1} \mathrm{H}$ NMR (300 $\mathrm{MHz}, \mathrm{CDCl}_{3}, 298 \mathrm{~K}$ ): $\delta=2.57$ $(\mathrm{s}, 1 \mathrm{H}, 15-\mathrm{H}), 3.58-3.50(\mathrm{~m}, 2 \mathrm{H}, 14-\mathrm{H}), 3.72-3.61(\mathrm{~m}, 10 \mathrm{H}, 11,12,13-\mathrm{H}), 3.83(\mathrm{~m}, 2 \mathrm{H}, 10-\mathrm{H})$, $4.16(\mathrm{~m}, 2 \mathrm{H}, 9-\mathrm{H}), 7.03-6.95(\mathrm{~m}, 2 \mathrm{H}, 10,11-\mathrm{H}), 7.50-7.35(\mathrm{~m}, 3 \mathrm{H}, 1,2,-\mathrm{H}), 7.92-7.80(\mathrm{~m}$, $4 \mathrm{H}, 3,6-\mathrm{H})$. The spectroscopic data are in agreement with those reported in literature

(E)-2-(2-(2-(4-(phenyldiazenyl)phenoxy)ethoxy)ethoxy)ethoxy)ethyl-4methylbenzensulfonate (4)

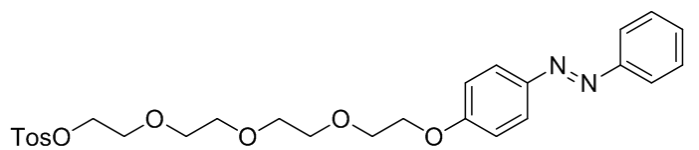

To a stirred solution of (3) (3.00 g, 10 mmol, 1 eq.) in $\mathrm{CH}_{2} \mathrm{Cl}_{2}$ was added $\mathrm{NEt}_{3}$ $(1,2 \mathrm{~mL}, 10 \mathrm{mmol}, 1$ eq. $)$ and a catalytic amount of DMAP (50 mg). The mixture was stirred for 30 minutes. After that time p-toluenesulfonylchloride was added portionwise $(2.07 \mathrm{~g}, 10 \mathrm{mmol}, 1 \mathrm{eq}$.). The reaction mixture was stirred overnight followed by evaporation of the solvent. The residue was redissolved in chloroform and extracted 3 times with water. The organic layer was separated and dried over $\mathrm{MgSO}_{4}$. The solvent was removed under reduced pressure. The residue was subjected to silica column chromatography $\left(\mathrm{CHCl}_{3}\right)$. The product was obtained as orange oil. Yield: $1,78 \mathrm{~g}$, 33 \%. ' $\mathrm{H}-\mathrm{NMR}$ (400 $\mathrm{MHz} \mathrm{CDCl}_{3}$ ): 7.89-7.84 (m, 4H, $4 \mathrm{CH}$ ); 7.76-7.74 (d, $2 \mathrm{H}, 2 \mathrm{CH}$ ); 7.48-7.45 (m, $2 \mathrm{H}, 2 \mathrm{CH}) ; 7.42-7.40(\mathrm{~m}, 1 \mathrm{H}, \mathrm{CH}) ; 7.29-7.27(\mathrm{~m}, 2 \mathrm{H}, 2 \mathrm{CH}) ; 7.00-6.98(\mathrm{~m}, 2 \mathrm{H}, 2 \mathrm{CH}) ; 4.18$ $4.16\left(\mathrm{~m}, 2 \mathrm{H}, \mathrm{CH}_{2}\right)$; 4.13-4.10 (m, 2H, $\left.\mathrm{CH}_{2}\right) ; 3.86-3.83\left(\mathrm{~m}, 2 \mathrm{H}, \mathrm{CH}_{2}\right) ; 3.68-3.54\left(\mathrm{~m}, 10 \mathrm{H}, 5 \mathrm{CH}_{2}\right)$; $2.37\left(\mathrm{~s}, 3 \mathrm{H}, \mathrm{CH}_{3}\right)$. ESI-MS (m/z): calculated for $\left[\mathrm{C}_{27} \mathrm{H}_{32} \mathrm{~N}_{2} \mathrm{O}_{7} \mathrm{SH}\right]^{+}$: 529.1930, found: 529.1976 . 
(E)-2-(2-(2-(4-(phenyldiazenyl)phenoxy)ethoxy)ethoxy)ethoxy)ethyl-azide (5)

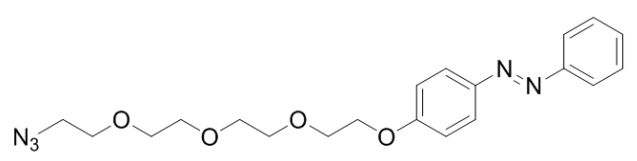

To a stirred solution of (4) $(150 \mathrm{mg}, 0.28$ $\mathrm{mmol}, 1 \mathrm{eq})$ in $10 \mathrm{~mL}$ of DMF was added $\mathrm{NaN}_{3}$ (100 mg, $1.51 \mathrm{mmol}, 5.4$ eq.) and the suspension was heated to $80^{\circ} \mathrm{C}$ and stirred overnight. After that, the solvent was removed in vacuo and the crude residue was dissolved in $20 \mathrm{~mL}$ of $\mathrm{CHCl}_{3}$ and washed three times with $20 \mathrm{~mL}$ of distilled water. The organic layer was collected and dried over $\mathrm{MgSO}_{4}$. The solvent was evaporated and the title compound was obtained as orange-brown oil. Yield: $103 \mathrm{mg}, 91 \%$. 'H-NMR (400 MHz, $\mathrm{CDCl}_{3}$ ): 7.90-7.86 (m, 4H, $\left.4 \mathrm{CH}\right) ; 7.46-7.43(\mathrm{~m}, 2 \mathrm{H}, 2 \mathrm{CH}) ; 7.43-7.41(\mathrm{~m}, 1 \mathrm{H}, \mathrm{CH}) ; 7.02-$ $7.00(\mathrm{~m}, 2 \mathrm{H}, 2 \mathrm{CH}) ; 4.21-4.18\left(\mathrm{t}, 2 \mathrm{H}, \mathrm{J}=4.6 \mathrm{~Hz} \mathrm{CH}_{2}\right) ; 3.89-3.86\left(\mathrm{t}, 2 \mathrm{H}, \mathrm{J}=4.6 \mathrm{~Hz}, \mathrm{CH}_{2}\right) ; 3.72-$ $3.63\left(\mathrm{~m}, 10 \mathrm{H}, 5 \mathrm{CH}_{2}\right) ; 3.43-3.34\left(\mathrm{t}, 2 \mathrm{H}, J=4.6 \mathrm{~Hz}, \mathrm{CH}_{2}\right)$. ESI-MS (m/z): calculated for $\left[\mathrm{C}_{20} \mathrm{H}_{25} \mathrm{~N}_{5} \mathrm{O}_{4} \mathrm{H}\right]^{+}: 400,1907$ found: 400.0972 .

(E)-2-(2-(2-(4-(phenyldiazenyl)phenoxy)ethoxy)ethoxy)ethoxy)ethyl-triazoylperacetylmannopyranoside (12)

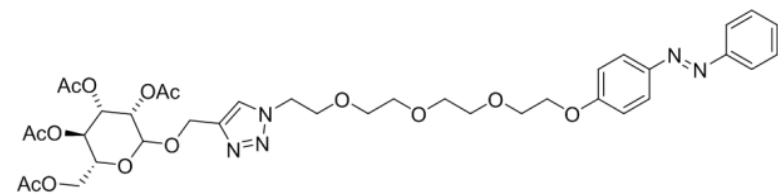

To a mixture of $(5)(50 \mathrm{mg})$ and (8) $(50 \mathrm{mg})$ in a degassed solvent mixture of $\mathrm{MeOH}$, DMF and water (1:2:1) was added sodium ascorbate $(20 \mathrm{mg})$ and $\mathrm{CuSO}_{4}(10 \mathrm{mg})$. The suspension was stirred for $24 \mathrm{~h}$ under an atmosphere of argon followed by evaporation of all solvents. The residue was dissolved in $\mathrm{CHCl}_{3}(1 \mathrm{~mL})$ and subjected to silica column chromatography $\left(\mathrm{CHCl}_{3} / \mathrm{MeOH}\right.$, 9:1). The product was obtained as brown-orange oil. Yield: $91 \mathrm{mg}, 92 \%{ }^{1} \mathrm{H}-\mathrm{NMR}$ (400 $\left.\mathbf{M H z}, \mathrm{CDCl}_{3}\right)$ : 7.87-7.81 (m, 4H, 4CH); $7.77\left(\mathrm{~s}, 1 \mathrm{H}, \mathrm{CH}_{\text {triazole }}\right) ; 7.47-7.43(\mathrm{~m}, 2 \mathrm{H}, 2 \mathrm{CH}) ; 7.41-$ $7.37(\mathrm{~m}, 1 \mathrm{H}, \mathrm{CH}) ; 6.99-6.96(\mathrm{~m}, 2 \mathrm{H}, 2 \mathrm{CH}) ; 5.27-5.25(\mathrm{~m}, 2 \mathrm{H}, 2 \mathrm{CH}) ; 5.20-5.19(\mathrm{~m}, 1 \mathrm{H}, \mathrm{CH})$; $4.92(\mathrm{~d}, 1 \mathrm{H}, \mathrm{J}=1,6 \mathrm{~Hz}, \mathrm{CH}) ; 4.71$ (dd, $2 \mathrm{H}, J=12,2 \mathrm{~Hz}, 53.1 \mathrm{~Hz}) ; 4.51-4.48(2 \mathrm{H}, J=5.2 \mathrm{~Hz}$, $\left.\mathrm{CH}_{2}\right) ; 4.29-4.23(\mathrm{~m}, 1 \mathrm{H}, \mathrm{CH}) ; 4.18-4.16\left(2 \mathrm{H}, \mathrm{J}=4.9 \mathrm{~Hz}, \mathrm{CH}_{2}\right) ; 4.08(\mathrm{~d}, 1 \mathrm{H}, J=2.4 \mathrm{~Hz}, \mathrm{CH})$; 4.05-4.02 (m, $1 \mathrm{H}, \mathrm{CH}) ; 3.86-3.82\left(\mathrm{~m}, 4 \mathrm{H}, 2 \mathrm{CH}_{2}\right)$; 3.71-3.69 (m, 2H, $\left.\mathrm{CH}_{2}\right)$; 3.64-3.61 (m, $2 \mathrm{H}$, $\left.\mathrm{CH}_{2}\right) ; 3.60-3.57\left(\mathrm{~m}, 4 \mathrm{H}, 2 \mathrm{CH}_{2}\right) ; 2.09\left(\mathrm{~s}, 3 \mathrm{H}, \mathrm{CH}_{3}\right) ; 2.07\left(3 \mathrm{H}, \mathrm{CH}_{3}\right) ; 1.98\left(\mathrm{~s}, 3 \mathrm{H}, \mathrm{CH}_{3}\right) ; 1.93$ (s, $\left.3 \mathrm{H}, \mathrm{CH}_{3}\right) .{ }^{13} \mathrm{C}$-NMR: (100 MHz, $\left.\mathrm{CDCl}_{3}\right): 170.90,170.86,170.17,170.01,169.86,161.33,152.80$, 147.18, 143.33, 130.55, 129.16, 124.84, 124.51, 122.67, 114.92, 96.88, 92.29, 77.43, 70.96, 70.67, 70.63, 70.62, 69.72, 68.78, 67.82, 66.43, 66.15, 66.15, 62.47, 61.71, 60.97, 50.45, 20.99, 20.91, 20.82, 20.79. ESI-MS (m/z): calculated for $\left[\mathrm{C}_{37} \mathrm{H}_{47} \mathrm{~N}_{5} \mathrm{O}_{14} \mathrm{H}\right]^{+}: 786.3198$ found: 786.0652. 
(E)-2-(2-(2-(4-(phenyldiazenyl)phenoxy)ethoxy)ethoxy)ethoxy)ethyl-triazoylmannopyranoside (13)

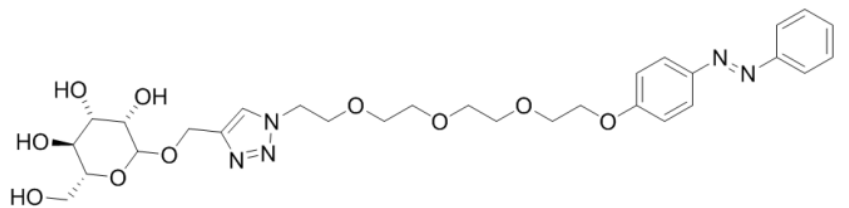

Compound (12) (90 mg, 0.11 $\mathrm{mmol}$ ) was dissolved in 10 $\mathrm{mL}$ of dry methanol followed by the addition of a catalytical amount of $\mathrm{NaOMe}(20 \mathrm{mg})$. After 30 minutes Amberlite ${ }^{\circledR}$ IR $120\left(\mathrm{H}^{+}\right)$ion exchange resin was added until a $\mathrm{pH}$ of 3 was reached. The resin was filtered off and the solution was evaporated to dryness. The title compound was obtained as an orange-brown hygroscopic solid. Yield: $61 \mathrm{mg}, 87 \% .{ }^{1} \mathrm{H}-\mathrm{NMR}$ (400 $\mathbf{M H z}, \mathrm{CD}_{3} \mathrm{OD}$ ): 8.22 (s, $\left.1 \mathrm{H}, \mathrm{CH}_{\text {triazole }}\right) ; 7.82-7.79(\mathrm{~m}, 4 \mathrm{H}, 4 \mathrm{CH}) ; 7.46-7.44(\mathrm{~m}, 2 \mathrm{H}, 2 \mathrm{CH}) ; 7.42-7.40(\mathrm{~m}, 1 \mathrm{H}, \mathrm{CH}) ; 7.02-$ $7.00(\mathrm{~d}, 2 \mathrm{H}, J=8.8 \mathrm{~Hz}, 2 \mathrm{CH}) ; 4.82(\mathrm{~s}, 1 \mathrm{H}, \mathrm{CH}) ; 4.71\left(\mathrm{dd}, 2 \mathrm{H}, \mathrm{J}=46,2 \mathrm{~Hz}, 12,7 \mathrm{~Hz}, \mathrm{CH}_{2}\right) ; 4.56$ $\left(\mathrm{t}, 2 \mathrm{H}, \mathrm{J}=5 \mathrm{~Hz}, \mathrm{CH}_{2}\right) ; 4.15\left(\mathrm{t}, 2 \mathrm{H}, \mathrm{J}=4.8 \mathrm{~Hz} ; \mathrm{CH}_{2}\right) ; 3.84\left(\mathrm{t}, 2 \mathrm{H}, \mathrm{J}=4.7 \mathrm{~Hz}, \mathrm{CH}_{2}\right) ; 3.81-3.79(\mathrm{~m}$, $2 \mathrm{H}, \mathrm{CH}_{2}$ ); 3.77 (bs, $\left.1 \mathrm{H}, \mathrm{CH}\right) ; 3.67-3.62\left(\mathrm{~m}, 4 \mathrm{H}, 1 \mathrm{CH}_{2}, 2 \mathrm{CH}\right) ; 3.59-3.55\left(\mathrm{~m}, 8 \mathrm{H}, 4 \mathrm{CH}_{2}\right) ; 3.50-$ 3.47 (m, 1H, CH). ${ }^{13} \mathrm{C}-\mathrm{NMR}:\left(100 \mathrm{MHz}, \mathrm{CD}_{3} \mathrm{OD}\right): 168.90,163.00,154.10,148.32,131.73$, 130.86, 130.27, 127.43, 127.16, 125.83, 123.58, 116.04, 101.26, 96.38, 75.20, 71.95, 71.80, 71.56, 71.53, 71.46, 70.74, 69.96, 69.07, 68.79, 68.58, 62.98, 60.12, 52.49. ESI-MS (m/z): calculated for $\left[\mathrm{C}_{29} \mathrm{H}_{39} \mathrm{~N}_{5} \mathrm{O}_{10} \mathrm{H}\right]^{+}: 617.27$ found 617.97

(E)-2-(2-(2-(4-(phenyldiazenyl)phenoxy)ethoxy)ethoxy)ethoxy)ethyl-triazoylperacetylgalactopyranoside (14)

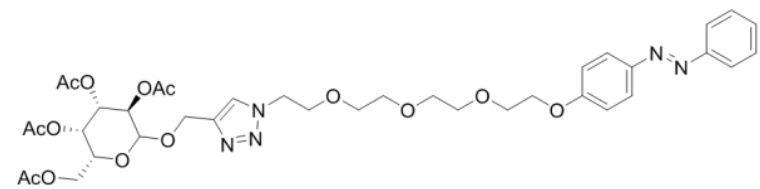

To a mixture of (11) $(50 \mathrm{mg})$ and (5) $(60 \mathrm{mg})$ in a degassed solvent mixture of $\mathrm{MeOH}$, DMF and water (1:2:1) was added sodium ascorbate (20 mg) and $\mathrm{CuSO}_{4}(10 \mathrm{mg})$. The suspension was stirred for $24 \mathrm{~h}$ under an atmosphere of argon followed by evaporation of all solvents. The residue was dissolved in $\mathrm{CHCl}_{3}(1 \mathrm{~mL})$ and subjected to silica column chromatography $\left(\mathrm{CHCl}_{3} / \mathrm{MeOH}\right.$, 9:1). The product was obtained as an orange oil. Yield: $88 \mathrm{mg}, 89 \%{ }^{1} \mathrm{H}-\mathrm{NMR}(400 \mathbf{M H z}$, $\mathrm{CDCl}_{3}$ ): 7.88-7.82 (m, 4H, 4CH); 7.72 (bs, $\left.1 \mathrm{H}, \mathrm{CH}_{\text {triazole }}\right) ; 7.46$ (t, J = 7.2 Hz, 2H, 2CH); 7.40 (t, $\mathrm{J}=7.2 \mathrm{~Hz}, 1 \mathrm{H}, \mathrm{CH}) ; 6.98(\mathrm{~d}, \mathrm{~J}=8.7 \mathrm{~Hz}, 2 \mathrm{H}, 2 \mathrm{CH}) ; 5.36(\mathrm{~d}, \mathrm{~J}=3.3 \mathrm{~Hz}, 1 \mathrm{H}, \mathrm{CH}) ; 5.18-5.16(\mathrm{~m}$, $1 \mathrm{H}, \mathrm{CH}) ; 4.99-4.97(\mathrm{~m}, 1 \mathrm{H}, \mathrm{CH}) ; 4.85\left(\mathrm{dd}, \mathrm{J}=12 \mathrm{~Hz}, 43 \mathrm{~Hz}, 2 \mathrm{H}, \mathrm{CH}_{2}\right) ; 4.63(\mathrm{~d}, \mathrm{~J}=6 \mathrm{~Hz}, 1 \mathrm{H}$, $\mathrm{CH}) ; 4.51-4.48\left(\mathrm{~m}, 2 \mathrm{H}, \mathrm{CH}_{2}\right) ; 4.18\left(\mathrm{t}, \mathrm{J}=4.3 \mathrm{~Hz}, 2 \mathrm{H}, \mathrm{CH}_{2}\right) ; 4.13-4.11\left(\mathrm{~m}, 2 \mathrm{H}, \mathrm{CH}_{2}\right) ; 3.91(\mathrm{t}, \mathrm{J}=$ $6.6 \mathrm{~Hz}, 1 \mathrm{H}, \mathrm{CH}) ; 3.86-3.83\left(\mathrm{~m}, 4 \mathrm{H}, 2 \mathrm{CH}_{2}\right) ; 3.70\left(\mathrm{t}, \mathrm{J}=4 \mathrm{~Hz}, 2 \mathrm{H}, \mathrm{CH}_{2}\right) ; 3.63-3.59(\mathrm{~m}, 6 \mathrm{H}, 3$ $\left.\mathrm{CH}_{2}\right) ; 2.10\left(\mathrm{~s}, 3 \mathrm{H}, \mathrm{CH}_{3}\right) ; 2.02\left(\mathrm{~s}, 3 \mathrm{H}, \mathrm{CH}_{3}\right) ; 1.94\left(\mathrm{~s}, 3 \mathrm{H}, \mathrm{CH}_{3}\right) ; 1.93\left(\mathrm{~s}, 3 \mathrm{H}, \mathrm{CH}_{3}\right) \cdot{ }^{13} \mathrm{C}-\mathrm{NMR}$ : (100 $\left.\mathrm{MHz}, \mathrm{CDCl}_{3}\right): 170.27,170.10,170.08,169.92,169.36,161.05,152.53,146.91,130.27,128.88$, 120 
$124.56,122.39,114.65,100.14,95.35,90.44,77.12,70.69,70.62,70.42,70.38,70.33,69.46$, 69.20, 68.59, 67.56, 66.90, 62.56, 61.10, 50.21, 20.57, 20.54, 20.50, 20.41. calculated for $\left[\mathrm{C}_{37} \mathrm{H}_{47} \mathrm{~N}_{5} \mathrm{O}_{14} \mathrm{H}\right]^{+}: 786.3198$ found: 786.0656 .

\section{(E)-2-(2-(2-(4-(phenyldiazenyl)phenoxy)ethoxy)ethoxy)ethoxy)ethyl-triazoyl-} galactopyranoside (15)

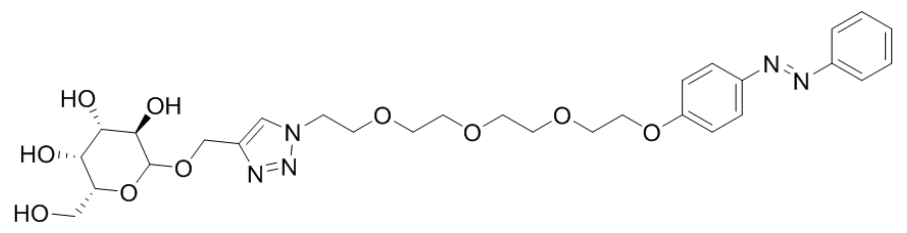

Compound (14) (90 mg, $0.11 \mathrm{mmol}$ ) was dissolved in $10 \mathrm{~mL}$ of dry methanol followed by the addition of a catalytic amount of NaOMe (20 mg). After 30 minutes Amberlite ${ }^{\circledR}$ IR $120\left(\mathrm{H}^{+}\right)$ion exchange resin was added until a $\mathrm{pH}$ of 3 was reached. The resin was filtered off and the solution was evaporated to dryness. The title compound was obtained as an orange-brown hygroscopic solid. Yield: $66 \mathrm{mg}, 97 \%{ }^{1} \mathrm{H}-\mathrm{NMR}$ (400 MHz, $\left.\mathrm{CD}_{3} \mathrm{OD}\right)$ : $8.07\left(\mathrm{~s}, 1 \mathrm{H}, \mathrm{CH}_{\text {triazole }}\right.$ ); 7.91-7.85 (m, 4H, $4 \mathrm{CH}) ; 7.54-7.52(\mathrm{~m}, 2 \mathrm{H}, 2 \mathrm{CH}) ; 7.48-7.46(\mathrm{~m}, 1 \mathrm{H}, \mathrm{CH}) ; 7.09(\mathrm{~d}, J=9.1 \mathrm{~Hz}, 2 \mathrm{H}, 2 \mathrm{CH}) ; 4.96(\mathrm{~d}$, $\left.J=12.4 \mathrm{~Hz}, 1 \mathrm{H}, \mathrm{CH}_{2}\right) ; 4.76\left(\mathrm{~d}, J=12.4 \mathrm{~Hz}, 2 \mathrm{H} \mathrm{CH}_{2}\right) ; 4.55\left(\mathrm{t}, \mathrm{J}=4.9 \mathrm{~Hz}, 2 \mathrm{H}, \mathrm{CH}_{2}\right) ; 4.33(\mathrm{~d}, J=$ $7.6 \mathrm{~Hz}, 1 \mathrm{H}, \mathrm{CH}) ; 4.24-4.22\left(\mathrm{~m}, 2 \mathrm{H}, \mathrm{CH}_{2}\right) ; 3.89-3.86\left(\mathrm{~m}, 4 \mathrm{H}, 2 \mathrm{CH}_{2}\right) ; 3.82-3.70\left(\mathrm{~m}, 4 \mathrm{H}, 2 \mathrm{CH}_{2}\right)$; 3.62-3.57 (m, 6H, $\left.3 \mathrm{CH}_{2}\right) ; 3.55-3.52\left(\mathrm{~m}, 2 \mathrm{H}, \mathrm{CH}_{2}\right) ; 3.48-3.44(\mathrm{~m}, 1 \mathrm{H}, \mathrm{CH}) .{ }^{13} \mathrm{C}-\mathrm{NMR}:(100 \mathrm{MHz}$, $\left.\mathrm{CD}_{3} \mathrm{OD}\right): 163.43,154.13,148.60,131.66,130.23,128.41,126.15,125.78,123.54,116.05$, 104.27, 76.84, 74.94, 71.82, 71.59, 71.50, 70.76, 70.38, 70.35, 69.07, 63.01, 62.62, 51.47. ESI-MS (m/z): calculated for $\left[\mathrm{C}_{29} \mathrm{H}_{39} \mathrm{~N}_{5} \mathrm{O}_{10} \mathrm{H}\right]^{+}: 617.27$ found: 617.93 .

\section{Rhodamine-azobenzene conjugate (16)}

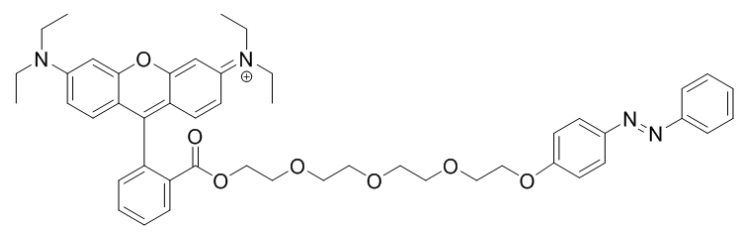

(2) (300 $\mathrm{mg}, 0,8 \mathrm{mmol}$ ) was dissolved in $20 \mathrm{~mL}$ of dichloromethane followed by the addition of HOBt (110 $\mathrm{mg}, 0,8$ $\mathrm{mmol})$ and $\operatorname{EDCl}(0,13 \mathrm{~mL}, 0,8$ mmol). After 20 minutes of stirring rhodamine B $(380 \mathrm{mg}, 0.8 \mathrm{mmol})$ was added and stirring was continued for $18 \mathrm{~h}$. After that time the solvent was removed in vacuo and the residue was subjected to silica gel column chromatography. (DCM/EtOH - 9:1, Rf = 0,88), Yield: $58 \mathrm{mg}, 9 \%$. 'H-NMR (400 $\mathrm{MHz}^{\mathrm{C}} \mathrm{CDCl}_{3}$ ): 8.30 (dd, J = 7.0 Hz, $0.7 \mathrm{~Hz}, 1 \mathrm{H}, \mathrm{CH}$ ); 7.91-7.83 (m, 4H, 4CH); $7.78(\mathrm{dt}, \mathrm{J}=6.5 \mathrm{~Hz}, 0.8 \mathrm{~Hz}, 1 \mathrm{H}, \mathrm{CH}) ; 7.69(\mathrm{dt}, \mathrm{J}=7.0 \mathrm{~Hz}, 0.8 \mathrm{~Hz}$, $1 \mathrm{H}, \mathrm{CH}) ; 7.50-7.39(\mathrm{~m}, 3 \mathrm{H}, 3 \mathrm{CH}) ; 7.29(\mathrm{~d}, \mathrm{~J}=7.5 \mathrm{~Hz}, 1 \mathrm{H}, \mathrm{CH}) ; 7.04-6.96(\mathrm{~m}, 4 \mathrm{H}, 4 \mathrm{CH}) ; 6.82-$ $6.80(\mathrm{~m}, 4 \mathrm{H}, 4 \mathrm{CH}) ; 4.22-4.16\left(\mathrm{~m}, 2 \mathrm{H}, \mathrm{CH}_{2}\right) ; 3.89-3.84\left(\mathrm{~m}, 2 \mathrm{H}, \mathrm{CH}_{2}\right) ; 3.75-3.52(\mathrm{~m}, 2 \mathrm{OH}, 10$ 
$\left.\mathrm{CH}_{2}\right)$; $1.27\left(\mathrm{t}, \mathrm{J}=7.0 \mathrm{~Hz}, 12 \mathrm{H}, 4 \mathrm{CH}_{3}\right) .{ }^{13} \mathrm{C}-\mathrm{NMR}:\left(100 \mathrm{MHz}, \mathrm{CDCl}_{3}\right): 165.66,161.91,159.52$, 158.47, 156.19, 153.43, 153.38, 147.79, 147.74, 134.39, 133.86, 132.17, 131.98, 131.16, 131.08, 131.04, 130.99, 130.40, 129.75, 129.72, 127.50, 125.41, 123.25, 116.86, 115.57, 115.53, 115.49, 114.79, 114.26, 97.11, 77.83, 73.23, 71.53, 71.52, 71.34, 71.26, 71.22, 71.17, 70.98, 70.31, 69.39, 68.42, 68.38, 46.77, 30.39, 13.32. ESI-MS: calculated for $\left[\mathrm{C}_{48} \mathrm{H}_{55} \mathrm{~N}_{4} \mathrm{O}_{7}\right]^{+}: 799.4065$, found 799.5651.

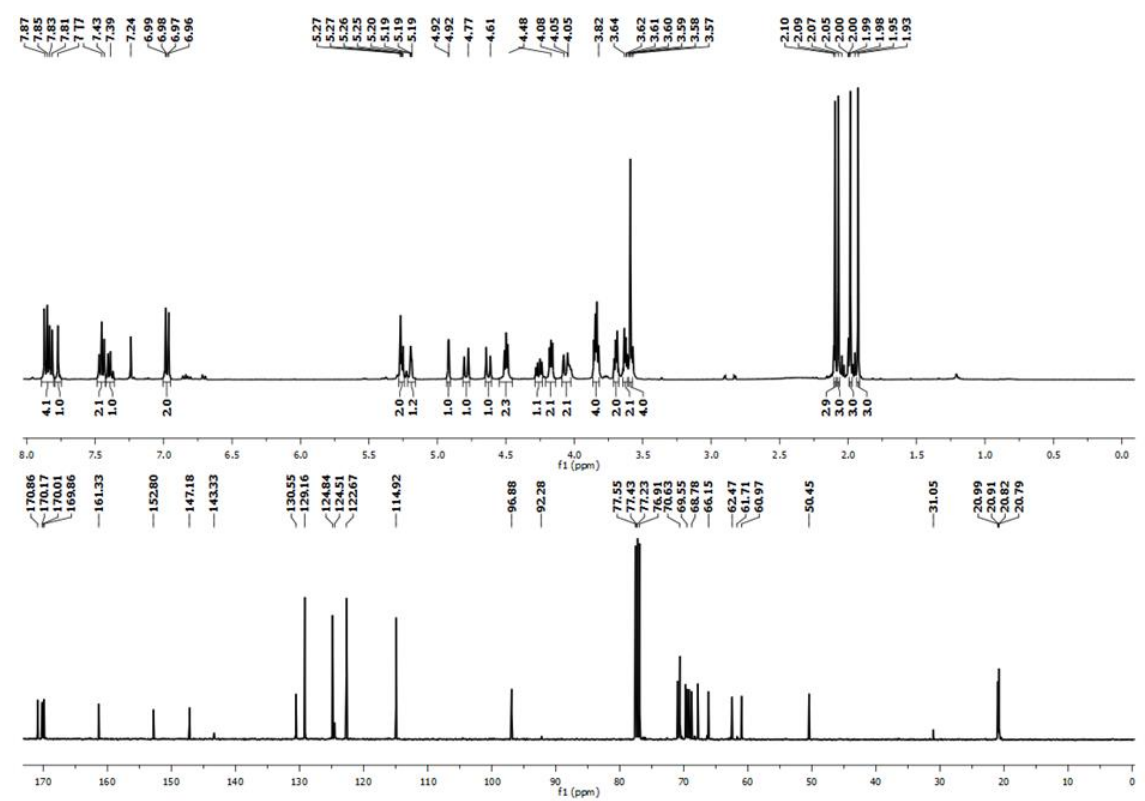

Figure 5.7. ${ }^{1} \mathrm{H}-\mathrm{NMR}$ and ${ }^{13} \mathrm{C}-\mathrm{NMR}$ of compound (12) in $\mathrm{CDCl}_{3}$ 


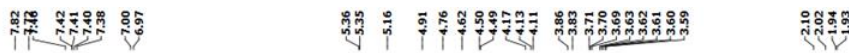

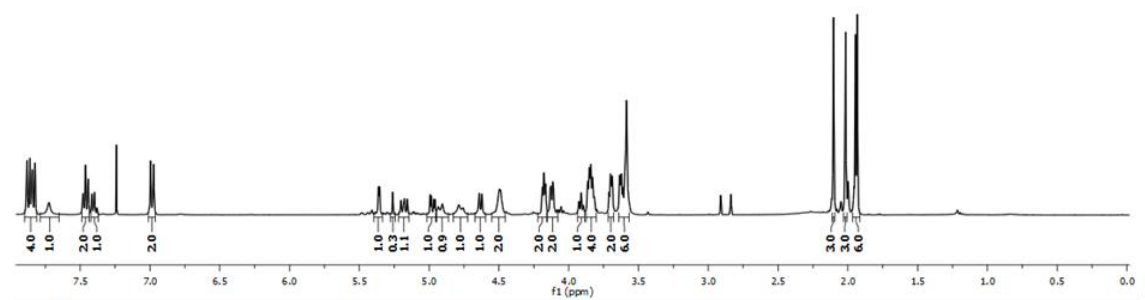

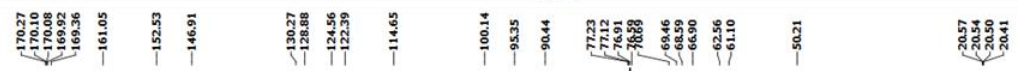

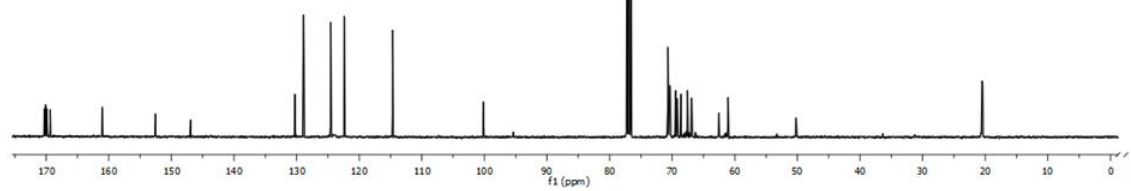

Figure 5.8. ${ }^{1} \mathrm{H}-\mathrm{NMR}$ and ${ }^{13} \mathrm{C}-\mathrm{NMR}$ of compound (14) in $\mathrm{CDCl}_{3}$.

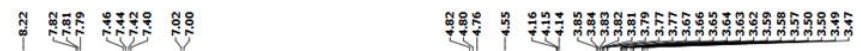

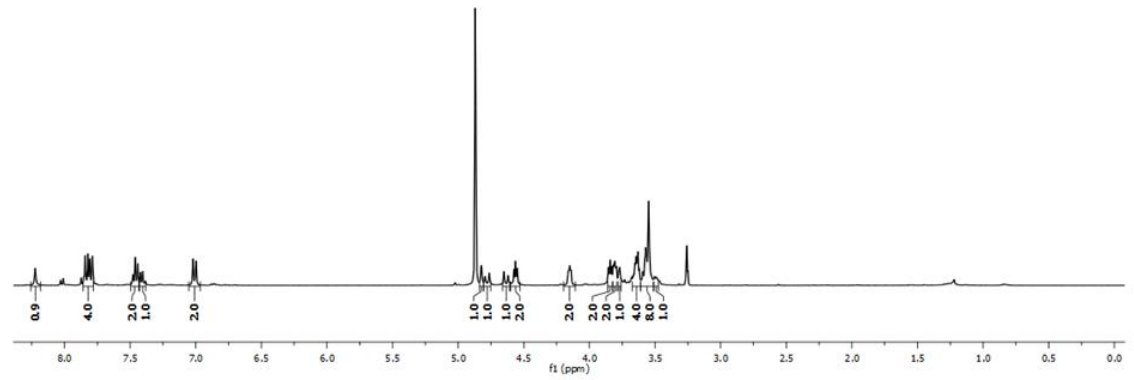

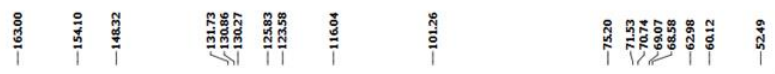
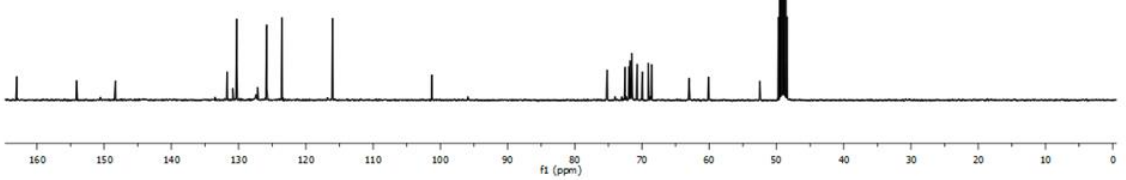

Figure 5.9. ${ }^{1} \mathrm{H}-\mathrm{NMR}$ and ${ }^{13} \mathrm{C}-\mathrm{NMR}$ of compound (13) in $\mathrm{CDCl}_{3}$ 


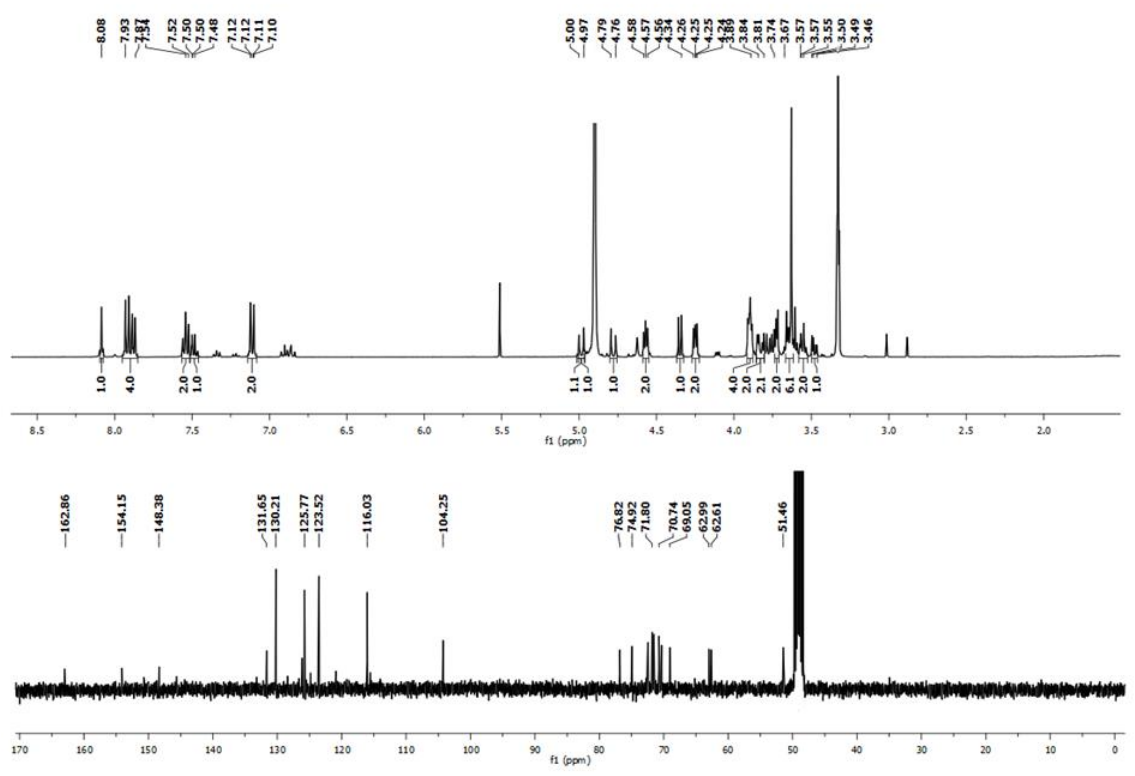

Figure 5.10. ${ }^{1} \mathrm{H}-\mathrm{NMR}$ and ${ }^{13} \mathrm{C}-\mathrm{NMR}$ of compound (15) in $\mathrm{CDCl}_{3}$.

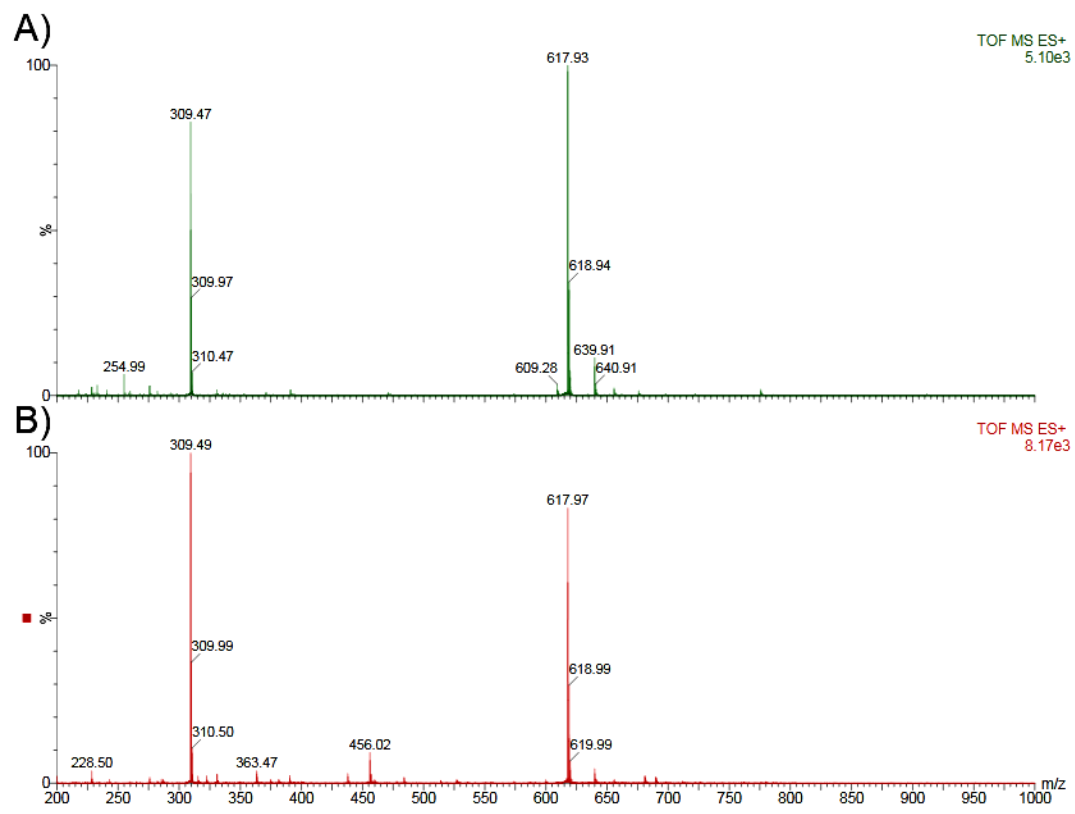

Figure 5.11. ESI-TOF-MS of A) compound (15) and B) compound (13) 


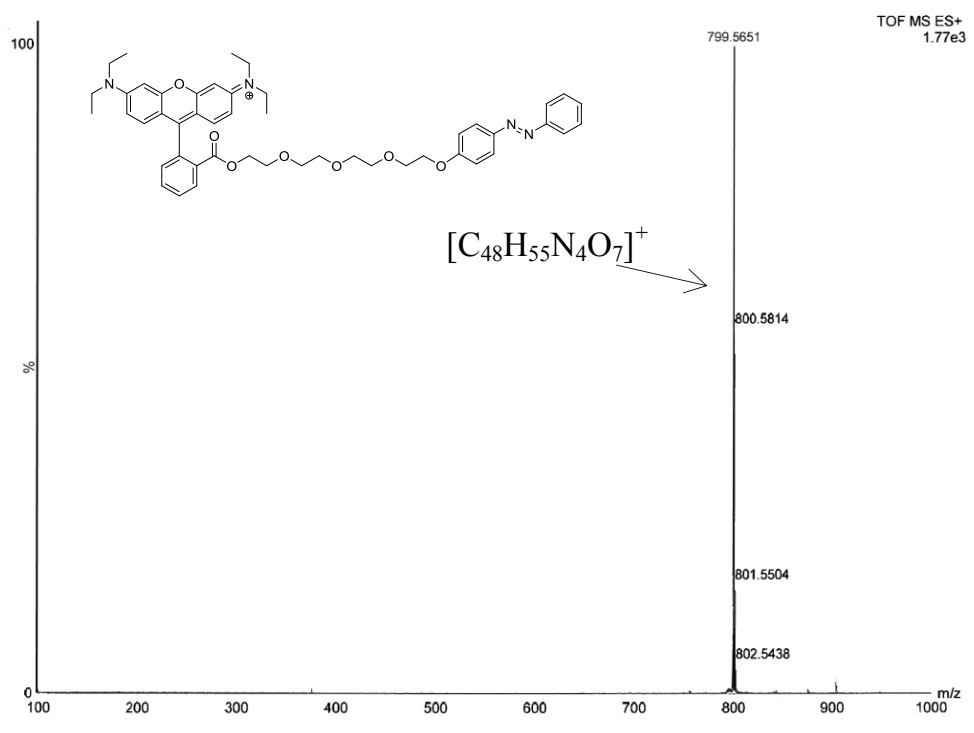

Figure 5.12. TOF MS-ES+ spectrum of compound (16)

Monolayers on gold substrates for QCM. Standard gold coated QCM sensors from Qsense (QSX301) were cleaned by immersing them in piranha (1:3 mixture of concentrated $\mathrm{H}_{2} \mathrm{SO}_{4}$ and $30 \% \mathrm{H}_{2} \mathrm{O}_{2}$ ) for $15 \mathrm{~s}$. (Warning: piranha should be handled with caution; it can detonate unexpectedly). After thorough rinsing with MilliQ water, they were placed in absolute ethanol for $10 \mathrm{~min}$. The substrates were subsequently placed in a freshly prepared $0.1 \mathrm{mM}$ solution of $\beta-\mathrm{CD}$ heptathioether in $\mathrm{EtOH}$ and $\mathrm{CHCl}_{3}(1: 2 \mathrm{v} / \mathrm{v})$ for $16 \mathrm{~h}$ at $60^{\circ} \mathrm{C}$. The substrates were then rinsed with $\mathrm{CHCl}_{3}, \mathrm{EtOH}$ and MilliQ water.

Monolayers on glass substrates for microcontact printing. The glass substrates were cleaned and activated by immersing in piranha for 30 mins followed by washing with water, ethanol and thorough drying. The surface was then functionalized with $\mathrm{N}-\left[3^{-}\right.$ (trimethoxysilyl)propyl]ethylenediamine (TPEDA) by overnight chemical vapor deposition using a vacuum desiccator. The substrates were then thoroughly cleaned using ethanol and toluene after which it was incubated in a $1 \mathrm{mM}$ solution of 1,4phenylene di-isothiocyanate (ITC) in dry toluene for $2 \mathrm{hr}$ at $50^{\circ} \mathrm{C}$. The substrates were then washed thoroughly with toluene, ethanol and water and finally incubated with a $1 \mathrm{mM}$ solution of per-6-amino- $\beta$-cyclodextrin in MilliQ water for $2 \mathrm{hr}$ at $50^{\circ} \mathrm{C}$. The substrates were finally washed thoroughly with water, dried and stored in $\mathrm{N}_{2}$ atmosphere until use. 
QCM experiments. The QCM experiments were conducted using the Q-Sense E1 instrument with the standard Flow Module. All binding studies were conducted with a flow rate of $100 \mu \mathrm{L} / \mathrm{min}$ at a temperature of $22{ }^{\circ} \mathrm{C}$. Resonance frequency and dissipation values were determined automatically by the instrument and only the changes in these values were presented as the response. Binding affinity experiments of Azo-Man onto $\beta-C D$ monolayers were done using water as the solvent. Binding experiments involving ConA and bacteria were done in a $10 \mathrm{mM}$ HEPES, $137 \mathrm{mM} \mathrm{NaCl}, \mathrm{pH} 7$ buffer containing $1 \mathrm{mM} \mathrm{MnCl} 2$ and $1 \mathrm{mM} \mathrm{CaCl}$.

Microcontact printing. Poly(dimethylsiloxane) (PDMS) stamps were prepared by casting a 10:1 (v/v) mixture of Sylgard 184 elastomer and curing agent (Dow Corning) against a patterned silicon master. After curing the stamps at $60^{\circ} \mathrm{C}$ overnight, they were peeled off from the master before using. The individually cut out stamps were inked with the solution containing the azobenzene compounds for 10 mins after which they were dried with a stream of $\mathrm{N}_{2}$ gas. These were then stamped on the substrate for 10 mins with $15 \mathrm{~g}$ weight on top. The substrate was then briefly washed with water and dried. Backfilling was done by incubating with a $1 \mathrm{mM}$ Azo-PEG5000 solutions for 5 mins. Finally, the protein or bacteria were immobilized by incubating them on the substrate for 10 mins.

Bacterial immobilization. The bacterial strains ORN178 and ORN208 (kind gift from Prof. Luc Brunsveld, TU/e) were grown overnight in LB media using tetracycline as the selective antibiotic. These were then spun down at $5000 \mathrm{~g}$ for 10 mins and the supernatant was discarded. The bacteria were washed twice with $10 \mathrm{mM}$ HEPES, 137 $\mathrm{mM} \mathrm{NaCl}, \mathrm{pH} 7$ buffer by centrifugation and resuspension. Finally the bacteria were reconstituted in this buffer and their optical density at $600 \mathrm{~nm}$ was measured. For QCM and microcontact printing, the bacterial solutions were then diluted down to an O.D. of 0.1 and $\mathrm{MnCl}_{2}, \mathrm{CaCl}_{2}$ were added to final concentration of $1 \mathrm{mM}$ each.

Fluorescence studies. An Olympus microscope IX71 with appropriate filters were used for recording fluorescent images.

ConA was conjugated with fluorescein using 5-carboxyfluorescein, succinimidyl ester (5-FAM) from Molecular Probes Europe. 5-FAM was added to a $1 \mathrm{mg} / \mathrm{mL}$ solution of ConA in $\mathrm{pH} 9$ carbonate buffer to obtain a final dye concentration of $200 \mu \mathrm{M}$. This was incubated at room temperature for 60 mins with occasional shaking. The dyeconjugated protein was then separated from unreacted dye and rebuffered into the above mentioned buffer using Zeba desalting spin column with a cutoff of $7 \mathrm{kDa}$. ConA was conjugated with $\mathrm{Cy} 3$ dye from GE Healthcare using a similar protocol. 
Surface immobilized bacteria were stained with a $1 \mu \mathrm{g} / \mathrm{mL}$ Hoechst (H33342) solution for 5 mins after which they were washed, dried and viewed under the microscope.

UV irradiation was performed using a Spectroline miniature ultraviolet pencil lamp (model 36-380) having an average intensity of $1000 \mu \mathrm{W} / \mathrm{cm}^{2}$ of $365 \mathrm{~nm}$ radiation at 1 in. The lamp was always placed at approximately $3 \mathrm{~cm}$ away from the sample in a dark box. Subsequent washing and drying of the substrates was always carried out in the dark. UV irradiation of all dry patterned substrates, irrespective of what dye was used, did not diminish the fluorescence intensity, confirming that photo-bleaching of the dye did not occur.

\subsection{References}

(1) Brinkmann, J., Cavatorta, E., Sankaran, S., Schmidt, B., van Weerd, J., and Jonkheijm, P. (2014) About supramolecular systems for dynamically probing cells. Chem. Soc. Rev. 43, 4449-4469.

(2) Robertus, J., Browne, W. R., and Feringa, B. L. (2010) Dynamic control over cell adhesive properties using molecular-based surface engineering strategies. Chem. Soc. Rev. 39, 354-378.

(3) Mendes, P. M. (2008) Stimuli-responsive surfaces for bio-applications. Chem. Soc. Rev. 37, 2512-2529.

(4) Voskuhl, J., Brinkmann, J., and Jonkheijm, P. (2014) Advances in contact printing technologies of carbohydrate, peptide and protein arrays. Arrays 18, 1-7.

(5) Petkau-Milroy, K., Sonntag, M. H., and Brunsveld, L. (2013) Modular columnar supramolecular polymers as scaffolds for biomedical applications. Chem. - Eur. J. 19, 10786-10793.

(6) Neirynck, P., Brinkmann, J., An, Q., van der Schaft, D. W. J., Milroy, L.-G., Jonkheijm, P., and Brunsveld, L. (2013) Supramolecular control of cell adhesion via ferrocene-cucurbit[7]uril hostguest binding on gold surfaces. Chem. Commun. 49, 3679-3681.

(7) An, Q., Brinkmann, J., Huskens, J., Krabbenborg, S., de Boer, J., and Jonkheijm, P. (2012) A supramolecular system for the electrochemically controlled release of cells. Angew. Chem. Int. Ed. 51, $12233-12237$.

(8) Tian, F., Cziferszky, M., Jiao, D., Wahlström, K., Geng, J., and Scherman, O. A. (2011) Peptide separation through a $\mathrm{CB}[8]$-mediated supramolecular trap-and-release process. Langmuir 27 , 1387-1390.

(9) Yang, L., Gomez-Casado, A., Young, J. F., Nguyen, H. D., Cabanas-Danés, J., Huskens, J., Brunsveld, L., and Jonkheijm, P. (2012) Reversible and oriented immobilization of ferrocenemodified proteins. J. Am. Chem. Soc. 134, 19199-19206.

(10) Thakar, D., Coche-Guérente, L., Claron, M., Wenk, C. H. F., Dejeu, J., Dumy, P., Labbé, P., and Boturyn, D. (2014) Redox-driven host-guest interactions allow the controlled release of captured cells on RGD-functionalized surfaces. ChemBioChem 15, 377-381.

(11) Onclin, S., Huskens, J., Ravoo, B. J., and Reinhoudt, D. N. (2005) Molecular boxes on a molecular printboard: encapsulation of anionic dyes in immobilized dendrimers. Small 1, 852-857.

(12) Perl, A., Gomez-Casado, A., Thompson, D., Dam, H. H., Jonkheijm, P., Reinhoudt, D. N., and Huskens, J. (2011) Gradient-driven motion of multivalent ligand molecules along a surface functionalized with multiple receptors. Nat Chem 3, 317-322.

(13) Campiña, J. M., Martins, A., and Silva, F. (2009) Immobilization of $\beta$-cyclodextrin on gold surfaces by chemical derivatization of an 11-amino-1-undecanthiol self-assembled monolayer. Electrochimica Acta 55, 90-103. 
(14) Nijhuis, C. A., Yu, F., Knoll, W., Huskens, J., and Reinhoudt, D. N. (2005) Multivalent dendrimers at molecular printboards: influence of dendrimer structure on binding strength and stoichiometry and their electrochemically induced desorption. Langmuir 21, 7866-7876.

(15) An, Q., Li, G., Tao, C., Li, Y., Wu, Y., and Zhang, W. (2008) A general and efficient method to form self-assembled cucurbit[n]uril monolayers on gold surfaces. Chem. Commun. 1989-1991.

(16) Young, J. F., Nguyen, H. D., Yang, L., Huskens, J., Jonkheijm, P., and Brunsveld, L. (2010) Strong and reversible monovalent supramolecular protein immobilization. ChemBioChem 11, 180183.

(17) Wasserberg, D., Uhlenheuer, D. A., Neirynck, P., Cabanas-Danés, J., Schenkel, J. H., Ravoo, B. J., An, Q., Huskens, J., Milroy, L.-G., and Brunsveld, L. (2013) Immobilization of ferrocene-modified SNAP-fusion proteins. Int. J. Mol. Sci. 14, 4066-4080.

(18) Shi, X.-J., Chen, G.-J., Wang, Y.-W., Yuan, L., Zhang, Q., Haddleton, D. M., and Chen, H. (2013) Control the wettability of poly(N-isopropylacrylamide-co-1-adamantan-1-ylmethyl acrylate) modified surfaces: the more Ada, the bigger impact? Langmuir 29, 14188-14195.

(19) Tian, F., Cheng, N., Nouvel, N., Geng, J., and Scherman, O. A. (2010) Site-selective immobilization of colloids on au substrates via a noncovalent supramolecular "handcuff." Langmuir 26, 5323-5328.

(20) Roling, O., Mardyukov, A., Krings, J. A., Studer, A., and Ravoo, B. J. (2014) Polymer brushes exhibiting versatile supramolecular interactions grown by nitroxide-mediated polymerization and structured via microcontact chemistry. Macromolecules 47, 2411-2419.

(21) González-Campo, A., Brasch, M., Uhlenheuer, D. A., Gómez-Casado, A., Yang, L., Brunsveld, L., Huskens, J., and Jonkheijm, P. (2012) Supramolecularly oriented immobilization of proteins using cucurbit[8]uril. Langmuir 28, 16364-16371.

(22) Gong, Y.-H., Li, C., Yang, J., Wang, H.-Y., Zhuo, R.-X., and Zhang, X.-Z. (2011) Photoresponsive "smart template" via host-guest interaction for reversible cell adhesion. Macromolecules 44, 7499-7502.

(23) Tian, F., Jiao, D., Biedermann, F., and Scherman, O. A. (2012) Orthogonal switching of a single supramolecular complex. Nat Commun 3, 1207.

(24) Bortolus, P., and Monti, S. (1987) cis-trans photoisomerization of azobenzene-cyclodextrin inclusion complexes. J. Phys. Chem. 91, 5046-5050.

(25) Fenske, T., Korth, H.-G., Mohr, A., and Schmuck, C. (2012) Advances in switchable supramolecular nanoassemblies. Chem. - Eur. J. 18, 738-755.

(26) Stoffelen, C., Voskuhl, J., Jonkheijm, P., and Huskens, J. (2014) Dual stimuli-responsive selfassembled supramolecular nanoparticles. Angew. Chem. Int. Ed. 53, 3400-3404.

(27) Lan, Y., Wu, Y., Karas, A., and Scherman, O. A. (2014) Photoresponsive hybrid raspberry-like colloids based on cucurbit[8]uril host-guest interactions. Angew. Chem. Int. Ed. 53, 2166-2169.

(28) del Barrio, J., Horton, P. N., Lairez, D., Lloyd, G. O., Toprakcioglu, C., and Scherman, O. A. (2013) Photocontrol over cucurbit[8]uril complexes: stoichiometry and supramolecular polymers. J. Am. Chem. Soc. 135, 11760-11763.

(29) Nalluri, S. K. M., and Ravoo, B. J. (2010) Light-responsive molecular recognition and adhesion of vesicles. Angew. Chem. Int. Ed. 49, 5371-5374.

(30) Nalluri, S. K. M., Voskuhl, J., Bultema, J. B., Boekema, E. J., and Ravoo, B. J. (2011) Lightresponsive capture and release of DNA in a ternary supramolecular complex. Angew. Chem. Int. Ed. 50, 9747-9751.

(31) Samanta, A., Stuart, M. C. A., and Ravoo, B. J. (2012) Photoresponsive capture and release of lectins in multilamellar complexes. J. Am. Chem. Soc. 134, 19909-19914. 
(32) Wan, P., Jiang, Y., Wang, Y., Wang, Z., and Zhang, X. (2008) Tuning surface wettability through photocontrolled reversible molecular shuttle. Chem. Commun. 5710-5712.

(33) Jiang, Y., Wan, P., Smet, M., Wang, Z., and Zhang, X. (2008) Self-assembled monolayers of a malachite green derivative: surfaces with $\mathrm{pH}$ - and UV-responsive wetting properties. Adv. Mater. 20, 1972-1977.

(34) Yamaguchi, H., Kobayashi, Y., Kobayashi, R., Takashima, Y., Hashidzume, A., and Harada, A. (2012) Photoswitchable gel assembly based on molecular recognition. Nat Commun 3, 603.

(35) Kaufman, R. J., and Sidhu, R. S. (1982) Synthesis of aryl cluster glycosides by cyclotrimerization of 2-propynyl carbohydrate derivatives. J. Org. Chem. 47, 4941-4947.

(36) Lankshear, M. D., Dudley, I. M., Chan, K.-M., Cowley, A. R., Santos, S. M., Felix, V., and Beer, P. D. (2008) Cooperative and ion-pair recognition by heteroditopic calix[4]diquinone receptors. Chem. - Eur. J. 14, 2248-2263.

\subsection{Supporting information}

\subsubsection{Photoisomerization}

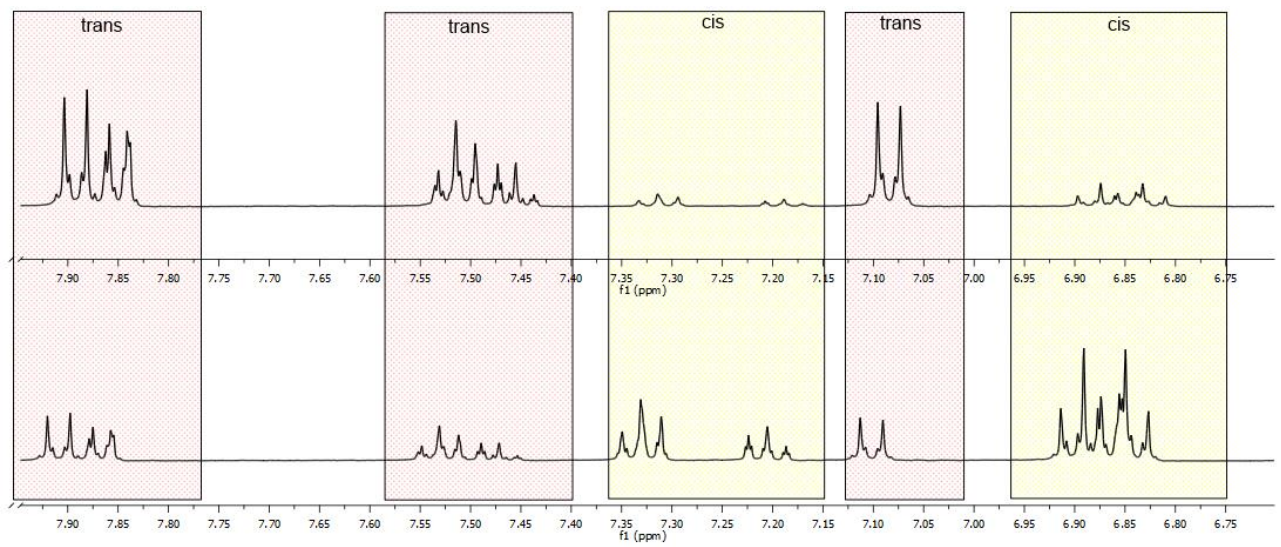

Figure S5.1: ${ }^{1} \mathrm{H}-\mathrm{NMR}$ of the aromatic region of compound (15) before and after irradiation for 10 minutes with a common UV-lamp $(365 \mathrm{~nm})$. 


\subsubsection{Release of surface immobilized ConA using UV irradiation}

Without UV

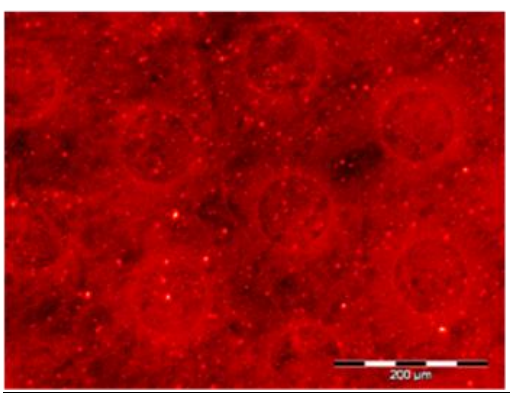

With UV

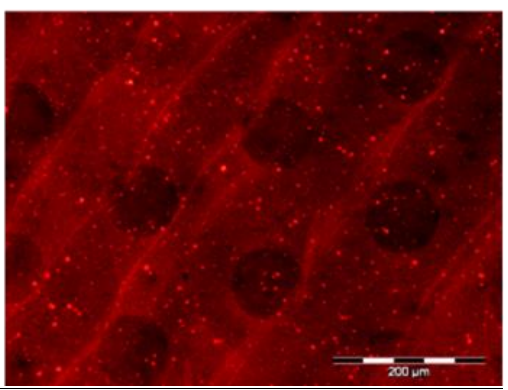

Figure 55.2. Patterns of Azo-Man was made on B-CD glass monolayers and backfilled with Azo$\mathrm{PEG}_{5000}$ as explained in the main text. This was then incubated with ConA-Cy3 for 10 mins and incubated in MilliQ water for 10 mins. One substrate was kept in the dark and the other was exposed to UV irradiation during this time. The substrates were then washed and dried thoroughly. Fluorescence images showed red dot patterns for the substrate without UV irradiation and dark dots for the surface that was exposed to UV. The incubation in water and washing steps however caused a diffusion of ConA-Cy3 over the rest of the surface causing higher background fluorescence. 


\section{Chapter 6}

\section{Photo-responsive cucurbit[8]uril-mediated adhesion of bacteria on supported lipid bilayers}

In this work the development of a photo-responsive platform for the presentation of bioactive ligands to study receptor-ligand interactions has been described. For this purpose, supramolecular host-guest chemistry and supported lipid bilayers (SLBs) have been combined in a microfluidic device. Quartz crystal microbalance with dissipation monitoring ( $Q C M-D)$ studies on methyl viologen $\left(M^{2+}\right)$-functionalized oligo ethylene glycol-based self-assembled monolayers, gel and liquid-state SLBs have been compared for their non-fouling properties in the case of ConA. In combination with bacterial adhesion test, negligible non-specific bacterial adhesion was observed only in the case of methyl viologen modified liquid-state SLBs. Therefore, liquid state SLBs have been identified as most suitable for studying specific cell interactions when methyl viologen is incorporated as a guest on the surface. The photo-switchable supramolecular ternary complex is formed by assembling cucurbit[8]uril ( $\mathrm{CB}[8])$ and an azobenzene-mannose conjugate (Azo-Man) onto $\mathrm{MV}^{2+}$-functionalized liquid-state SLBs and the assembly process has been characterized using QCM-D and fluorescence techniques. Mannose has been found to enable binding of E. coli via cell-surface receptors on the non-fouling supramolecular SLBS. Optical switching of the azobenzene moiety allowed us to 'erase' the bioactive surface after bacterial binding, providing the potential to develop reusable sensors. Localized photo-release of bacterial cells has also been shown indicating the possibility of optically guiding cellular growth, migration and inter-cellular interactions.

This work in this chapter was done in collaboration with Dr. Jasper van Weerd and Dr. Jens Voskuhl and is part of the article: Sankaran, S., ${ }^{\#}$ van Weerd, J., " Voskuhl, J., Karperien, M., and Jonkheijm, P. (2015) Photo-responsive cucurbit[8]uril-mediated adhesion of bacteria on supported lipid bilayers. Small. doi: 10.1002/smll.201502471

\# Equal Authorship 


\subsection{Introduction}

Surface immobilization strategies of bioactive ligands like peptides, carbohydrates, DNA and proteins have enabled widespread development of functional surfaces for biomedical applications and studies. ${ }^{1-4}$ Preliminary work in the field dealt with identifying biologically non-fouling components that could be deposited on surfaces and also chemically modified for the presentation of bioactive ligands. Some of the most successful strategies include self-assembled monolayers (SAMs), ${ }^{5,6}$ polymeric networks, ${ }^{7,8}$ hydrogels ${ }^{9}$ and supported lipid bilayers (SLBs). ${ }^{10}$ Such surfaces have been used to develop biosensors for detection and diagnostics, ${ }^{11,12}$ medical implant coatings, ${ }^{13}$ platforms for studying cellular processes ${ }^{14}$ and tissue engineering. ${ }^{15}$ However living entities predominantly exist in, interact with and modify a constantly changing environment. This led to the requirement for bioactive ligands to be presented in a highly dynamic manner to mimic the natural environment of eukaryotic and prokaryotic cells. Consequently, strategies are being developed to not only introduce such dynamic properties but also responsiveness, which is the ability to change some properties of the system in response to an external stimulus. Chemical, optical and electrochemical stimuli-responsive systems enable us to study biological processes that are triggered by a change in the environment. ${ }^{16,17}$ While traditional surface immobilization strategies allow for spatial control of bioactive ligands, ${ }^{18}$ dynamics and responsiveness allow for temporal control of these ligands. ${ }^{19,20}$

In this light, supported lipid bilayers have emerged as a powerful system for the development of a biomimetic surface since they are representative of the cell membrane. They can be used as model surfaces to study cell-ECM and cell-cell interactions. ${ }^{21-24}$ Supramolecular chemistry has been successfully used in generation of several dynamic and responsive architectures by virtue of the reversible non-covalent forces involved in the self-assembly process. ${ }^{25}$ By careful design and selection of the molecular components and the environment in which they interact, materials with novel properties can be developed. Host-guest systems have been especially successful towards the development of novel architectures for biomedical applications. ${ }^{26}$ These systems have the advantage that they operate in an aqueous environment with micromolar affinities and with reasonable specificities. A few surface confined molecular platforms have been developed using host molecules such as cyclodextrins and cucurbiturils for addressing living cells. Usually the host or guest molecule is anchored to the surface while its counterpart is conjugated to a bioactive ligand, allowing for dynamic and reversible presentation of ligands. Moreover, responsive 
presentation of ligands is made possible by competition with high affinity guests or a change in molecular properties such as, photo-isomerization of azobenzenes, ${ }^{19,27}$ and redox cycling of methylviologen and ferrocene..$^{20,28}$ Modifications caused by such stimuli can change the guest molecule's affinity towards the host, causing it to either bind stronger or get expelled. A few self-assembled monolayer based platforms have been recently developed that were able to address and manipulate cells. Preliminary reports of electro- and photo-responsive supramolecular surfaces for mammalian and bacterial cells have emerged in the last few years. ${ }^{20,27-29}$ These reports highlight the promise of employing supramolecular chemistry to develop dynamic and responsive platforms to address living entities. However, further exploration and careful characterization of the surface assembly process is required to understand and improve various aspects of these systems, such as their non-fouling nature, cell adhesion effects and localized responsiveness. In this chapter, we attempt to address these issues by combining the versatility of supramolecular chemistry with the biomimetic nature of SLBs.

For the first time, we report the development of a supramolecular host-guest system incorporated on SLBs enabling the photo-responsive display of a bioactive ligand for the selective adhesion and release of living cells. To this end, bacterial adhesion to mannose was explored for several reasons. Uropathogenic E. coli initiates an infection by binding to mannose sugar molecules found in glycoproteins and glycolipids of the glycocalyx of target cells. This binding event is mediated by the FimH receptors on the bacterial Type1 pili and exhibits complex relations to the surface density of mannose and shear stress induced by the flow of bodily fluids. ${ }^{30}$ Several strategies have been devised to study pathogen binding and develop suitable inhibitory molecules. ${ }^{31-33}$ Still, novel platforms are required to understand pathogen binding and allow for the rapid detection of these pathogens in medical samples. Bacteria pose additional challenges in biosensing due to their small size, highly heterogeneous surface components, motility and negatively charged cell surface causing non-specific electrostatic interactions with surfaces. We carefully developed our platform to systematically address these issues.

As shown in Figure 6.1, we used SLBs as the biomimetic background layer onto which we were able to assemble a ternary host-guest complex. First, the SLB was chemically modified with a thiolated methylviologen $\left(\mathrm{MV}^{2+}-\mathrm{SH}\right)$. Secondly, methylviologen $\left(\mathrm{MV}^{2+}\right)$ acts as the first guest molecule that can bind within the cavity of cucurbit[8]uril $(C B[8])$, the supramolecular host molecule. $C B[8]$ is a hollow pumpkin-shaped macrocylic host molecule made of 8 glycouril monomer units. ${ }^{34}$ The cavity is 
hydrophobic and can accommodate aromatic guest molecules. The rims of the cavity consist of polar carbonyl groups, allowing for ion-dipole interactions with positively charged molecules such as $\mathrm{MV}^{2+}$. The cavity is also big enough to accommodate a second guest molecule such as azobenzene. ${ }^{35}$ Azobenzene, in its trans form can form a stable ternary complex with $\mathrm{CB}[8]$ and $\mathrm{MV}^{2+}$. Thirdly, for specific adhesion of $\mathrm{E}$. coli, we conjugated the azobenzene to a mannose unit (Azo-Man) with a triethyleneglycol linker. Lastly, upon irradiation with $360 \mathrm{~nm}$ UV light, azobenzene photo-isomerizes into the cis form. The cis form is sterically hindered to fit within the cavity of $\mathrm{CB}[8]$, causing the affinity to be drastically reduced and as a result azobenzene is expelled from the cavity. In this study, mannose-modified azobenzene was used to impart photoresponsiveness to the system. Here, bacterial cells can normally bind to the surfaceanchored mannose units but on application of $360 \mathrm{~nm}$ UV light, the expulsion of cis Azo-Man from the $\mathrm{CB}[8]$ cavity causes the removal of the cell from the surface.

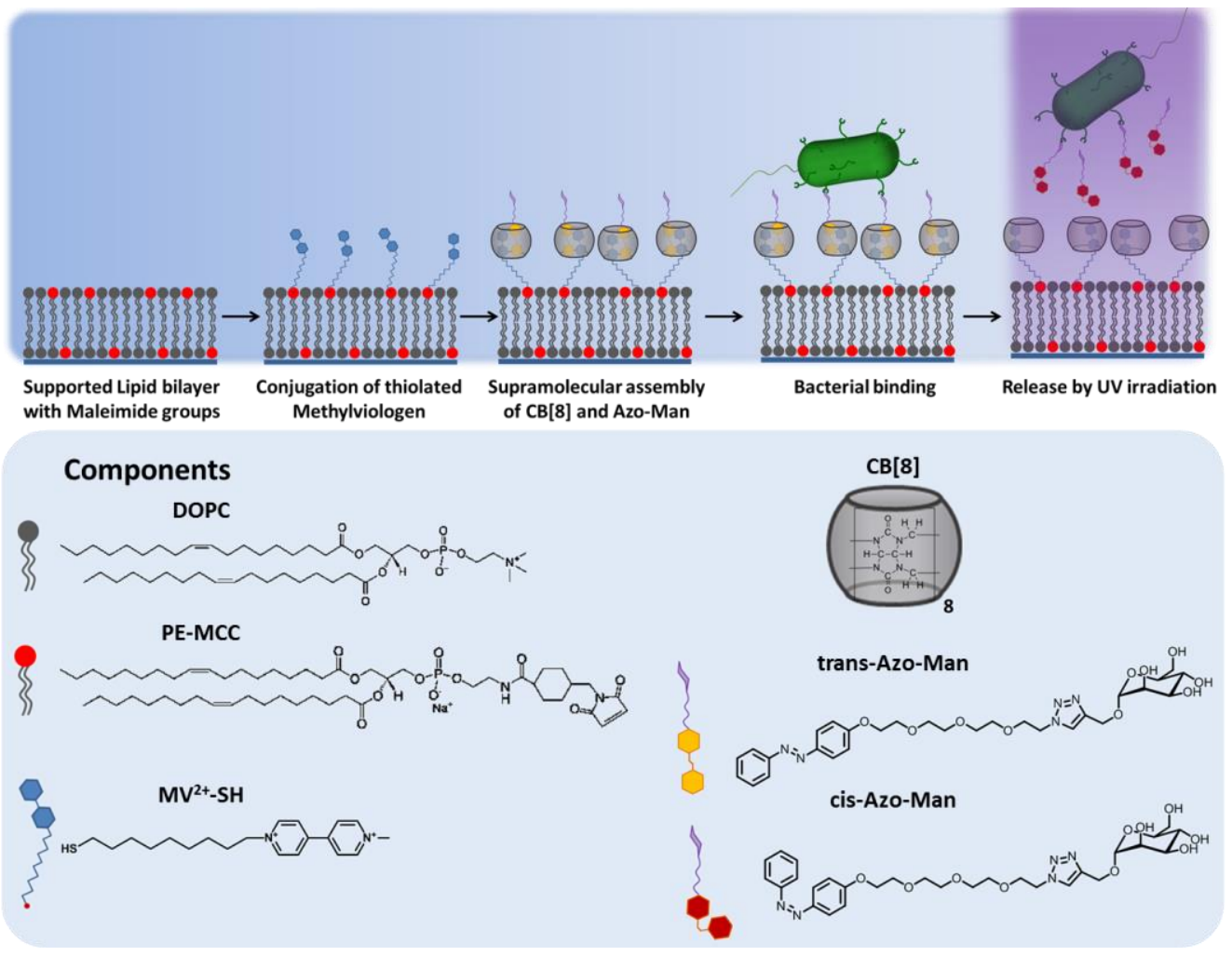

Figure 6.1. Step-wise assembly and release scheme followed by chemical structures of the individual components used. 


\subsection{Results and Discussions}

\subsubsection{Selection of a non-fouling $M V^{2+}$-functionalized layer}

In order to construct a reusable supramolecular platform for binding and release of living cells, we first needed to select a background layer with two important properties: 1) the ability to chemically incorporate methylviologen $\left(\mathrm{MV}^{2+}\right)$, the first guest molecule and 2) minimize non-specific interaction of biolomolecules and cells to the surface. To this end, we tested three potential candidates: an oligoethyleneglycol self-assembled monolayer (SAM), gel-state and liquid-state supported lipid bilayers (SLBs). We have previously reported a tetraethyleneglycol based SAM for supramolecular manipulation of mammalian cells. ${ }^{36}$ In this case, SAMs bearing maleimide groups ${ }^{37}$ were used to tether a thiolated methylviologen $\left(\mathrm{MV}^{2+}-\mathrm{SH}\right)$. The SAM was shown, to some extent, to be non-fouling and was used for mammalian cell binding and electrochemical release thereof. ${ }^{[36]}$ Alternatively, we explored the use of model membranes in the form of supported lipid bilayers (SLBs). Such SLBs, pioneered by McConnell and co-workers, consist of a two-ply sheet of lipid molecules and can mimic natural cell membranes in terms of the lateral mobility of the lipids and associated ligands. ${ }^{38,39} \mathrm{~A}$ relevant feature of SLBs is their inherent non-fouling nature. In the case of zwitterionic phosphocholinebased SLBs, negligible adsorption of various other naturally occurring proteins was observed. ${ }^{40}$ This attribute of SLBs strongly influences cell adhesion kinetics and is able to prevent cells from adhering to its surface. ${ }^{21}$ The chemical make-up of lipids e.g. the saturation of acyl chains can have a profound effect on lipid lateral mobility and phase behavior of SLBs as a function of temperature. For example, the liquid-state ( $L_{\alpha}$ phase) is characterized by a disordered packing of lipids due to the presence of unsaturated acyl chains, low melting temperature and high lipid lateral mobility, $>\mu \mathrm{m}^{2} / \mathrm{s}$. On the contrary, lipids with saturated acyl chains tend to pack more tightly in the gel-state $\left(\mathrm{L}_{\beta}\right.$ phase), increasing their $T_{m}$ and resulting in a reduced lateral mobility by at least one order of magnitude. ${ }^{41}$ In addition, chemical modification of SLBs to alter its surface properties have been shown on various accounts..$^{22,42-44}$

Although SLBs have been used in a multitude of cell studies, integrating them with supramolecular host-guest chemistry remains unexplored. Apart from their non-fouling nature, SLBs have the added advantage that they mimic the natural environment of eukaryotic cell membranes. In this study, we integrated liquid- and gel-state SLBs with supramolecular chemistry. All three aforementioned methods offered the possibility to form tightly packed layers of $\mathrm{MV}^{2+}$ at well-defined surface densities. 
We first evaluated the non-fouling nature of these types of $\mathrm{MV}^{2+}$-functionalized surfaces, i.e. SAM, liquid- and gel-state SLBs, towards biomolecules such as proteins and cells. Since $\mathrm{MV}^{2+}$ carries a double positive charge, electrostatic interactions could potentially cause non-specific interactions with biomolecules despite the non-fouling nature of the background layer. $\mathrm{MV}^{2+}$-functionalized ethyleneglycol-based SAMs on gold were made as reported previously. ${ }^{36}$ We followed the formation of gel-state and liquid-state SLBs doped with PE-MCC (maleimide-containing lipid) on $\mathrm{SiO}_{2}$ surfaces using QCM-D. In both cases, we were able to observe the conjugation of $\mathrm{MV}^{2+}$-SH onto these SLBs (Figure S6.1, Section 6.7, Supporting Information). In all cases, a 1\% surface density of $\mathrm{MV}^{2+}$ was readily obtained. Since we wanted to develop a system for bacterial binding to glycoconjugates, we chose to test the resistance of our modified surfaces against a mannose-binding lectin, Conconavalin A (ConA).

a) Liquid-state SLB

b) Gel-state SLB

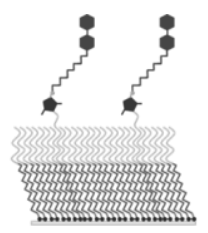

c)

MV SAM

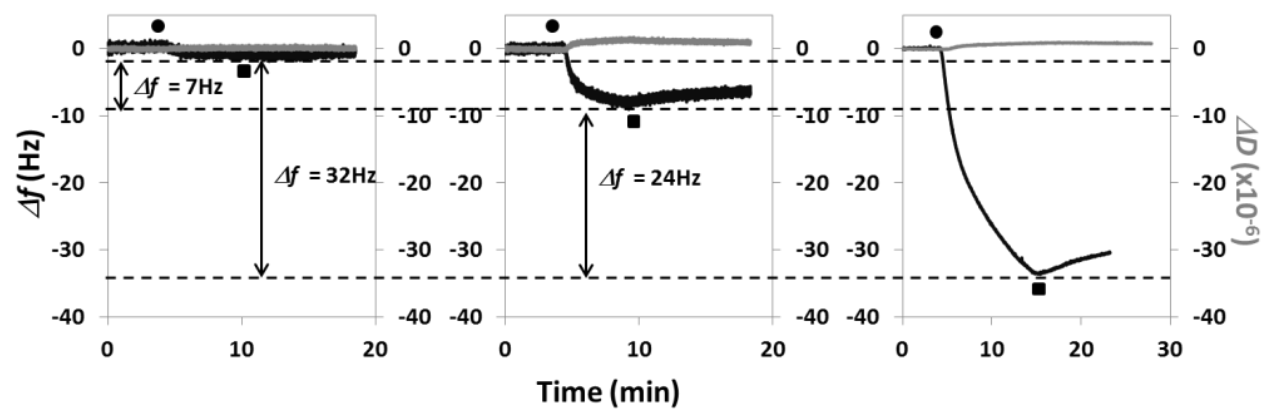

Figure 6.2. QCM-D response plots of flowing $1 \mu M \operatorname{ConA}(\bullet)$ over a) a liquid-state SLB, b) a gel-state SLB and c) an oligoethylenegylcol-based SAM, each with $1 \%$ surface density of $M^{2+}$ followed by rinsing with buffer $(\boldsymbol{\square})$. Change in frequency $(\Delta f$, black) and dissipation $(\Delta D$, grey) values correspond to the 5 th overtone.

From Figure 6.2, we clearly observed that the liquid-state SLB was the most non-fouling of the three surfaces when incubated with $1 \mu \mathrm{M}$ ConA. The non-fouling nature of SLBs behavior is often ascribed to the neutrally charged $\mathrm{PC}$ head-group over a large $\mathrm{pH}$ range, $3<\mathrm{pH}<10$. In addition, $\mathrm{PC}$ is known to attract a water layer that hampers the adsorption of proteins. In the case of liquid-state SLBs, the high lateral mobility of PC 136 
lipids further favors this protein repellent property ${ }^{40}$ It has been proposed that out-ofplane z-axis mobility of liquid-state SLB results in undulatory motions of the bilayer that further interferes in cell and protein binding. ${ }^{45}$ Similar to SAMs, gel-state SLBs and their constituents exhibit nearly no lateral mobility. Despite the absence of such out-of-plane mobility in gel-state SLBs, non-specific binding of ConA was significantly less compared to $\mathrm{MV}^{2+}$-SAMs. This difference can be ascribed to the fact that zwitterionic gel-state SLBs do however, to some extent, retain non-fouling properties. Previous QCM-D analysis of gel-state SLBs in the presence of serum showed negligible protein fouling on such surfaces. ${ }^{23}$ In addition, a cell adhesion study performed on polymerized zwitterionic SLBs consisting of Bis-SorbPC showed a high degree of cell repellence towards murine-derived macrophages despite the absence of lipid lateral mobility. ${ }^{46}$

Clearly, significant fouling was observed on gel-state SLBS and SAM PEG layers, whereas on the liquid state SLB negligible fouling was observed in the case of ConA adhesion. To further confirm that using methyl viologen functionalized gel-state SLBs and methyl viologen modified SAM PEG based layers, leads to significant non-specific adhesion of bacterial cells to these surface exceeding by far that observed on the methyl viologen functionalized liquid-state SLB, we performed bacterial adhesion tests on these three layers.

Figure 6.3 shows data of a non-specific bacterial adhesion test on a liquid-state SLB with two ratios of positively (methyl viologen-PEMCC) and negatively (TR-DHPE) charged lipid constituents. On liquid-state SLBs made of an $0.1 \%$ ratio of positively and negatively charged constituents, negligible non-specific bacterial adhesion was observed. This observation is in strong contrast with the considerable non-specific bacterial adhesion observed on liquid-state SLBs made of a lipid mixture with an excess of positively charged methyl viologen and, also, with gel-state SLBs made of an equimolar amount of methyl viologen-PEMCC and TR-DHPE. Based on these data we confirm that the balance of positive and negative charges on the layer is of utmost importance to prevent fouling of the surface by the bacteria through non-specific electrostatic interactions. We were not able to find a condition on a gel-state SLB where bacteria do not adhere. 


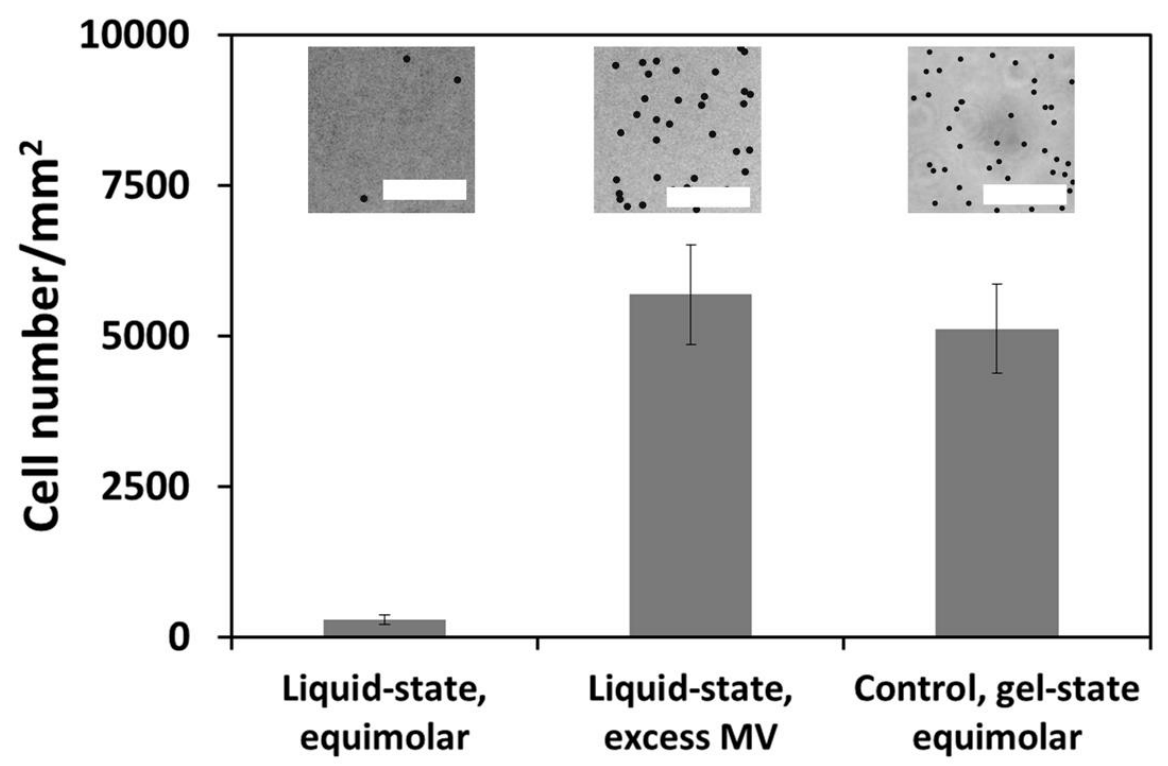

Figure 6.3. Non-specific bacterial adhesion to three lipid bilayers that were presenting methyl viologen. Liquid-state and gel-state SLBs were made of using positively $\left(\mathrm{MV}^{2+}\right.$-PEMCC) and negatively (TR-DHPE) charged lipid constituents in an equimolar amount $0.1 \%$. Liquid-state SLB using $1 \% \mathrm{MV}^{2+}$ PEMCC and 0.1\% TR-DHPE was also made and used. Bacterial cells have been falsely colored black for better visualization. Data presented as mean $\pm S T D, n=4$. Insets are sample images of the respective surfaces.

Subsequently, we attached methyl viologen to the maleimide modified SAM PEG system (Figure 6.2C). We incubated the methyl viologen with host $\mathrm{CB}[8]$, and with a mixture of $\mathrm{CB}[8]$ and second guest azobenzene modified mannose. We monitored bacterial adhesion to these four layers (Figure S6.2, Section 6.7, Supporting Information). Clearly, and as expected, the SAM with maleimide functional groups is preventing non-specific interactions with the bacteria. However, the introduction of methyl viologen on the SAM resulted, also as expected, in substantial non-specific electrostatic interactions with the bacteria, to a similar extent as observed in the case of methyl viologen functionalized gel-state SLBs in Figure 6.3. While the bacterial adhesion on the SAM including the bio-specific mannose ligands only showed a small increase in the number of adhered bacteria, performing bacterial adhesion and subsequent photo-controlled release experiments on these type of SAMs seems redundant. Then, we attempted to find a condition on a SAM PEG based surface where such non-specific interactions would be suppressed. To this end, we reacted the maleimide-functionalized SAMs with a mixture of thiol-functionalized methyl viologen 
and 3-mercapto-1-propane sulfonate (MPS), which can provide negative charges to balance the positive charges given by the incorporation of methyl viologen on the SAM. We prepared different SAMs using various ratios of these two compounds. Bacteria adhered to all of these SAMs (Figure S6.3, Section 6.7, Supporting Information). The adhered bacteria per area in the condition of methyl viologen and MPS used in a 1:3 ratio is still exceeding by large the non-specific bacterial adhesion observed on methyl viologen modified liquid state SLB made of an equimolar ratio of methyl viologen and TR-DHPE. Taken together, $\mathrm{MV}^{2+}$-functionalized liquid-state SLBs were employed to evaluate supramolecular ternary-complex formation, selective bacterial adhesion experiments and subsequent studies for photo-switchable release of adhered bacteria.

For in situ monitoring of the SLB quality during various modification steps, a fluorescent lipid-dye conjugate, Texas Red-DHPE (TR-DHPE), was included at low doping densities. A typical characterization experiment for liquid-state SLBs involves monitoring of the rate of fluorescence recovery after the SLB that has been locally bleached. Such a fluorescence recovery after photobleaching (FRAP) experiment can provide insights into lipid lateral mobility. Successful formation of a defect free $\mathrm{MV}^{2+}$ modified (liquid-state) SLB was confirmed by observing uniform fluorescence in agreement with the observations made using QCM-D. This was further complemented by the deduced diffusion coefficients, which are well within the expected range for liquid-state SLBs, i.e. $0.95 \mu \mathrm{m} / \mathrm{s}$. Texas Red-DHPE was used to monitor in situ further SLB modifications, vide infra § 6.2.2 (Fluorescence based analysis).

\subsubsection{Incorporating supramolecular components}

QCM-D based analysis

After having established a non-fouling layer grafted with $\mathrm{MV}^{2+}$, the self-assembly of the supramolecular host-guest complex was attempted. QCM-D was used to monitor the formation of the ternary complex on the $\mathrm{MV}^{2+}$-SLB. In Figure 6.4a interaction and binding of $\mathrm{CB}[8]$ is observed, as indicated by a shift in frequency and dissipation. Subsequently, a titration of Azo-Man was performed from $20 \mu \mathrm{M}$ to $1 \mathrm{mM}$, in the presence of $100 \mu \mathrm{M} \mathrm{CB}[8]$ to ensure the equilibrium is driven towards complex formation. Concentration-dependent frequency and dissipation changes were clearly observed. Washing with only $\mathrm{CB}[8]$ after the titration caused dissociation of the AzoMan and further washing with MilliQ water caused gradual dissociation of the complex. Changes in both dissipation and frequency values normally indicate that the adsorbed entities have viscoelastic properties. From the $\Delta D$ Vs $\Delta f$ plot in Figure 6.4b, we witness 
a nearly linear relationship between the dissipation and frequency during the entire titration. This indicated that both the shifts in dissipation and frequency directly correlated to the amount of Azo-Man that was binding to the surface. When the saturation values corresponding to each Azo-Man concentration was plotted against the concentration, we obtained a similar Langmuir adsorption trend from both the frequency and dissipation values. Fitting to a Langmuir adsorption equation, yielded a dissociation constant $\left(K_{d}\right)$ of $\sim 300 \mu \mathrm{M}$. This value is within an expected range for such supramolecular interactions but is higher than that previously reported by Scherman and co-workers $(\sim 70 \mu \mathrm{M})$ for $\mathrm{CB}[8]$ binding with azobenzenes in solution. ${ }^{35}$ Most likely, the surface density of $\mathrm{MV}^{2+}$ in our system is below what is required for maximum packing of $\mathrm{CB}[8]$ on the surface. The inter-ligand spacing of $\mathrm{MV}^{2+}$ molecules was estimated to be roughly $19 \mathrm{~nm}$, calculated based on the method described by Tanaka and co-workers. ${ }^{24}$ However, the outer diameter of Cucurbit[8]uril has been reported to be only $1.75 \mathrm{~nm},{ }^{47}$ nearly a tenth of the $\mathrm{MV}^{2+}$ inter-ligand spacing. Finally, the bio-activity of the self-assembled ternary complex was confirmed through the specific binding of ConA with mannose on the second guest Azo-Man. ConA specifically adhered to a liquid-state SLB modified with supramolecularly assembled Azo-Man (Figure 6.4d) when compared to the bare $\mathrm{MV}^{2+}$-functionalized SLB (Figure 6.2a). 
a) Azo-Man titration

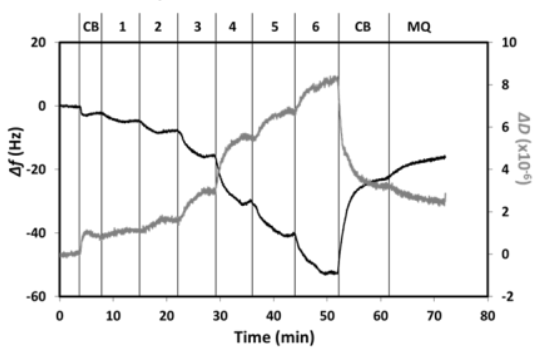

c) Azo-Man binding affinity

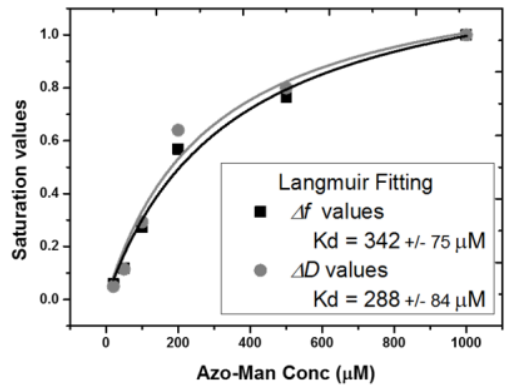

b) $\Delta D$ Vs $\Delta f$ plot

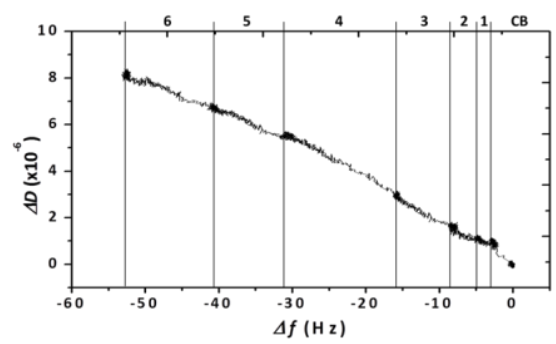

d) ConA adhesion

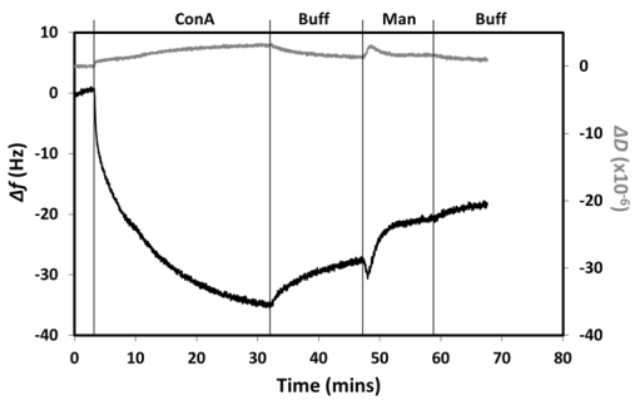

Figure 6.4. QCM-D analysis of the supramolecular assembly on liquid-state SLBs. The captions on top of the plots represent the different solutions used during the QCM-D measurement. a) Binding of $\mathrm{CB}[8]$ (CB) to an $\mathrm{MV}^{2+}$-functionalized SLB followed by titration of different concentrations of AzoMan $(1-20 \mu \mathrm{M}, 2-50 \mu \mathrm{M}, 3-100 \mu \mathrm{M}, 4-200 \mu \mathrm{M}, 5-500 \mu \mathrm{M}, 6-1000 \mu \mathrm{M})$ followed by washing with $C B[8]$ and MilliQ water (MQ). All the Azo-Man solutions also contained $100 \mu M C B[8]$. b) $\triangle D$ Vs $\Delta f$ plot for the Azo-Man titration part of Figure 6.4a. All captions above the plot are the same as in Figure 6.4a.c) Langmuir adsorption equation fitting of the saturation values from the frequency and dissipation changes seen during the Azo-Man titration. d) Adhesion of $1 \mu \mathrm{M}$ ConA onto CB[8]-AzoMan functionalized SLB followed by washing with HEPES NaCl buffer (Buff) and $10 \mathrm{mM}$ Mannose (Man). Change in frequency ( $\Delta f$, black) and dissipation ( $\Delta D$, grey) values correspond to the 5 th overtone.

\section{Fluorescence based analysis}

As mentioned previously, TR-DHPE is usually used in SLBs to evaluate the quality and fluidity of the SLB, however it can also be used to monitor modification of the SLB in situ. This lipid-dye conjugate is usually synthesized by reacting the amine of a phosphatidylethanolamine lipid with the lower aromatic ring of Texas Red sulfonyl chloride, which resides at either the ortho or para positions, ${ }^{48}$ resulting in an isomeric mixture. Interestingly, the fluorescence intensity of the ortho isomer has been shown 
to be $\mathrm{pH}$ dependent, ${ }^{49}$ with lower intensities under basic conditions. Using this phenomenon, we inspected the SLB integrity and determined the approximate lipid lateral diffusion (vide infra), which also allowed us to track the $\mathrm{MV}^{2+}$ reaction with the surface in time. The presence of the highly positively charged $\mathrm{MV}^{2+}$ attracts hydroxide ion from the water and creates a local $\mathrm{pH}$ increase. As expected, we always observed a drop in the Texas Red fluorescence intensity when $\mathrm{MV}^{2+}$-SH was conjugated to the SLB (Figure 6.5a). To ensure that this was purely a surface chemistry effect, we checked the fluorescence intensity drop after addition of an unreactive $\mathrm{MV}^{2+}$. In this case, no significant drop in the intensity was observed (Figure 6.5a). Following this, we measured the changes in fluorescence intensities as the supramolecular components were assembled. When $\mathrm{CB}[8]$ bound to the surface-immobilized $\mathrm{MV}^{2+}$, a slight rise in the fluorescence intensity was observed possibly due to the partial shielding of the charges of $\mathrm{MV}^{2+}$. When titrating with different concentrations of Azo-Man, the fluorescence intensity seems to drop at higher concentrations and seems to follow a similar trend as the binding curve in Figure 6.4c. The drop in fluorescence might be due to a change in local $\mathrm{pH}$. Also, as seen in the FRAP experiment, increased Azo-Man concentrations causes the lateral mobility to reduce, which suggests crowding of the TR-DHPE lipid resulting in self-quenching. Nonetheless, the observed trend seems to follow the binding curve presented in Figure 6.4C. Furthermore, FRAP was used to study the attachment of $\mathrm{MV}^{2+}$ to the SLB and the effect of the formation of the ternary complex on the lipid lateral mobility and diffusion coefficient (Figure 6.5c). In line with the trends observed in Figures $6.4 \mathrm{a}$ and $6.4 \mathrm{~b}$, the diffusion coefficient dropped after attachment of $\mathrm{MV}^{2+}$, increased on $\mathrm{CB}[8]$ adhesion and reduced again in the presence of increasing concentrations of Azo-Man. We tentatively interpret these observations as follows. Positively charged $\mathrm{MV}^{2+}$-lipids can possibly interact with negatively charged TRDHPE resulting in its mobility drop. Subsequently, the binding of $\mathrm{CB}$ [8], causes shielding of $\mathrm{MV}^{2+}$ allowing TR-DHPE to diffuse more freely in the bilayer. Further binding of increasing amounts of Azo-Man to the $\mathrm{MV}^{2+}-\mathrm{CB}[8]$ complex causes a drop in lateral mobility, probably since mannose, being highly hydrated, causes a crowding effect at the surface. This observed trend also seems to follow the binding curve presented in Figure 6.4C. These results highlight the use of TR-DHPE for in situ monitoring of the $\mathrm{MV}^{2+}$ reaction and assembly of the supramolecular host-guest complex. 
a) MV-SH conjugation

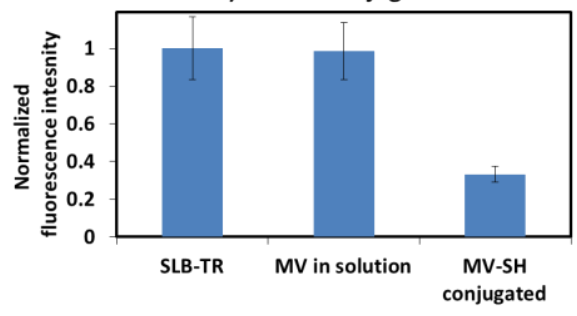

b) Supramolecular assembly

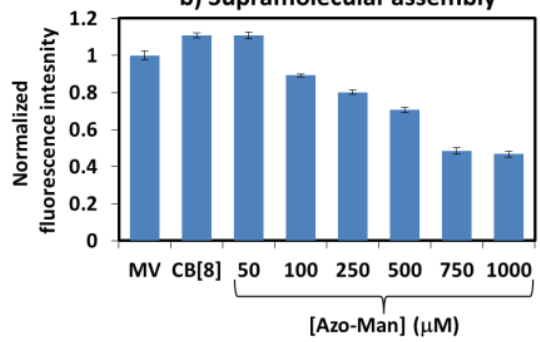

c) FRAP

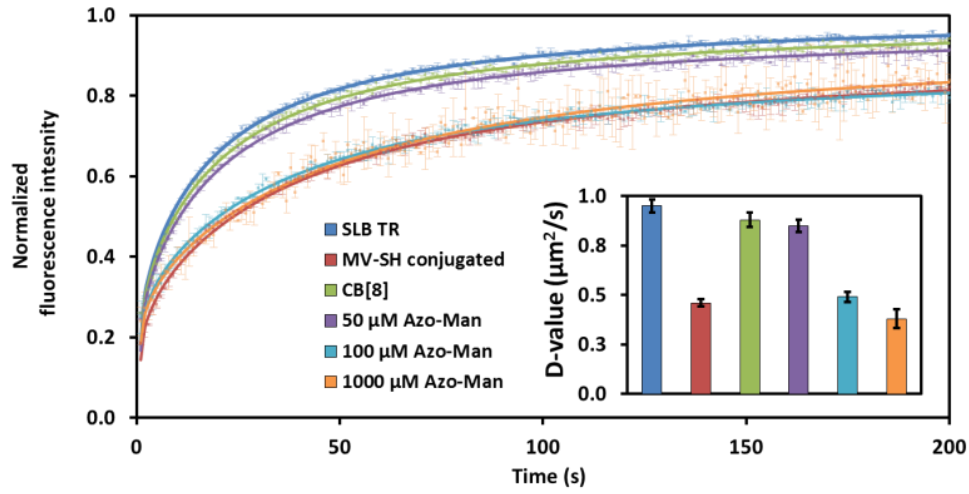

Figure 6.5. Fluorescence based analysis of the supramolecular SLBs. a) Drop in fluorescence intensity of TR-DHPE due to the conjugation of $\mathrm{MV}^{2+}$-SH with PE-MCC lipids in the SLB. All intensities have been normalized to the fluorescence due to TR in the SLB before $\mathrm{MV}^{2+}-\mathrm{SH}$ conjugation. b) Change in the fluorescence intensities seen during assembly of the $\mathrm{CB}[8]$ and Azo-Man. All Azo-Man solutions in the titration also contained $100 \mu \mathrm{MCB}[8]$. Intensity values have been normalized to the fluorescence due to TR in the SLB after $\mathrm{MV}^{2+}$-SH conjugation. c) FRAP analysis of the supramolecular assembly on the surface. Inset plot represents diffusion coefficients. The colors in the legend apply for both the fluorescence recovery curves and the diffusion coefficient plot. Data presented as mean \pm STD, $n=2$.

\subsubsection{Bacterial capture and release}

Bacterial adhesion and controls

Confident that the supramolecular assembly of $\mathrm{CB}[8]$ and Azo-Man is possible on liquidstate SLBs, we proceeded to test bacterial adhesion onto these surfaces in microfluidic channels. For this purpose, we chose to use the ORN178 and ORN208 bacterial strains. ${ }^{50}$ ORN178 is an E. coli K12 derivative expressing a native version of the FimH receptor, allowing it to specifically bind mannose. ORN208 has a genetically modified 
FimH receptor with a single point mutation and is unable to bind mannose. Apart from this, both strains are identical. When the supramolecular surface was exposed to ORN178, bacterial cells were seen adhering to the surface and did not dissociate even in the presence of strong flow $\left(300 \mu \mathrm{Lmin}^{-1}\right.$ ) (Figure 6.6). To ensure that the bacterial cells had adhered specifically to the mannose units in the host-guest system, two control experiments were performed. First, adhesion of ORN208 was tested and we observed that even at a lower flow speed $\left(15 \mu \mathrm{Lmin}^{-1}\right)$, only a negligible number of cells were visible at the surface (Figure 6.6). Similarly, when $C B[7]$ was used instead of $C B[8]$ only $\mathrm{MV}^{2+}$ was able to occupy the smaller cavity of $\mathrm{CB}[7]$ resulting in almost no ORN178 cells (less than $5 \%$ compared to the ternary complex) adhering to the surface (Figure 6.6). These experiments confirmed that bacterial adhesion was specific to the mannose presented in a supramolecular fashion using the ternary host-guest complex on SLBs. We also confirmed that $\mathrm{CB}[8]$ and Azo-Man do not affect bacterial viability by testing their growth on agar plates after being mixed together for an hour. The presence of $\mathrm{CB}[8]$ and Azo-Man did not reduce the number of bacterial colonies seen compared to the control (Figure S6.4, Section 6.7, Supporting Information). In addition, UV exposure for $5 \mathrm{~min}$ did not reduce the number of bacterial colonies compared to the control (Figure S6.5, Section 6.7, Supporting Information).

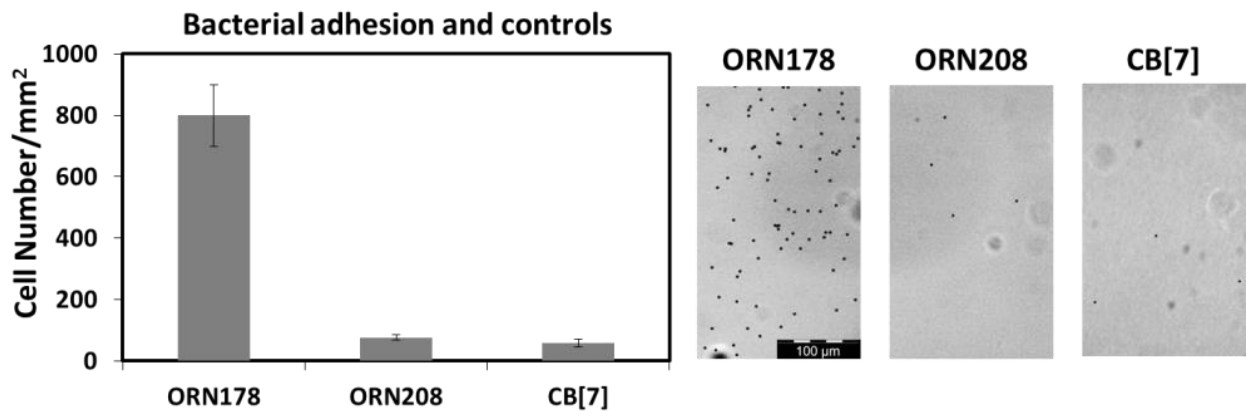

Figure 6.6. Number of bacterial cells immobilized on supramolecular SLBs. For ORN178 and ORN208 adhesion experiments, 0.1 O.D. ${ }_{600 \mathrm{~nm}}$ of the bacteria along with $10 \mu \mathrm{M} \mathrm{CB}[8]$ and $100 \mu \mathrm{M}$ Azo-Man in buffer was used. For the adhesion test of ORN178 to supramolecular SLBs containing CB[7], 0.1 O.D.60onm of the bacterial solution with $10 \mu \mathrm{MCB}$ [7] and $100 \mu \mathrm{M}$ Azo-Man was used. Data presented as mean $\pm S T D, n=6$. Bacterial cells in the images have been falsely colored black to improve visualization. 
Bacterial release due to Azo-Man photo-isomerization

Next, we tested whether the photo-isomerization of Azo-Man under UV irradiation could cause the dissociation of the bacterial cells from the surface. For this purpose, we first allowed ORN178 cells to adhere to the supramolecular SLB and then irradiated the surface with $360 \mathrm{~nm}$ UV light. At $300 \mu \mathrm{Lmin}^{-1}$ in the presence of UV irradiation, we observed rapid detachment of bacterial cells from the surface (Figure 6.7a). Interestingly, at flow speeds of $100 \mu \mathrm{Lmin}^{-1}$ and $200 \mu \mathrm{Lmin}^{-1}$, rapid dissociation was not observed. This could possibly be explained by the fact that the photo-isomerization of azo-benzenes has only around $75-80 \%$ efficiency. ${ }^{19}$ Since E. coli typically produces a few hundred FimH receptors, stronger multivalent binding to several Azo-man units is expected. Therefore higher flow speeds are needed to overcome the overall interaction strength of the remaining bound FimH and Azo-Man bonds.

Furthermore, we tested whether it would be possible to selectively release bacterial cells from a well-defined region by localized UV irradiation. Local UV irradiation was achieved by closing the field diaphragm and illuminating with $360 \mathrm{~nm}$ UV light. At a flow of $300 \mu \mathrm{L} \mathrm{min}$, , bacterial cells were seen detaching from the irradiated spot, while the vast majority of cells outside this spot remained bound (Figure 6.7b). This even occurred with simultaneous illumination with white light. This enabled real-time imaging of the photo-release as shown by the plot in the inset of Figure 6.7b (Video S6.1, Section 6.7, Supporting information). The photo-release seemed to occur rapidly within the irradiated spot while bacterial cells outside the spot seemed to stay immobilized during this period. This confirmed that localized release of immobilized bacterial cells can be easily achieved with this photo-responsive supramolecular system. 

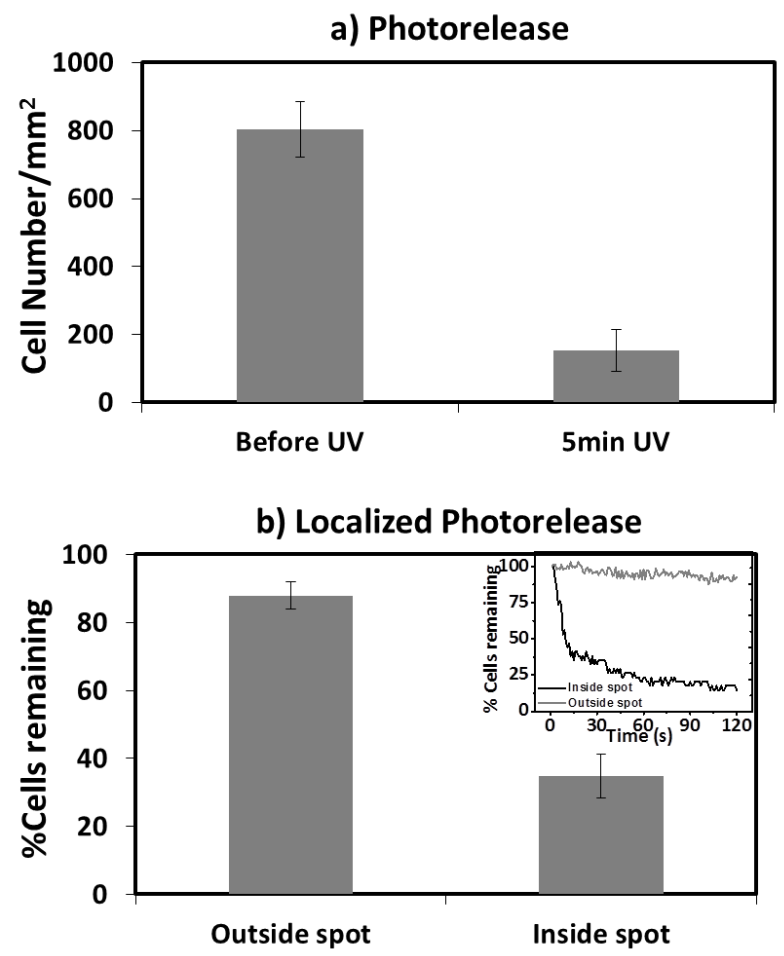

Figure 6.7. Plots representing results from bacterial release experiments by irradiation of $360 \mathrm{~nm}$ UV light in the presence of $300 \mu \mathrm{Lmin}-1$ flow. a) Before UV irradiation, cell detachment was not observed even in the presence of flow whereas 5 min UV irradiation caused detachment of nearly $80 \%$ of the cells. Error bars obtained from 3 individual release experiments. b) Localized photorelease was performed by irradiating the surface with UV light from the microscope lens using a field diaphragm. The columns represent percentage of cells remaining inside or outside the irradiated spot after 4 min irradiation. Error bars were obtained from 2 experiments with localized release done on 3 individual spots each. Inset represents the detachment of bacterial cells inside (black line) and outside (grey line) the irradiated spot as a function of time.

\subsection{Conclusions}

We have successfully demonstrated for the first time the combination of supramolecular host-guest chemistry and SLBs to develop photo-responsive supramolecular SLBs. Comparisons with other similarly possible supramolecular surfaces on SAMs and gel-state lipid bilayers indicated that liquid-state lipid bilayers were the most suitable for specific bacterial adhesion. Each step in the molecular construction of the system, including SLB formation, conjugation of the first guest, 146 
supramolecular assembly of host-guest components and photo-release have been carefully characterized using QCM-D measurements, fluorescence and bright field microscopy. Using the photo-isomerizable glycoconjugate, Azo-Man, the system has been validated for bacterial binding and release. Interesting aspects of the system including the ability to quickly monitor supramolecular assembly through fluorescence intensity changes, requirement of a certain shear stress provided by flow for bacterial photo-release and localized photo-release have been investigated. The system can potentially be used to develop reusable biosensor chips where the detection of specific pathogenic strains in a sample can be performed by capture to the surface followed by photo-release, allowing for detection in a fresh sample to be performed. Further, localized release would enable photo patterning or photo guiding of cells on the surface enabling the study of growth, migration and inter-cellular interactions.

\subsection{Acknowledgements}

The facilities of the BioNanoLab of the MESA ${ }^{+}$Institute for Nanotechnology are highly appreciated.

\subsection{Experimental Section}

Materials. All starting materials and chemicals were purchased from Sigma-Aldrich, Fluka, Serva, Becton Dickinson, Avanti Polar Lipids, Microchem, Thermo Fisher, Invitrogen and Acros organics, and they were used as received, unless otherwise stated. Thiolated Methylviologen $\left(\mathrm{MV}^{2+}-\mathrm{SH}\right)$ was synthesized as previously reported in literature. ${ }^{51}$ The Azo-Man glycoconjugate was also synthesized as previously reported in literature. ${ }^{19}$ Both ORN178 and ORN208 bacterial strains were kindly provided by Prof. Luc Brunsveld, TU/e. MilliQ water with a resistivity higher than $18 \mathrm{M} \Omega \mathrm{cm}^{-1}$ was used in all experiments.

QCM-D. Thiolated Ethyleneglycol based self-assembled monolayers bearing maleimide groups were formed on gold QCM resonators (Q-Sense) as described elsewhere. ${ }^{36}$ SLBs were formed on $\mathrm{SiO}_{2} \mathrm{QCM}$ resonators (Q-Sense). Prior to use, the resonators were cleaned with ethanol, water and with 30 mins UV-ozone treatment (UV/Ozone Procleaner plus, Bioforce Nanosciences). After mounting the cleaned resonators in the QCM-D holder, degassed $5 \mathrm{mM}$ PBS (Sigma-Aldrich, $\mathrm{pH}$ 7.4) was initially flowed. After obtaining a stable baseline (less than $\Delta 0.5 \mathrm{~Hz}$ during 10 minutes), $0.5 \mathrm{mg} / \mathrm{mL}$ large unilamellar vesicles (LUVs) were flown through the device. The $1 \mathrm{mg} / \mathrm{mL}$ stock LUV solution in milliQ was diluted 1:1 with 10 mM PBS (Sigma-Aldrich, pH 7.4) just before 
vesicle addition. For liquid-state SLBs, these steps were performed at $22^{\circ} \mathrm{C}$ and for gelstate SLBs, the temperature was raised to $45^{\circ} \mathrm{C}$. All subsequent measurements were performed at $22^{\circ} \mathrm{C}$. Subsequently, $50 \mathrm{mM}$ sodium phosphate buffer $(\mathrm{pH} 6.8)$ was passed through until a stable baseline was reached. A freshly prepared $1 \mathrm{mM} \mathrm{MV}{ }^{2+}-\mathrm{SH}$ solution in $50 \mathrm{mM}$ sodium phosphate buffer $(\mathrm{pH} \mathrm{6.8)}$ was flowed in the chamber and allowed to react for 1 hour. Assembly of the ternary complex of $C B[8]$ and Azo-Man were performed in MilliQ water using concentrations mentioned in the main text. $A 1$ $\mu \mathrm{M}$ Concanavalin A (ConA) solution in buffer (10 mM HEPES, $137 \mathrm{mM} \mathrm{NaCl}, 1 \mathrm{mM} \mathrm{MnCl}$, $1 \mathrm{mM} \mathrm{CaCl}, \mathrm{pH}$ 7.0) was flown through the device to monitor non-specific and specific binding. All fluids were exchanged continuously at $100 \mu \mathrm{L} \mathrm{min}^{-1}$ using a peristaltic pump.

PDMS flow channel. A silicon flow channel master was produced by standard photolithography steps and deep reactive ion etching. The polydimethylsiloxane (PDMS) flow channels were prepared from a degassed mixture of 10:1 Sylgard 184 elastomer and curing agent (Dow Corning Corp), which was casted onto the silicon master and cured at $80^{\circ} \mathrm{C}$ for 1 hour. The flow channels were cut to size and inlets and outlets were punched using a $1 \mathrm{~mm} \varnothing$ punch (Harris Uni-core, Sigma-Aldrich). The single straight channel had a width of $1.5 \mathrm{~mm}$ and a height of $50 \mu \mathrm{m}$.

PDMS bonding. Standard glass microscope slides (Menzel-Gläser) were rinsed and sonicated extensively with acetone, ethanol and MilliQ water, and dried prior to UVozone exposure (UV/Ozone Procleaner plus, Bioforce Nanosciences) for at least 20 mins. After UV exposure, the microscope slides were rinsed with ethanol, water and dried under a stream of nitrogen. Both cut-out PDMS flow channels and cleaned microscope slides were treated with oxygen plasma for $30 \mathrm{~s}$ at $40 \mathrm{~W}$ (Plasma prep II, SPI supplies) after which they were bonded immediately. The chips were placed on a hot plate for 10 mins at $70^{\circ} \mathrm{C}$ to increase the binding strength. Tygon tubing (VWR, 0.25 $\mathrm{mm}$ inner $\varnothing$ and $0.76 \mathrm{~mm}$ outer $\varnothing$ ) was inserted into the PDMS. The assembled $\mu \mathrm{SLB}$ flow channels were placed in an oven at $60^{\circ} \mathrm{C}$ for 1 hour. Leak-free operation was seen for flow rates up to $2 \mathrm{~mL} / \mathrm{min}$.

Vesicle preparation. 1,2-Dioleoyl-sn-glycero-3-phosphocholine (DOPC, Avanti Polar Lipids) was stored as a $10 \mathrm{mg} / \mathrm{mL}$ stock solution in chloroform at $-20^{\circ} \mathrm{C}$ and used as the main constituent in liquid-state SLBs. 1-myristoyl-2-palmitoyl-sn-glycero-3phosphocholine (MPPC, Avanti Polar Lipids) was stored as a $10 \mathrm{mg} / \mathrm{mL}$ stock in chloroform at $-20^{\circ} \mathrm{C}$ and used as the major constituent for gel-state SLBs. 1,2dipalmitoyl-sn-glycero-3-phosphoethanolamine- $\mathrm{N}$-[4-(p-maleimidomethyl)cyclohexane- 
carboxamide] (PE-MCC, Avanti Polar Lipids) was stored as a $2 \mathrm{mg} / \mathrm{mL}$ stock in chloroform at $-20^{\circ} \mathrm{C}$. The charged lipid-dye conjugate, Texas Red-1,2-dihexadecanoyl-snglycero-3-phosphoethanolamine (TR-DHPE, Invitrogen) was stored as a $1 \mathrm{mg} / \mathrm{mL}$ stock solution in methanol at $-20^{\circ} \mathrm{C}$. Desired molar ratios of the DOPC/MPPC, PE-MCC and TRDHPE were mixed and dried under a flow of nitrogen in a glass vial, and subsequently placed under vacuum overnight. The resulting lipid film was re-suspended by vortexing in MilliQ water to form multilamellar vesicles (MLVs) at $1 \mathrm{mg} / \mathrm{mL}$. The MLV solution was extruded 11 times through a $100 \mathrm{~nm}$ polycarbonate membrane (Avanti Polar Lipids). The resulting large unilamellar vesicles (LUVs) were kept at room temperature and used within two weeks. For liquid-state SLBs all these steps were performed at room temperature and for the gel-state SLBs, they were performed at $60^{\circ} \mathrm{C}$.

Supramolecular SLB formation. Supported lipid bilayer (SLB) formation was achieved by dilution of the LUV solution to $0.5 \mathrm{mg} / \mathrm{mL}$ in $10 \mathrm{~m} \mathrm{M} \mathrm{PBS} \mathrm{pH} 7.4$ (Gibco, lacking $\mathrm{MgCl}_{2}$ and $\mathrm{CaCl}_{2}$ ). Prior to LUV incubation, the channels were flushed briefly with $10 \mathrm{mM}$ PBS. The channels were then incubated with the vesicle suspension for at least 30 mins to allow for vesicle adsorption and rupture to occur. For gel-state SLBs, this step was performed at $60^{\circ} \mathrm{C}$. Subsequently, the chips were washed with an excess of MilliQ water $(300 \mu \mathrm{L} / \mathrm{min})$. From this point forth, care was taken to ensure no air bubbles entered the device. Coupling of $\mathrm{MV}^{2+}-\mathrm{SH}$ to the PE-MCC units in the SLB was done by passing $300 \mathrm{uL}$ of $1 \mathrm{mM} \mathrm{MV}^{2+}$-SH in $50 \mathrm{mM}$ Sodium phosphate buffer $(\mathrm{pH} \mathrm{6.8)}$ at 300 $\mu \mathrm{L} / \mathrm{min}$ through the channel and allowing it to incubate for $1 \mathrm{hr}$. Further assembly of $\mathrm{CB}[8]$ and Azo-Man were performed by passing 200-300 $\mu \mathrm{L}$ of each solution, as indicated in the main text, at $300 \mu \mathrm{L} / \mathrm{min}$ and allowing it to incubate in the channel for 10 mins unless stated otherwise. Experiments were done by first assembling all components and monitoring the adhesion and photo-release of bacteria and subsequently the complete supramolecular SLB was re-installed for a new experiment. We have not quantified how often this process can be done.

Fluorescence microscopy. An Olympus inverted IX71 epi-fluorescence research microscope with a Xenon X-cite $120 \mathrm{PC}$ as light source and a digital Olympus DR70 camera for image acquisition was used to acquire fluorescence micrographs of the TRDHPE in the SLBs. To this end, green excitation $\left(510 \leq \lambda_{\mathrm{ex}} \leq 550 \mathrm{~nm}\right)$ and red emission $\left(\lambda_{\text {em }}>590 \mathrm{~nm}\right)$ was filtered using the U-MWG2 Olympus filter cube. To image the FRET gradient, UV excitation $\left(325 \leq \lambda_{\mathrm{ex}} \leq 375 \mathrm{~nm}\right)$ and broad emission $\left(\lambda_{\mathrm{em}}>420 \mathrm{~nm}\right)$ was filtered. Bacterial adhesion was imaged using top bright-field illumination. In all instances ISO200 camera settings were used to record high quality, low noise images. In case of fluorescence micrographs, care was taken to ensure image acquisition was 
performed in the linear response regime. For photo-isomerization experiments, a filter cube with excitation of 350-360 nm was used with maximum illumination intensity. Localized release experiments were performed by closing the field diaphragm in the UV excitation path (epi). Brigthfield illumination enabled real-time image acquisition.

Fluorescence Recovery After Photobleaching (FRAP). FRAP measurements were conducted using a Nikon A1 CSLM with a 20x objective. To derive the diffusion coefficient, modified Bessel functions as described by Soumpasis et al. 1983, were used. Data was corrected for acquisition bleaching and normalized. FRAP data was analyzed with FRAPAnalyser (University of Luxembourg).

Bacterial cell culture. The bacterial strains ORN178 and ORN208 were grown overnight in LB media using tetracycline as the selective antibiotic. These were then spun down at $5,000 \mathrm{~g}$ for 10 mins and the supernatant was discarded. The bacteria were washed twice with $10 \mathrm{mM}$ HEPES, $137 \mathrm{mM} \mathrm{NaCl}$, pH 7 buffer by centrifugation and resuspension. Finally the bacteria were reconstituted in this buffer and their optical density at $600 \mathrm{~nm}$ was measured.

Bacterial adhesion. $\mathrm{OD}_{600 \mathrm{~mm}} 0.1$ solutions in buffer (10 mM HEPES, $137 \mathrm{mM} \mathrm{NaCl}, \mathrm{pH} 7.0$ ) were prepared of ORN178 and ORN208. Prior to flowing the bacteria through the device, $\mathrm{MnCl}_{2}$ and $\mathrm{CaCl}_{2}$ were added at final concentrations of $1 \mathrm{mM}$ each. $200 \mu \mathrm{L}$ of bacterial solution was flown through the chip at $300 \mu \mathrm{L} \mathrm{min}{ }^{-1}$. The flow was stopped and the cells were left to settle for 15 mins. After the set incubation period, flow was applied as indicated in the main text.

Data analysis. Image processing was performed using Image $(\mathrm{NIH})$ and the obtained data was analyzed and plotted using Origin (OriginLab) and Excel (Microsoft).

\subsection{References}

(1) Henderson, G., and Bradley, M. (2007) Functional peptide arrays for high-throughput chemical biology based applications. Protein Technol. Syst. Biol. 18, 326-330.

(2) Rillahan, C. D., and Paulson, J. C. (2011) Glycan microarrays for decoding the glycome. Annu. Rev. Biochem. 80, 797-823.

(3) Trevino, V., Falciani, F., and Barrera-Saldaña, H. A. (2007) DNA microarrays: A powerful genomic tool for biomedical and clinical research. Mol. Med. 13, 527-541.

(4) Jonkheijm, P., Weinrich, D., Schröder, H., Niemeyer, C. M., and Waldmann, H. (2008) Chemical strategies for generating protein biochips. Angew. Chem. Int. Ed. 47, 9618-9647.

(5) Prime, K. L., and Whitesides, G. M. (1993) Adsorption of proteins onto surfaces containing end-attached oligo(ethylene oxide): a model system using self-assembled monolayers. J. Am. Chem. Soc. 115, 10714-10721. 
(6) Houseman, B. T., Gawalt, E. S., and Mrksich, M. (2003) Maleimide-functionalized selfassembled monolayers for the preparation of peptide and carbohydrate biochips. Langmuir 19, 1522-1531.

(7) Krishnamoorthy, M., Hakobyan, S., Ramstedt, M., and Gautrot, J. E. (2014) Surface-initiated polymer brushes in the biomedical field: applications in membrane science, biosensing, cell culture, regenerative medicine and antibacterial coatings. Chem. Rev. 114, 10976-11026.

(8) Kenausis, G. L., Vörös, J., Elbert, D. L., Huang, N., Hofer, R., Ruiz-Taylor, L., Textor, M., Hubbell, J. A., and Spencer, N. D. (2000) Poly(l-lysine)-g-Poly(ethylene glycol) layers on metal oxide surfaces: Attachment mechanism and effects of polymer architecture on resistance to protein adsorption. J. Phys. Chem. B 104, 3298-3309.

(9) Dankers, P. Y. W., van Luyn, M. J. A., Huizinga-van der Vlag, A., van Gemert, G. M. L., Petersen, A. H., Meijer, E. W., Janssen, H. M., Bosman, A. W., and Popa, E. R. (2012) Development and invivo characterization of supramolecular hydrogels for intrarenal drug delivery. Biomaterials 33 , 5144-5155.

(10) Castellana, E. T., and Cremer, P. S. (2006) Solid supported lipid bilayers: From biophysical studies to sensor design. Surf. Sci. Rep. 61, 429-444.

(11) Hoa, X. D., Kirk, A. G., and Tabrizian, M. (2007) Towards integrated and sensitive surface plasmon resonance biosensors: A review of recent progress. Biosens. Bioelectron. 23, 151-160.

(12) Ronkainen, N. J., Halsall, H. B., and Heineman, W. R. (2010) Electrochemical biosensors. Chem. Soc. Rev. 39, 1747-1763.

(13) Campoccia, D., Montanaro, L., and Arciola, C. R. (2013) A review of the biomaterials technologies for infection-resistant surfaces. Biomaterials 34, 8533-8554.

(14) Mrksich, M. (2000) A surface chemistry approach to studying cell adhesion. Chem. Soc. Rev. 29, 267-273.

(15) Langer, R. (2000) Biomaterials in drug delivery and tissue engineering: One laboratory's experience. Acc. Chem. Res. 33, 94-101.

(16) Brinkmann, J., Cavatorta, E., Sankaran, S., Schmidt, B., van Weerd, J., and Jonkheijm, P. (2014) About supramolecular systems for dynamically probing cells. Chem. Soc. Rev. 43, 44494469.

(17) Boekhoven, J., and Stupp, S. I. (2014) 25th anniversary article: Supramolecular materials for regenerative medicine. Adv. Mater. 26, 1642-1659.

(18) Voskuhl, J., Brinkmann, J., and Jonkheijm, P. (2014) Advances in contact printing technologies of carbohydrate, peptide and protein arrays. Curr. Opin. Chem. Biol. 18, 1-7.

(19) Voskuhl, J., Sankaran, S., and Jonkheijm, P. (2014) Optical control over bioactive ligands at supramolecular surfaces. Chem. Commun. 50, 15144-15147.

(20) An, Q., Brinkmann, J., Huskens, J., Krabbenborg, S., de Boer, J., and Jonkheijm, P. (2012) A supramolecular system for the electrochemically controlled release of cells. Angew. Chem. Int. Ed. 51, 12233-12237.

(21) Andersson, A. S., Glasmastar, K., Sutherland, D., Lidberg, U., and Kasemo, B. (2003) Cell adhesion on supported lipid bilayers. J. Biomed. Mater. Res. A 64A, 622-629.

(22) Svedhem, S., Dahlborg, D., Ekeroth, J., Kelly, J., Hook, F., and Gold, J. (2003) In situ peptidemodified supported lipid bilayers for controlled cell attachment. Langmuir 19, 6730-6736.

(23) Andreasson-Ochsner, M., Romano, G., Hakanson, M., Smith, M. L., Leckband, D. E., Textor, M., and Reimhult, E. (2011) Single cell 3-D platform to study ligand mobility in cell-cell contact. Lab. Chip 11, 2876-2883. 
(24) Körner, A., Deichmann, C., Rossetti, F. F., Köhler, A., Konovalov, O. V., Wedlich, D., and Tanaka, M. (2013) Cell Differentiation of Pluripotent Tissue Sheets Immobilized on Supported Membranes Displaying Cadherin-11. PLoS ONE 8, e54749.

(25) Yan, X., Wang, F., Zheng, B., and Huang, F. (2012) Stimuli-responsive supramolecular polymeric materials. Chem. Soc. Rev. 41, 6042-6065.

(26) Yang, H., Yuan, B., Zhang, X., and Scherman, O. A. (2014) Supramolecular chemistry at interfaces: host-guest interactions for fabricating multifunctional biointerfaces. Acc. Chem. Res. 47, 2106-2115.

(27) Gong, Y.-H., Li, C., Yang, J., Wang, H.-Y., Zhuo, R.-X., and Zhang, X.-Z. (2011) Photoresponsive "smart template" via host-guest interaction for reversible cell adhesion. Macromolecules 44, 7499-7502.

(28) Neirynck, P., Brinkmann, J., An, Q., van der Schaft, D. W. J., Milroy, L.-G., Jonkheijm, P., and Brunsveld, L. (2013) Supramolecular control of cell adhesion via ferrocene-cucurbit[7]uril hostguest binding on gold surfaces. Chem. Commun. 49, 3679-3681.

(29) Sankaran, S., Kiren, M. C., and Jonkheijm, P. (2015) Incorporating bacteria as a living component in supramolecular self-assembled monolayers through dynamic nanoscale interactions. ACS Nano 9, 3579-3586.

(30) Pieters, R. J. (2009) Maximising multivalency effects in protein-carbohydrate interactions. Org. Biomol. Chem. 7, 2013-2025.

(31) Zhu, X.-Y., Holtz, B., Wang, Y., Wang, L.-X., Orndorff, P. E., and Guo, A. (2009) Quantitative glycomics from fluidic glycan microarrays. J. Am. Chem. Soc. 131, 13646-13650.

(32) Thomas, W. E., Trintchina, E., Forero, M., Vogel, V., and Sokurenko, E. V. (2002) Bacterial adhesion to target cells enhanced by shear force. Cell 109, 913-923.

(33) Nilsson, L. M., Thomas, W. E., Sokurenko, E. V., and Vogel, V. (2006) Elevated shear stress protects escherichia coli cells adhering to surfaces via catch bonds from detachment by soluble inhibitors. Appl. Environ. Microbiol. 72, 3005-3010.

(34) Lagona, J., Mukhopadhyay, P., Chakrabarti, S., and Isaacs, L. (2005) The cucurbit[n]uril family. Angew. Chem. Int. Ed. 44, 4844-4870.

(35) Tian, F., Jiao, D., Biedermann, F., and Scherman, O. A. (2012) Orthogonal switching of a single supramolecular complex. Nat Commun 3, 1207.

(36) Brinkmann, Jenny, Sankaran, Shrikrishnan, Rinnen, Stefan, Arlinghaus, F. Heinrich, De Boer, Jan, and Jonkheijm Pascal. Achieving well defined reversible dynamic cell interfaces through supramolecular host-guest chemistry. Manuscript under preparation.

(37) Houseman, B. T., Gawalt, E. S., and Mrksich, M. (2003) Maleimide-functionalized selfassembled monolayers for the preparation of peptide and carbohydrate biochips. Langmuir 19, 1522-1531.

(38) Brian, A. A., and McConnell, H. M. (1984) Allogeneic stimulation of cytotoxic T cells by supported planar membranes. Proc. Natl. Acad. Sci. U. S. A. 81, 6159-63.

(39) Tamm, L. K., and Mcconnell, H. M. (1985) Supported Phospholipid-Bilayers. Biophys. J. 47, $105-113$.

(40) Glasmastar, K., Larsson, C., Hook, F., and Kasemo, B. (2002) Protein adsorption on supported phospholipid bilayers. J. Colloid Interface Sci. 246, 40-7.

(41) van Meer, G., Voelker, D. R., and Feigenson, G. W. (2008) Membrane lipids: where they are and how they behave. Nat. Rev. Mol. Cell Biol. 9, 112-124.

(42) Huang, C. J., Cho, N. J., Hsu, C. J., Tseng, P. Y., Frank, C. W., and Chang, Y. C. (2010) Type I Collagen-Functionalized Supported Lipid Bilayer as a Cell Culture Platform. Biomacromolecules 11, 1231-1240. 
(43) van Lengerich, B., Rawle, R. J., and Boxer, S. G. (2010) Covalent attachment of lipid vesicles to a fluid-supported bilayer allows observation of DNA-mediated vesicle interactions. Langmuir $26,8666-72$.

(44) Huang, C. J., Tseng, P. Y., and Chang, Y. C. (2010) Effects of extracellular matrix protein functionalized fluid membrane on cell adhesion and matrix remodeling. Biomaterials 31, 7183-95. (45) Dixit, S. S., Szmodis, A., and Parikh, A. N. (2006) Glass bead probes of local structural and mechanical properties of fluid, supported membranes. ChemPhysChem 7, 1678-81.

(46) Page, J., Heitz, B. A., Joubert, J. R., Keogh, J. P., Sparer, T., Saavedra, S. S., and He, W. (2011) In vitro assessment of macrophage attachment and phenotype on polymerized phospholipid bilayers. J. Biomed. Mater. Res. A 97, 212-7.

(47) Lee, J. W., Samal, S., Selvapalam, N., Kim, H.-J., and Kim, K. (2003) Cucurbituril homologues and derivatives: New opportunities in supramolecular chemistry. Acc. Chem. Res. 36, 621-630.

(48) Daniel, S., Diaz, A. J., Martinez, K. M., Bench, B. J., Albertorio, F., and Cremer, P. S. (2007) Separation of membrane-bound compounds by solid-supported bilayer electrophoresis. J. Am. Chem. Soc. 129, 8072-8073.

(49) Jung, H., Robison, A. D., and Cremer, P. S. (2009) Detecting protein-ligand binding on supported bilayers by local pH modulation. J. Am. Chem. Soc. 131, 1006-1014.

(50) Harris, S. L., Spears, P. A., Havell, E. A., Hamrick, T. S., Horton, J. R., and Orndorff, P. E. (2001) Characterization of Escherichia coli type 1 pilus mutants with altered binding specificities. $J$. Bacteriol. 183, 4099-4102.

(51) Stoffelen, C., and Huskens, J. (2013) Size-tunable supramolecular nanoparticles mediated by ternary cucurbit[8]uril host-guest interactions. Chem. Commun. 49, 6740-6742. 


\subsection{Supporting information}

\subsubsection{Liquid- and Gel-state SLBs}

\section{SLB Formation}

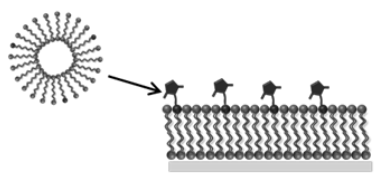

a) Liquid-state SLB

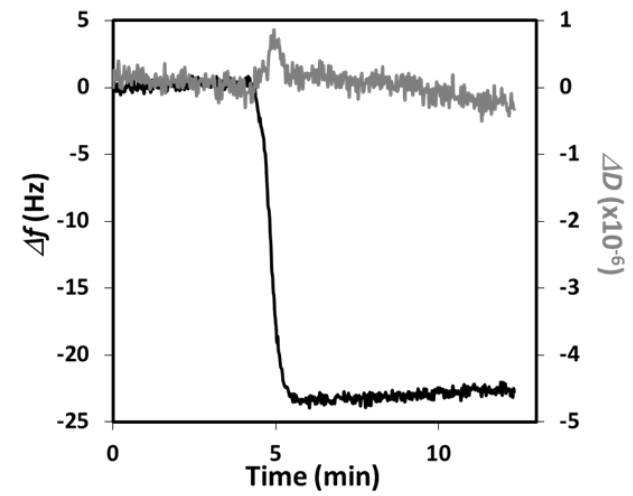

b) Gel-state SLB

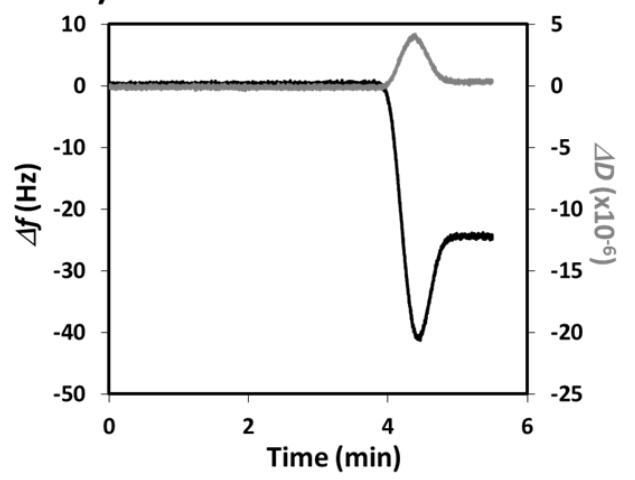

MV-SH Conjugation
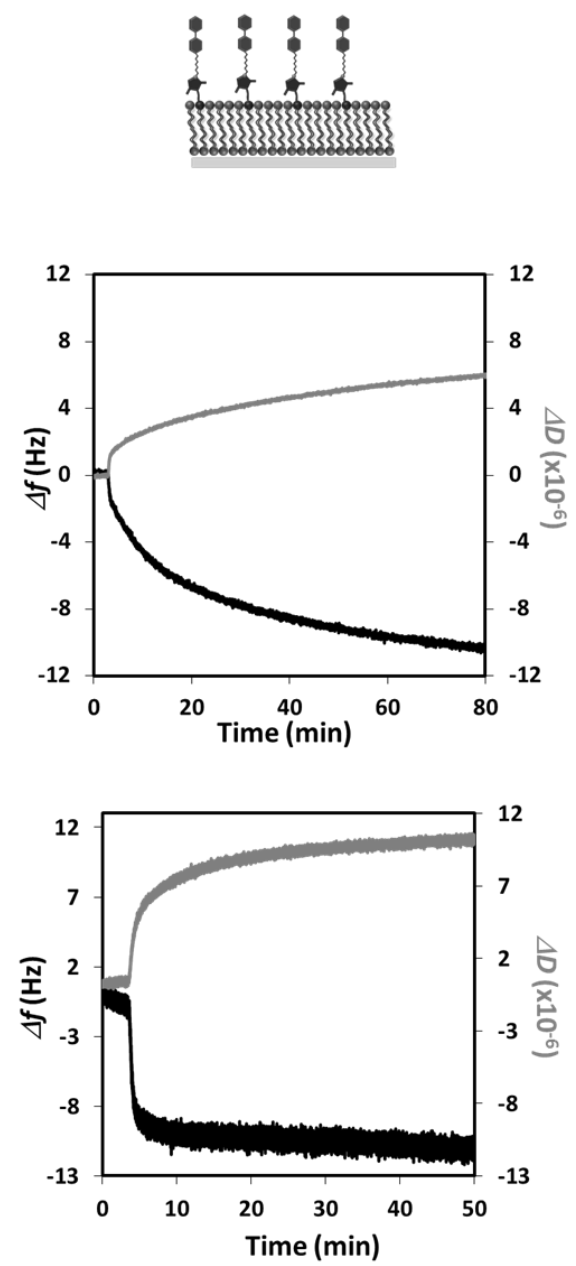

Figure S6.1. QCM-D measurements of vesicle fusion and $M V^{2+}-S H$ conjugation using a) Liquid-state SLBs and b) Gel-state SLBs. Change in frequency ( $\Delta f$, black) and dissipation ( $\Delta D$, grey) values correspond to the $5^{\text {th }}$ overtone. 


\subsubsection{Bacterial adhesion on SAMs}

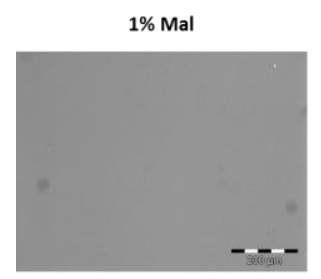

$1 \% \mathrm{MV}^{2+}+$ 100uMCB8

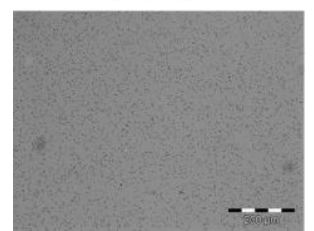

$1 \% \mathrm{MV}^{2+}$

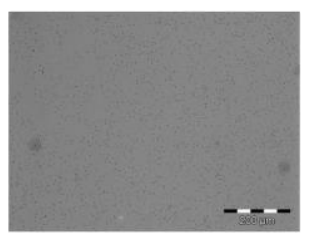

$1 \% \mathrm{MV}^{2+}+$ 100uMCB8 + 100uM Azo-Man

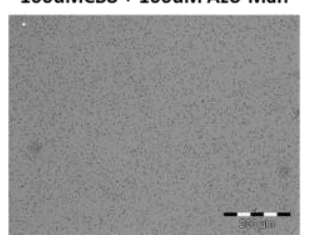

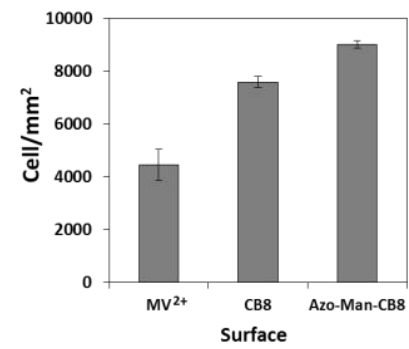

Surface

Figure S6.2. Non-specific bacterial adhesion to SAMs presenting $1 \%$ maleimide; the previous SAM reacted with thiol-functionalized methyl viologen; the previous $S A M$ incubated with $C B[8]$ or incubated with $C B[8]$ and Azo-Man. Data in graph is presented as mean $\pm S T D, n=4$.

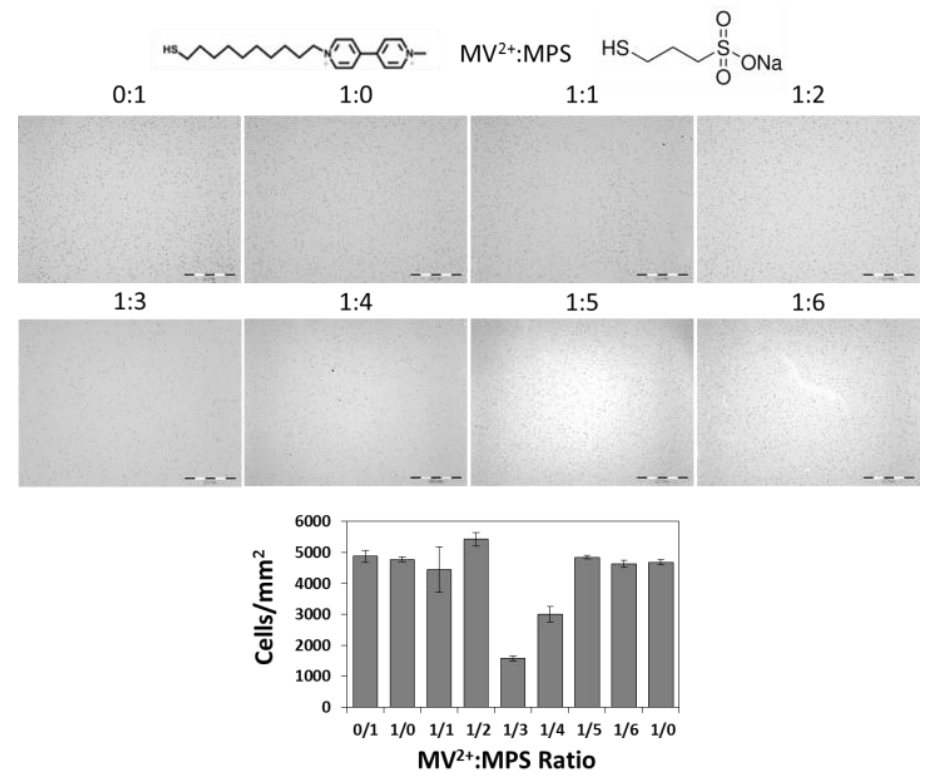

Figure S6.3. Non-specific bacterial adhesion to $1 \%$ maleimide bearing SAMs that were reacted with a mixture of thiol-functionalized methyl viologen and 3-mercapto-1-propane sulfonate (MPS). Data in graph is presented as mean $\pm S T D, n=4$. 


\subsubsection{Bacterial Vaibility}

The bacterial viability test was conducted by preparing separate solutions of bacteria having O.D.600 values of $0.1\left(\sim 10^{8}\right.$ cells $\left./ \mathrm{ml}\right)$ in buffer (10 mM HEPES, $137 \mathrm{mM} \mathrm{NaCl}, 1 \mathrm{mM}$ $\mathrm{MnCl}_{2}, 1 \mathrm{mM} \mathrm{CaCl}$ ). To one solution nothing was added and to the second $10 \mu \mathrm{M} \mathrm{CB} 8+$ $100 \mu \mathrm{M}$ Azo-Man was included. These were incubated at room temperature for $1 \mathrm{hr}$ and then $10^{5}, 10^{4}$ and $10^{3}$ cells $/ \mathrm{ml}$ dilutions were made from each of the samples. $10 \mu \mathrm{l}$ of these dilutions were placed as drops on top of LB Agar plates containing tetracycline as a selective antibiotic. The plate was then tilted to form 3 streams which quickly dried. This was then incubated overnight a $37^{\circ} \mathrm{C}$. As seen in Figure S5, both plates showed comparable number of bacterial colonies in all dilutions indicating that neither $\mathrm{CB}[8]$ nor Azo-Man had any cytotoxic effects.

a) $10 \mu \mathrm{M}$ CB8 $+100 \mu \mathrm{M}$ Azo-Man

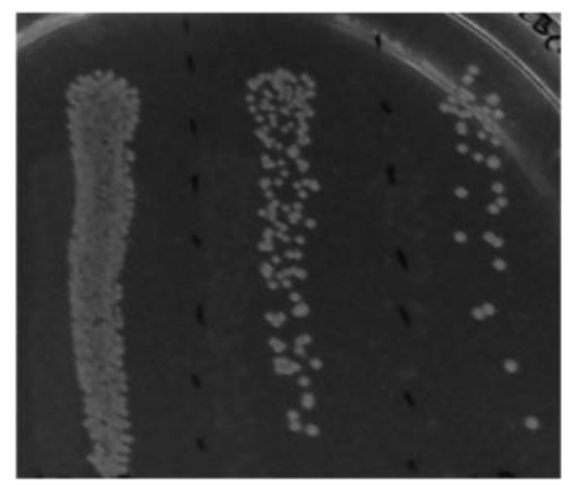

1000

100

10

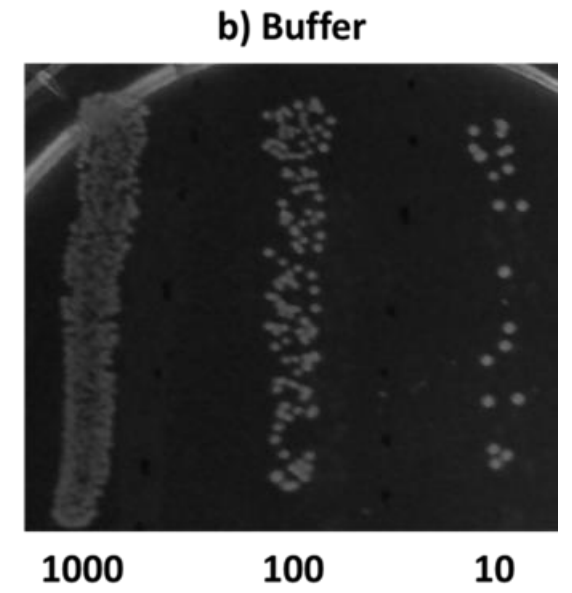

Figure S6.4. Bacterial viability test. 2 individual agar plates with colonies formed from $10 \mu$ of diluted bacterial solutions mixed with b) $10 \mu \mathrm{M} \mathrm{CB}[8]+100 \mu \mathrm{M}$ Azo-Man and b) only buffer. The values below the lanes indicate number of bacterial cells per $10 \mu \mathrm{l}$ 
a) ORN 178 minus UV $360 \mathrm{~nm}$

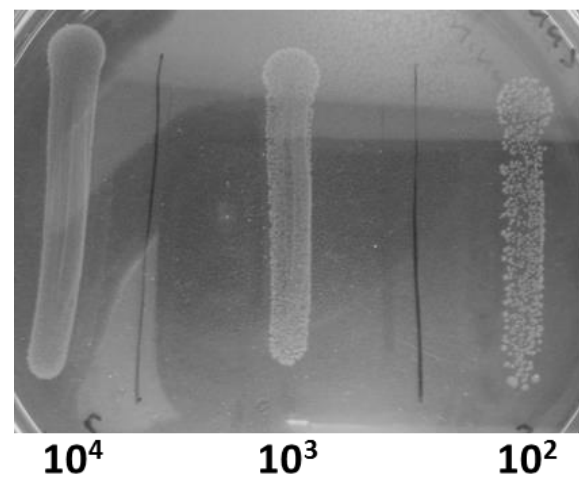

b) ORN 178 plus UV $360 \mathrm{~nm}$

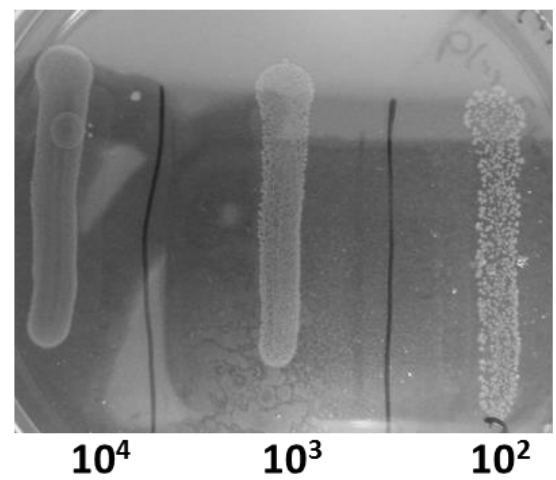

Figure S6.5. Bacterial viability test for UV exposure. OD 0.1 solutions of ORN 178 were exposed to UV $360 \mathrm{~nm}$ for 5 minutes. Agar plates with colonies formed from $10 \mu \mathrm{L}$ of diluted bacterial solutions were prepared. a) ORN 178 control plate and b) UV exposed ORN 178 cells. Dilutions indicated at the bottom. Dilutions are indicated at the bottom as cfu/lane.

\subsubsection{Video}

Video S6.1. Azo-man photo-isomerization induced release of bacterial cells from a localized spot by irradiation of 350-360 nm UV light using a field diaphragm. Irradiated spot is indicated by the dashed line and transparent violet coloring. Bacterial cells have been falsely colored blue for better visualization. Flow is from top to bottom and video spans a period of 2 minutes. The video have been provided as multimedia files and can be downloaded from the supporting information of the publication: Sankaran, S., van Weerd, J., Voskuhl, J., Karperien, M., and Jonkheijm, P. (2015) Photo-responsive cucurbit[8]uril-mediated adhesion of bacteria on supported lipid bilayers. Small doi: $10.1002 /$ smll.201502471

Link:

http://onlinelibrary.wiley.com/store/10.1002/smll.201502471/asset/supinfo/smll201502471 -sup-0002-S2.avi?v=1\&s=aboeoa69f7b1fa2f3784edcf5cfoac4941e $394 \mathrm{~d} 6$ 


\section{Chapter 7}

\section{Probing Threshold Binding of E. coli to Continuous Mannose Gradients under Flow}

In pathogenic infections, cell-cell recognition has been shown to occur through specific interactions between carbohydrates on the host cell-surface and complementary lectins on opposing microbial cells. The initiation of such recognition events is critically dependent on the carbohydrate surface density and is often resistant to shear forces, which are commonly experienced in bodily fluids. To probe these factors simultaneously, continuous gradients of mannose ligands were electrochemically generated on locked-in supported lipid bilayers (SLB) in a microchannel. Using this platform, specific E. coli ORN178 adhesion ( $K d$ of $0.9 * 10^{-21} \mathrm{M}$ ), detachment and displacement was shown to depend on the mannose-density and shear stress. The results demonstrate that our chipbased method is a powerful bio-analytical tool to provide insights into multivalent cellular binding events and to further our understanding of early stages of infection.

This work in this chapter was done in collaboration with Dr. Jasper van Weerd and is part of the manuscript: J. van Weerd," S. Sankaran," O. Roling, S. Sukas, B-J. Ravoo, S. O. Krabbenborg, J. Huskens, S. le Gac, M. Karperien and P. Jonkheijm, Probing the threshold in bacteria-mannose recognition using locked-in supported lipid bilayer gradients, Submitted to Small, 2015

\# Equal Authorship 


\subsection{Introduction}

Living cells are constantly exposed to a variety of external chemical stimuli, such as hormones, growth factors and virulence factors, which are received either in soluble form from extracellular fluids, or through direct cell-cell contacts. ${ }^{1,2}$ Cell-surface receptors can interact with molecular signals on opposing cells via specific binding to ligand molecules. In nature, biochemical mechanisms exist to translate that binding into a cellular response. ${ }^{3,4}$ In this way, intercellular interactions can be as diverse as the binding of pathogens to their target tissues, sperm-egg binding, interactions among cells in the immune system, and recognition among cells during embryonic development. $^{5-7}$ Numerous techniques have been developed to improve our understanding of the molecular basis of cell-cell recognition and have consequently made significant implications for improved intervention in many areas of biology and medicine. ${ }^{5,8,9}$ In the case of pathogenic infections, it has been shown that some microbes latch onto target cells using lectins that specifically bind different cell-surface carbohydrates such as mannose. ${ }^{10}$ These interactions have evolved to occur in a multivalent manner. Apart from being dependent on ligand density, some lectins utilize catch bonds when binding to carbohydrates, which means that the binding strength is not only determined by the ligand density but also by shear stresses that the cells experience. For example, binding of uropathogenic E. coli via their fimbrial proteins FimH with mannose that reside on the mammalian cell surfaces is strengthened with higher shear stress. As a result, the bacterium is able to mitigate the stresses from bodily fluid flow and cause infection. ${ }^{11,12}$ Glycan arrays have been extremely successful in determining the specificity and affinity of pathogenic lectins towards carbohydrates, however the effect of glycan surface density is more difficult to analyze. ${ }^{13-16}$ Recently, more intricate platforms have been proposed for the study of bacterial binding thereby exploiting the mannose-FimH interaction. Here, a supramolecular host-guest complex was assembled on adamantyl-modified graphene using mannose-cyclodextrin. The multivalent presentation of mannose resulted in strong binding of E. coli. Upon addition of a competitor, the surface-bound host-guest complex could be disassociated resulting in controlled release of the bacterium. ${ }^{17}$ Usually strategies require the preparation of several arrays or surfaces with discrete ligand densities, a cumbersome process prone to inaccuracies while the information is biased by the pre-selected ligand concentrations. ${ }^{13-15}$ Surface-based continuous gradient systems integrated with microfluidics are ideal systems to provide insights in the threshold value of the ligand surface density for pathogen recognition under physiologically relevant conditions. In the present work, supported lipid bilayers (SLB) are used to prepare stable mannose 
gradients for interaction studies with fimbrial FimH receptors found on uropathogenic E. coli strains. SLBs are advantageous for cellular studies due to their non-fouling nature and ease of chemical functionalization. ${ }^{18-23}$ Previously, we reported a method to generate stable 'locked-in' gradients using zwitterionic gel-state SLBs, that display negligible mobility at room temperature $\left(<10^{-3} \mu \mathrm{m}^{2} / \mathrm{s}\right)$, and chemically modified them. ${ }^{24}$ This chip-based system also allows us 1) to prepare continuous gradients, 2) to modify them with carbohydrate binding ligands and 3) to probe the effect of shear stress on binding of E. coli by varying the flow speed. To prepare mannose gradients (Figure 7.1a) $\mu S L B$ electrophoresis chips (Figure S7.1, Section 7.6, Supporting Information) were used that have interdigitated gold electrodes (spaced $500 \mu \mathrm{m}$ ), $\mathrm{Cr}$ corrals (spaced 100 $400 \mu \mathrm{m})$ and are equipped with a PDMS flow channel $(5,000 \times 500 \times 50 \mu \mathrm{m})$. The chips were first modified with a gel-state SLB, based on DPPC $\left(T_{m} 41^{\circ} \mathrm{C}\right)$, by means of vesicle fusion at $60^{\circ} \mathrm{C}$ (Figure 7.1a, Step 1). The high $T_{m}$ of DPPC particularly provides a convenient temperature window in which no lateral mobility was observed. Included in the vesicles were DPPE lipids, which were used for subsequent chemical modification, and fluorescent and negatively charged TR-DHPE lipids, which were used for visualization of the gradients. After cooling the chip to room temperature $\left(T<T_{m}\right)$ the amine group of DPPE-lipids in the SLB was reacted with the heterobifunctional sulfoSMCC crosslinker and subsequently with a thiol functionalized mannose (mannose-SH) to yield mannose-functionalized SLBs (Figure 7.1a, Step 2). ${ }^{25}$ Upon heating the device above the $T_{m}$ once more, $\mu S L B$ electrophoresis was performed resulting in electrophoretic migration of single negatively charged mannose-modified DPPE lipids and TR-DHPE (Figure S7.2, Section 7.6, Supporting Information). After 30 minutes of electrophoresis and continuous flowing liquid in opposed direction, the chip was rapidly cooled down to room temperature to lock the gradients in time (Figure 7.1a, Step 3). To evaluate the binding characteristics of bacteria, the mannose gradients were incubated with E. coli strain ORN178, expressing wild type FimHK514, and control strain ORN208, expressing a mutated inactive form of FimHK514 (Figure 7.1a, Step 4). The binding behavior of the ORN strains was further probed by monitoring the detachment of bound bacteria upon increasing the flow speed and thus shear stress (Figure 7.1a, Step 4). 


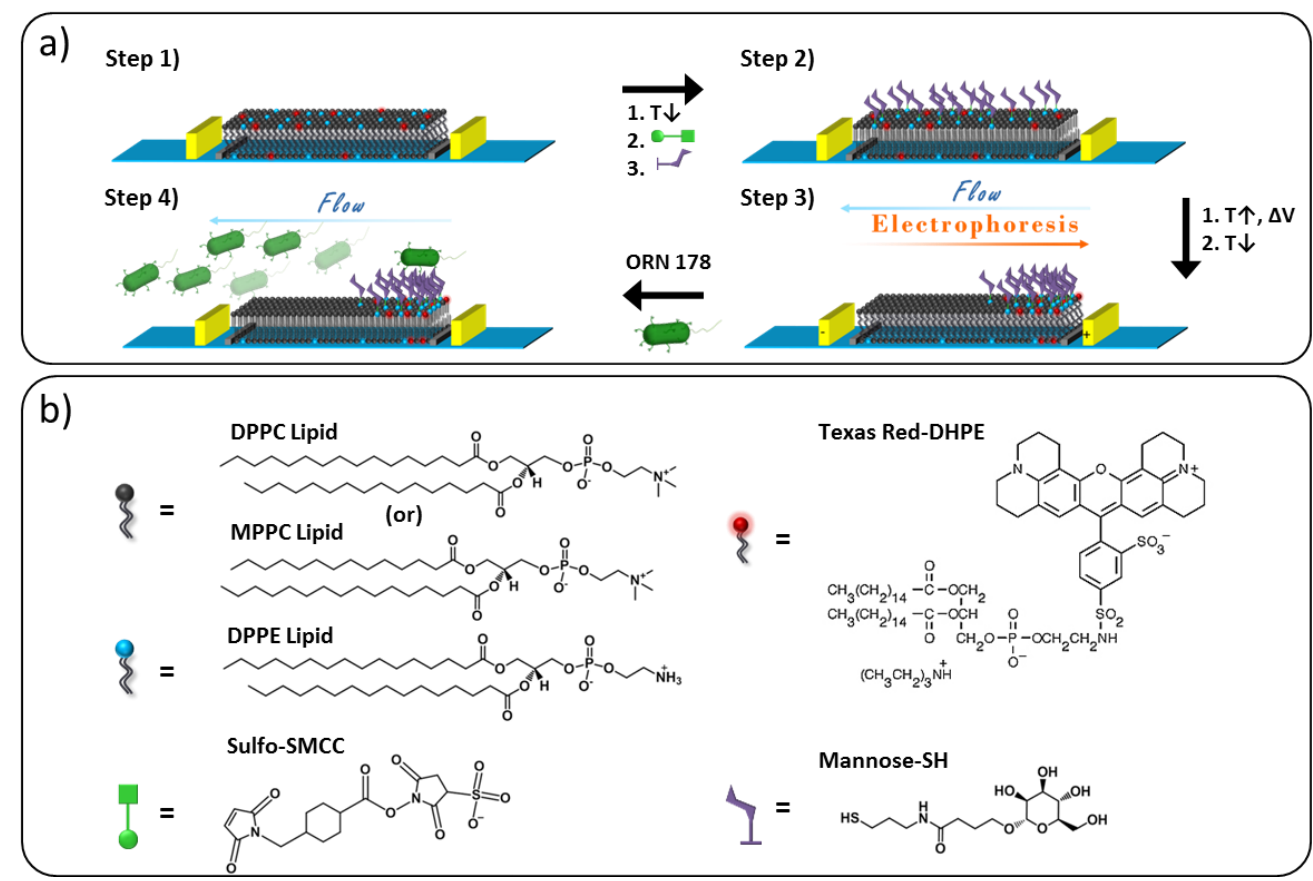

Figure 7.1. a) The steps to generate SLB-based mannose gradients for bacterial binding. (see text for details) and b) chemical structures of the corresponding molecular components.

\subsection{Results and Discussions}

\subsubsection{QCM-D characterization}

First, the modification of the SLB with mannose was validated using quartz-crystal microbalance with dissipation monitoring (QCM-D). To this end, borosilicate-coated QCM resonators were modified with either a gel-state MPPC-based SLB $\left(T_{m} 35^{\circ} \mathrm{C}\right)$ or a liquid-state DOPC-based SLB. MPPC was used instead of DPPC since it yielded a more stable QCM-D response due to the possibility of using a lower temperature for SLB formation. The liquid-state SLB system was included as a comparison since it was possible to perform all the steps at room temperature, which is ideal for the QCM measurements. The SLBs were also doped with 0.2 mol\% TR-DHPE and 1 mol\% DPPE. The characteristic response of vesicle fusion leading to the formation of the SLB was observed, yielding a $-25 \mathrm{~Hz}$ frequency change $(\Delta f$ ) (Figure $7.2 \mathrm{a}, \mathrm{d}$ ), which is in agreement with literature. ${ }^{26}$ Both the reaction of sulfo-SMCC and the subsequent conjugation of mannose-SH resulted in changes in frequency $\Delta f$ and dissipation $\Delta D$ 
(Figure $7.2 \mathrm{~b}, \mathrm{c}, \mathrm{e}, \mathrm{f}$ ). These changes stabilized after 30 and $50 \mathrm{~min}$, respectively, indicating that the reaction was complete. Larger shifts in $\Delta D$ and $\Delta f$ were observed after coupling of mannose-SH, which is presumably related to a more flexible, polar and extended structure involving additional coupled water. ${ }^{27}$ However it must be noted that unexpectedly large $\Delta f$ and $\Delta D$ changes were observed during this step in the case of gel-state SLBs. We attribute this to artifacts generated by measuring modifications on SLBs in the gel-state since liquid-state SLBs did not show this behavior (Figure 7.2f).
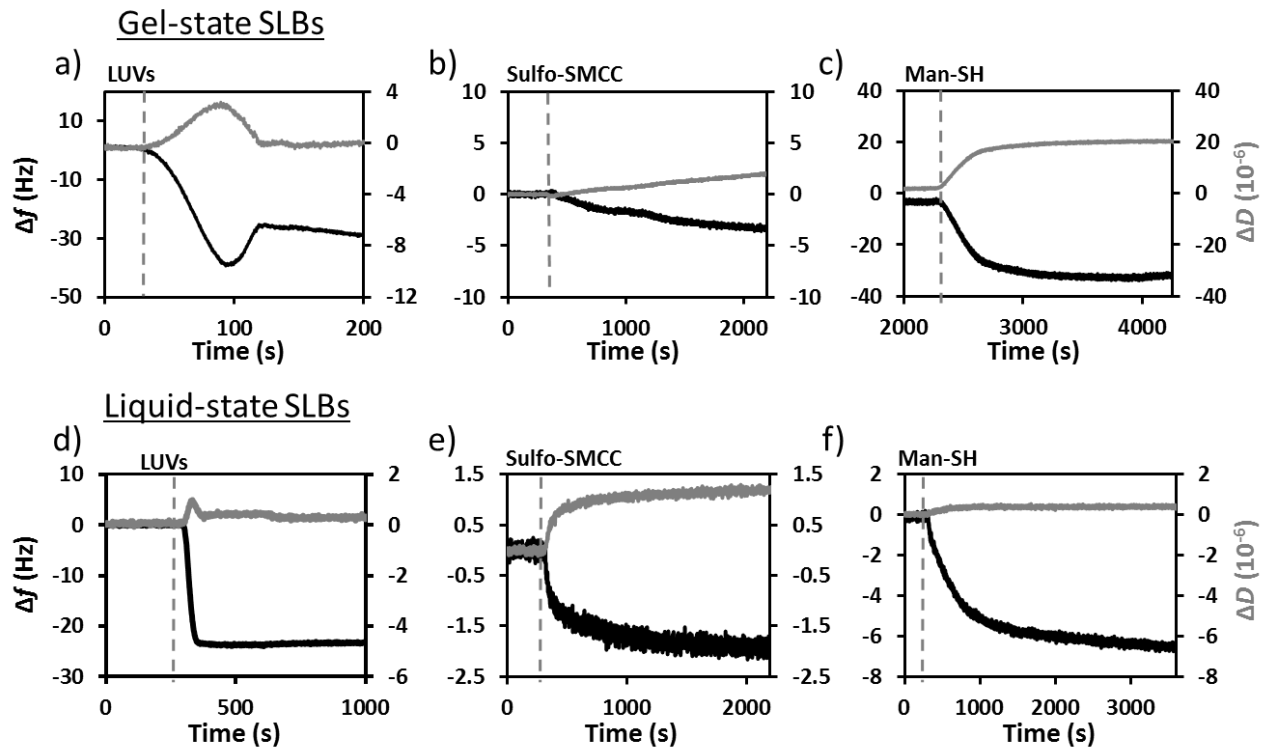

Figure 7.2. Evaluating mannose-modified SLB with QCM-D. Changes in resonant frequency $(\Delta f$, black) and dissipation ( $\triangle D$, grey) were followed during the formation of SLBs doped with $1 \mathrm{~mol} \%$ DPPE and 0.2 mol\% TR-DHPE - a) MPPC-based SLB at $45^{\circ} \mathrm{C}$ and d) DOPC-based SLB at $22^{\circ} \mathrm{C}$. b), e) Reaction of $5 \mathrm{mM}$ sulfo-SMCC and c), f) $1 \mathrm{mM}$ mannose-SH (Man-SH) at $22^{\circ} \mathrm{C}$ in all cases.

The binding properties of the mannose-modified SLBs were assessed by flowing a $1 \mu \mathrm{M}$ solution of lectin protein Conconavalin A (ConA) (Figure 7.3). ConA is homotetrameric lectin that is able to bind surface immobilized mannose. ${ }^{28}$ Binding of ConA to the mannose-modified SLB occurred within $15 \mathrm{~min}$ and only slowly dissociated during rinsing with buffer (Buff). Upon introduction of a $10 \mathrm{mM}$ mannose solution (Man), fast dissociation of ConA from the gel-state SLB was noted. Slightly larger $\Delta f$ and considerably larger $\Delta D$ shifts were observed in with the gels-state SLBs (Figure 7.3a) compared to the liquid-state SLBs (Figure 7.3b). The higher dissipation change indicates 
larger viscoelastic effects in gel-state SLBs on the assembly of additional layers of molecules. No ConA binding was observed to unmodified MPPC-SLB doped with DPPE and TR-DHPE, confirming specific interaction and the non-fouling nature of SLBS (Figure S7.3, Section 7.6, Supporting Information).
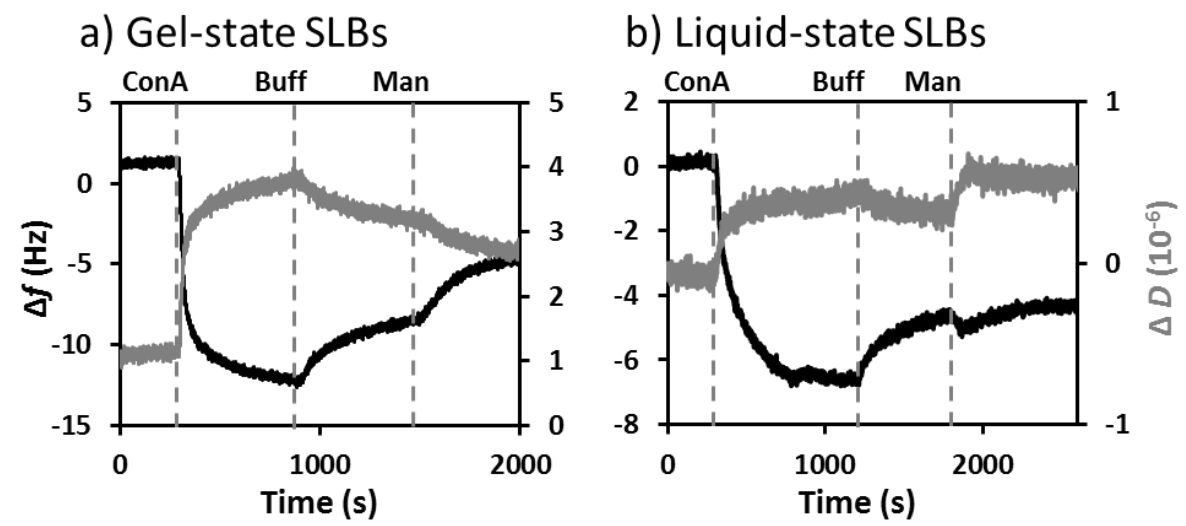

Figure 7.3. QCM-D response ( $\Delta f$ - black, $\Delta D$ - grey) of ConA adhesion and dissociation on a) gel-state SLBs and b) liquid-state SLBs.

The QCM-D experiments confirmed the in situ formation of DPPE-mannose and its specific interaction with ConA.

\subsubsection{Bacterial adhesion to mannose gradients}

To study $E$. coli binding, mannose-SLB gradients were prepared and incubated with $E$. coli strain ORN178 or control strain ORN208. Specific binding of ORN178 was observed across the device after flushing the surface to remove non-adherent cells (Figure 7.4a). The electrophoretic build-up of DPPE-mannose was deduced with TR-DHPE. ${ }^{29}$ SLBs were compartmentalized into different sized corrals and during gradient formation, the highest buildup of TR-DHPE was observed in the 210 and $430 \mu \mathrm{m}$ long corrals (Figure 7.4b). The $430 \mu \mathrm{m}$ long corrals allowed for the formation of a more gradual gradient, which enabled the analysis of more bacterial cells within individual ranges of mannose surface density. All further analysis was carried out based on bacterial behavior within this corral. 
a)

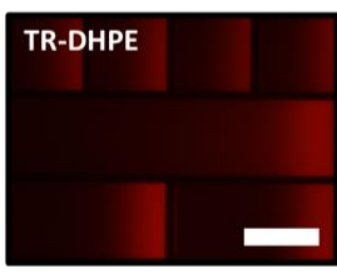

b)
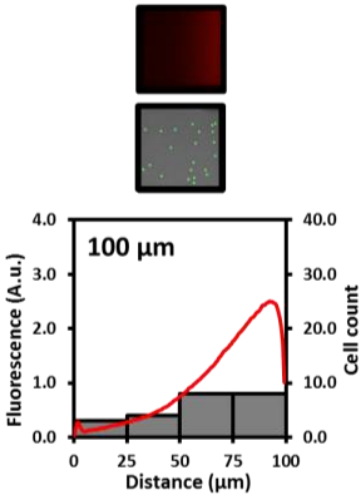
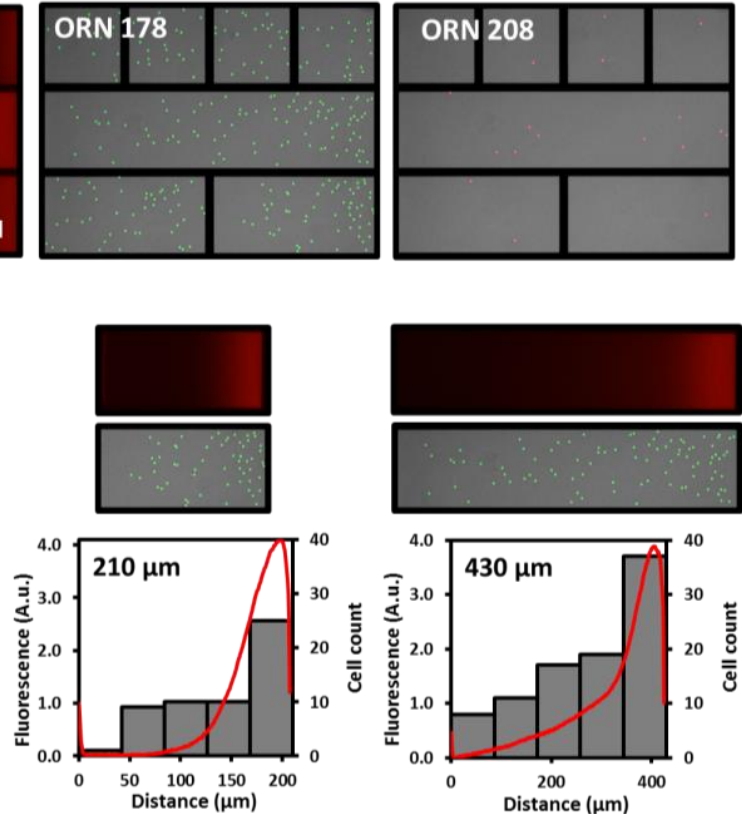

Figure 7.4. Specific binding of ORN178 to the mannose gradients on locked-in SLBs. a) Fluorescence microscopy image of the mannose gradient (TR-DHPE) and bright field microscopy images of ORN178 and ORN208 binding. The bacteria are given a false color to guide the eye i.e. ORN178 green and ORN208 red. Negligible binding of ORN208 was observed. b) Density dependent binding of ORN178 to all the corrals, i.e. 100, 210 and $430 \mu \mathrm{m}$ in length, was noted after washing away non-adherent cells. Top - the respective fluorescence and bright field images after ORN178 binding. The bacteria are quantified in bins and plotted against the fluorescence of TR-DHPE in the gradient. Scale bar 100 $\mu \mathrm{m}$.

In Figure 7.5a the bacterial binding to the mannose gradient is shown for the $430 \mu \mathrm{m}$ long corral. The adherent bacteria are given a false color and are binned. The TR-DHPE fluorescence image in Figure 7.5a exhibits a distinct exponential fluorescence build-up along the corral. Only ORN178 shows a clear density dependent binding with the gradient (Figure 7.5b) whereas only negligible binding of control ORN208 was noted i.e. ca. 5\% compared to ORN178 under flow conditions (Figure 7.5C). Control experiments performed on unmodified DPPC-based SLBs doped with DPPE and TRDHPE revealed no interaction between the cells and the zwitterionic gel-state SLBs (Figure 57.4, Section 7.6, Supporting Information), suggesting specific interaction of the FimH receptor with DPPE-mannose in the gradient. By varying the doping density of DPPE, i.e. $0.4,2$ and 4 mol\%, a quasi-binding plot for ORN178 could be deduced (Figure 
7.5d). A significant rise in the number of adhered bacteria by an order of magnitude within a very narrow window of rising mannose density is clearly seen. Beyond $1 \mathrm{~mol} \%$ mannose surface density, the number of adhered bacterial cells remains constant. Based on the TR-DHPE fluorescence and DPPE-doping density, the average inter-ligand spacing and thus mannose density was deduced. ${ }^{30}$ The binding data was fitted to the Hill equation and yields a $K_{d}$ of $0.9 \times 10^{-21} \mathrm{M}$, similar to the value reported by Guo and coworkers i.e. $1.0 \times 10^{-21} \mathrm{M} .{ }^{31}$ Interestingly, it was not possible to fit the plot with the Hill equation having an n-value of 1 (Figure S7.5, Section 7.6, Supporting Information). Allowing the fitting to be done with a variable $n$-value resulted in $n=4.2$. These characteristics, combined with the fact that a the $K_{d}$ value of a single FimH-mannose interaction is reported to be in the $\mu \mathrm{M}$ range, ${ }^{15}$ indicates that the bacteria interact with the gradient surfaces in a multivalent manner. This is expected since the bacterial surface has a few 100 type 1 fimbriae that can interact with surface immobilized mannose units. These experiments demonstrate that continuous SLB gradient chips can be easily employed for specificity and binding studies. ${ }^{3}$
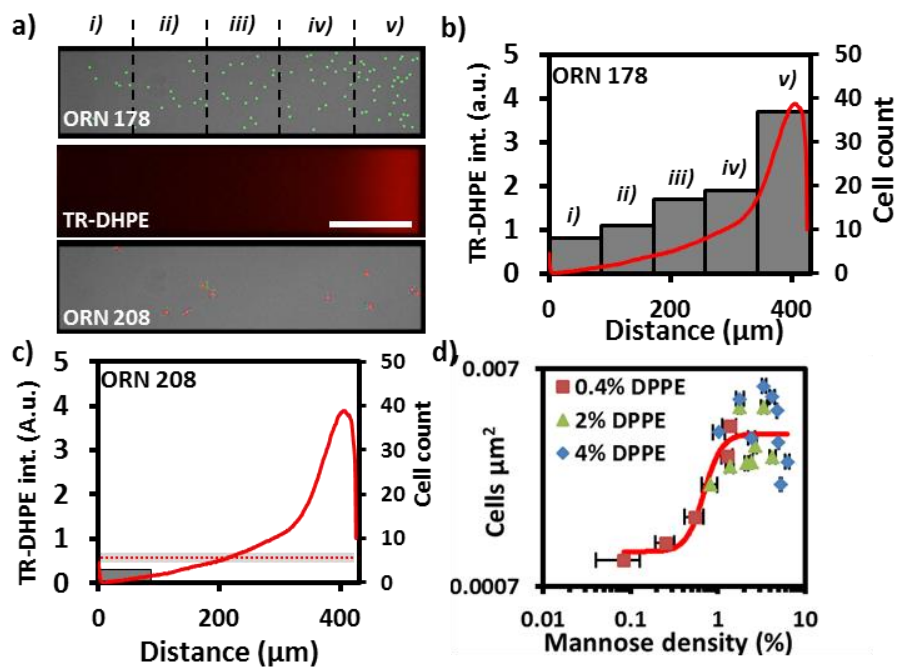

Figure 7.5. Bacterial gradient on 0.4 mol\% DPPE-mannose, $430 \mu \mathrm{m}$ long corral. a) Bright field micrographs of ORN178 (green) and ORN208 (red) binding at 100 dynes cm-2, scale bar $100 \mu \mathrm{m}$. Cell density vs. TR-DHPE fluorescence of b) ORN178 and c) ORN208. d) ORN178 binding plot fitted to Hill (solid red line).

In addition, since our chip is equipped with a flow channel, the effect of shear stress on these binding characteristics can be simultaneously analyzed. To that end, the flow 
speed was varied while monitoring detachment and displacement of the bacterial cells with microscopy (Figure 7.6). To accurately describe the shear stress near the surface, a finite element simulation was performed (Figure 57.6, Section 7.6, Supporting Information). In our study, shear stress values of up to ca. 200 dynes $\mathrm{cm}^{-2}$ were evaluated. This range of values is physiologically relevant, since in vivo, wall shear stress in human blood vessels range from a tenth, to several hundreds of dynes $\mathrm{cm}^{-2}$ for e.g. veins and stenotic vessels, respectively. ${ }^{32}$

In the region of high bacterial cell density (bin $v$ ) driven by the high surface density of mannose, the majority $(80 \pm 10 \%)$ of ORN178 cells were able to remain bound under increasing shear stress (Figure 7.6a). In the two regions with the lowest density of mannose (bins $i$ and ii) a significant reduction $(28 \pm 3 \%$ ) of bound bacterial cells was observed while bins iii and iv represented an intermediate case ( $61 \pm 3 \%)$. In the absence of flow and in the presence of cells, specific interaction of ORN178 with the mannose surface resulted in a twice higher initial cell density compared to ORN208 (Figure 7.6b). When these ORN208 cells were subjected to flow with increasing shear stress, ca. 95\% of the initially adhered bacteria were washed away (Figure 7.6c).

a)

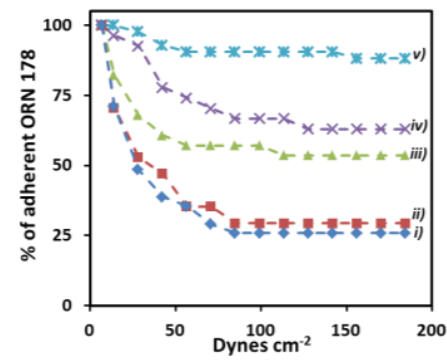

b)

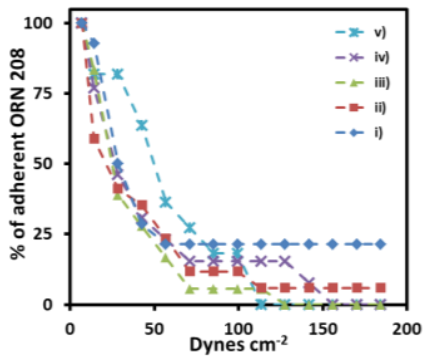

c)

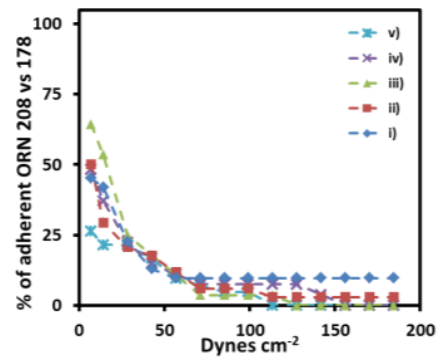

Figure 7.6. Influence of shear stress on bound ORN178 and ORN208 cells to a $0.4 \mathrm{~mol} \%$ DPPEmannose gradient. a) Percentage of adherent ORN178 vs. shear stress. b) Percentage of adhered ORN208 subjected to varying shear stresses. c) Percentage of adhered ORN208, normalized against ORN178 data to highlight the difference in surface density, subjected to varying shear stresses. Cells were counted in 5 bins with increasing mannose surface density, i to $v$ respectively.

In all cases, bound bacterial cells moved across the surface in the flow direction each time promptly after the flow speed was increased. In the case flow speeds were kept constant, no movement was observed. This result is in agreement with a previous report in literature where $E$. coli movement in time was exclusively observed at very 
low shear stress, i.e. $<10$ dynes $\mathrm{cm}^{-2}$, below the lowest shear stress tested in this study i.e. 14.2 dynes $\mathrm{cm}^{-2} .^{11}$ The bacterial response is presumably related to stick-and-roll rolling motion of cells. ${ }^{11,12}$ However, the total displacement of adherent ORN178 cells varied depending on their location on the gradient. In Figure 7.7a the total displacement of ORN178 was measured and compared for cells bound in bins i \& ii, bins iii \& iv and bin $v$. A trend of decreasing ORN178 displacement with increasing surface density was observed, suggesting stronger surface interaction of ORN178 in bin $v$. This is confirmed by grouping ORN178 cells that either moved more than $10 \mu \mathrm{m}$ or less than $10 \mu \mathrm{m}$ in bins i \& ii, bins iii \& iv and bin $v$ (Figure 7.7b). The amount of cells that moved less than $10 \mu \mathrm{m}$ was significantly higher for the region of the highest density of mannose (bin $v$ ) supportive of stronger surface interaction of the bacteria in this region. The proportion of cells that moved a distance of more than $10 \mu \mathrm{m}$ increased with decreasing mannose surface density, highlighting the effect of mannose density on total bacterial displacement in flow conditions. Interesting to note is the non-linear behavior shown in Figure 7.6a, most evident in bins iii \& iv. Here, stabilization of the amount of adhered cells with increasing shear stresses seems to suggest an increased in binding strength between mannose and the bacteria. Since the density of mannose remains unchanged within a region throughout the experiment, the increase in binding strength may presumably be related to so-called catch bonds, force-induced increase of the lifetime of individual receptor-ligand interactions. ${ }^{11}$

a)

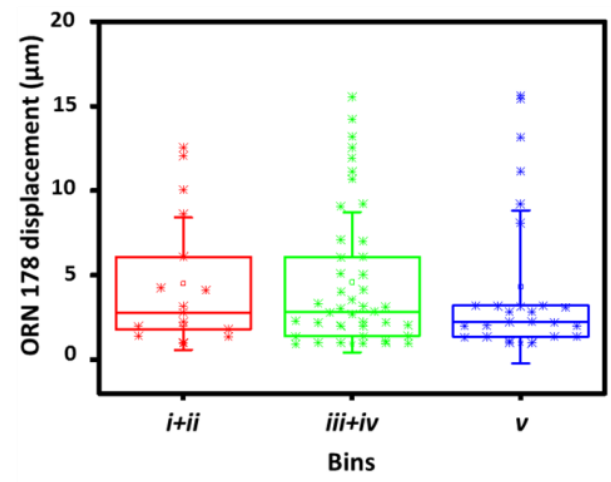

b)

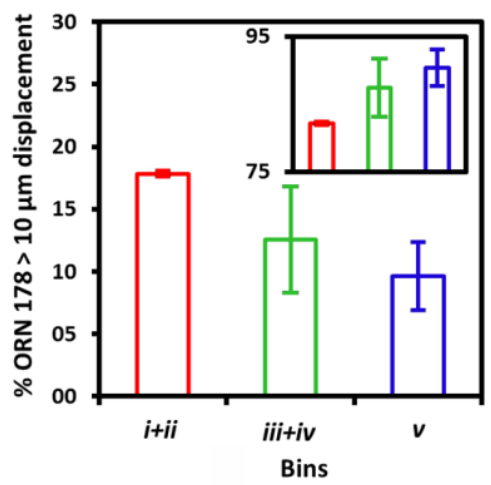

Figure 7.7. Effect of shear stress on ORN178 bound to mannose-gradients. a) Percentage of adherent ORN178 vs. shear stress in bins i-v. b) ORN178 displacement in bins i-ii, iii-iv and v and c) the \% ORN178 cells that moved $>10 \mu \mathrm{m}$ (inset $<10 \mu \mathrm{m}$ ). BLUE bin $v$, GREEN bins iii+iv and RED, bins $i+i i$. 


\subsection{Conclusions}

In conclusion, gradients of mannose were prepared, characterized and used to evaluate the binding of E. coli strain ORN178. FimH binding to the continuous locked-in mannose gradients was specific and shows selective binding above a threshold density of mannose. Moreover, the effect of shear stress on the binding and total displacement of ORN178 cells was investigated. A dependency was found between increasing mannose density, increasing cell adherence and decrease of total cell displacement. Bacteria bound to regions of highest mannose density on the SLB gradient were most prone to retain their cell-surface interactions. Our SLB gradient chip proves to be instrumental for probing the threshold binding in pathogen-lectin interactions on nonfouling SLB surfaces. In a semi-high-throughput fashion and by easily varying the ligand density over a wide range on one chip binding characteristics can be probed under physiologically relevant shear conditions.

\subsection{Experimental Section}

Materials. All starting materials and chemicals were purchased from Sigma-Aldrich, Fluka, Serva, Becton Dickinson, Avanti Polar Lipids, Microchem, Thermo Fisher, Invitrogen and Fujifilm, and they were used as received, unless otherwise stated. Synthesis of mannose-SH is described elsewhere. ${ }^{33}$ Both ORN178 and ORN208 bacterial strain were kindly provided by Prof. Luc Brunsveld, TU/e. MilliQ water with a resistivity higher than $18 \mathrm{M} \Omega \mathrm{cm}^{-1}$ was used in all experiments.

Chip fabrication. A bilayer lift-off recipe was used for fabricating Au electrodes on borofloat glass wafers. First, LOR 5A (MicroChem) was spin-coated, after which normal lithography was performed on top with Olin OiR 907-17 photoresist (FujiFilm) to create a bilayer resist stack. Electrode patterns were made by exposing the photoresist through a patterned photomask and developing in Olin OPD 4262 (FujiFilm). The develop step washed away the exposed photoresist, and etching through the LOR $5 \mathrm{~A}$ layer created an undercut. Then, $5 \mathrm{~nm}$ Ti and $95 \mathrm{~nm}$ Au were deposited via e-beam evaporation (BAK 600, Balzers) The bilayer resist was then removed by sonication in acetone (20 minutes) and isopropanol (10 minutes) followed by 5 minutes immersion in OPD 4262, serving as a sacrificial layer to leave patterned Au electrodes on borofloat glass. To fabricate the $\mathrm{Cr}$ corrals ( $10 \mathrm{~nm}$ thick) in between the Au electrodes, the same procedure was performed a second time, but in this case following alignment with respect to the Au electrodes. 
PDMS flow channel. A silicon flow channel master was produced by standard photolithography steps and deep reactive ion etching. The polydimethylsiloxane (PDMS) flow channels were prepared from a degassed mixture of 10:1 Sylgard 184 elastomer and curing agent (Dow Corning Corp), which was casted onto the silicon master and cured at $80^{\circ} \mathrm{C}$ for 1 hour. The flow channels were cut to size and inlets and outlets were punched using a $1 \mathrm{~mm} \varnothing$ punch (Harris Uni-core, Sigma-Aldrich). After bonding to the chip, a flow channel of 5,000 $\times 500 \times 50 \mu \mathrm{m}$ is prepared.

PDMS bonding. Chips were rinsed and sonicated extensively with acetone, ethanol and MilliQ water, and dried prior to UV-ozone exposure (UV/Ozone Procleaner plus, Bioforce Nanosciences) for at least 20 minutes. After UV exposure, the chips were rinsed with ethanol, water and dried under a stream of nitrogen. Both cut-out PDMS flow channels and cleaned chips were treated with oxygen plasma for 30 seconds at 40 W (Plasma prep II, SPI supplies) after which they were bonded immediately. The chips were placed on a hot plate for 10 minutes at $70^{\circ} \mathrm{C}$ to increase the binding strength. Tygon tubing (VWR, $0.25 \mathrm{~mm}$ inner $\varnothing$ and $0.76 \mathrm{~mm}$ outer $\varnothing$ ) was inserted into the PDMS. The assembled $\mu$ SLB electrophoresis chip was placed in an oven at $60^{\circ} \mathrm{C}$ for 1 hour. Leak-free operation was shown for flow rates up to $2 \mathrm{~mL} / \mathrm{min}$.

Vesicle preparation. 1,2-Dipalmitoyl-sn-glycero-3-phosphocholine (DPPC, Avanti Polar Lipids) was stored as a $10 \mathrm{mg} / \mathrm{mL}$ stock solution in chloroform at $-20^{\circ} \mathrm{C}$. $1,2-$ Dihexadecanoyl-sn-glycero-3-phosphoethanol-amine (DPPE, Avanti Polar Lipids) was stored as a $4 \mathrm{mg} / \mathrm{mL}$ stock in chloroform at $-20^{\circ} \mathrm{C}$. The charged lipid-dye conjugate, Texas Red-1,2-dihexadecanoyl-sn-glycero-3-phosphoethanolamine (TR-DHPE, Invitrogen) was stored as a $1 \mathrm{mg} / \mathrm{mL}$ stock solution in methanol at $-20^{\circ} \mathrm{C}$. Desired molar ratios of the DPPC, DPPE and TR-DHPE were mixed and dried under a flow of nitrogen in a glass vial, and subsequently placed under vacuum overnight. The resulting lipid film was re-suspended by vortexing in MilliQ water to form multilamellar vesicles (MLVs) at $1 \mathrm{mg} / \mathrm{mL}$ at $60^{\circ} \mathrm{C}$. The MLV solution was extruded 11 times through a $100 \mathrm{~nm}$ polycarbonate membrane (Avanti Polar Lipids) at $60^{\circ} \mathrm{C}$. The resulting large unilamellar vesicles (LUVs) were kept at room temperature and used within two weeks.

SLB formation. Prior to SLB formation, wires were soldered onto the gold contact pads and the device was mounted onto a heat plate (IKA RCT classic) using scotch tape. Supported lipid bilayer (SLB) formation was achieved by dilution of the LUV solution to $0.5 \mathrm{mg} / \mathrm{mL}$ in $1 \mathrm{x}$ phosphate-buffered saline (0.01 M PBS pH 7.4, Gibco, lacking $\mathrm{MgCl}_{2}$ and $\mathrm{CaCl}_{2}$ ). Prior to LUV incubation, the chips were flushed briefly with $1 \mathrm{x}$ PBS and heated till $60^{\circ} \mathrm{C}$. Once heated, the chips were incubated with the vesicle suspension for at least 170 
30 minutes to allow for vesicle adsorption and rupture to occur. Subsequently, the chips were washed with MilliQ water $(300 \mu \mathrm{L} / \mathrm{min})$ and allowed to cool down. From this point forth, care was taken to ensure no air bubbles entered the device. Once at room temperature, aqueous $0.5 \mathrm{mM}$ hydroxymethylferrocene $\left(\mathrm{F}_{\mathrm{C}} \mathrm{CH}_{2} \mathrm{OH}\right)$ was flown through the device at the same flow speed.

Mannose coupling. To conjugate mannose to the primary amine of DPPE $5 \mathrm{mM}$ of sulfosuccinimidyl 4-(N-maleimidomethyl)cyclohexane-1-carboxylate (Sulfo-SMCC) in 50 $\mathrm{mM}$ sodium phosphate buffer $(\mathrm{pH} 7.4)$ was allowed to react for 1 hour at room temperature. Subsequently, $1 \mathrm{mM}$ mannose-SH in $50 \mathrm{mM}$ sodium phosphate ( $\mathrm{pH} 6.8$ ) was flown through the device and left to react for 1 hour. Care was taken to prepare fresh solutions in order to prevent NHS hydrolysis and disulfide formation, respectively. Afterwards the chip was extensively rinsed with milliQ prior to $\mu \mathrm{SLB}$ electrophoresis.

$\mu$ SLB electrophoresis. Electric fields were generated and currents were measured with a $\mathrm{CH}$ Instruments $760 \mathrm{D}$ potentiostat with the counter and reference connections shorted. During $\mu S L B$ electrophoresis, a freshly prepared solution of $0.5 \mathrm{mM}$ of $\mathrm{FcCH} 2 \mathrm{OH}$ in MilliQ water was flushed through the chip at $100 \mu \mathrm{L} / \mathrm{min}$. Prior to heating the device a potential difference of $1 \mathrm{~V}$ was applied for at least 30 seconds at room temperature. After that the chip was heated to $60^{\circ} \mathrm{C}$ and the potential difference of $1 \mathrm{~V}$ was applied for 30 minutes. Subsequently, the chip was cooled down rapidly on a heat exchanger for 5 minutes to fix the mannose surface gradient.

Bacterial cell culture. The bacterial strains ORN178 and ORN208 were grown overnight in LB media using tetracycline as the selective antibiotic. These were then spun down at $5,000 \times \mathrm{g}$ for 10 minutes and the supernatant was discarded. The bacteria were washed twice with $10 \mathrm{mM}$ HEPES, $137 \mathrm{mM} \mathrm{NaCl}$, pH 7 buffer by centrifugation and resuspension. Finally the bacteria were reconstituted in this buffer and their optical density at $600 \mathrm{~nm}$ was measured

Bacterial adhesion. OD 0.1 solutions in $10 \mathrm{mM}$ HEPES, $137 \mathrm{mM} \mathrm{NaCl}, \mathrm{pH} 7$ buffer were prepared of ORN178 and ORN208. Prior to flowing the bacteria through the device, $\mathrm{MnCl} 2$ and $\mathrm{CaCl}_{2}$ were added at final concentrations of $1 \mathrm{mM}$ each $200 \mu \mathrm{L}$ of bacterial solution was flown through the chip at $100 \mu \mathrm{L} \mathrm{min}^{-1}$. The flow was stopped and the cells were left to settle for 15 minutes. After the set incubation period, the flow speed was increased incrementally to study the effect of shear stress as well as surface mannose density. 
Fluorescence microscopy. An Olympus inverted IX71 epi-fluorescence research microscope with a Xenon X-cite 120PC as light source and a digital Olympus DR70 camera for image acquisition was used to acquire fluorescence micrographs of the TRDHPE gradient. To this end, green excitation $(510 \leq \lambda \leq 550 \mathrm{~nm}$ ) and red emission ( $\lambda>$ $590 \mathrm{~nm}$ ) was filtered using the U-MWG2 Olympus filter cube. Bacterial adhesion was imaged using to top bright field illumination. To guide the eye, the bacteria were given a false color. In both instances ISO200 camera settings were used to record high quality, low noise images. In case of fluorescence micrographs, care was taken to ensure image acquisition was performed in the linear response regime.

QCM-D. SLBs were formed on borosilicate QCM resonators (Q-Sense). Prior to use, the resonators were cleaned with Piranha for 5 minutes and rinsed with milliQ. After mounting the cleaned resonators in the QCM-D holder. All fluids were exchanged continuously at $100 \mu \mathrm{L} \mathrm{min}^{-1}$ using a peristaltic pump. The temperature was set at $45^{\circ} \mathrm{C}$ allowed to equilibrate while flowing degassed 0.5 times PBS ( $5 \mathrm{mM}$, Sigma-Aldrich, $\mathrm{pH}$ 7.4). After obtaining a stable baseline (less than $\Delta 0.5 \mathrm{~Hz}$ during 10 minutes), $0.5 \mathrm{mg} / \mathrm{mL}$ LUVs were flown through the device. The $1 \mathrm{mg} / \mathrm{mL}$ stock LUV solution in milliQ was diluted 1:1 with 1 times PBS (10 mM, Sigma-Aldrich, pH 7.4) just before vesicle addition. After observing the vesicle fusion process, the tem-perature was lowered to $22^{\circ} \mathrm{C}$ in the presence of the LUV solution. Subsequently, $50 \mathrm{mM}$ sodium phosphate buffer ( $\mathrm{pH} 7.4$ ) was passed through until a stable baseline was reached. A freshly prepared $5 \mathrm{mM}$ sulfoSMCC solution in $50 \mathrm{mM}$ sodium phosphate buffer $(\mathrm{pH} \mathrm{7.4)}$ was allowed to react at room temperature Subsequently, a $1 \mathrm{mM}$ mannose-SH solution in $50 \mathrm{mM}$ sodium phosphate buffer $(\mathrm{pH}$ 6.8) was allowed to react at room temperature. A $1 \mu \mathrm{M}$ Concanavalin A (ConA) solution in $10 \mathrm{mM}$ HEPES, $137 \mathrm{mM} \mathrm{NaCl}, 1 \mathrm{mM} \mathrm{MnCl} 2$ and $\mathrm{CaCl}_{2}$ $\mathrm{pH} 7$ buffer was flown through the device to monitor binding. All QCM-D data is presented from the 5 th overtone.

Data analysis. Image analysis was performed using ImageJ (NIH), Origin (OriginLab) and Excel (Microsoft).

Simulation of shear stress. Finite element analysis (COMSOL 4.4) was performed to gain insights into the shear stress at certain flow speeds in our device. A simulation domain of $500 \mu \mathrm{m} \times 50 \mu \mathrm{m} \times 1 \mathrm{~mm}$ was used to model the flow channel. Na-vier-Stokes equations were solved for incompressible laminar flow (very low Reynolds number) with no-slip boundary condi-tions at the walls. Constant volumetric flow rate was defined at the inlet as laminar in-flow with $2 \mathrm{~mm}$ entrance length. The 3-D model consisted of 430,000 elements to reach mesh independent convergence. For the 172 
simulations, water was taken as the model liquid. A calculated wall shear stress per unit

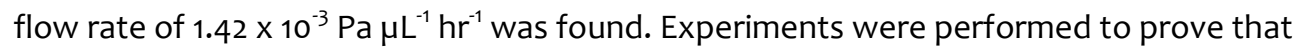
no back pressure is evident at the flow used herein.

Binding plots. Based on the profile plots of the TR-DHPE fluorescence, mol\% per distance was calculated according to literature. ${ }^{34}$ We assumed full conversion of the DPPE to DPPE-mannose. Our QCM-D findings suggest both reactions were complete. Using the calculated mol\% and the average lipid head-group area (DPPC $47.9 \AA^{2}$ ), the amount of mannose per unit area could be calculated. ${ }^{30}$ To study the binding, bins of 20 bacterial cells were studied. The resulting bin area was used to calculate average mannose density per bin.

\subsection{References}

(1) Kiessling, L. L., Gestwicki, J. E., and Strong, L. E. (2000) Synthetic multivalent ligands in the exploration of cell-surface interactions. Curr. Opin. Chem. Biol. 4, 696-703.

(2) Harris, T. J. C., and Tepass, U. (2010) Adherens junctions: from molecules to morphogenesis. Nat. Rev. Mol. Cell Biol. 11, 502-514.

(3) Ottone, C., Krusche, B., Whitby, A., Clements, M., Quadrato, G., Pitulescu, M. E., Adams, R. H., and Parrinello, S. (2014) Direct cell-cell contact with the vascular niche maintains quiescent neural stem cells. Nat. Cell Biol.

(4) Peglion, F., Llense, F., and Etienne-Manneville, S. (2014) Adherens junction treadmilling during collective migration. Nat. Cell Biol. 16, 639-51.

(5) Bischoff, M., and Schnabel, R. (2006) Global cell sorting is mediated by local cell-cell interactions in the C-elegans embryo. Dev. Biol. 294, 432-444.

(6) Zaromytidou, A.-I. (2012) Mechanical control at cell-cell contacts. Nat. Cell Biol. 14, 990-990.

(7) Etienne-Manneville, S. (2008) Polarity proteins in migration and invasion. Oncogene 27, 69706980.

(8) Liang, P. H., Wu, C. Y., Greenberg, W. A., and Wong, C. H. (2008) Glycan arrays: biological and medical applications. Curr. Opin. Chem. Biol. 12, 86-92.

(9) Mrksich, M. (2000) A surface chemistry approach to studying cell adhesion. Chem. Soc. Rev. 29, 267-273.

(10) Nilsson, L. M., Thomas, W. E., Trintchina, E., Vogel, V., and Sokurenko, E. V. (2006) Catch bond-mediated adhesion without a shear threshold - Trimannose versus monomannose interactions with the FimH adhesin of Escherichia coli. J. Biol. Chem. 281, 16656-16663.

(11) Nilsson, L. M., Thomas, W. E., Sokurenko, E. V., and V, V. (2006) Elevated shear stress protects Escherichia coli cells adhering to surfaces via catch bonds from detachment by soluble inhibitors. Appl. Environ. Microbiol. 72, 3005-3010.

(12) Thomas, W. E., Trintchina, E., Forero, M., Vogel, V., and Sokurenko, E. V. (2002) Bacterial adhesion to target cells enhanced by shear force. Cell 109, 913-923.

(13) Pieters, R. J. (2009) Maximising multivalency effects in protein-carbohydrate interactions. Org. Biomol. Chem. 7, 2013-2025. 
(14) Liang, P. H., Wang, S. K., and Wong, C. H. (2007) Quantitative analysis of carbohydrateprotein interactions using glycan microarrays: Determination of surface and solution dissociation constants. J. Am. Chem. Soc. 129, 11177-11184.

(15) Bouckaert, J., Mackenzie, J., de Paz, J. L., Chipwaza, B., Choudhury, D., Zavialov, A., Mannerstedt, K., Anderson, J., Pierard, D., Wyns, L., Seeberger, P. H., Oscarson, S., De Greve, H., and Knight, S. D. (2006) The affinity of the FimH fimbrial adhesin is receptor-driven and quasiindependent of Escherichia coli pathotypes. Mol. Microbiol. 61, 1556-1568.

(16) Voskuhl, J., Sankaran, S., and Jonkheijm, P. (2014) Optical control over bioactive ligands at supramolecular surfaces. Chem. Commun. 50, 15144-15147.

(17) Qi, Z., Bharate, P., Lai, C.-H., Ziem, B., Böttcher, C., Schulz, A., Beckert, F., Hatting, B., Mülhaupt, R., Seeberger, P. H., and Haag, R. (2015) Multivalency at interfaces: Supramolecular carbohydrate-functionalized graphene derivatives for bacterial capture, release, and disinfection. Nano Lett. 15, 6051-6057.

(18) Malinova, V., Nallani, M., Meier, W. P., and Sinner, E. K. (2012) Synthetic biology, inspired by synthetic chemistry. Synth. Biol. 586, 2146-2156.

(19) S. M. Schiller. (2013) Handbook of biofunctional surfaces, chapter 18 (W. Knoll, Ed.). Pan Stanford Publishing Pte. Ltd., Singapore.

(20) Tamm, L. K., and Mcconnell, H. M. (1985) Supported phospholipid-bilayers. Biophys. J. 47, 105-113.

(21) Sackmann, E. (1996) Supported membranes: scientific and practical applications. Science 271, 43-48.

(22) Salaita, K., Nair, P. M., Petit, R. S., Neve, R. M., Das, D., Gray, J. W., and Groves, J. T. (2010) Restriction of receptor movement alters cellular response: physical force sensing by ephaz. Science 327, 1380-1385.

(23) Nair, P. M., Salaita, K., Petit, R. S., and Groves, J. T. (2011) Using patterned supported lipid membranes to investigate the role of receptor organization in intercellular signaling. Nat. Protoc. 6, 523-539.

(24) Krabbenborg, S. O., van Weerd, J., Karperien, M., Jonkheijm, P., and Huskens, J. (2014) Locked-in biomimetic surface gradients that are tunable in size, density and functionalization. ChemPhysChem 15, 3460-5.

(25) Ashley, C. E., Carnes, E. C., Phillips, G. K., Padilla, D., Durfee, P. N., Brown, P. A., Hanna, T. N., Liu, J., Phillips, B., Carter, M. B., Carroll, N. J., Jiang, X., Dunphy, D. R., Willman, C. L., Petsev, D. N., Evans, D. G., Parikh, A. N., Chackerian, B., Wharton, W., Peabody, D. S., and Brinker, C. J. (2011) The targeted delivery of multicomponent cargos to cancer cells by nanoporous particlesupported lipid bilayers. Nat. Mater. 10, 389-97.

(26) Richter, R. P., and Brisson, A. R. (2005) Following the formation of supported lipid bilayers on mica: a study combining AFM, QCM-D, and ellipsometry. Biophys. J. 88, 3422-33.

(27) Hook, F., Kasemo, B., Nylander, T., Fant, C., Sott, K., and Elwing, H. (2001) Variations in coupled water, viscoelastic properties, and film thickness of a Mefp-1 protein film during adsorption and cross-linking: a quartz crystal microbalance with dissipation monitoring, ellipsometry, and surface plasmon resonance study. Anal. Chem. 73, 5796-804.

(28) Dechtrirat, D., Gajovic-Eichelmann, N., Wojcik, F., Hartmann, L., Bier, F. F., and Scheller, F. W. (2014) Electrochemical displacement sensor based on ferrocene boronic acid tracer and immobilized glycan for saccharide binding proteins and E. coli. Biosens. Bioelectron. 58, 1-8.

(29) Groves, J. T., Boxer, S. G., and McConnel, H. M. (1997) Electric field-induced reorganization of two-component supported bilayer membranes. Proc. Natl. Acad. Sci. U. S. A. 94, 13390-13395. 
(30) Korner, A., Deichmann, C., Rossetti, F. F., Kohler, A., Konovalov, O. V., Wedlich, D., and Tanaka, M. (2013) Cell differentiation of pluripotent tissue sheets immobilized on supported membranes displaying cadherin-11. Plos One 8.

(31) Zhu, X. Y., Holtz, B., Wang, Y., Wang, L.-X., Orndorff, P. E., and Guo, A. (2009) Quantitative glycomics from fluidic glycan microarrays. J. Am. Chem. Soc. 131, 13646-13650.

(32) Brown, D. L. (2002) Cardiovascular plaque rupture. Marcel Dekker, New York.

(33) Wendeln, C., Rinnen, S., Schulz, C., Kaufmann, T., Arlinghaus, H. F., and Ravoo, B. J. (2012) Rapid preparation of multifunctional surfaces for orthogonal ligation by microcontact chemistry. Chem. - Eur. J. 18, 5880-5888.

(34) van Weerd, J., Krabbenborg, S. O., Eijkel, J., Karperien, M., Huskens, J., and Jonkheijm, P. (2014) On-chip electrophoresis in supported lipid bilayer membranes achieved using low potentials. J. Am. Chem. Soc. 136, 100-103.

\subsection{Supporting Information}

\subsubsection{Chip with flow-channel design}

a)

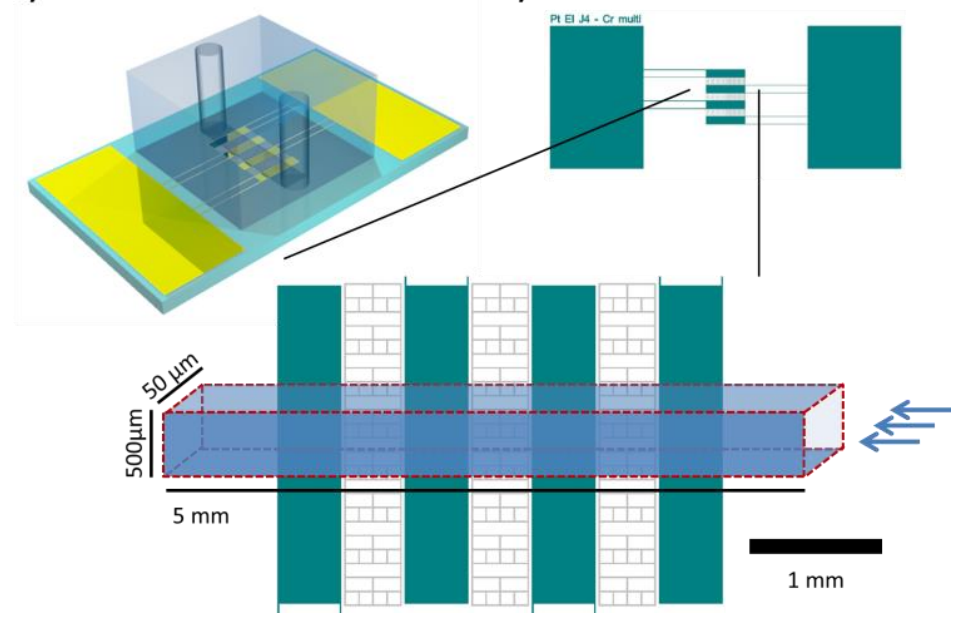

Figure S7.1. a) Schematic of the $\mu S L B$ electrophoresis chip with PDMS flow channel bonded to the surface. b) Chip design showing the different corals and flow channel dimension, scale bar $1 \mathrm{~mm}$. Three different corrals are present on the chip which are $100 \mu \mathrm{m}$ wide and 100, 210 or $430 \mu \mathrm{m}$ long. The PDMS flow channel is bonded to the chip yielding a channel of 5,000 $\times 500 \times 50 \mu \mathrm{m}$ in size. 


\subsubsection{Gradient formation}
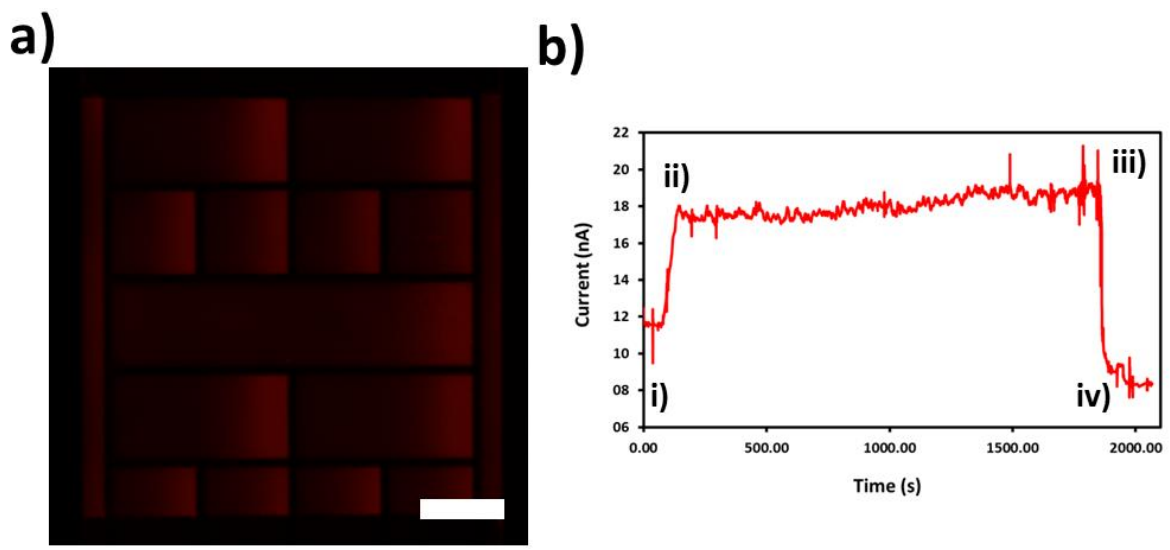

Figure S7.2. Stable SLB gradient formation. a) Fluorescence micrograph of a DPPC-SLB doped with $0.2 \mathrm{~mol} \%$ TR-DHPE $24 \mathrm{hrs}$ after forming the gradient, imaged at room temperature. b) Current measurement during gradient formation, showing the de-pendency between temperature and solution conductivity. Here, four regions can be identified; i SLB at room temperature, ii heating above $\operatorname{Tm}\left(60^{\circ} \mathrm{C}\right)$, iii rapid cooling below Tm to lock-in the gradient and iv the cooled down device. Scale bar $100 \mu \mathrm{m}$.

\subsubsection{ConA specific adhesion control}

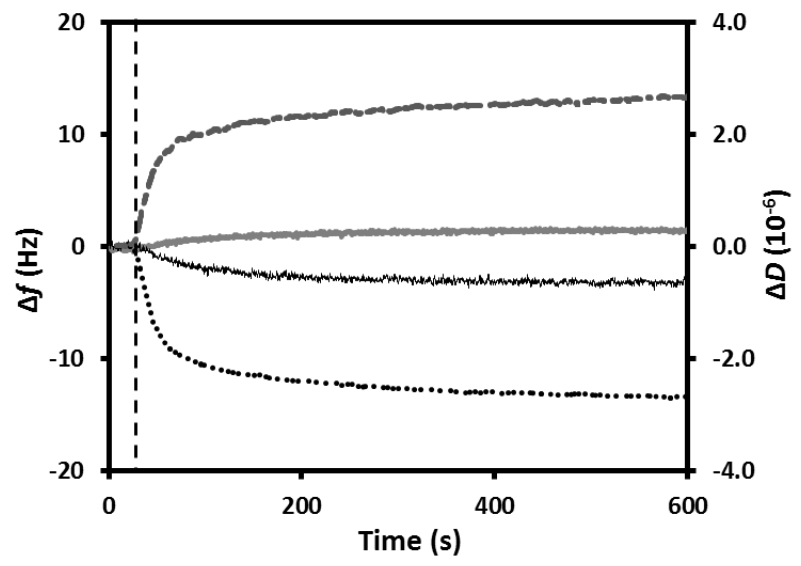

Figure S7.3. QCM-D control experiments of ConA binding on a MPPC-SLB doped with 1 mol\% DPPE and 0,2 mol\% TR-DHPE. Black curves represent $\triangle f$, Grey $\triangle D$. Dotted lines show ConA binding on DPPE-mannose. 


\subsubsection{ORN178 specific adhesion controls}

a)
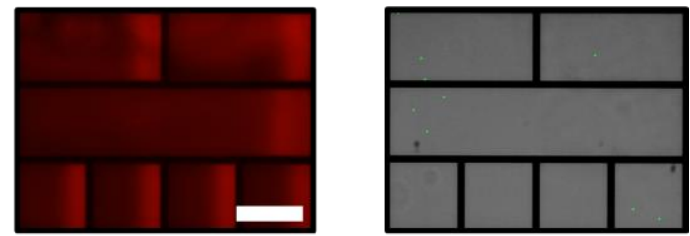

b)

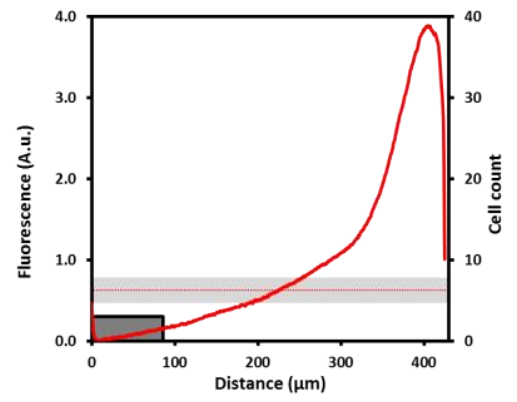

Figure S7.4. Binding of ORN178 to unmodified gradients of DPPE and TR-DHPE. a) Bright field and fluorescence micrographs after ORN178 incubation and flushing at $100 \mu \mathrm{L} \mathrm{min}^{-1}$, scale bar $100 \mu \mathrm{m}$. b) Quantification of cell count vs. TR-DHPE fluorescence for the $430 \mu \mathrm{m}$ long corral. Horizontal red dotted line indicates mean start amount of ORN178 per bin prior to flushing, grey region represents the SD.

\subsubsection{Binding data fits}

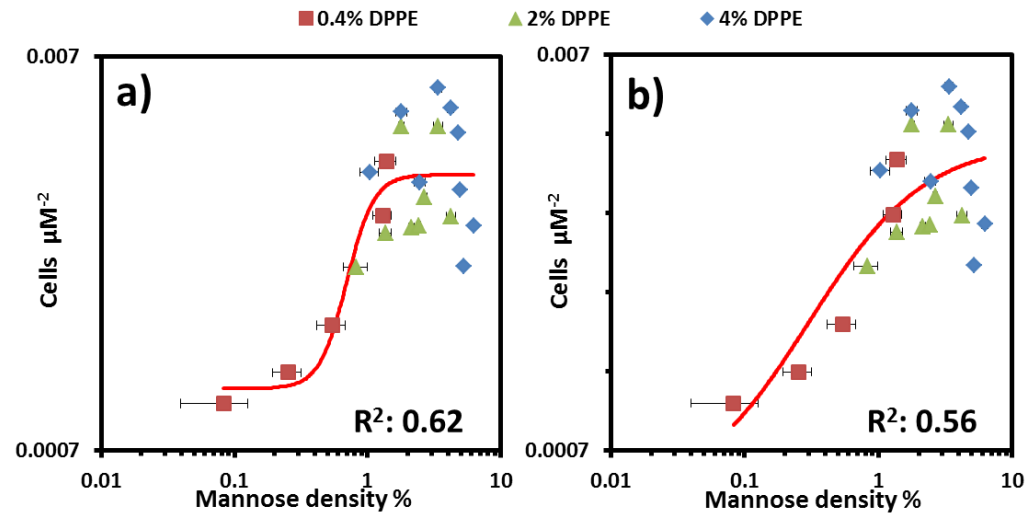


Figure S7.5. Fitting of the binding data from Figure 7.5d with the Hill equation a) leaving the $n$-value variable and $b$ ) fixing $n=1$.

\subsubsection{Shear stress simulations}
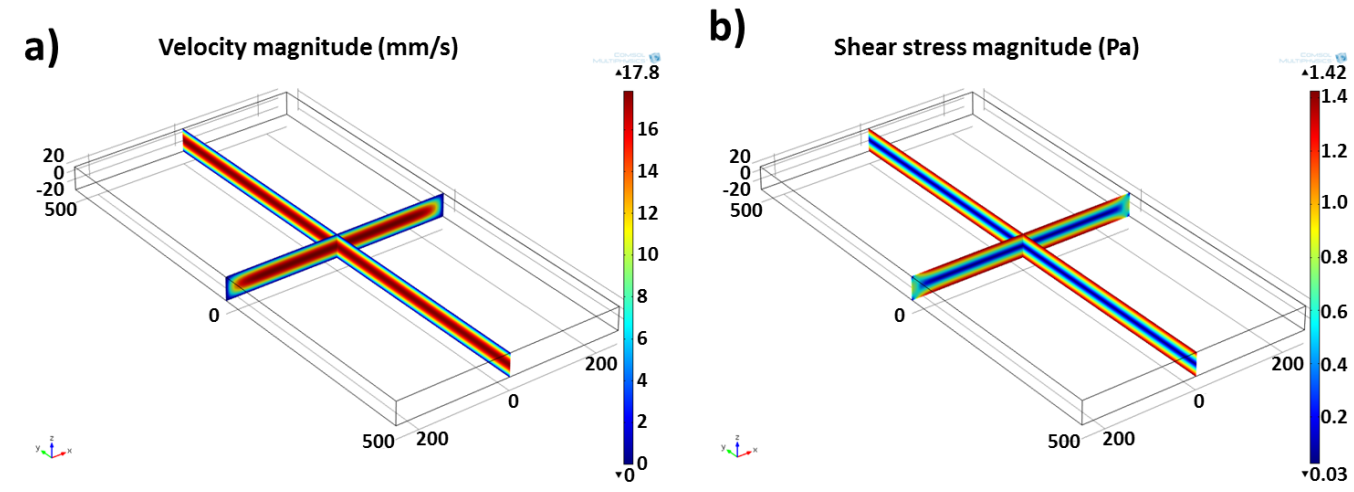

Figure S7.5. Simulation of flow and shear stress in flow channel. a) Longitudinal and axial velocity distributions and b) longitudinal and axial shear stress distributions at $1000 \mu \mathrm{Lr}^{-1}$. 


\section{Chapter 8}

\section{Incorporating bacteria as a living component in supramolecular self-assembled monolayers through dynamic nanoscale interactions}

Supramolecular assemblies, formed through non-covalent interactions, has become particularly attractive to develop dynamic and responsive architectures to address living systems at the nanoscale. Cucurbit[8]uril (CB[8]), a pumpkin shaped macrocylic host molecule, has been successfully used to construct various self-assembled architectures for biomedical applications since it can simultaneously bind two aromatic guest molecules within its cavity. Such architectures can also be designed to respond to external stimuli. Integrating living organisms as an active component into such supramolecular architectures would add a new dimension to the capabilities of such systems. To acheive this, we have incorporated supramolecular functionality at the bacterial surface by genetically modifying a transmembrane protein to display $a C B[8]$-binding motif as part of a cystine-stabilized miniprotein. We were able to confirm that this supramolecular motif on the bacterial surface specifically binds $\mathrm{CB}[8]$ and forms multiple intercellular ternary complexes leading to aggregation of the bacterial solution. We performed various aggregation experiments to understand how $\mathrm{CB}[8]$ interacts with this bacterial strain and also demonstrate that it can be chemically reversed using a competitor. To confirm that this strain can be incorporated with a $\mathrm{CB}[8]$ based architecture, we show that the bacterial cells were able to adhere to $\mathrm{CB}[8] \mathrm{SAMs}$ on gold and still retain considerable motility for several hours, indicating that the system can potentially be used to develop supramolecular bacterial biomotors. The bacterial strain also has the potential to be combined with other $\mathrm{CB}[8]$ based architectures like nanoparticles, vesicles and hydrogels.

The work described in this chapter is part of the article: Sankaran, S., Kiren, M. C., and Jonkheijm, P. (2015) Incorporating bacteria as a living component in supramolecular self-assembled monolayers through dynamic nanoscale interactions. ACS Nano 9, 3579-3586. 


\subsection{Introduction}

In nature, complex cellular processes are almost entirely mediated through dynamic non-covalent interactions between individual molecules or their assemblies. For example, bacteria communicate with their environment through fascinating processes in which natural supramolecular complexes form and disassemble rapidly, allowing for behaviors like chemotaxis, quorum sensing, surface adhesion and biofilm formation. The understanding of such processes has led to the development of powerful synthetic strategies that enable us to dynamically address living cells at the nanoscale. One approach has involved developing self-assembly-based responsive supramolecular materials for bacterial detection, ${ }^{1-3}$ antimicrobial therapies ${ }^{4}$ and even bacterial biomotor systems. ${ }^{5,6}$ An alternative but powerful approach that is still in its infancy involves tailoring supramolecular building blocks directly into the membranes of living cells. For example, Bertozzi and coworkers presented short oligonucleotides on cell surfaces via a metabolic labeling approach to allow for further cellular assembly through DNA hybridization. ${ }^{7}$ Very recently Kros and coworkers reported a liposome fusion process to introduce cholesterol functionalized coiled-coil forming peptides, which allowed for the in vivo decoration of cellular membranes. ${ }^{8}$ In prior work by Yousaf and coworkers such liposome fusion strategies were shown to yield three dimensional tissue-like structures. ${ }^{9}$ These studies not only show us that innovative approaches are required to address cells at the molecular level but also stress that we need to clearly understand the nature of the interactions between our synthetic molecules and complex cellular environments.

In order to perform such studies, supramolecular architectures with mechanical, optical and electrochemical functionalities have been developed through careful design of molecular building blocks. ${ }^{10-13}$ Since these architectures utilize dynamic non-covalent interactions similar to those seen in biological systems, it has become particularly attractive to adopt them for mimicking cellular functions. ${ }^{14}$ For such purposes hostguest systems involving macrocyclic host molecules and aromatic guests have gained a lot of attention since their binding properties are similar to those of proteins. One such group of host molecules, which have been shown to be extremely successful in developing constructs and platforms for biosensing and manipulating living cells are Cucurbit[n]urils $(\mathrm{CB}[\mathrm{n}])$. These are macrocyclic host molecules comprised of methylene-linked glycoluril monomers. They are symmetric and 'barrel'-like in shape with two identical portal regions laced by ureido-carbonyl oxygens. ${ }^{15,16}$ Recently, the microscopic recognition properties of $\mathrm{CB}[\mathrm{n}]$ have been exploited to create protein 
nanowires, ${ }^{17}$ chemical sensors, ${ }^{18}$ molecular machines, ${ }^{19}$ supramolecular materials, ${ }^{20}$ protein chips ${ }^{21}$ and drug delivery systems. ${ }^{22-24}$ In particular, the selective binding of specific amino acid side chains to $C B[\mathrm{n}]$ has been exploited for sensing of protonated and aromatic residues. ${ }^{25-28}$ In contrast to the smaller $\mathrm{CB}[\mathrm{n}]$ homologues, $\mathrm{CB}[8]$ has a larger cavity volume capable of simultaneously accommodating two aromatic amino acids, such as phenylalanine and tryptophan in a $\pi-\pi$ stack geometry to form 2:1 homoternary complexes. ${ }^{29}$ This binding motif has found application in the dimeric and tetrameric assembly of proteins ${ }^{30-32}$ and in hydrogel formation. ${ }^{33}$ On the other hand, multi-responsive bioactive molecular platforms have been developed by using heteroternary complexes involving functional molecules conjugated to e.g. methylviologen, naphthol or azo-benzene. ${ }^{34,35}$ These systems have been used to target proteins and living cells in a manner that can be reversed by applying photochemical and electrochemical stimuli. As the next step, we envisioned that a new dimension of functionality can be introduced to such supramolecular architectures by incorporating living bacterial cells as an active component. This would open up possibilities to endow interesting properties to these dynamic supramolecular architectures like motility, selfrepair, incorporation of engineered proteins etc.

To achieve this, we have developed a novel strategy to introduce specific, dynamic and reversible supramolecular functionality on the bacterial cell surface by adopting a bacterial display system that has been used before exclusively to identify high affinity peptides for various proteins. This technique involves fusing a cystine-stabilized miniprotein bearing randomized peptide sequences to the $\mathrm{N}$-terminus of a modified transmembrane protein (enhanced Circularly Permutated outer membrane protein $\mathrm{X}$ eCPX). ${ }^{36,37}$ Using this powerful technique to render chemical functionality to the bacterial surface has not yet been explored. Accordingly, we show that a bacterial strain with supramolecular functionality can be developed by displaying a miniprotein that can reversibly bind with the supramolecular host molecule cucurbit[8]uril (CB[8]). Peptides containing tryptophan or phenylalanine like FGG, WGG, GGFGG and GGWGG have been shown to bind $\mathrm{CB}[8]$ with dissociation constants in the low $\mu M$ range. ${ }^{38,39}$ For our supramolecularly addressable bacterial strain, we grafted the GGWGC sequence into one loop of a cystine stabilized miniprotein named Min-23 (1), then genetically fused this to the N-terminal of eCPX and expressed it in an MC1061 strain of E.coli. Since bacterial expression systems produce multiple copies of the cloned protein, we postulated that multivalent interactions could be established between bacterial cells in the presence of $\mathrm{CB}[8]$, resulting in their aggregation. Few reports exist on the use of multivalent host-guest interactions for macroscopic self-assembly, e.g. 
the assembly of cyclodextrin-functionalized and guest-functionalized hydrogels and adhesion of CB-functionalized silicon chips with guest-functionalized counterparts. ${ }^{40,41}$ However, in living systems like bacteria, various voluminous cell surface components like flagellae and pili could prevent $\mathrm{CB}[8]$ from accessing miniproteins on two separate bacterial cell membranes. Also, $\mathrm{CB}[8]$ could probably bind only adjacent miniprotein guests on the same bacterial surface, preventing aggregation and self-assembly onto monolayers. Finally, unspecific interactions between $\mathrm{CB}[8]$ and aromatic amino acids on other transmembrane proteins could possibly reduce the specificity of the system. Despite these potential hurdles, we were able to clearly show that specific inter-cellular supramolecular interactions are established by ternary complex formation between $\mathrm{CB}[8]$ and two miniprotein guests, causing bacterial assembly in solution and surfaces. We used this phenomenon to study the characteristics of the interactions between $\mathrm{CB}[8]$ and our bacterial strain. Once we gained a clear understanding of the interaction, we tested the assembly of this bacterial surface on $\mathrm{CB}[8]$ modified SAMs. We attained specific and reversible adhesion of our bacterial cells on these surfaces and saw that the cells even retained their motility indicating that the non-covalent interactions allow the cells to remain active.

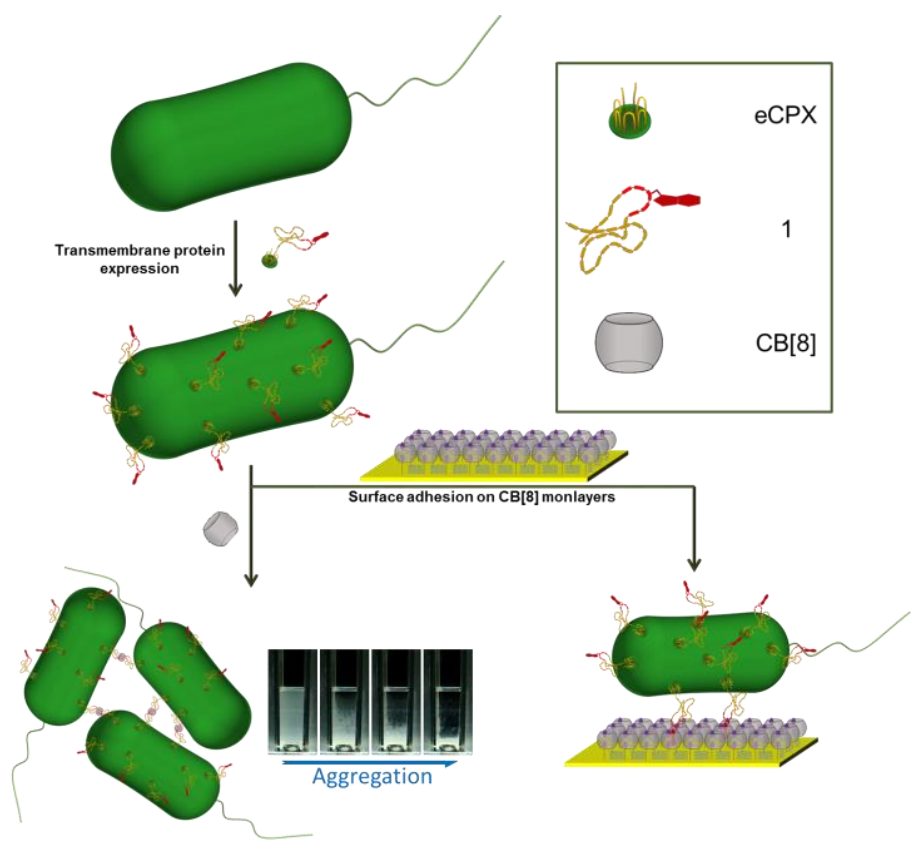

Figure 8.1. Development of a $\mathrm{CB}[8]$-addressable bacterial strain. Min-23 construct 1 contains peptide sequence GGWGG in one loop. This was genetically fused to a modified outer membrane protein eCPX, which enabled it to be displayed on the outer membrane and bind $C B$ [8]. 


\subsection{Results and Discussions}

\subsubsection{Detection of the display proteins by SDS-PAGE}

First we wanted to test whether our modified miniproteins were displayed on the surface of our bacterial cells. As negative control in all experiments, a $\beta$-trypsin inhibitor knottin (2) ${ }^{42}$ was separately expressed and fused to the $\mathrm{N}$-terminal of eCPX. To verify that the display proteins were being expressed, SDS-PAGE was performed on cell lysates. Due to their low expression yields and poor solubility, a modified strategy was used. To maximize production, the cultures were allowed to express the proteins for 16 hrs after arabinose induction. Lysis was performed using Bugbuster ${ }^{\circledR}$ and benzonase and to improve the solubility of these outer membrane proteins, an equal volume of $5 \%$ (w/v) SDS and $6 \mathrm{M}$ Guanidine $\mathrm{HCl}$ was also added during the lysis. These samples were then mixed with an excess (3 volumes) of sample loading buffer (125 mM Tris- $\mathrm{HCl}, 20 \%$ $\mathrm{v} / \mathrm{v}$ glycerol, $5 \%$ SDS, $0.02 \%$ bromophenol blue, $10 \% \mathrm{v} / \mathrm{v} \quad \beta$-mercaptoethanol) and heated to $99^{\circ} \mathrm{C}$ for 15 mins. These were then resolved using a $12 \%$ polyacrylamide gel. Lysates from MC1061 cells displaying 1, 2 and nothing were compared. In Figure 8.2a, the bands in the boxes correspond to the molecular weights of the appropriate proteins for each sample. To make sure that the bands did indeed correspond to our outer membrane proteins, we performed another experiment where we lysed the cells using Bugbuster ${ }^{\circledR}$ and benzonase and spun down to separate the soluble and insoluble fraction. The insoluble fraction, in the form of a pellet, was resolubilized in a solution of $6 \mathrm{M}$ Guanidine $\mathrm{HCl}$ containing $5 \%(\mathrm{w} / \mathrm{v}$ ) SDS. Both fractions were separately mixed with 3 volumes of sample loading buffer and heated to $99^{\circ} \mathrm{C}$ for 15 minutes. It can be seen in Figure $8.2 \mathrm{~b}$ that the bands from the previous gel got separated between the soluble and insoluble fractions. As expected, the bands corresponding to our outer membrane display proteins were seen in the insoluble fraction. 


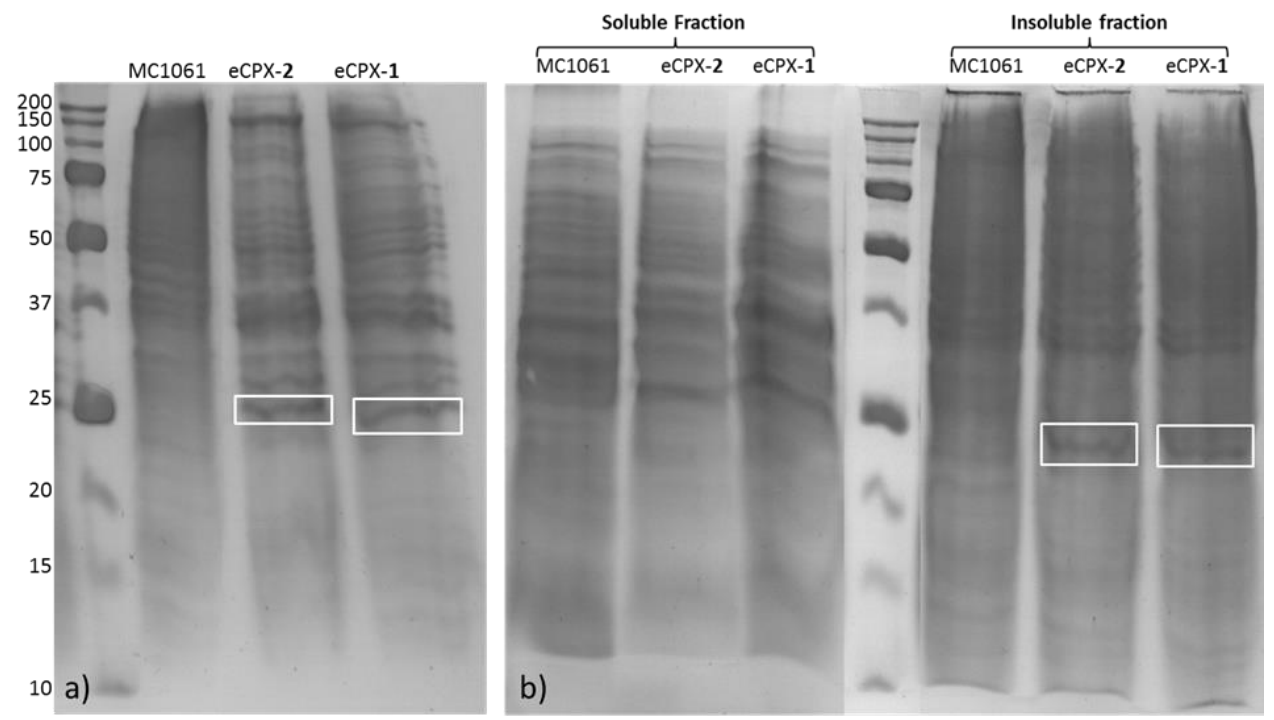

Figure 8.2. SDS-PAGE results of resolving solubilized cell lysate proteins from MC1061 cells displaying nothing, 1 and 2. The bands in the squares correspond to the required proteins. The numbers on the left correspond to the molecular weight of each band of the ladder Precision (Plus Protein ${ }^{\text {TM }}$ Unstained Standards) in kDa. eCPX-1 and eCPX-2 have a molecular weight of $\sim 23$ kDa. a) Entire cell lysate solubilized in guanidine $\mathrm{HCl}$ with SDS and b) cell lysates divided into soluble and insoluble fractions.

\subsubsection{Testing the display system}

Flow cytometry experiments with fluorescein isothiocyanate (FITC)-labeled trypsin were carried out as an additional verification that the miniproteins were being displayed (Figure 8.3). Bovine $\beta$-trypsin was labeled using fluorescein isothiocyanate (FITC) with nearly $40 \%$ efficiency as determined from its UV absorbance spectra. Bacterial cells displaying 2 in PBS were incubated with $50 \mathrm{nM}$ of this labeled protein for $1 \mathrm{hr}$ at $4^{\circ} \mathrm{C}$. The cells were spun down at $6000 \mathrm{~g}$ for 5 mins, the supernatant was discarded and the cells were resuspended in PBS. This was repeated twice to wash the cells thoroughly. As a negative control, a bacterial strain displaying a different miniprotein was used.

After calibration of the flow cytometer Hoechst dye 33342 stained bacterial cells to be counted were flowed through the machine. Among bacterial cells displaying 2, nearly $40 \%$ were detected to be fluorescent, indicating that the labeled trypsin was bound to 
them as shown in Figure 8.3. In the case of the negative control only a negligible proportion was detected as fluorescent.

Bacteria displaying 2

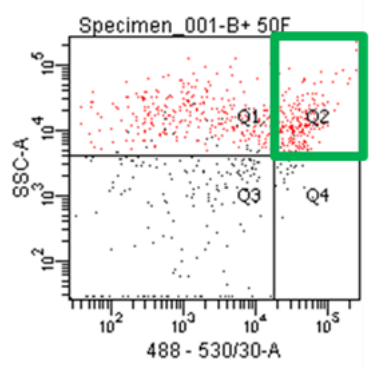

Negative Control

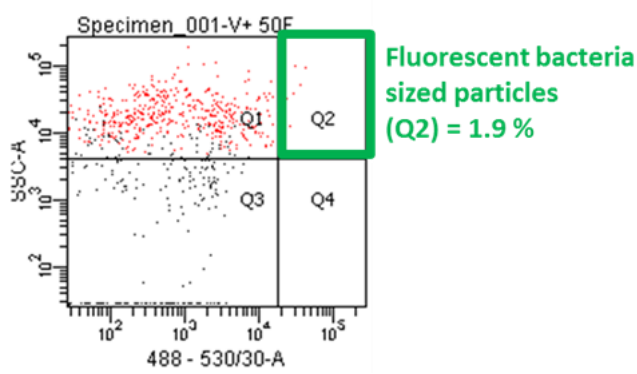

Figure 8.3. Flow cytometry results of labeled trypsin binding display bacteria. The red dots indicate bacteria sized particles and quadrant Q2 represents the fraction of bacteria which are detected as fluorescent.

\subsection{3. $C B[8]$-mediated bacterial aggregation}

In the case of the strain engineered to display our GGWGG peptide sequencecontaining miniprotein $\mathbf{1}$, we expected that $\mathrm{CB}[8]$ could cause aggregation of a bacterial culture if the miniproteins were sufficiently accessible. To test whether such supramolecular assembly would occur despite the hurdles mentioned before, we prepared solutions containing $25 \mu \mathrm{M} \mathrm{CB}[8]$ and the bacteria displaying 1 and 2 . Within 20 min aggregation was visible to the naked eye in culture displaying $\mathbf{1}$ and was recorded as shown in Figure 8.4 (Section 8.7, Video 8.1). In the next $10 \mathrm{~min}$, nearly all the bacteria had been pulled down. In the culture displaying 2, some aggregation was seen around 50 min, but appeared gradual and led to less than a third of the culture being pulled down over a total time of $90 \mathrm{~min}$. These results were also recorded by measuring the optical density of the cultures at $600 \mathrm{~nm}$ (Figure $8.4 \mathrm{~b}$, inset). To enlarge the probing volume and simultaneously record multiple samples we opted to quantify the data from the recorded videos by taking the mean grey value of the cultures as a measure of the bacterial density while monitoring at constant light intensity (Figure 8.4b and Section 8.7 , Video 8.1). The specificity of the assembly was always verified using the bacterial culture displaying 2 as a negative control while performing all the ensuing experiments. Staining the cells with Hoechst dye 33342 enabled us to clearly see that over large areas the bacterial cells displaying 2 are distributed quite homogeneously (Figure 8.4d) while those displaying 1 are aggregated into clusters (Figure 8.4e). To further verify that aggregation is caused specifically by ternary complex formation we 
used $\mathrm{CB}[7]$ as a negative control as the smaller cavity size of $\mathrm{CB}$ [7] can host only one tryptophan at a time. As expected, aggregation rapidly occurred only in the culture mixed with $25 \mu \mathrm{M} \mathrm{CB}[8]$ while no aggregation was witnessed in the culture mixed with $25 \mu \mathrm{M} C B[7]$ even after a much longer time (Figure 8.4C and Section 8.7, Video 8.2).
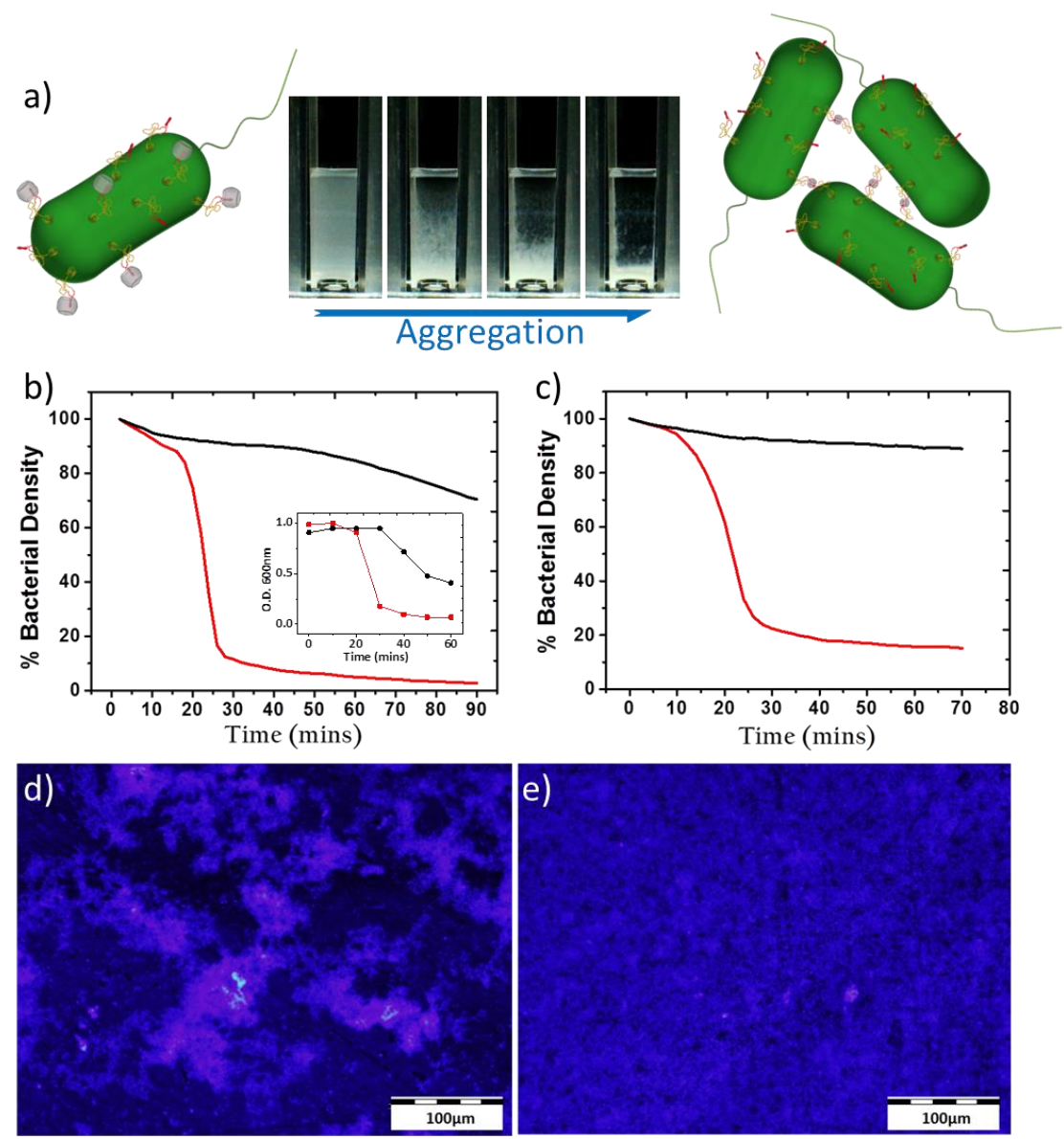

Figure 8.4. (a) Snapshot images of the aggregation of bacteria in solution upon addition of $C B[8]$. Plots displaying aggregation kinetics, obtained from time-lapse snapshot, using b) 1.0 0.D.60onm bacterial solutions displaying 1 (red) and $\mathbf{2}$ (black) with $25 \mu M C B$ [8]. Inset represents data obtained from following optical density at $600 \mathrm{~nm}$. c) 1.0 O.D.600 nm bacterial solutions displaying 1 with 25 $\mu M C B[8]$ (red) and $C B$ [7] (black). Epifluorescence microscopy images of $\mathrm{H} 33342$ stained bacteria forming clusters when mixed with $\mathrm{CB}[8]$ in the case of cells displaying $1(d)$ and absence of clusters seen in the case of cells displaying $2(e)$. 


\subsubsection{Aggregation kinetics}

Confident that the assembly occurred due to specific intercellular interactions between $\mathrm{CB}[8]$ and our surface-displayed miniproteins, we decided to study some characteristics of this supramolecular phenomenon. Accordingly, we performed aggregation experiments with different $\mathrm{CB}[8]$ concentrations and found that the rate was clearly dependent on this parameter (Figure 8.5a and Section 8.7, Video 8.3). Plotting the aggregation rates against $\mathrm{CB}[8]$ concentrations gave us a binding-curve (Figure $8.5 \mathrm{~d}$ ) from which we derived an EC50 value of around $7 \mu \mathrm{M}$ using the 4-parameter logistic equation:

$$
R=d+\frac{(a-d)}{1+\left(\frac{x}{E C 50}\right)^{b}}
$$

where ' $R$ ' represents response values, which in our case corresponds to the rates of aggregation, ' $a$ ' and ' $d$ ' are the lower and upper asymptote values respectively, ' $E C_{50}$ ' is the inflection point, which is equivalent to the concentration corresponding to the half maximal rate of aggregation and ' $b$ ' is the slope at the inflection point.

As expected, this is within an agreeable range for the binding of $\mathrm{CB}[8]$ with such aromatic amino acid containing peptides. Depending on the peptide sequence and the $\mathrm{N} / \mathrm{C}$ terminal nature of the aromatic amino acid, it has been determined that $\mathrm{CB}[8]$ binds with dissociation constants ranging from $10^{-6}$ to $10^{-3} \mathrm{M}$ as shown in Table 8.1 
Table 8.1. Reported binding affinities of various aromatic amino acid based peptides with $\mathrm{CB}$ [8]. ${ }^{43-45}$ All binding affinities reported are for $\mathrm{CB}[8]$ binding with the underlined aromatic amino acids in the peptide sequences. $K_{d M v}$ is the dissociation constant for an amino acid $(X)$ binding a preformed $\mathrm{CB}[8]$-Methylviologen binary complex. $\boldsymbol{K}_{\mathbf{d} 1}$ is the dissociation constant for an amino acid $(X)$ binding free $C B[8] . K_{d 2}$ is the a dissociation constant for an amino acid $(X)$ binding a preformed $C B[8]-X$ binary complex. $\boldsymbol{K}_{\text {dter }}$ is the overall dissociation constant of a $\mathrm{CB}[8]: \mathrm{X}_{2}$ ternary complex

\begin{tabular}{|c|c|c|c|c|}
\hline $\mathrm{X}$ & $\begin{array}{l}M V: C B[8]: X \\
\left(K_{d M V}\right)(M)\end{array}$ & $\begin{array}{l}X: C B[8] \\
\left(K_{d 1}\right)(M)\end{array}$ & $\begin{array}{l}X: C B[8]: X \\
\left(K_{d 2}\right)(M)\end{array}$ & $\begin{array}{l}C B[8]: X 2 \\
\left(K_{d t e r}\right)\left(M^{2}\right)\end{array}$ \\
\hline Tryptophan ( $\underline{\mathbf{W}})$ & $23 \times 10^{-6}$ & - & - & $15 \times 10^{-9}$ \\
\hline Phenylalanine (E) & $188 \times 10^{-6}$ & - & - & $9.1 \times 10^{-9}$ \\
\hline Tyrosine $(\underline{Y})$ & $4.55 \times 10^{-4}$ & - & - & - \\
\hline WGG & $7.7 \times 10^{-6}$ & $7.7 \times 10^{-6}$ & $35.7 \times 10^{-6}$ & $0.3 \times 10^{-9}$ \\
\hline GWG & $4.76 \times 10^{-5}$ & - & - & - \\
\hline GGW & $3.23 \times 10^{-4}$ & - & - & - \\
\hline FGG & - & - & - & $6.7 \times 10^{-12}$ \\
\hline GGWGG & $40 \times 10^{-6}$ & - & - & - \\
\hline AEFRH & - & $1.32 \times 10^{-6}$ & $2.10 \times 10^{-5}$ & $2.67 \times 10^{-11}$ \\
\hline LVFIA & - & $7.99 \times 10^{-6}$ & $1.63 \times 10^{-5}$ & $1.30 \times 10^{-10}$ \\
\hline VIFAE & - & $2.68 \times 10^{-8}$ & $2.36 \times 10^{-6}$ & $6.33 \times 10^{-14}$ \\
\hline
\end{tabular}

Subsequently, we also performed aggregation experiments keeping $C B[8]$ concentration at $25 \mu \mathrm{M}$ and varying the bacterial densities. The rate of aggregation increased with higher bacterial densities as seen in Figure 8.5b and Video 8.4 (Section 8.7). To determine the order of the aggregation event, we took bacterial density as concentration and the reciprocal of aggregation rate as time. A plot of In(Bacterial density) against reciprocal of aggregation rate resulted in a linear trend, appropriately 
indicating that the complex phenomenon of aggregation follows first order kinetics as a function of bacterial density (Figure 8.5e). This is most likely due to the fact that the concentration of the surface-displayed miniproteins amounts to low $\mathrm{nM}$ values at the bacterial densities we use. Thus there is a huge excess of $\mathrm{CB}[8]$ available in solution and its concentration does not vary significantly even when all miniproteins are bound to $\mathrm{CB}[8]$ molecules. This amount of $\mathrm{CB}[8]$ is required to satisfy the affinity requirements for ternary complex formation as determined in Figure $8.5 \mathrm{~d}$ but also causes the phenomenon to follow first order kinetics with dependence on bacterial density.

From these experiments, we were able to postulate a simplified mechanism for the aggregation (Figure 8.5g), similar to some that have been proposed to explain aggregation of lipid vesicles. ${ }^{46}$ In this model, we propose that a first quick step occurs where $C B[8]$ binds to the GGWGG motif on the bacterial surface since $C B[8]$ concentration is several orders higher than the GGWGG motif (low nM range). Thus the coverage of $\mathrm{CB}[8]$ on the bacterial surface varies with $\mathrm{CB}[8]$ concentration. This converts the bacterial cells into active binding entities that are able to stick to each other on collision. First clusters form and grow in size leading to aggregation. Since this step depends on the number of collisions that occur over time, varying the bacterial density has a direct influence on the rate of aggregation.

The ability to reverse the effects of supramolecular interactions is a powerful property of such systems. Agitation of an aggregated sample disperses the bacteria in solution as smaller clusters but aggregation reoccurs when the agitation is stopped. To reverse the aggregation effect we selected a peptide with an $\mathrm{N}$-terminal phenylalanine followed by 6 glycines (FG6) as a $\mathrm{CB}[8]$ competitor. We first performed cell viability tests, which ensured that FG6 was not cytotoxic (Figure 8.7, Experimental section). To study the inhibition of aggregation, we initially allowed bacterial solutions to aggregate in the presence of $25 \mu \mathrm{M} \mathrm{CB}$ [8]. Different concentrations of FG6 up to $1 \mathrm{mM}$ were then added to each sample and briefly agitated by mild shaking. The agitation was then stopped and re-aggregation was monitored. The sample with no added FG6 aggregated rapidly, whereas those in which FG6 was added aggregated at rates depending on the FG6 concentration (Figure 8.5C and Section 8.7, Video 8.5). The degree to which the rate of aggregation reduced in the presence of FG6 was taken as a measure of the extent of inhibition. This, calculated as percentage, when plotted against FG6 concentration, provided a trend, from which we were able to derive an inhibition constant (IC50) of $46 \mu \mathrm{M}$ using equation 8.1 (Figure 8.5f), indicating that the addition of a soluble competitive guest peptide inhibits the association of bacteria by blocking all of the available $\mathrm{CB}[8]$ hosts. 
a)
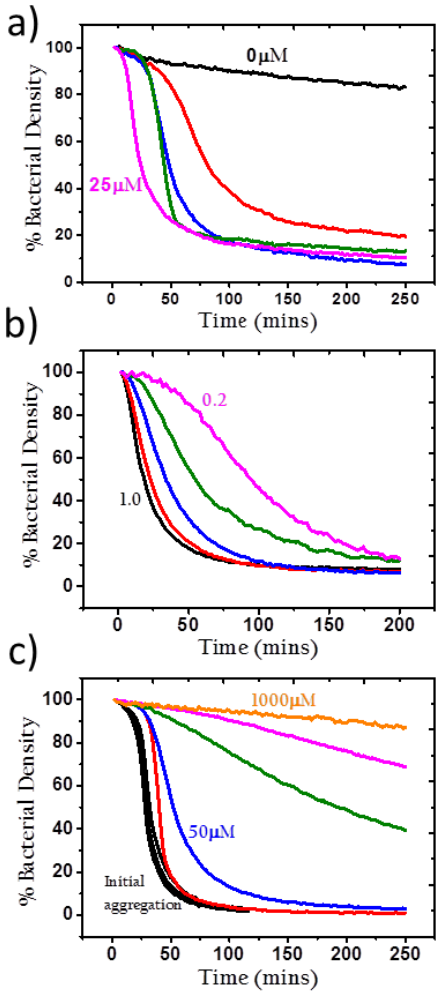

d)

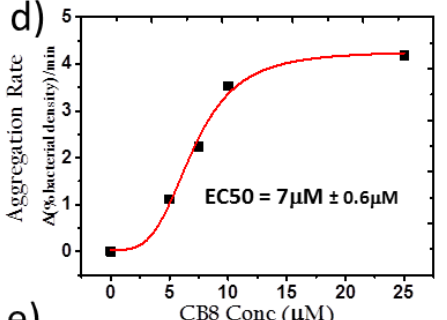

e)

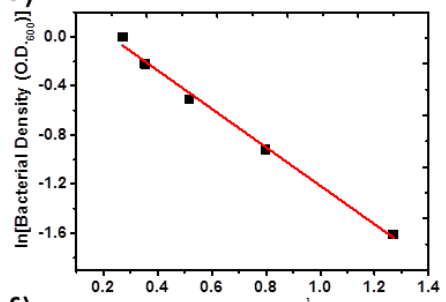

f)

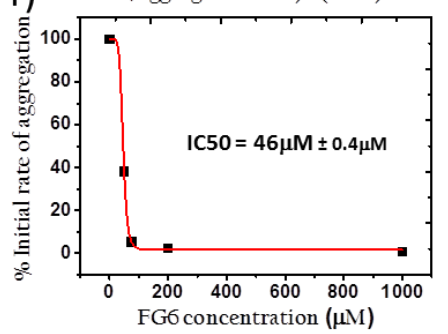

g)
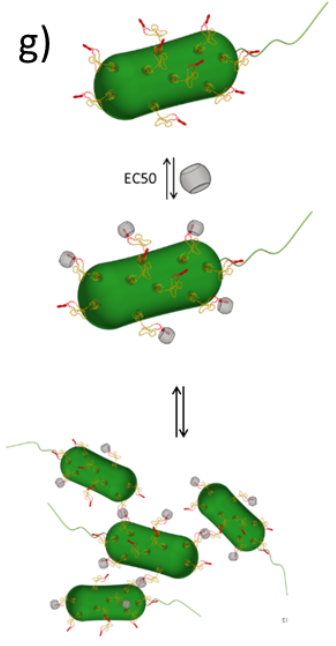

IC50
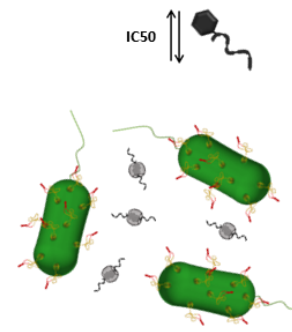

Figure 8.5. (a) Aggregation of 1 O.D.60onm bacterial cultures displaying 1 when mixed with $0 \mu M$ (black), $5 \mu M$ (red), $7.5 \mu M$ (blue), $10 \mu M$ (pink), $25 \mu M$ (green) $C B[8] . b)$ Aggregation occurring when $25 \mu \mathrm{M} \mathrm{CB}[8]$ is mixed with bacterial cultures displaying 1 having O.D.6oonm values of 1 (black), 0.8 (red), 0.6 (blue), 0.4 (green) and 0.2 (pink).c) Initial aggregation of individual samples all containing $25 \mu M C B[8]$ and 1.0 O.D.60onm density of bacterial cultures displaying 1 (black lines). The colored curves represent aggregation occurring after adding o $\mu M$ (red), $50 \mu \mathrm{M}$ (blue), $75 \mu \mathrm{M}$ (green), 200 $\mu M$ (pink) and $1000 \mu M$ (orange) FG6 peptide to the samples and briefly agitating them. d) Aggregation rates vs $\mathrm{CB}$ [8] concentration fitted with equation 8.1 to obtain an EC50 value. e) In(Bacterial density) Vs reciprocal of aggregation rate with a linear fit indicating first order kinetics. f) Reduction in the rate of aggregation plotted as a \% of initial aggregation against different concentrations of FG6 fitted to equation 8.1. g) Proposed mechanism of aggregation and reversibility with each step of the process next to the plots analyzing the corresponding kinetics. 


\subsubsection{Supramolecular surface adhesion}

Having confirmed the surpramolecular nature of the interaction between the bacterial surface and $\mathrm{CB}[8]$ and determined its various characteristics, we intended to show that the bacterial strain could be incorporated into an existing molecular architecture of $\mathrm{CB}[8]$. We had previously shown that it was possible to use self-assembled monolayers (SAMs) of $\mathrm{CB}[8]$, non-covalently displaying an RGD motif, for reversible cell adhesion. ${ }^{47}$ Incorporating our bacterial strain into these SAMs would allow the possibility to introduce interesting properties to the architecture. It has been shown in the past that by adhering bacterial cells to surfaces, their motility can be used to convert chemical energy into mechanical energy for micron scale devices. ${ }^{5,6}$ We thought our bacterial cells on $\mathrm{CB}[8]$-bearing surfaces would still retain a certain extent of motility due to the non-covalent nature of the adhesion. Also, in this system, we avoid chemical modification of the bacterial surface, which is usually prone to alter properties of cells.

To test this out, we first attempted to immobilize our bacteria onto $\mathrm{CB}[8]$ monolayers. The monolayers were prepared on gold using a strategy to insert $C B[8]$-methylviologen thiol inclusion complexes into a repellent monolayer as described previously by us. On incubation of such substrates with bacteria displaying $\mathbf{1}$ and $\mathbf{2}$ we observed that the bacterial cells displaying $\mathbf{1}$ adhered in much greater numbers than the cells displaying $\mathbf{2}$ (Figure 8.6a). Using atomic force microscopy (AFM) we were able to obtain higher resolution images of the immobilized bacterial cells with their flagellae seen as thin twisted fibers (Figure 8.6b).

Using a flow cell, we allowed bacterial cells displaying 1 to adhere to $\mathrm{CB}[8]$ monolayers and flushed with buffer to remove non-specifically adhered bacteria. The specifically surface bound bacteria were highly motile, tracing long paths with considerable speed (Section 8.7, Video S6). Surprisingly, the rate of motility that we observed was much higher than other techniques found in literature. ${ }^{5}$ This movement at the surface was seen for up to 4 hours without alteration even when the flow cell was turned upside down or a flow of $1 \mathrm{ml} / \mathrm{min}$ was employed. The rate of motility seems to follow a Gaussian distribution with a peak close to $20 \mu \mathrm{m} / \mathrm{s}$ (Figure 8.6c). In comparison, motile E. coli cells in solution are known to reach speeds of $20-40 \mu \mathrm{m} / \mathrm{s}$ indicating that supramolecular surface immobilization barely hinders this motility. To test the reversibility of this binding, we used the $\mathrm{CB}[8]$-binding peptide $\mathrm{FG6}$. As expected, most of the bacteria rapidly detached from the surface. Figure $8.6 \mathrm{~d}$ indicates that within the first $10 \mathrm{~min}$ a majority of the cells detach and at 60 min very few isolated cells are still 
seen at the surface. The detachment follows first order kinetics with an observed first order dissociation rate constant of about $0.13 \mathrm{~min}^{-1}$.
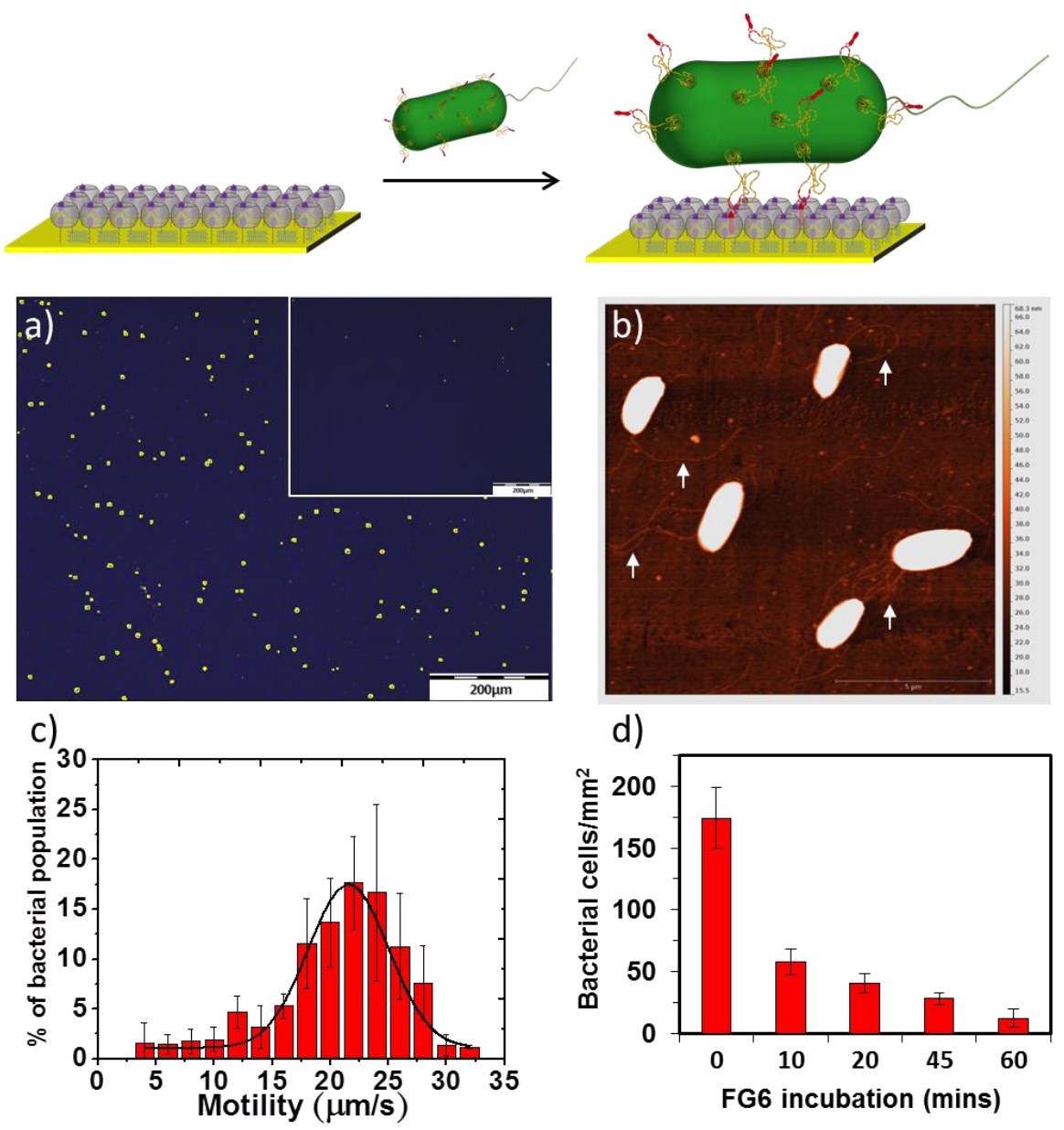

Figure 8.6. Bacterial surface immobilization. a) Bacterial cells displaying 1 immobilized in significant numbers compared to bacterial cells displaying 2 (inset). Bacterial cells have been provided false colors to improve their visibility. b) E.coli cells and their flagellae (indicated by the arrows) seen using high resolution AFM. c) Distribution of the motility of immobilized bacteria fitted with the Gaussian function. Average rate of motility and deviation values were determined from 4 individual videos spanning $50 \mathrm{~s}$ with approximately 30 motile bacterial cells in each. d) Bacterial cells $/ \mathrm{mm}^{2}$ remaining on the surface vs amount of time the surface was incubated with FG6. Bacterial counts and deviations were obtained from 4 separate representative images. 


\subsection{Conclusion}

In conclusion, we have successfully developed a supramolecularly accessible bacterial strain that can be used in conjunction with supramolecular architectures in solution and surfaces. The current study focuses on specificity towards $C B$ [8] but the techniques presented can also be used to develop and study other strains for different host molecules. It would even be possible to recombinantly transfer this modified transmembrane protein to other bacterial strains like Shigella and Salmonella, which also express the outer membrane protein X. Currently we are modulating the characteristics of the surface based interactions and looking into applying this bacterial strain with other supramolecular architectures. Furthermore, we demonstrate that the bacterial cells were able to adhere to $\mathrm{CB}[8]$ monolayers and still retain considerable motility for several hours indicating that the system can be used to develop supramolecular bacterial biomotors, which hold the promise to become a macroscopic alternative to molecular motors. ${ }^{48}$

\subsection{Acknowledgements}

We would like to thank Shyam Sankaran for his contribution towards the image processing, Jasper van Weerd for his help in recording the AFM images and Emanuela Cavatorta for providing the FG6 peptide. We would also like to thank Dr. Jennifer Getz and Prof. Patrick Daugherty from the University of California, Santa Barbara for providing a very clear procedure on how to establish a bacterial display system and their prompt and helpful replies to several questions regarding the same.

\subsection{Experimental Section}

Cloning of E.coli expression plasmids. Plasmid pB33еCPX was a gift from Patrick Daugherty (Addgene plasmid \# 23336). It contained the gene of the enhanced circularly permutated outer membrane protein $X(e C P X)$. The DNA sequences of 1 and $\mathbf{2}$ were purchased from Eurofins MWG Operon. These sequences were ligated between OmpX signal sequence and eCPX using BsrGl and Nhel restriction sites, which were introduced as follows. OmpX was amplified with a BsrGl site at its 3'end and eCPX was amplified with a Nhel site at its 5'end. 1 and 2 were amplified with BsrGl and Nhel restriction sites at their 5'and 3'ends. These three amplified constructs and the plasmid were digested with appropriate restriction enzymes, purchased from NEB, and ligated together in one reaction. These ligated products were transformed into NovaBlue competent cells (Novagen) and plated on LB-Chloramphenicol agar plates. Plasmids extracted from 
colonies were sequenced and ones with the correct sequences were transformed into MC1061 competent cells from Invitrogen. The final constructs were of the form (OmpX Signal sequence - Thrombin cleavage site - Cystine stabilized miniprotein - eCPX).

\section{Miniprotein primary sequences}

\section{1 - GGWGG in Min-23 scaffold: LMRCKQDSDCLAGSVCGGWGGFCG}

\section{2 - $\beta$-Trypsin inhibitor knottin: RVCPRILMECKKDSDCLAECVCLEHGYCG}

Bacterial Experiments. $5 \mathrm{~mL}$ media of $\mathrm{LB}+34 \mu \mathrm{g} / \mathrm{ml}$ chloramphenicol (Cam) were inoculated with glycerol stocks bacteria displaying 1 and $\mathbf{2}$ and incubated overnight at $37^{\circ} \mathrm{C}$ with $250 \mathrm{rpm}$ shaking. $100 \mu \mathrm{L}$ of these cultures were then added into fresh $5 \mathrm{~mL}$ LB-Cam media and allowed to grow for $2 \mathrm{~h}$ at $37^{\circ} \mathrm{C}, 250 \mathrm{rpm}$. This was then induced with $0.04 \%(w / v)$ L-arabinose and incubated at $37^{\circ} \mathrm{C}, 250 \mathrm{rpm}$ for $2 \mathrm{~h}$. For fluorescent microscopy related experiments, $1 \mu \mathrm{g} / \mathrm{ml}$ Hoechst 33342 dye was added and allowed to stain the cells for $10 \mathrm{~min}$. The cultures were then spun down at $6000 \mathrm{~g}$ for $10 \mathrm{~min}$. The supernatant was discarded, $5 \mathrm{~mL}$ HEPES (10 mM) NaCl (137 mM) buffer was added and the cells were resuspended. This was repeated 2 times to wash the cells. Finally HEPES $\mathrm{NaCl}$ buffer was added in appropriate quantities to provide a solution with desired bacterial density. Bacterial cell density was determined using a Biochrom WPA CO8000 Cell Density meter. An Olympus microscope IX71 with filters were used for recording fluorescent images. AFM images were recorded using an NTegra Spectra from NT-MDT. Real time images of bacterial aggregation were taken using a Veho VMS-004 deluxe microscopic camera. Image processing and analysis was done using ImageJ. The data was plotted using Microcal Origin 8.0.

Cucurbituril Solutions. Cucurbit[8]uril and cucurbit[7]uril were purchased from SigmaAldrich. Due to poor solubility of $\mathrm{CB}[8]$ in water and its hygroscopic nature, the apparent molecular weight of the commercial powder and its actual concentration in aqueous solutions were determined for each batch using a simple and highly reproducible method described previously. ${ }^{49}$

FG6 Peptide. The FG6 peptide was a gift from Emanuela Cavatorta, produced by solid phase peptide synthesis and purified using reverse phase HPLC.

Bacterial Viability Test. The bacterial viability test was conducted by preparing 3 separate solutions of bacteria displaying 1 having O.D.60o values of $1.0\left(\sim 10^{9}\right.$ cells $\left./ \mathrm{ml}\right)$. To one solution nothing was added, to the second $25 \mu \mathrm{M} \mathrm{CB} 8$ was added and to the third 
1mM FG6 peptide was added. These were incubated at room temperature for 4 hours and then $10^{5}, 10^{4}$ and $10^{3}$ cells $/ \mathrm{ml}$ dilutions were made for each of the samples. $10 \mu \mathrm{l}$ of these dilutions were placed as drops on top of LB Agar plates containing chloramphenicol as a selective antibiotic. The plate was then tilted to form 3 streams which quickly dried. This was then incubated overnight a $37^{\circ} \mathrm{C}$. As seen in Figure 8.7, all 3 plates showed comparable number of bacterial colonies in all dilutions indicating that neither $\mathrm{CB}[8]$ nor $\mathrm{FG} 6$ had any cytotoxic effects

a) Buffer

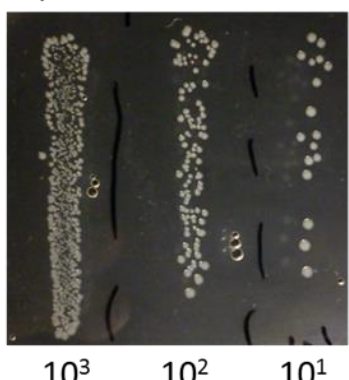

b) $25 \mu \mathrm{M} \mathrm{CB}[8]$

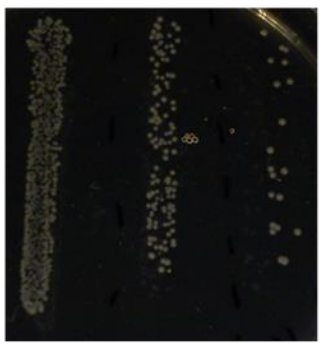

$10^{3} \quad 10^{2} \quad 10^{1}$ c) $1 \mathrm{mM}$ FG6

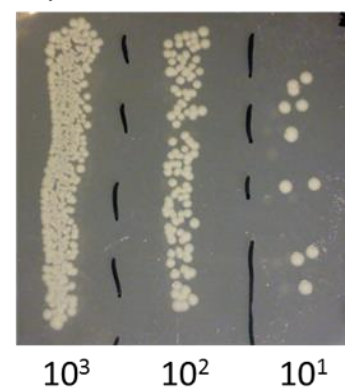

Figure 8.7. Bacterial viability test. Three individual agar plates with colonies formed from $10 \mu \mathrm{l}$ of diluted bacterial solutions mixed with a) only buffer, b) $25 \mu \mathrm{M} \mathrm{CB}$ [8] and c) $1 \mathrm{mM}$ FG6. The values below the lanes indicate number of bacterial cells per $10 \mu \mathrm{l}$

Preparation of SAMs on gold. Gold substrates were first washed with piranha solution $\left(\mathrm{H}_{2} \mathrm{SO}_{4}+30 \% \mathrm{H}_{2} \mathrm{O}_{2}, \mathrm{v} / \mathrm{v} 70 / 30\right)$, and copious amounts of Milli-Q water afterwards. Substrates were then immersed into a mixed solution of $0.1 \mathrm{mM}$ triethyleneglycol and 2-mercaptoethanol at a ratio of 99:1 (v/v) for 3 mins, washed with water, and dried with $\mathrm{N}_{2}$. The binary complex of methyl viologen thiol/CB[8] was formed at the concentration of $50 \mu \mathrm{M}$, and was back inserted into triethylene glycol modified gold surfaces by overnight incubation. After rinsing with water for $5 \mathrm{~min}$ the substrates were used for bacterial immobilization experiments.

Bacterial immobilization experiments. The $\mathrm{CB}[8]$ monolayers on gold were incubated with $\mathrm{H} 33342$ stained $0.01 \mathrm{OD}_{600 n m}$ bacterial solutions containing $50 \mu \mathrm{M} \mathrm{CB}[8]$ for 1 hour. The substrates were then washed thoroughly with water and dried. These cells were then visualized using and epifluorescence microscope.

Bacterial surface motility experiments. We used a macroscopic flow cell having $\mathrm{CB}[8]$ monolayers on a $20 \mathrm{~nm}$ thick 1 inch circular gold substrate on one side and a glass window on the other, enabling us to obtain images from either side. A solution 
containing $50 \mu \mathrm{M} \mathrm{CB}[8]$ and $0.01 \mathrm{OD}_{600 \mathrm{~nm}}$ bacteria displaying 1 was flowed into the chamber and the bacteria were allowed to bind without flow for $30 \mathrm{~min}$. This was followed by flushing the chamber with buffer and capturing images and videos of the motile cells in bright field mode. A $0.5 \mathrm{mM}$ FG6 solution was flowed into the chamber and incubated for 1 hour during which almost all the cells released. Video S7 (Section 8.7) was taken after 10 min of FG6 incubation when few cells were still seen attached.

\subsection{References}

(1) Ryu, J.-H., Lee, E., Lim, Y., and Lee, M. (2007) Carbohydrate-coated supramolecular structures: transformation of nanofibers into spherical micelles triggered by guest encapsulation. J. Am. Chem. Soc. 129, 4808-4814.

(2) Müller, M. K., and Brunsveld, L. (2009) A supramolecular polymer as a self-assembling polyvalent scaffold. Angew. Chem. Int. Ed Engl. 48, 2921-2924.

(3) Yu, G., Ma, Y., Han, C., Yao, Y., Tang, G., Mao, Z., Gao, C., and Huang, F. (2013) A sugarfunctionalized amphiphilic pillar[5]arene: synthesis, self-assembly in water, and application in bacterial cell agglutination. J. Am. Chem. Soc. 135, 10310-10313.

(4) Lui, L. T., Xue, X., Sui, C., Brown, A., Pritchard, D. I., Halliday, N., Winzer, K., Howdle, S. M., Fernandez-Trillo, F., Krasnogor, N., and Alexander, C. (2013) Bacteria clustering by polymers induces the expression of quorum-sensing-controlled phenotypes. Nat Chem 5, 1058-1065.

(5) Rozhok, S., Shen, C. K.-F., Littler, P.-L. H., Fan, Z., Liu, C., Mirkin, C. A., and Holz, R. C. (2005) Methods for fabricating microarrays of motile bacteria. Small 1, 445-451.

(6) Hiratsuka, Y., Miyata, M., Tada, T., and Uyeda, T. Q. P. (2006) A microrotary motor powered by bacteria. Proc. Natl. Acad. Sci. 103, 13618-13623.

(7) Gartner, Z. J., and Bertozzi, C. R. (2009) Programmed assembly of 3-dimensional microtissues with defined cellular connectivity. Proc. Natl. Acad. Sci. 106, 4606-4610.

(8) Zope, H. R., Versluis, F., Ordas, A., Voskuhl, J., Spaink, H. P., and Kros, A. (2013) In vitro and in vivo supramolecular modification of biomembranes using a lipidated coiled-coil motif. Angew. Chem. Int. Ed. 52, 14247-14251.

(9) Dutta, D., Pulsipher, A., Luo, W., and Yousaf, M. N. (2011) Synthetic chemoselective rewiring of cell surfaces: Generation of three-dimensional tissue structures. J. Am. Chem. Soc. 133, 8704-8713.

(10) Aida, T., Meijer, E. W., and Stupp, S. I. (2012) Functional supramolecular polymers. Science 335, 813-817.

(11) Fenske, T., Korth, H.-G., Mohr, A., and Schmuck, C. (2012) Advances in switchable supramolecular nanoassemblies. Chem. - Eur. J. 18, 738-755.

(12) Yang, L., Gomez-Casado, A., Young, J. F., Nguyen, H. D., Cabanas-Danés, J., Huskens, J., Brunsveld, L., and Jonkheijm, P. (2012) Reversible and oriented immobilization of ferrocenemodified proteins. J. Am. Chem. Soc. 134, 19199-19206.

(13) Uhlenheuer, D. A., Petkau, K., and Brunsveld, L. (2010) Combining supramolecular chemistry with biology. Chem. Soc. Rev. 39, 2817-2826.

(14) Brinkmann, J., Cavatorta, E., Sankaran, S., Schmidt, B., van Weerd, J., and Jonkheijm, P. (2014) About supramolecular systems for dynamically probing cells. Chem. Soc. Rev. 43, 44494469.

(15) Lagona, J., Mukhopadhyay, P., Chakrabarti, S., and Isaacs, L. (2005) The cucurbit[n]uril family. Angew. Chem. Int. Ed. 44, 4844-4870. 
(16) Masson, E., Ling, X., Joseph, R., Kyeremeh-Mensah, L., and Lu, X. (2012) Cucurbituril chemistry: a tale of supramolecular success. RSC Adv. 2, 1213-1247.

(17) Hou, C., Li, J., Zhao, L., Zhang, W., Luo, Q., Dong, Z., Xu, J., and Liu, J. (2013) Construction of protein nanowires through cucurbit[8]uril-based highly specific host-guest interactions: an approach to the assembly of functional proteins. Angew. Chem. Int. Ed. 52, 5590-5593.

(18) Hennig, A., Bakirci, H., and Nau, W. M. (2007) Label-free continuous enzyme assays with macrocycle-fluorescent dye complexes. Nat. Methods 4, 629-632.

(19) Ko, Y. H., Kim, E., Hwang, I., and Kim, K. (2007) Supramolecular assemblies built with hoststabilized charge-transfer interactions. Chem. Commun. 1305-1315.

(20) Appel, E. A., del Barrio, J., Loh, X. J., and Scherman, O. A. (2012) Supramolecular polymeric hydrogels. Chem. Soc. Rev. 41, 6195-6214.

(21) González-Campo, A., Brasch, M., Uhlenheuer, D. A., Gómez-Casado, A., Yang, L., Brunsveld, L., Huskens, J., and Jonkheijm, P. (2012) Supramolecularly oriented immobilization of proteins using cucurbit[8]uril. Langmuir 28, 16364-16371.

(22) Appel, E. A., Rowland, M. J., Loh, X. J., Heywood, R. M., Watts, C., and Scherman, O. A. (2012) Enhanced stability and activity of temozolomide in primary glioblastoma multiforme cells with cucurbit[n]uril. Chem. Commun. 48, 9843-9845.

(23) Kim, E., Kim, D., Jung, H., Lee, J., Paul, S., Selvapalam, N., Yang, Y., Lim, N., Park, C. G., and Kim, K. (2010) Facile, template-free synthesis of stimuli-responsive polymer nanocapsules for targeted drug delivery. Angew. Chem. Int. Ed. 49, 4405-4408.

(24) Ma, D., Hettiarachchi, G., Nguyen, D., Zhang, B., Wittenberg, J. B., Zavalij, P. Y., Briken, V., and Isaacs, L. (2012) Acyclic cucurbit[n]uril molecular containers enhance the solubility and bioactivity of poorly soluble pharmaceuticals. Nat. Chem. 4, 503-510.

(25) Heo, S. W., Choi, T. S., Park, K. M., Ko, Y. H., Kim, S. B., Kim, K., and Kim, H. I. (2011) Hostguest chemistry in the gas phase: selected fragmentations of $\mathrm{CB}[6]$-peptide complexes at lysine residues and its utility to probe the structures of small proteins. Anal. Chem. 83, 7916-7923.

(26) Ghale, G., Ramalingam, V., Urbach, A. R., and Nau, W. M. (2011) Determining protease substrate selectivity and inhibition by label-free supramolecular tandem enzyme assays. J. Am. Chem. Soc. 133, 7528-7535.

(27) Chinai, J. M., Taylor, A. B., Ryno, L. M., Hargreaves, N. D., Morris, C. A., Hart, P. J., and Urbach, A. R. (2011) Molecular recognition of insulin by a synthetic receptor. J. Am. Chem. Soc. 133, $8810-8813$.

(28) Logsdon, L. A., and Urbach, A. R. (2013) Sequence-specific inhibition of a nonspecific protease. J. Am. Chem. Soc. 135, 11414-11416.

(29) Heitmann, L. M., Taylor, A. B., Hart, P. J., and Urbach, A. R. (2006) Sequence-specific recognition and cooperative dimerization of $\mathrm{N}$-terminal aromatic peptides in aqueous solution by a synthetic host. J. Am. Chem. Soc. 128, 12574-12581.

(30) Dang, D. T., Nguyen, H. D., Merkx, M., and Brunsveld, L. (2013) Supramolecular control of enzyme activity through cucurbit[8]uril-mediated dimerization. Angew. Chem. Int. Ed. 52, 29152919.

(31) Dang, D. T., Schill, J., and Brunsveld, L. (2012) Cucurbit[8]uril-mediated protein homotetramerization. Chem. Sci. 3, 2679-2684.

(32) Nguyen, H. D., Dang, D. T., van Dongen, J. L. J., and Brunsveld, L. (2010) Protein dimerization induced by supramolecular interactions with cucurbit[8]uril. Angew. Chem. Int. Ed. 49, 895-898.

(33) Rowland, M. J., Appel, E. A., Coulston, R. J., and Scherman, O. A. (2013) Dynamically crosslinked materials via recognition of amino acids by cucurbit[8]uril. J. Mater. Chem. B 1, 29042910. 
(34) Uhlenheuer, D. A., Young, J. F., Nguyen, H. D., Scheepstra, M., and Brunsveld, L. (2011) Cucurbit[8]uril induced heterodimerization of methylviologen and naphthalene functionalized proteins. Chem. Commun. 47, 6798-6800.

(35) Tian, F., Jiao, D., Biedermann, F., and Scherman, O. A. (2012) Orthogonal switching of a single supramolecular complex. Nat Commun 3, 1207.

(36) Rice, J. J., and Daugherty, P. S. (2008) Directed evolution of a biterminal bacterial display scaffold enhances the display of diverse peptides. Protein Eng. Des. Sel. 21, 435-442.

(37) Getz, J. A., Rice, J. J., and Daugherty, P. S. (2011) Protease-resistant peptide ligands from a knottin scaffold library. ACS Chem. Biol. 6, 837-844.

(38) Bush, M. E., Bouley, N. D., and Urbach, A. R. (2005) Charge-mediated recognition of nterminal tryptophan in aqueous solution by a synthetic host. J. Am. Chem. Soc. 127, 14511-14517.

(39) Sonzini, S., Ryan, S. T. J., and Scherman, O. A. (2013) Supramolecular dimerisation of middlechain Phe pentapeptides via $\mathrm{CB}[8]$ host-guest homoternary complex formation. Chem. Commun. 49, 8779-8781.

(40) Harada, A., Kobayashi, R., Takashima, Y., Hashidzume, A., and Yamaguchi, H. (2011) Macroscopic self-assembly through molecular recognition. Nat Chem 3, 34-37.

(41) Ahn, Y., Jang, Y., Selvapalam, N., Yun, G., and Kim, K. (2013) Supramolecular velcro for reversible underwater adhesion. Angew. Chem. Int. Ed. 52, 3140-3144.

(42) Bode, W., Greyling, H. J., Huber, R., Otlewski, J., and Wilusz, T. (1989) The refined 2.0 A X-ray crystal structure of the complex formed between bovine beta-trypsin and CMTI-I, a trypsin inhibitor from squash seeds (Cucurbita maxima). Topological similarity of the squash seed inhibitors with the carboxypeptidase A inhibitor from potatoes. FEBS Lett. 242, 285-292.

(43) Bush, M. E., Bouley, N. D., and Urbach, A. R. (2005) Charge-mediated recognition of Nterminal tryptophan in aqueous solution by a synthetic host. J. Am. Chem. Soc. 127, 14511-14517.

(44) Heitmann, L. M., Taylor, A. B., Hart, P. J., and Urbach, A. R. (2006) Sequence-specific recognition and cooperative dimerization of $\mathrm{N}$-terminal aromatic peptides in aqueous solution by a synthetic host. J. Am. Chem. Soc. 128, 12574-12581.

(45) Sonzini, S., Ryan, S. T. J., and Scherman, O. A. (2013) Supramolecular dimerisation of middlechain Phe pentapeptides via $\mathrm{CB}[8]$ host-guest homoternary complex formation. Chem. Commun. 49, 8779-8781.

(46) Thomas, G. B., Rader, L. H., Park, J., Abezgauz, L., Danino, D., DeShong, P., and English, D. S. (2009) Carbohydrate modified catanionic vesicles: probing multivalent binding at the bilayer interface. J. Am. Chem. Soc. 131, 5471-5477.

(47) An, Q., Brinkmann, J., Huskens, J., Krabbenborg, S., de Boer, J., and Jonkheijm, P. (2012) A supramolecular system for the electrochemically controlled release of cells. Angew. Chem. Int. Ed. 51, 12233-12237.

(48) Kudernac, T., Ruangsupapichat, N., Parschau, M., Macia, B., Katsonis, N., Harutyunyan, S. R., Ernst, K.-H., and Feringa, B. L. (2011) Electrically driven directional motion of a four-wheeled molecule on a metal surface. Nature 479, 208-211.

(49) Yi, S., and Kaifer, A. E. (2011) Determination of the purity of cucurbit[n]uril $(n=7,8)$ host samples. J. Org. Chem. 76, 10275-10278. 


\subsection{Videos}

The videos from which the plots of Figures 8.4, 8.5 and 8.6 were derived have been provided as multimedia files and can be downloaded at http://pubs.acs.org/doi/suppl/10.1021/acsnano.5bo0694. The timespans of all the videos correspond to the $\mathrm{x}$-axis values in the corresponding Figures.

Video 8.1 - Bacterial aggregation seen in bacterial strain displaying 1 but not observed in the strain displaying 2.

Video 8.2 - Aggregation requires ternary complex formation. $C B[8]$ induces aggregation while $\mathrm{CB}[7]$ does not. Upper captions indicate which cuvette had $25 \mu \mathrm{M}$ $\mathrm{CB}[8]$ and which one had $25 \mu \mathrm{M} \mathrm{CB}[7]$.

Video 8.3 - Rate of aggregation is dependent of $C B$ [8] concentration. Values on the top denote $\mathrm{CB}[8]$ concentrations mixed with 1.0 O.D. ${ }_{600}$ bacterial solutions.

Video 8.4 - Rate of aggregation is also dependent of bacterial density. Values on the top denote bacterial densities in O.D. ${ }_{600}$ values mixed with $25 \mu \mathrm{M} \mathrm{CB}[8]$.

Video 8.5 - Aggregation is reversible. First, aggregation of the bacterial strain displaying 1 (1.0 O.D. ${ }_{600}$ ) in the presence of $25 \mu \mathrm{M} \mathrm{CB}[8]$ is shown. Second, different concentrations of FG6 peptide mixed with the same bacterial solutions are shown. Reaggregation is inhibited depending on FG6 concentration. Values on the top denote FG6 concentrations in $\mu \mathrm{M}$.

Video 8.6 - Motility of bacteria immobilized on $\mathrm{CB}[8]$ monolayers. The video shows motility after 4 hours of immobilization.

Video 8.7 - Motility of bacteria immobilized on $\mathrm{CB}[8]$ monolayers after 10 mins of incubation with $0.5 \mathrm{mM} \mathrm{FG6.}$ 


\section{Chapter 9}

\section{Epilogue}

Section 9.2 was done in collaboration with Leena Jaatinen, Prof. Janos Vörös and Dr. Tomaso Zambelli from the Laboratory of Biosensors and Bioelectronics, ETH Zürich and Jenny Brinkmann from the University of Twente.

Section 9.3 was done with Nora Weineisen, Rianne Hommersom and Dr. Jens Voskuhl and is part of the manuscript: N.L. Weineisen, S. Sankaran, ${ }^{\#}$ C.A. Hommersom, ${ }^{\#}$ J. Voskuhl," N. Katsonis, P. Jonkheijm and J.J.L.M. Cornelissen; "Photo-responsive, reversible immobilization of virus particles on supramolecular platform". In preparation.

Section 9.4 was done in collaboration with Bettina Schmidt and Dr. Jens Voskuhl and is part of the manuscript: J. Voskuhl," B. Schmidt," S. Sankaran, L. Stegemann, C. Strassert, J. Ak, M. Waller, P. Jonkheijm; "Mannose-functionalized fluorescent aggregates of Thiophthalonitriles interact with bacteria"; Submitted to Chem. Commun., 2015.

\# Equal Authorship 


\subsection{Introduction}

The exploration of combining bacterial and supramolecular components to develop novel systems such as those that are described in this thesis provide a glimpse of the versatility offered by the combination. Each component and concept of this toolbox can be used interchangeably and also applied to various other fields. For instance, components such as cucurbit[8]uril ( $\mathrm{CB}[8]), \beta$-cyclodextrin, self-assembled monolayers (SAMs), supported lipid bilayers and cysteine-stabilized miniproteins and concepts like reversible binding, multivalency, aggregation and stimuli-responsiveness have been used in different combinations in each chapter to demonstrate the construction of different supramolecular bacterial systems. Obviously, there exists a vast ocean of possibilities to expand upon these systems and acquire new insights into various molecular and macromolecular processes involved. Such efforts are being made by our group and others resulting in the rapid development of associated multi-disciplinary fields. With this perspective, this epilogue summarizes preliminary results from a few studies being conducted to explore future avenues in supramolecular biological systems.

\subsection{Cell-adhesion force spectroscopy on supramolecular surfaces}

Self-assembled monolayers of $\mathrm{CB}[8]$ and its associated guests have been employed in Chapters 3 and 8 of this thesis. These monolayers allow the presentation of bioactive ligands in a dynamic and reversible manner, highly suitable for biological studies. Apart from bacteria, such SAMs have also been employed to address mammalian cells. Our group has employed $\mathrm{CB}[8]-\mathrm{MV}^{2+} \mathrm{SAMs}$ along with a second guest that displays the integrin-binding peptide Arg-Gly-Asp (RGD) for the construction of an electrochemically responsive platform for cell adhesion. ${ }^{1,2}$ Electrochemical reduction of $\mathrm{MV}^{2+}$ to the radical cation of methyl viologen $\left(\mathrm{MV}^{+}\right)$results in the disassembly of the ternary complex, causing adhered cells to be released from the surface. Using microelectrodes, release of only parts of a cell from the surface has also been achieved. ${ }^{1,2}$ Such a platform has great potential in studying cell adhesion and migration processes and can also be used to provide additional functionality to improve medical implants like stents.

However, the non-covalent interactions between individual components involved in such supramolecular surfaces are relatively weak $(1-10 \mu \mathrm{M})^{3,4}$ with a high rate of association $\left(k_{a}=9.6 \times 10^{7} \mathrm{M}^{-1} \mathrm{~s}^{-1}\right)$ and rapid rate of dissociation $\left(k_{d}=1200 \mathrm{~s}^{-1}\right)$ for the second guest, naphthol (Np). ${ }^{5}$ This could potentially suggest that cell adhesion does not occur on these non-covalently assembled surfaces to the same extent as it does on 202 
more traditional surfaces where ligands are immobilized in a covalent manner. ${ }^{6-9}$ Through fluorescent labelling, it was determined that mouse myoblast cells $\left(\mathrm{C}_{2} \mathrm{C}_{12}\right)$ adhering to surfaces with both covalently and non-covalently linked ligands had wellformed focal adhesions and actin networks during the course of several hours (Figure 9.1). This indicated that the cell contractility on our supramolecular surfaces could be comparable to surfaces with covalently immobilized ligands.
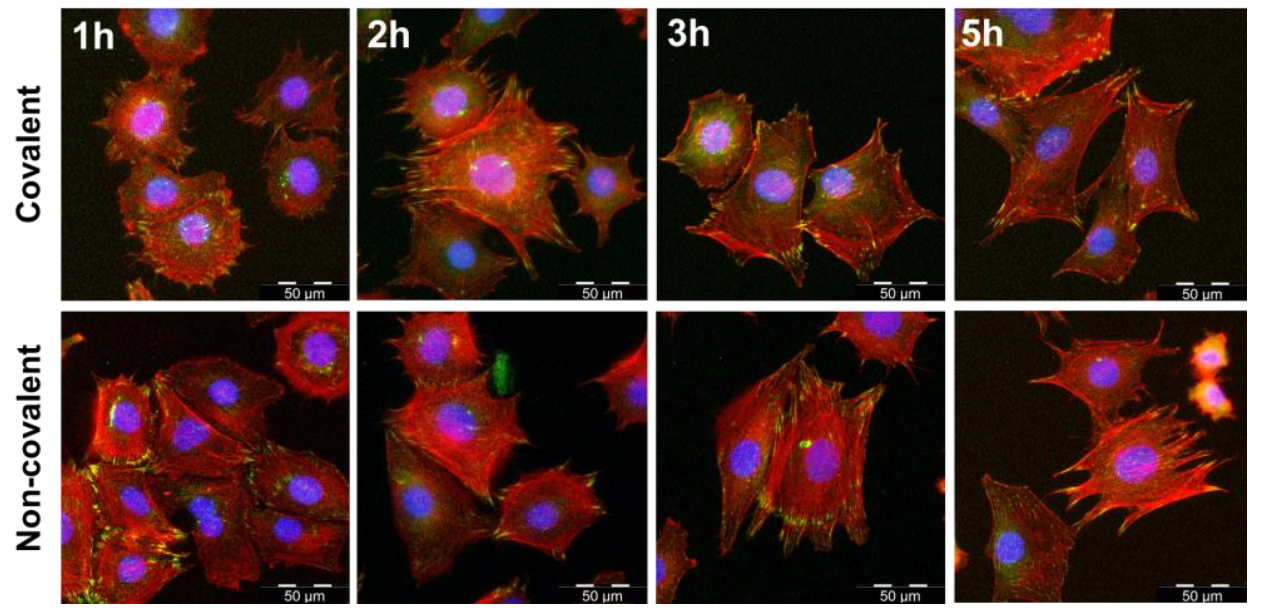

Figure 9.1. Fluorescent overlaid images of $\mathrm{C}_{2} \mathrm{C} 12$ cells on the covalent and non-covalent surfaces, stained to visualize actin (red), focal adhesion protein vinculin (green) and cell nuclei (blue). Timepoints indicated on the top left of the images correspond to number of hours after cell seeding.

To gain a better understanding of the forces involved between adhered cells and supramolecular surfaces, we used a relatively new technique called FluidFM. ${ }^{10}$ As shown in Figure 9.2a, it utilizes an atomic force microscopy (AFM) cantilever with an integrated microchannel. The device functions as an AFM with the possibility to perform aspiration, by applying negative pressure and dispensing, by applying positive pressure through the microchannel like a miniscule pipette. For cell-adhesion force measurements, ${ }^{11}$ the cantilever is slowly lowered until the opening of the microchannel makes contact with a cell, which is detected by a slight upward bending of the cantilever. A negative fluid pressure is then applied in the microchannel to "grip" the cell, after which the cantilever is slowly raised again, resulting in the cell being pulled off the surface as shown in Figure 9.2b. As the cantilever is raised, it gets bent downwards since the cell is still "stuck" to the surface with a certain force. Once the force required to bend the cantilever exceeds the maximum cell-adhesion force, a 
rupture event occurs, causing the cell to be picked up from the surface and the cantilever to return to its original shape. The bending of the cantilever is measured by optical beam deflection (OBD) in which an AFM laser beam is aligned to reflect off of the cantilever's tip onto a photo-detector. ${ }^{12}$ The position of the beam on the detector is measured in volts $(V)$. To determine the distance by which the cantilever bends, the sensitivity (S) is measured beforehand in $\mathrm{nm} / \mathrm{V}$ by lowering the cantilever on a hard surface and allowing it to bend. This distance can then be converted into force $(F)$ using the spring constant of the cantilever $(k)$ determined using the Sader method ${ }^{13}$ with the units $\mathrm{nN} / \mathrm{nm}$. Consequently, the force is measured using eq 9.1.

$$
F=V S k \quad \text { eq } 9.1
$$

A typical force spectroscopy curve from such cell pick-up experiments is presented in Figure 9.2c, in which the initial drop in force represents the bending of the cantilever that is being raised after it has "gripped" a cell. The rupture force is the point where the force to bend the cantilever equals the cell-adhesion force. Beyond this point, the cantilever picks up the cell and returns to its original shape, represented by the force curve returning to its initial value. The total distance required to pull the cell off the surface is measured from the initial point where the cantilever bending began to when it reaches its original shape once more. The area of the shaded region in the forcedistance curve represents the work done by the cantilever to remove the cell from the surface and corresponds to the binding energy of the cell with the surface.

Using this tool, we performed cell-adhesion force measurements on two types of RGDpresenting surfaces. Both surfaces had a well-packed non-fouling tetraethylene glycol background monolayer with a reactive maleimide group displayed over $1 \%$ of the surface (Figure 9.2a). On the "non-covalent" surfaces, a thiolated $\mathrm{MV}^{2+}$ was conjugated to the maleimides to which $\mathrm{CB}[8]$ was able to bind. An RGD peptide conjugated with a naphthol unit (Np-RGD) acted as the second guest and allowed RGD to be presented on the surface in a non-covalent manner. On the "covalent" surfaces, an RGD peptide with a cysteine residue was conjugated directly to the maleimide. Mouse myoblast $\mathrm{C}_{2} \mathrm{C}_{12}$ cells were allowed to adhere to these surfaces and spread for an hour after which pickup experiments were done for a time period of up to 4 hours. In total 49 cells were successfully picked up from non-covalent surfaces and 40 cells from covalent surfaces. From Figure 9.2d-f, it can be seen that the force, distance and work required to pick-up the cells from both surfaces are very similar. Since the data sets follow Gaussian distribution with outliers, the non-parametric Mann-Whitney test was applied and it was determined that significant differences did not exist between the two types of 204 
surfaces for all 3 parameters. These results suggest that, even though the components in the non-covalent surfaces are held together by relatively weak forces, cells are still able to adhere considerably well. In the covalent system, the weakest link in celladhesion is the non-covalent interaction between RGD and integrins on the cell surface. The rupture force measured during cell pick-up experiments would correspond to the breaking of all these interactions. Since the rupture force in the non-covalent system is similar to that of the covalent system, it suggests that the supramolecular ternary complex is comparably or more stable when compared to the RGD-integrin complex. Force measurement studies of adamantanes (Ad) with $\beta$-cyclodextrin $(\beta-C D)$ have shown that the rupture force of the Ad- $\beta-C D$ complex is about $100 \mathrm{pN} .{ }^{14}$ Since the association and dissociation rate constants of this complex $\left(k_{a}=10^{8} \mathrm{M}^{-1} \mathrm{~s}^{-1}, k_{d}=2000 \mathrm{~s}^{-}\right.$ $\left.{ }^{1}\right)^{14}$ is in a similar range to the rate constants between $\mathrm{Np}$ and the $\mathrm{CB}[8]-\mathrm{MV}^{2+}$ complex $\left(k_{a}=9.6 \times 10^{7} \mathrm{M}^{-1} \mathrm{~s}^{-1}, k_{d}=1200 \mathrm{~s}^{-1}\right),{ }^{5}$ it is fair to assume that the rupture force of the ternary complex could be comparable based on a theory developed by Evans et al. ${ }^{15}$ On the other hand, force spectroscopy studies of individual RGD-integrin complexes have shown that the rupture forces required to disassemble this complex are generally less than $100 \mathrm{pN}^{16-19}$ This supports the idea that individual RGD-integrin interactions are weaker than the non-covalent interactions involved in individual supramolecular ternary complexes, indicating that the measured rupture forces correspond to the rupture of the RGD-integrin complexes in both the covalent and non-covalent systems. These results go to show that such non-covalent systems, with dynamic and responsive components, can be constructed with similar cell-adhesive properties compared to relatively passive but conventional covalent systems. So far, these experiments have indicated that actin filaments, focal adhesions, adhesion forces and cell contractility between cells adhering to our covalent and non-covalent surfaces are comparable. ${ }^{1,2}$ However preliminary experiments in our group dealing with cell motility indicate that the extent of dynamism in the ligand display might lead to different migratory profiles. Further investigations are being conducted by modulating the bioactive ligand's binding strength, valency and stimuli-responsiveness to understand the effects of these parameters on cell contractility and migration. 

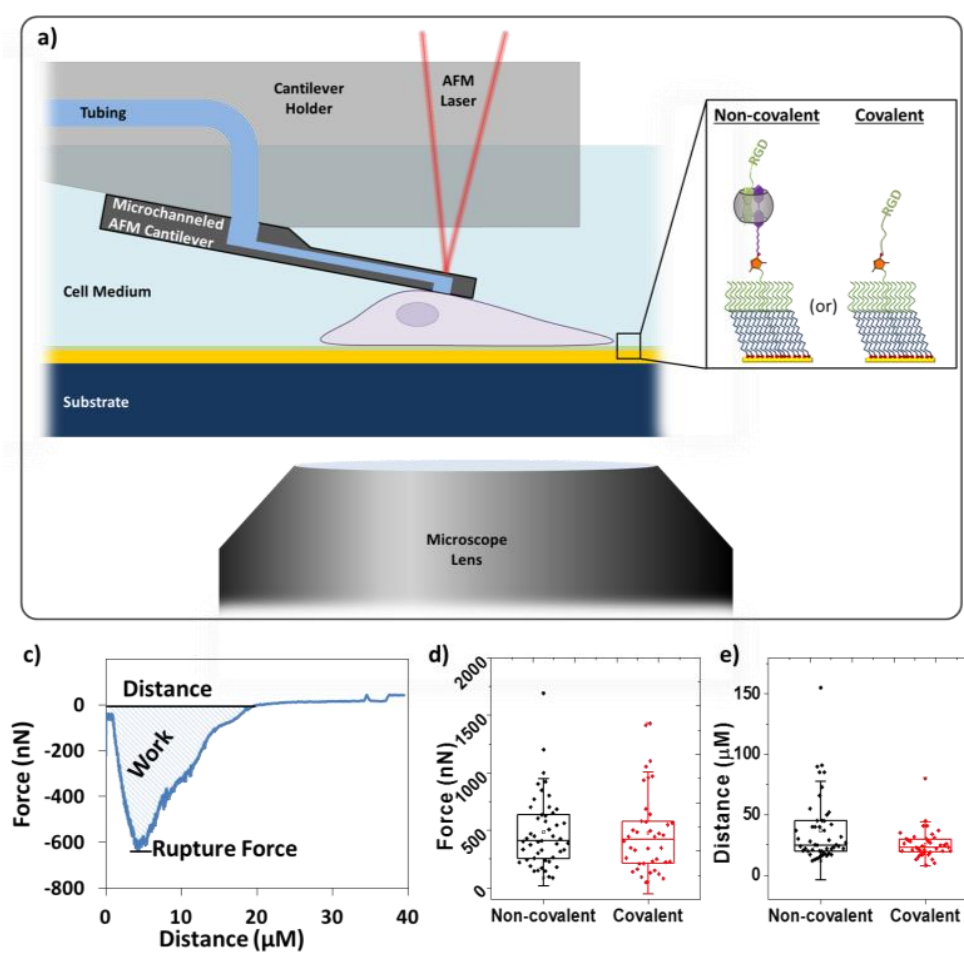
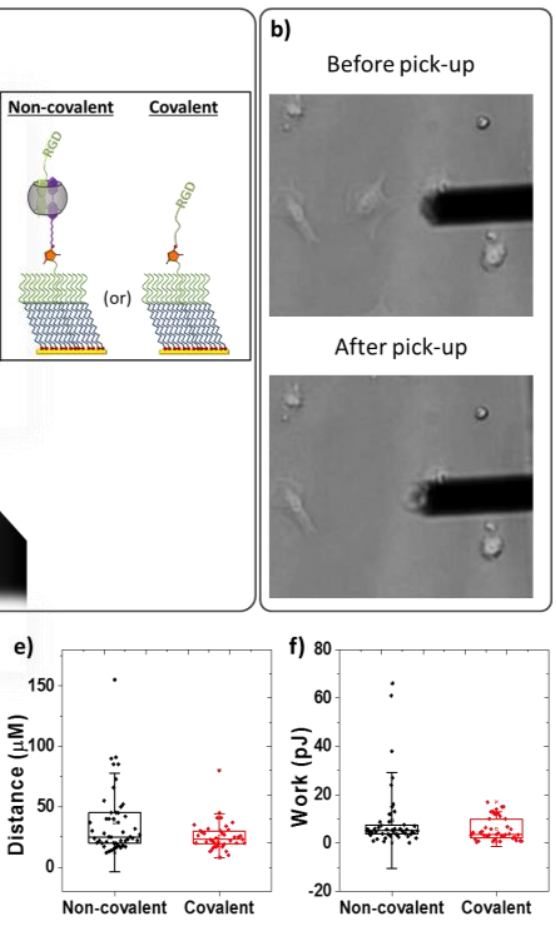

Figure 9.2. a) Schematic depicting the FluidFM system for cell-adhesion force spectroscopy. b) Representative pictures from a cell pick-up experiment. The black bar is the micro-channeled cantilever and the cell immediately to the left of it in the "Before pick-up" image is the one that was picked up. c) Representative Force-Distance plot beginning right after the cantilever gripped the cell. Box plots of the d) rupture force, e) distance and f) work done in the cell pick-up experiments (49 cell in Non-covalent, 40 cells in Covalent). The top and bottom of the boxes correspond to the first and third quartiles, the line in the middle corresponds to the median and the whiskers represent the standard deviation of the data sets.

\subsection{Supramolecular viral protein cages}

Self-assembled viral protein cages like cowpea chlorotic mottle virus (CCMV) are widely used in material sciences, ${ }^{20}$ medicine ${ }^{21}$ and catalysis. ${ }^{22}$ These particles are symmetrical and monodisperse, can encapsulate functional cargo ${ }^{23,24}$ and have been decorated with a variety of drugs, fluorescent dyes, polymers and carbohydrates. ${ }^{25-28}$ CCMV is an icosahedral plant virus consisting of 180 identical coat proteins that self-assemble around the viral RNA. The spherical capsid is $28 \mathrm{~nm}$ in diameter. A particularly interesting feature of CCMV, is its defined and reversible assembly behaviour. ${ }^{29}$ 
Depending on $\mathrm{pH}$ and ionic strength, CCMV can disassemble into coat protein dimers and reassemble into non-infectious virus-like-particles (VLP). This makes it possible to use CCMV as an encapsulation vesicle. Reversible surface immobilization of these viral protein cages would enable them to be incorporated with medical implants after which they can be released when required for targeted deliver of their encapsulated cargo. Hommersom et al. recently developed a strategy for the functionalization of CCMV via the copper-catalyzed azide-alkyne cycloaddition (CuAAC). ${ }^{30}$ Here, we use this strategy to functionalize CCMV coat proteins with azobenzene (azo), which then enabled them to be immobilized in a photoresponsive manner to $C B[8]$ monolayers described in section 9.2 (Figure 9.3a).

After functionalization and characterization of the CCMV-azo viral protein cage, its interaction with $\mathrm{CB}[8]$ monolayers was investigated using surface plasmon resonance (SPR) measurements. The $\mathrm{MV}^{2+}$-surface was incubated stepwise with $\mathrm{CB}[8]$ and increasing concentrations of the virus capsid (0.5-5.0 $\mu \mathrm{M}$ apparent azo concentrations) to determine the association constant. Incubation with $\mathrm{CB}[8]$ led to a slight increase of the SPR signal indicating the 1:1 complex formation with MV. Next, the surface was incubated with increasing concentrations of CCMV-azo, resulting in a stepwise increase of the SPR signal due to the heteroternary complex formation between $\mathrm{MV}^{2+}, \mathrm{CB}[8]$ and azo (Figure 9.3b). Plotting the response against corresponding apparent azo concentrations provided a plot that was fitted with the Hill equation (Figure 9.3c). The obtained dissociation constant $\left(K_{d}=1.36 \mu \mathrm{M}, \mathrm{n}=2.2\right)$ is over an order of magnitude higher than a monovalent interaction of azo with the $\mathrm{CB}[8]-\mathrm{MV}^{2+}$ complex $\left(\mathrm{K}_{d}=70\right.$ $\mu M) .{ }^{31}$ We were also unable to fit the plot with the Langmuir adsorption equation or the Hill equation having $n=1$ since the linear regime of the plot rose too sharply and reached saturation over a very short range of concentrations (within 1 order of magnitude). These characteristics led us to believe that more than one azo is accessible in the contact area between the virus and the $C B[8]$ monolayer, resulting in a multivalent interaction. Furthermore the dynamics and reversibility of the binding of CCMV-CoumAzo to the $\mathrm{CB}[8]$ surface were checked. The surface was first washed with buffer and $C B[8]$, showing only negligible removal of virus. However, when the surface was incubated with (ferrocenylmethyl)trimethyl-ammonium (FcTMA+), which is a competitive inhibitor for $\mathrm{CB}[8]$, a significant decrease in the SPR signal was observed. Control experiments using $\mathrm{CB}[7]$ instead of $\mathrm{CB}[8]$ show no significant response because $\mathrm{CB}[7]$ is not able to include both guests. In a second control experiment native CCMV was incubated on a $C B[8]$ surface. Also in this case no significant change in SPR signal was observed, demonstrating the evident role of azobenzene in our system. 
The reversible immobilization, as described above, was subsequently visualized using microcontact printed $C B[8]$ arrays (Figure 9.3d, e). To visualize the viral protein cages, the viral RNA was stained with SYBR ${ }^{\circledR}$ Safe DNA Gel Stain yielding a green fluorescence. In the first control experiments native $C \mathrm{CMV}$ was incubated with arrays of either $\mathrm{CB}[7]$ or $\mathrm{CB}[8]$ for $10 \mathrm{~min}$ (Ctrl1 and Ctrl2, Figure 9.3d). The surface was then washed, stained and analyzed by fluorescence microscopy. In both experiments negligible fluorescence was detected, hence the particles do not bind non-specifically to the $C B[n]$ surfaces. Next, CCMV-azo was incubated with $\mathrm{CB}[7]$ arrays, which, as expected, also showed no significant fluorescence (Ctrl3, Figure 9.3d). In the final experiment cis- and trans-CCMVazo were incubated with $\mathrm{CB}[8]$ arrays. In the case of cis-CCMV-azo the sample was irradiated with UV light $(\lambda=365 \mathrm{~nm})$ prior to use, which is known to achieve $\sim 70 \%$ photoisomerization from trans to cis. Since cis-azo is more hydrophilic and bulkier it is not able to form the charge transfer complex with $\mathrm{MV}^{2+}$ within $\mathrm{CB} 8$, hence no binding occurs. $^{32}$ Therefore only faint fluorescent patterns were observed possibly due to residual trans-CCMV-azo (Figure 9.3d). Upon incubation of the $\mathrm{CB}[8]$ arrays with transCCMV-azo clear circle patterns were visible (+, Figure 9.3e). These results prove that the immobilization of CCMV-azo on the $\mathrm{CB}[8]$ monolayers occurs in a specific manner. Furthermore, photoisomerization experiments were performed to check the reversibility of the binding of $\mathrm{CCMV}$-azo on the $\mathrm{CB}[8]$ monolayers. Therefore the specifically adhered arrays of virus particles were irradiated with UV light (Figure 9.3e). We first confirmed that the irradiation would not significantly bleach the fluorescent arrays by irradiating them in air (UV-air), finding that the intensity dropped by about $25 \%$. Next, we confirmed that incubation of the substrates in virus buffer (VB, Figure 9.3e) does not cause significant dissociation of the virus particles over $5 \mathrm{~min}(<10 \% \mathrm{drop}$ in fluorescence). Irradiation of the arrays for $5 \mathrm{~min}$ in virus buffer (UV-VB) caused a substantial drop in fluorescence ( $90 \%)$ indicating that trans-cis isomerization of the azo moieties resulted in release of the virus particles from the surface. Localized release of CCMV-CoumAzo was also attempted by irradiating only a small region within the field of view. The fluorescence intensity of arrays within this region dropped by $\sim 60 \%$ (In, Figure 9.3e), while the arrays around this irradiated spot had a drop of only $\sim 20 \%$ (Out, Figure 9.3e), probably due to scattering of the irradiation. Clearly, selective photo responsive release of CCMV-azo was achieved. 

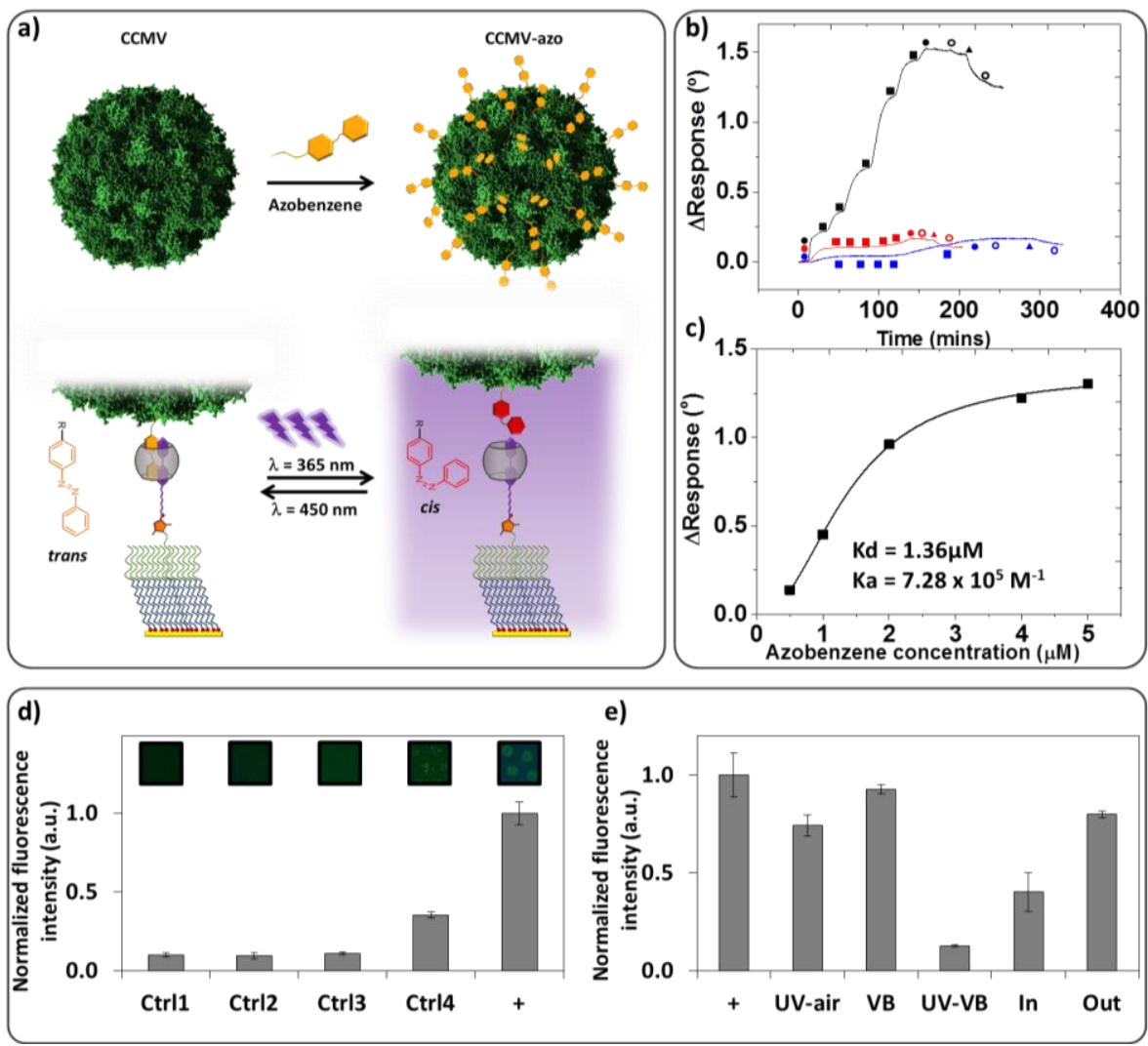

Figure 9.3. a) Schematic representing the functionalization of CCMV with azo resulting in the construct CCMV-azo. Supramolecular surface adhesion of trans-CCMV-azo on CB[8] monolayers has been depicted, followed by its dissociation from the surface due to photoisomerization into cisCCMV-azo in the presence of $\lambda=365 \mathrm{~nm}$ light. b) SPR sensogram of the binding of CCMV-azo (black) and $C C M V$ (red) on $\mathrm{CB}[8]$ monolayers and $C \mathrm{CMV}$-azo on $\mathrm{CB}[7]$ monolayers (blue). The shapes represent the injection time-points of different solutions in the SPR flow cell: buffer ( $\mathbf{0}), C B[8] / C B[7]$

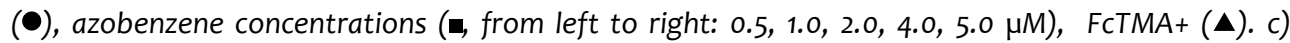
Binding curve of $C \mathrm{CMV}$-CoumAzo on a $\mathrm{CB}[8]$ monolayer fitted with the Hill equation. d) Microcontact printing of $C C M V$ on a $\mathrm{CB}[7]$ monolayer (Ctrl1), $\mathrm{CCMV}$ on a $\mathrm{CB}[8]$ monolayer (Ctrl2), trans-CCMV-azo on a $\mathrm{CB}[7]$ monolayer (Ctrl3), cis-CCMV-azo on a $\mathrm{CB}[8]$ monolayer (Ctrl4), trans-CCMV-azo on a $\mathrm{CB}[8]$ monolayer ( + ). Data presented as mean $\pm S E, n=4$. Images above columns represent $a$ width of 400 $\mu \mathrm{m}$. e) Release of $C \mathrm{CMV}$-azo from $\mathrm{CB}[8]$ patterns by UV irradiation under the following conditions: Initial state (no irradiation) (+), irradiation in air (UV-air), substrate in VB without irradiation (VB), irradiation in VB (UV-VB), irradiation in a localized spot (In), region outside the irradiated spot (Out). Data presented as mean $\pm S E, n=3$. Fluorescence intensity values have been normalized to the mean value of $(+)$. 


\subsection{Bioactive aggregation-induced emission systems}

Aggregation-induced emission (AIE) is a relatively unique property exhibited by some compounds that have the ability to show strong fluorescence only upon aggregation. Typical molecules that are prone to exhibit AIE have a highly dynamic, propeller-like structure bearing phenyl rings which are able to rotate against the central unit and annihilate excitons in solution. ${ }^{33-36}$ However, in the solid or in the aggregated state, this rotation is hindered leading to stacking of the molecules and with this $\pi$ - $\pi$-stacking charge transfer can occur leading to emission. This phenomenon has so far been investigated for diverse purposes such as organic light emitting diodes, ${ }^{37}$ sensors for metal ions ${ }^{38}$ and explosives, ${ }^{39}$ mapping of cell viscosity ${ }^{40}$ and cell targeting ${ }^{41}$ as well as interactions with bacteria ${ }^{42}$ and proteins. ${ }^{43}$ In particular, molecules that exhibit AIE in buffered media and can specifically interact with biological and medical samples are of great interest to biomedical and bioanalytical researchers since easily accessible fluorescent read-out is provided. Here we describe the use of bis(phenylthio)phthalonitrile (BPTP) derivatives, which show strong aggregationinduced emission in aqueous media, for selective aggregation and labelling of proteins and bacteria. The synthesized molecules have the ability to be easily equipped with bioactive compounds such as carbohydrates via $\mathrm{Cu}(\mathrm{I})$ catalysed click chemistry. In this study, we functionalized these molecules with bioactive a-D-mannose (1) and relatively non-fouling tetraethylene glycol (2) as shown in Figure 9.4a.

Dissolving these compounds either separately or as mixtures in water or buffer led to dispersed aggregates which exhibit strong fluorescence when excited at a wavelength $(\lambda)$ of $330 \mathrm{~nm}$. When the compounds were dissolved in THF or methanol only faint fluorescence was detected. Transmission electron microscopy of the negatively stained (1 wt\% uranyl acetate) compounds deposited from aqueous solutions on copper grids revealed round-shaped objects of ca. $16 \mathrm{~nm}$ in diameter as shown in Figure 9.4b. These results are indicative of aggregated species that are highly fluorescent in water whereas in a good solvent, such as THF, the aggregates are dispersed and nonfluorescent. The transition between fluorescent aggregates and non-fluorescent molecules was also visualized in a series of solutions of a varying ratio of THF and water with a constant concentration of (1) (100 $\mu \mathrm{M}$, Figure 9.4C). Interestingly, fluorescence emission seems to appear only beyond a water fraction of $80 \%$.

To test their applicability with biological systems, interaction studies between lectins and the BPTP-based aggregates were performed. To this end, mixtures of (1) and (2) in different ratios in buffer $(\mathrm{pH}=7.2,10 \mathrm{mM}$ HEPES, $137 \mathrm{mM} \mathrm{NaCl}, 1 \mathrm{mM} \mathrm{CaCl} 2,1 \mathrm{mM}$ 210 
$\mathrm{MnCl}_{2}$ ) were incubated with ConcanavalinA (ConA). As shown in Figure 9.4, increasing the amount of (1) in the aggregates induced agglutination, which did not occur when (1) was absent. This result shows that a specific interaction between the mannose moieties and the four binding pockets of the ConA occurs. The aggregation based on multivalent interactions between the mannose moieties and ConA occurs instantly upon addition of protein to the solutions containing BPTP-based aggregates and is strictly dependent on the amount of mannose in the aggregates. The agglutination starts with a concentration of around $25 \%$ of (1) in the aggregates composed of (1) and (2). When the competitor a-methyl-D-mannoside is added to the solution containing the precipitates of (1) and ConA, the precipitate can be re-dissolved indicating that the binding between ConA and mannose-functionalized aggregates is reversible (Figure 9.4e). When lectin peanut agglutinin (PNA) was used to interact with aggregates of (1), no agglutination was observed, confirming the specific binding of the mannose thiophthalonitrile to ConA.

In a final experiment the interaction between mannose-functionalized aggregates and bacteria was evaluated. To this end two strains of E. coli were tested. ORN208 bears mutated FimH receptors that should not be able to interact with the mannose BPTP derivatives. ORN178 expresses wild-type FimH receptors and should be able to recognize the mannose moieties in (1). ${ }^{44,45}$ Since each aggregate bears hundreds of mannose moieties multivalent recognition is expected and bacterial aggregation should occur. From Figure 9.4f it is apparent that ORN208 shows neither aggregation nor fluorescence on the bacteria upon incubation with $50 \mu \mathrm{M}$ (1) aggregates for $60 \mathrm{~min}$. In strong contrast, in the case of ORN 178, large bacterial aggregates with a size of around 60 to $100 \mu \mathrm{m}$ were observed. Fluorescence was found to be co-localized in the bacterial aggregates showing the presence of molecular BPTP aggregates between the single bacteria. Effective aggregation of bacteria was seen in less than $2 \mathrm{~h}$. Furthermore no toxicity of the BPTPs was observed towards the bacterial strains used in this study (data not shown). Even at higher concentrations no significant effect on the viability was observed. These experiments show that simple molecules like BPTP can develop extremely useful properties when they form supramolecular structures by aggregation and clustering through non-covalent interactions. Functionalization with bioactive ligands can further expand their applicability in the fields of diagnostics, imaging and biosensing. Studies are being conducted to gain further insights into the structure of the aggregates, their stability and spectral properties. Furthermore, investigations into the possibilities of engineering compounds that generate different fluorescence 
spectra, loading the aggregates with drugs and attaching different bioactive ligands are currently being pursued, highlighting the versatility of such systems.
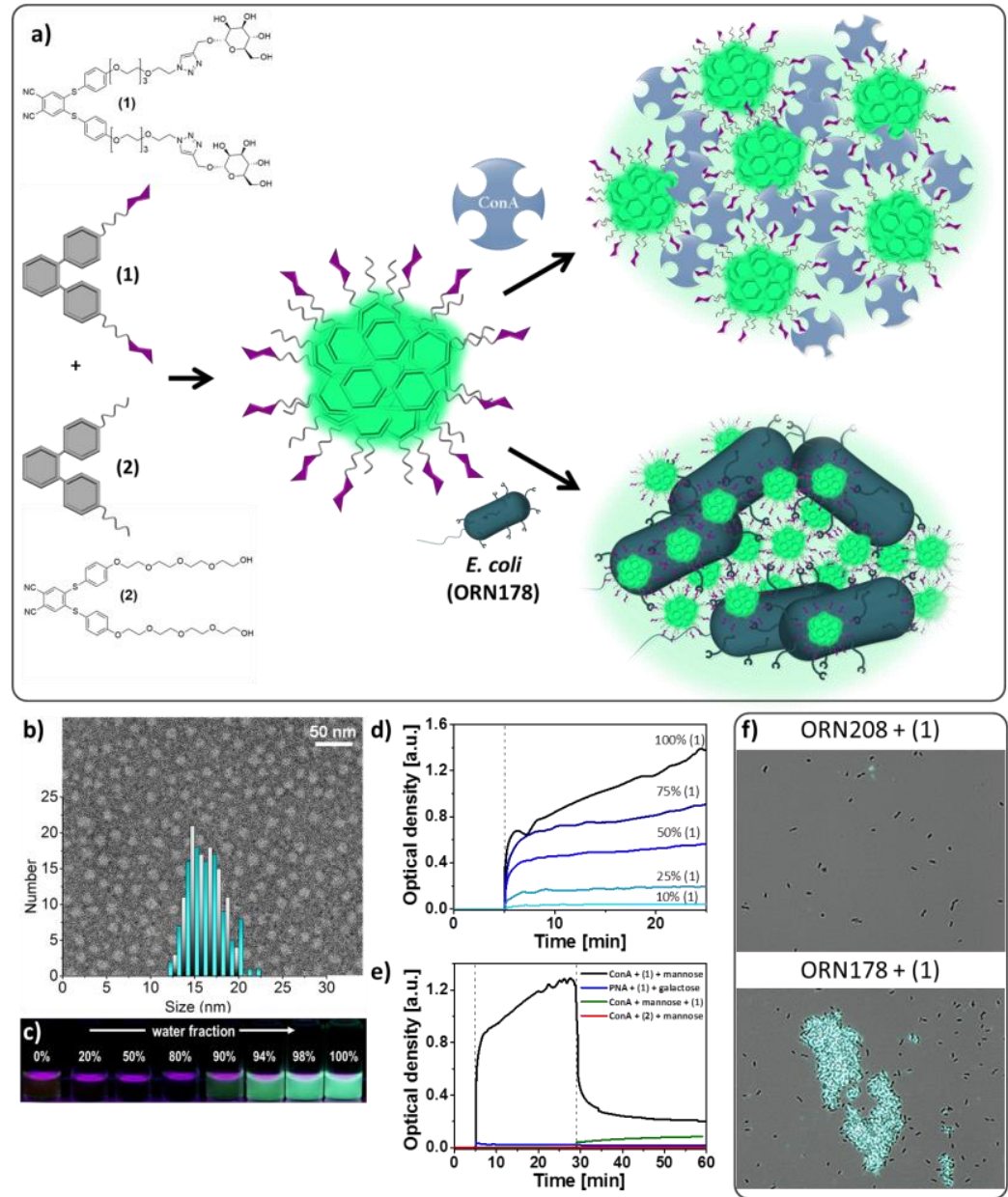

Figure 9.4. a) Structures of molecules used in this study and schematic presentation of the aggregation of ConA and E. coli in the presence of particles composed of (1) and (2). b) TEM image of $100 \mu \mathrm{M}$ aqueous solution of (1), negatively stained with $1 \mathrm{wt} \%$ uranyl acetate, and size distribution for TEM (blue-green) and SEM (black-grey) samples of (1). c) Photographs of $100 \mu M(1)$ in water-THF mixtures with increasing water fraction. d) Optical density measurement in dependency on time varying the ratio between (1) and (2), added to ConA; e) Optical density measurements in dependency on time. After 5 mins the thiophthalonitrile was added to the lectin solution and after 29 min the competitive sugar was added. f) Bright field and fluorescence microscopy overlaid images for bacterial aggregation induced by $50 \mu \mathrm{M}$ (1) in medium. Scale bar is $30 \mu \mathrm{m}$. 


\subsection{Acknowledgements}

We would like to thank the Laboratory of Biosensors and Bioelectronics, ETH Zurich for providing the facilities, assistance and training to perform the FluidFM based experiments. Mark Smithers and Dr. Rico Keim (University of Twente) are acknowledged for their help with SEM and TEM imaging.

\subsection{References}

(1) An, Q., Brinkmann, J., Huskens, J., Krabbenborg, S., de Boer, J., and Jonkheijm, P. (2012) A supramolecular system for the electrochemically controlled release of cells. Angew. Chem. Int. Ed. 51, 12233-12237.

(2) Brinkmann, Jenny, Sankaran, Shrikrishnan, Rinnen, Stefan, Arlinghaus, F. Heinrich, De Boer, Jan, and Jonkheijm Pascal. Achieving well defined reversible dynamic cell interfaces through supramolecular host-guest chemistry. Manuscript under preparation.

(3) Bush, M. E., Bouley, N. D., and Urbach, A. R. (2005) Charge-mediated recognition of Nterminal tryptophan in aqueous solution by a synthetic host. J. Am. Chem. Soc. 127, 14511-14517.

(4) Appel, E. A., Dyson, J., del Barrio, J., Walsh, Z., and Scherman, O. A. (2012) Formation of singlechain polymer nanoparticles in water through host-guest interactions. Angew. Chem. Int. Ed. 51, 4185-4189.

(5) Appel, E. A., Biedermann, F., Rauwald, U., Jones, S. T., Zayed, J. M., and Scherman, O. A. (2010) Supramolecular cross-linked networks via host-guest complexation with cucurbit[8]uril. J. Am. Chem. Soc. 132, 14251-14260.

(6) Massia, S. P., and Stark, J. (2001) Immobilized RGD peptides on surface-grafted dextran promote biospecific cell attachment. J. Biomed. Mater. Res. 56, 390-399.

(7) Auernheimer, J., Zukowski, D., Dahmen, C., Kantlehner, M., Enderle, A., Goodman, S. L., and Kessler, H. (2005) Titanium implant materials with improved biocompatibility through coating with phosphonate-anchored cyclic RGD peptides. ChemBioChem 6, 2034-2040.

(8) Patel, P. R., Kiser, R. C., Lu, Y. Y., Fong, E., Ho, W. C., Tirrell, D. A., and Grubbs, R. H. (2012) Synthesis and cell adhesive properties of linear and cyclic RGD functionalized polynorbornene thin films. Biomacromolecules 13, 2546-2553.

(9) Hersel, U., Dahmen, C., and Kessler, H. (2003) RGD modified polymers: biomaterials for stimulated cell adhesion and beyond. Synth. Biomim. Polym. 24, 4385-4415.

(10) Meister, A., Gabi, M., Behr, P., Studer, P., Vörös, J., Niedermann, P., Bitterli, J., Polesel-Maris, J., Liley, M., Heinzelmann, H., and Zambelli, T. (2009) FluidFM: combining atomic force microscopy and nanofluidics in a universal liquid delivery system for single cell applications and beyond. Nano Lett. 9, 2501-2507.

(11) Potthoff, E., Guillaume-Gentil, O., Ossola, D., Polesel-Maris, J., LeibundGut-Landmann, S., Zambelli, T., and Vorholt, J. A. (2012) Rapid and serial quantification of adhesion forces of yeast and mammalian cells. PLoS One 7, e52712.

(12) Putman, C. A., De Grooth, B. G., Van Hulst, N. F., and Greve, J. (1992) A detailed analysis of the optical beam deflection technique for use in atomic force microscopy. J. Appl. Phys. 72, 6-12.

(13) Sader, J. E., Chon, J. W., and Mulvaney, P. (1999) Calibration of rectangular atomic force microscope cantilevers. Rev. Sci. Instrum. 70, 3967-3969. 
(14) Gomez-Casado, A., Dam, H. H., Yilmaz, M. D., Florea, D., Jonkheijm, P., and Huskens, J. (2011) Probing multivalent interactions in a synthetic host-guest complex by dynamic force spectroscopy. J. Am. Chem. Soc. 133, 10849-10857.

(15) Evans, E., and Ritchie, K. (1997) Dynamic strength of molecular adhesion bonds. Biophys. J. 72, 1541-1555.

(16) Li, F., Redick, S. D., Erickson, H. P., and Moy, V. T. (2003) Force measurements of the $\alpha(5) \beta(1)$ integrin-fibronectin interaction. Biophys. J. 84, 1252-1262.

(17) Lehenkari, P. P., and Horton, M. A. (1999) Single integrin molecule adhesion forces in intact cells measured by atomic force microscopy. Biochem. Biophys. Res. Commun. 259, 645-650.

(18) Moore, S. W., Roca-Cusachs, P., and Sheetz, M. P. (2010) Stretchy proteins on stretchy substrates: the important elements of integrin-mediated rigidity sensing. Dev. Cell 19, 194-206.

(19) Liu, Y., Medda, R., Liu, Z., Galior, K., Yehl, K., Spatz, J. P., Cavalcanti-Adam, E. A., and Salaita, K. (2014) Nanoparticle tension probes patterned at the nanoscale: impact of integrin clustering on force transmission. Nano Lett. 14, 5539-5546.

(20) Young, M., Debbie, W., Uchida, M., and Douglas, T. (2008) Plant viruses as biotemplates for materials and their use in nanotechnology. Annu. Rev. Phytopathol. 46, 361-384.

(21) Ma, Y., Nolte, R. J. M., and Cornelissen, J. J. L. M. (2012) Virus-based nanocarriers for drug delivery. Approaches Drug Deliv. Based Princ. Supramol. Chem. 64, 811-825.

(22) Aljabali, A. A., Shukla, S., Lomonossoff, G. P., Steinmetz, N. F., and Evans, D. J. (2013) CPMVDOX delivers. Mol. Pharm. 10, 3-10.

(23) Brasch, M., de la Escosura, A., Ma, Y., Uetrecht, C., Heck, A. J. R., Torres, T., and Cornelissen, J. J. L. M. (2011) Encapsulation of phthalocyanine supramolecular stacks into virus-like particles. J. Am. Chem. Soc. 133, 6878-6881.

(24) Rurup, W. F., Verbij, F., Koay, M. S. T., Blum, C., Subramaniam, V., and Cornelissen, J. J. L. M. (2014) Predicting the loading of virus-like particles with fluorescent proteins. Biomacromolecules 15, 558-563.

(25) Resnick, A. (2012) Viral nanoparticles: tools for materials science and biomedicine, by Nicole Steinmetz and Marianne Manchester. Contemp. Phys. 53, 449-449.

(26) Medintz, I. L., Sapsford, K. E., Konnert, J. H., Chatterji, A., Lin, T., Johnson, J. E., and Mattoussi, H. (2005) Decoration of discretely immobilized cowpea mosaic virus with luminescent quantum dots. Langmuir 21, 5501-5510.

(27) Pokorski, J. K., and Steinmetz, N. F. (2011) The art of engineering viral nanoparticles. Mol. Pharm. 8, 29-43.

(28) Lee, L. A., Nguyen, H. G., and Wang, Q. (2011) Altering the landscape of viruses and bionanoparticles. Org. Biomol. Chem. 9, 6189-6195.

(29) Comellas-Aragones, M., Sikkema, F. D., Delaittre, G., Terry, A. E., King, S. M., Visser, D., Heenan, R. K., Nolte, R. J. M., Cornelissen, J. J. L. M., and Feiters, M. C. (2011) Solution scattering studies on a virus capsid protein as a building block for nanoscale assemblies. Soft Matter 7 , $11380-11391$.

(30) Hommersom, C. A., Matt, B., van der Ham, A., Cornelissen, J. J. L. M., and Katsonis, N. (2014) Versatile post-functionalization of the external shell of cowpea chlorotic mottle virus by using click chemistry. Org. Biomol. Chem. 12, 4065-4069.

(31) Tian, F., Jiao, D., Biedermann, F., and Scherman, O. A. (2012) Orthogonal switching of a single supramolecular complex. Nat Commun 3, 1207.

(32) Kumar, G. S., and Neckers, D. C. (1989) Photochemistry of azobenzene-containing polymers. Chem. Rev. 89, 1915-1925. 
(33) Hong, Y., Lam, J. W. Y., and Tang, B. Z. (2009) Aggregation-induced emission: phenomenon, mechanism and applications. Chem Commun 4332-4353.

(34) Chen, J., Xu, B., Ouyang, X., Tang, B. Z., and Cao, Y. (2004) Aggregation-induced emission of cis,cis-1,2,3,4-tetraphenylbutadiene from restricted intramolecular rotation. J Phys Chem A 108, $7522-7526$.

(35) Tong, H., Hong, Y., Dong, Y., Ren, Y., Häussler, M., Lam, J. W. Y., Wong, K. S., and Tang, B. Z. (2007) Color-tunable, aggregation-induced emission of a butterfly-shaped molecule comprising a pyran skeleton and two cholesteryl wings. J Phys Chem B 111, 2000-2007.

(36) Tong, H., Dong, Y., Hong, Y., Häussler, M., Lam, J. W. Y., Sung, H. H.-Y., Yu, X., Sun, J., Williams, I. D., Kwok, H. S., and Tang, B. Z. (2007) Aggregation-induced emission: effects of molecular structure, solid-state conformation, and morphological packing arrangement on lightemitting behaviors of diphenyldibenzofulvene derivatives. J Phys Chem C 111, 2287-2294.

(37) Huang, J., Tang, R., Zhang, T., Li, Q., Yu, G., Xie, S., Liu, Y., Ye, S., Qin, J., and Li, Z. (2014) A new approach to prepare efficient blue aie emitters for undoped OLEDs. Chem Eur J 20, 53175326.

(38) Zhang, L., Hu, W., Yu, L., and Wang, Y. (2015) Click synthesis of a novel triazole bridged AIE active cyclodextrin probe for specific detection of $\mathrm{Cd} 2+$. Chem Commun 51, 4298-4301.

(39) Qin, A., Lam, J. W. Y., Tang, L., Jim, C. K. W., Zhao, H., Sun, J., and Tang, B. Z. (2009)

Polytriazoles with aggregation-induced emission characteristics: synthesis by click polymerization and application as explosive chemosensors. Macromolecules 42, 1421-1424.

(40) Chen, S., Hong, Y., Zeng, Y., Sun, Q., Liu, Y., Zhao, E., Bai, G., Qu, J., Hao, J., and Tang, B. Z. (2015) Mapping live cell viscosity with an aggregation-induced emission fluorogen by means of two-photon fluorescence lifetime imaging. Chem Eur J 21, 4315-4320.

(41) Zhang, Y., Chen, Y., Li, X., Zhang, J., Chen, J., Xu, B., Fu, X., and Tian, W. (2014) Folic acidfunctionalized AIE Pdots based on amphiphilic PCL-b-PEG for targeted cell imaging. Polym Chem 5, 3824-3830.

(42) Müller, M., and Brunsveld, L. (2009) A supramolecular polymer as a self-assembling polyvalent scaffold. Angew Chem Int Ed 48, 2921-2924.

(43) Sanji, T., Shiraishi, K., and Tanaka, M. (2009) Sugar-phosphole oxide conjugates as "turn-on" luminescent sensors for lectins. ACS Appl Mater Interfaces 1, 270-273.

(44) Voskuhl, J., Sankaran, S., and Jonkheijm, P. (2014) Optical control over bioactive ligands at supramolecular surfaces. Chem. Commun. 50, 15144-15147.

(45) Harris, S. L., Spears, P. A., Havell, E. A., Hamrick, T. S., Horton, J. R., and Orndorff, P. E. (2001) Characterization of Escherichia colitype 1 pilus mutants with altered binding specificities. $J$. Bacteriol. 183, 4099-4102. 


\section{Summary}

For nearly over a decade, a wide variety of dynamic and responsive supramolecular architectures have been investigated and developed to address biological systems. Since the non-covalent interactions between individual molecular components in such architectures are similar to the interactions found in living systems, it was possible to integrate chemically-synthesized and naturally-occurring components to create platforms with interesting bioactive properties. Bacterial cells and recombinant proteins derived from them have been occasionally addressed and incorporated in such supramolecular biological systems, however a broader investigation into the various prospects of combining these two worlds has not yet been reported. This thesis represents an attempt to explore the possibilities of developing novel platforms by combining supramolecular chemistry with bacterial systems. The work described in this thesis has been organized in three sections -1 ) using bacteria to produce recombinant proteins that can interact with supramolecular hosts and target proteins in a multivalent and multi-specific manner, 2) developing supramolecular platforms that display bioactive ligands in a photo-responsive manner or as gradients to address bacteria as pathogens and 3) genetically engineering bacterial cells with a supramolecular binding motif in an attempt to incorporate them as living entities in supramolecular architectures. These studies provide a glimpse into some of the versatile ways in which supramolecular chemistry and bacterial systems can be combined. The components and concepts investigated in these chapters can be used interchangeably and also extended towards other fields to develop innumerable other systems. Some of these possibilities have been addressed in the epilogue where preliminary results from three projects involving mammalian cells, viral protein cages and aggregation-induced emission compounds have been briefly described. The general outlook gathered from these explorations indicates that a ripe future lies ahead for such supramolecular bacterial systems where the possibilities are limited only by imagination. 
218 


\section{Samenvatting}

Gedurende de jongste decennia zijn verschillende dynamische en responsieve supramoleculaire systemen onderzocht hoe ze zich verhouden als ze in aanraking komen met biologische systemen. Omdat niet-covalente interacties tussen moleculaire bouwstenen in supramoleculaire systemen sterk overeenkomen met de niet-covalente interacties in levende cellen, is er een ideale mogelijkheid geschapen om synthetische en levende componenten te integreren in een platform en te bestuderen hoe de bioactieve functionaliteit zich etableert. Bacteriële cellen en recombinante eiwitten zijn in de literatuur reeds op verschillende wijzen gecombineerd met supramoleculaire systemen, echter een bredere studie naar de mogelijkheden en beperkingen van de combinatie van deze twee werelden ontbreekt. Het werk in dit proefschrift laat uiteenlopende mogelijkheden zien van vernieuwende platformen waarbij supramoleculaire chemie is gecombineerd met bacteriële systemen. Hierbij is onderscheid gemaakt in 1) het gebruik van bacteriën voor de productie van recombinante eiwitten die kruisspecifieke interactie kunnen aangaan met andere eiwitten op meervoudige wijze, 2) het ontwikkelen van supramoleculaire platformen waarop bioactieve liganden lichtgevoelig of in een gradiënt vastgezet zijn en vervolgens gebruikt zijn voor bindingsstudies van bacteriën en pathogenen en 3) het genetisch introduceren van supramoleculaire bindingsplekken op transmembrane eiwitten in bacteriën in een poging om levende cellen op te nemen in supramoleculaire systemen. Gecombineerd laten deze studies zien dat de integratie van supramoleculaire chemie in bacteriële systemen veelzijdige nieuwe eigenschappen geeft. De bouwstenen en concepten die zijn onderzocht in dit proefschrift kunnen onderling uitgewisseld worden en uitgebreiding naar andere velden kan leiden tot ontelbare andere systemen. Een aanzet hiertoe is beschreven in een epiloog waarin de eerste resultaten van drie projecten zijn beschreven zoals het gebruik van dierlijke cellen, virale eiwit kooien en moleculen voor aggregatie-geïnduceerde emissie. Het onderzoek laat een veelbelovende toekomst zien voor supramoleculaire bacteriële systemen waarbij de mogelijkheden slechts gelimiteerd zijn door onze verbeelding. 


\section{Acknowledgements}

There are several people who have directly and indirectly contributed to making my doctoral studies a pleasant and enjoyable journey. I would like to take this opportunity to convey my deepest gratitude to all of you.

First, Pascal, I would like to thank you for giving me the opportunity to pursue this crucial phase of my education in your group. You have carefully populated and developed this multi-disciplinary group with friendly, open-minded, enthusiastic, collaborative and critical individuals, which has made working here a truly enjoyable experience. You have given me immense freedom and resources to carry out my research while still providing subtle guidance to keep me on a successful path. There are so many things l've learnt from you about managing science, life and people, which I hope to carry with me in my career.

Jurriaan, you were to be my promoter when I started my PhD and provided valuable insights in experimental design and analysis while I was learning the ropes in this field. So, I am very happy and honored that you agreed to be part of my PhD defense committee. Luc, Aldrik, Mireille and Séverine, I would also like to sincerely thank you for taking the time and effort of going through my thesis and, I am sure, contributing to a lively and constructive discussion during my defense.

Jeroen, you were involved in the projects performed by both the master students I supervised and always asked us the questions that set us thinking in the right direction. Nathalie, Tibor, Wim and Melissa, I always enjoyed our exchanges and would also like to thank you for maintaining an enjoyable place to do research. Marcel, Regine, Richard, Bianca, Nicole and Izabel you are the ones who keep this chaotic machinery of scientists running smoothly and have definitely enabled me to focus on research by handling all my technical and bureaucratic issues very effectively.

I would also like to convey my gratitude to Prof. V. Lakshminarayanan, my master thesis supervisor, for giving me the opportunity to explore the field of biophysical chemistry involving self-assembly, electrochemistry, surface chemistry, etc. and opening up the path that has led to this thesis.

Thanks to everyone from MNF and BNT, I have had a memorable time during my PhD both at work and outside. I have had a lot of fun times at the work weeks, conferences, parties, other gatherings and events for which I am grateful to several people. Raluca, 
Pieter, Jordi, Vijay, Melanie, Kim, Angel, you were around only during my first 2 years but thanks for making me feel very welcome at work and outside. Dodo, I am extremely grateful for the crash course you gave me on genetic and protein engineering at the very start of my PhD. I have always been amazed that you seem to have experience in almost every laboratory technique used around here and am glad that I could always count on you for advice when I was having trouble. Rajesh and Tushar, thanks a million for being with me from the first day I landed in Enschede and making sure I settled in very comfortably. Rajesh, I was truly honored to be your paranymph and enjoyed it a lot. Carmen, thank you for patiently answering all my questions in the realm of chemistry, no matter how silly they may have been. Rik, I truly appreciate the way you had set up the ML1 lab and prepared protocols for several biochemical characterization techniques, allowing me to rapidly perform them. Ivan, thanks for being an extremely entertaining collaborator who made those long and arduous experiments so much fun. Mustafa, you were an excellent summer student twice and worked with undaunted enthusiasm on unexpectedly complex projects. Thanks to you I saved months of work in optimizing the genetic and protein engineering protocols. Mark and Nora, I was extremely lucky to be able to supervise your Master projects. I personally learnt a great deal from all the experiments you performed with great perseverance. Albert Heck, thank you for the collaboration to detect our protein complexes via mass spec and Arjan, thanks a million for patiently performing the tedious measurements and providing extremely detailed analyses.

Jasper, my super fun and efficient collaborator and paranymph, Thomas, my calm and calculating badminton partner and paranymph, Raquel, the CrAZieSt and warmest person I know, Emanuela, super kind and hyper social, Mark, my "Magnetism or some such" buddy, Roberto, the multi-talented La Chimba, Marina, sweet and ever smiling, Carlo, the suave and stylish Italian, Mariska, the bold and the beautiful, Jens, the super synthesizer and efficient collaborator, Diana and Ireneus, the sporty and adventurous couple, Betty, the fun-loving Frisbee girl and fellow 9GAGGER, Maike, the horse-loving cake factory girl, Alejandro, the smart and meticulous chemist, Sarah, the incarnation of dance and fellow comic lover, Laura, my sporty and cheerful car-share buddy, Gulistan, the gentle and thorough scientist, Janneke, my brilliant neighbor and Bread-Friday girl, Ben, my PlayStation pal, Andrea, the smart and happy chemist, Nana, the party-hard modelling girl, Francesco and Veronica, the incredibly fun and wacky couple: thank you all for inviting me to your homes and parties, helping me move, accompanying me on various trips, joining me for movies and contributing to my happiness by being like a family to me. I'm also extremely grateful for being able to work with fun, pleasant and 
joyful colleagues like Rick, Stan, Sven, Yunglong, Wouter, Liang, Christoph, Alexis, Wilfred, He, Tetiana, Pauline, Weis, Supitch Shuqin, Rianne, Robin, Supa, Frederico, Aijie, Rindia, Daniele, Robin, Nicole, Liulin, Alexander, Jacopo, João, Tom, Timon, Amal, Shyam.

Janos and Tomaso, thanks for making me feel welcome and allowing me to work at LBB with the FluidFM system. It was a very educational experience both scientifically and personally. Leena, thanks for being an extremely thoughtful, friendly, fun, innovative and sincere collaborator. Benji, thanks for your detailed description of the work done in the group, which made it possible to find an interesting project to do there. Mathias, László, Harald, Livie, Andreas, Volker, Marco, Hana, Luca, Stephanie, Vincent, Esther, Flurin, Alex, Greta, Raphael, Klas, Serge, Csaba, Victoria and everyone in LBB, thank you all for the wonderful time I had there and hope to be back soon. I would also like to dearly thank all the BeeZ who made Zürich feel like another home for me.

Most of all I would like to thank my family for their immeasurable love, support and encouragement in all my endeavors, both personally and professionally. Appa, Amma, Shyam, Paati, Thatha and Ammamma, you have all been a constant source of stability in my life and have given me the confidence to take risks and explore various possibilities available to me. Doris Mami, thank you for your whole-hearted pride and confidence in me, which is something I constantly strive to live up to.

Finally, I would like to thank Jenny, my wonderful wife, friend, colleague and collaborator. We started together on the same day as co-workers and have kept growing closer ever since. You have constantly contributed to my scientific, social and personal successes that have made this thesis a reality. I cannot sufficiently express my appreciation for all you have done for me with mere words.

There have been so many people who have contributed to this thesis and me as a scientist in so many ways that these pages are insufficient to express my complete gratitude. For those reading this, please know that my gratitude extends far beyond what has been listed here. 


\section{About the Author}

Shrikrishnan Sankaran was born in Bangalore, India on the $7^{\text {th }}$ of January 1988. Willing to learn the basics of bioinstrumentation, he pursued a 5 year sandwich program, from 2006 - 2011, to obtain a Bachelor of Engineering (Hons) degree in Electronics and Instrumentation Engineering and a Master of Science (Hons) degree in Biological Science from the Birla Institute of Technology and Science - Pilani (BITS-Pilani). In the summer after the $2^{\text {nd }}$ year of this program, he worked as an intern at the Medical Research Foundataion of the Sankara Nethralaya Eye Hospital, Chennai, India with Dr. J. Biswas and Dr. S. Sridharan. He was involved in the first retrospective study of ocular lesions in 1,000 consecutive HIV-positive patients in India. During the summer of the $3^{\text {rd }}$ year, he learnt vital skills in genetic and protein engineering, working on a summer project dealing with the site directed mutagenesis of EGFP altering its fluorescence properties, at the Center for Biotechnology, Anna University, Chennai, India with Prof. K. Sankaran and Dr. S. Hamsanathan. During the $5^{\text {th }}$ year, he did a joint dissertation dealing with electrochemical detection in biological samples with Prof. V. Lakshminarayanan in the Soft Condensed Matter Laboratory of the Raman Research Institute in Bangalore, India. During this period, he performed two major projects - The first involved detection of protein adsorption to charged SAMs on gold electrodes using electrochemical impedance measurements and the second involved studying the electron transfer properties of redox probes in bovine milk for the development of analytical sensors in the dairy industry.

Encouraged by these enjoyable experiences in research, he has been working on developing Supramolecular Bacterial Systems as part of his doctoral studies, since October 2011, with Prof. Dr. ir. P. Jonkheijm in the 'Bioinspired Molecular Engineering Laboratory' of the MIRA Institute for Biomedical Technology and Technical Medicine and the 'Molecular Nanofabrication Group' of the MESA+ Institute for Nanotechnology at the University of Twente, Enschede, The Netherlands. In 2015 he did a 2 month collaborative project in ETH Zürich, Switzerland with Prof. J. Vörös, Dr. T. Zambelli and L. Jaatinen studying adhesion forces of mammalian cells on supramolecular surfaces using FluidFM. The results of his research work during the $\mathrm{PhD}$ have been presented in this thesis. 


\section{List of Publications}

\section{Published}

1. S. Sankaran,, J. van Weerd, ${ }^{\#}$ J. Voskuhl, M. Karperien and P. Jonkheijm "Photo-responsive cucurbit[8]uril-mediated adhesion of bacteria on supported lipid bilayers"

Small, 2015, doi: 10.1002/smll.201502471

2. S. Sankaran, ${ }^{\#}$ M. de Ruiter, ${ }^{\#}$ J.J.L.M. Cornelissen and P. Jonkheijm

"Supramolecular surface immobilization of knottin derivatives for dynamic display of high affinity binders"

Bioconj. Chem., 2015, 26 (9), 1972-1980

3. S. Sankaran, M. C. Kiren, and P. Jonkheijm

"Incorporating Bacteria as a Living Component in Supramolecular Self-Assembled Monolayers through Dynamic Nanoscale Interactions"

ACS Nano, 2015, 9(4), 3579-3586.

4. J. Voskuhl, ${ }^{\#}$ S. Sankaran ${ }^{\#}$ and P. Jonkheijm

"Optical control over bioactive ligands at supramolecular surfaces"

Chem. Commun., 2014, 50(96), 15144-15147. (Back cover)

5. J. Brinkmann, ${ }^{\#}$ E. Cavatorta, ${ }^{\#}$ B. Schmidt, ${ }^{\#}$ S. Sankaran, ${ }^{\#}$ J. van Weerd, ${ }^{\#}$ and P. Jonkheijm

"About supramolecular systems for dynamically probing cells"

Chem. Soc. Rev., 2014, 43(13), 4449-4469. (Inside back cover)

6. S. Sankaran, K. Sankaran, and V. Lakshminarayanan

"Electrochemical Impedance Analysis of Adsorption and Enzyme Kinetics of Calf Intestine Alkaline Phosphatase on SAM-Modified Gold Electrode"

J. Phys. Chem. C, 2012, 116(30), 16030-16037.

7. S. Sankaran and V. Lakshminarayanan

"Electron transfer studies of redox probes in bovine milk"

J. Colloid Interf. Sci., vol. 370, issue 1, 2012, pp. 124-131. 
8. S. Sudharshan, S. Kaleemunnisha, A. Banu, S. Sankaran, A. George, B. R. Babu, B. Devaleenal, N. Kumarasamy, and J. Biswas

"Ocular lesions in 1,000 consecutive HIV-positive patients in India: a long-term study"

J. Ophthal. Inflamm. Infect., 2013, 3(1), 1-7.

\section{Submitted}

9. J. van Weerd, ${ }^{\#}$ S. Sankaran, ${ }^{\#}$ O. Roling, S. Sukas, B-J. Ravoo, S. O. Krabbenborg, J. Huskens, S. le Gac, M. Karperien and P. Jonkheijm

"Probing the threshold in bacteria-mannose recognition using locked-in supported lipid bilayer gradients"

Submitted to Adv. Func. Mat., 2015.

10. J. Brinkmann, S. Sankaran, S. Rinnen, H. F. Arlinghaus, J. de Boer and P. Jonkheijm "Cellular Response to Dynamic Cell Bio-interfaces Employing CB[8]-Mediated RGD Ligand Assembly"

Submitted to J. Am. Chem. Soc., 2015.

11. J. Voskuhl, ${ }^{\#}$ B. Schmidt, ${ }^{\#}$ S. Sankaran, L. Stegemann, C. Strassert, J. Ak, M. Waller, P. Jonkheijm

"Mannose-functionalized fluorescent aggregates of Thiophthalonitriles interact with bacteria"

Submitted to Chem. Commun., 2015.

12. C.H. Gayathri, S. Sankaran, J. H. Shah and K. Sankaran

"Ultrasensitive and Selective Supramolecular Immuno Nanoparticle (SIN) Based Bacterial Pathogen Detection"

Submitted to Colloids Surf., B, 2015.

\section{In Preparation}

13. N.L. Weineisen, S. Sankaran,, ${ }^{\#}$ C.A. Hommersom, ${ }^{\#}$ J. Voskuhl, ${ }^{\#}$ N. Katsonis, P. Jonkheijm and J.J.L.M. Cornelissen

"Photo-responsive, reversible immobilization of virus particles on supramolecular platform" 
14. S. Sankaran, I. Stojanovic, A. Barendregt, A.J.R. Heck, R.B.M. Schasfoort and P. Jonkheijm*

"Scaffolding of cystine-stabilized miniproteins"

15. S. Sankaran, ${ }^{\#}$ L. Jaatinen, ${ }^{\#}$ J. Brinkmann, T. Zambelli, J. Vörös and P.Jonkheijm "Studying cellular adhesion forces on supramolecular self-assembled monolayers using FluidFM"

16. Y. Yu, S. Sankaran, P. Jonkheijm, S. de Beer, J. Vancso

"Switchable friction and adhesion triggered by light" 


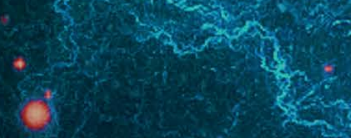

d

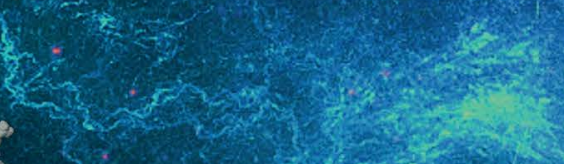
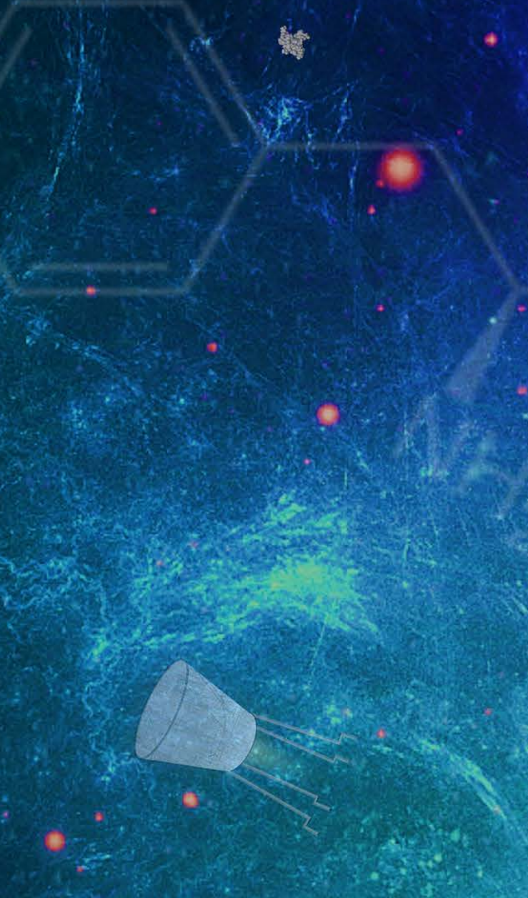

4.
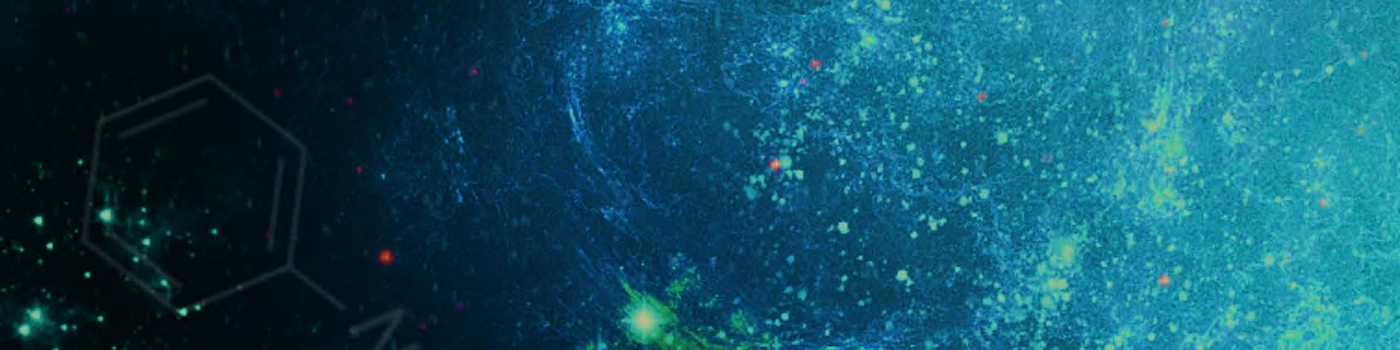

d.

(1) $)^{2}$

2

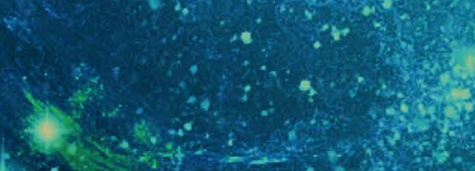

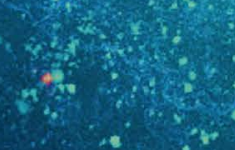

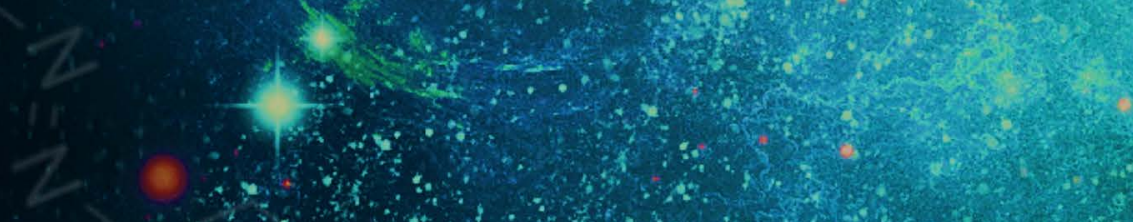

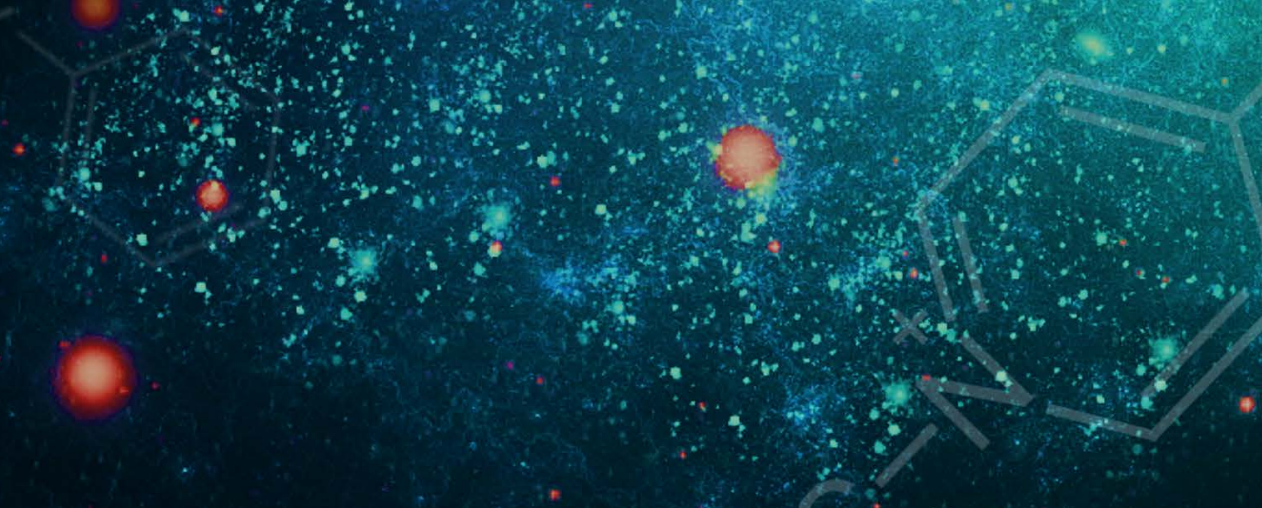

